\title{
41st Annual Meeting of the International Society for Pediatric Neurosurgery, Mainz, Germany, Sept 29 Oct 3, 2013
}

This supplement was not sponsored by outside commercial interests. It was funded entirely by the publisher.

\author{
Kyu-Chang Wang, ISPN President \\ Wolfgang Wagner, ISPN Meeting Chair \\ Young-Shin Ra, ISPN Scientific Chair
}

\section{Oral presentations}

\section{Adult Outcome of CNS malformations}

\section{1.}

Pregnancy of patients with myelomeningocele

Mami Yamasaki ${ }^{1}$, M. Yoshida ${ }^{2}$, Takumi Yamanaka ${ }^{1}$, Atsuko Harada ${ }^{1}$, Masahiro Nonaka ${ }^{3}, \mathrm{~S}$. Momose ${ }^{4}$

${ }^{1}$ Department of Pediatric neurosurgery, Takatsuki General Hospital(TGH), Takatsuki, Osaka; ${ }^{2}$ Department of obstetrics and gynecology, Hoshigaoka Kosei-Nenkin Hospital (HKH), Hirakata, Osaka; ${ }^{3}$ Department of Neurosurgery, Osaka National Hospital (ONH), National Hospital Organization, Osaka. City, Osaka; ${ }^{4}$ Department of urology, Hoshigaoka Kosei-Nenkin Hospital, Hirakata, Osaka, Japan

Aim: Many patients with myelomeningocele (MMC) have reached reproductive age; therefore we focused on the pregnancy of the patients with MMC. The aim of this paper is to clarify the problems of neurosurgical management, especially hydrocephalus $(\mathrm{HC})$ during pregnancy and genetic counseling before pregnancy of patients with myelomeningocele. Materials \& Methods: In these 3 institutes, 6 pregnancies in 5 patients with $\mathrm{MMC}+\mathrm{HC}$ and 12 pregnancies in 9 patients with $\mathrm{MMC}$ - $\mathrm{HC}$ were experienced. In this paper, we made retrospective evaluation focusing on pregnancy with $\mathrm{MMC}+\mathrm{HC}$ and genetic counseling.

Results: 1. In $\mathrm{MMC}+\mathrm{HC}$ group, 3 cases were delivered by caesarian sections (CS), 3 cases were by trans virginal delivery (VD), in MMC-HC group, $\mathrm{CS}$ were performed in 4 cases, VD in 6 cases. In other 2 cases, one case showed fetal death due to maternal hypertension and the other terminated pregnancy due to fetal abnormality. In all cases of both groups, $\mathrm{CS}$ was performed due to obstetrics indication. 2. External drainage was performed in one case to prevent shunt infection due to amniotic turbidity after delivery. No cases required intensive externalization of shunt. 3. One case had family history with MMC. In two cases, fetuses showed abnormality. One case showed multiple malformation (abdominal and chest wall defect, deformity of extremities) and the other showed $\mathrm{MMC}+\mathrm{HC}$.

Conclusion: These patients require careful urological, obstetric and neurological care. Molecular genetic study in spinal dysraphism is needed for genetic counseling.
2

Long-term functional \& cognitive outcome in Spina Bifida-Chiari II malformation - A cross-sectional survey of adult patients

Jonathan R. Ellenbogen ${ }^{1}$, Chris J. Parks ${ }^{1}$, Michael D. Jenkinson ${ }^{2}$, Ann Flynn $^{3}$, Peter Murphy ${ }^{3}$, Conor L. Mallucci ${ }^{1}$

${ }^{1}$ Department of Neurosurgery, Alder Hey Children's NHS Foundation Trust, Liverpool; ${ }^{2}$ Department of Neurosurgery, The Walton Centre NHS Foundation Trust, Liverpool; ${ }^{3}$ Department of Neuropsychology, The Walton Centre NHS Foundation Trust, Liverpool, UK

Introduction: Open spina bifida is frequently associated with Chiari II malformation and hydrocephalus. The long-term outcomes in adults with these conditions are not fully appreciated. Neuropsychological testing is increasingly important in terms of patient reported outcome measures. We investigated the disability, lifestyle, social-emotional and cognitive function in adults who had myelomeningocoele closure at birth.

Methods: Cross-sectional survey of adult patients with Spina Bifida-Chiari II Malformation. Patients completed lifestyle and hydrocephalus outcome questionnaires (HOQ) and underwent cognitive testing. Clinical variables which may influence outcome including number of shunt revisions, shunt infection and surgical decompression of foramen magnum, were investigated.

Results: Twenty-one adults, with a median age of 35 years, were investigated. All had CSF diversion for hydrocephalus, with the majority (81\%) performed before the age of 6 months. Eight patients had foramen magnum decompression for headache or progressive brainstem symptoms with stabilisation of symptoms in seven and improvement in one. Eight patients (38\%) were living independently, 33\% were ambulant unassisted or with aids, $52 \%$ drove a car and only $24 \%$ were in paid employment. HOQ scores for cognitive function were lower $(0.56 \pm 0.20$; mean \pm standard deviation (SD)) than those for physical $(0.64 \pm 0.15)$ and social-emotional $(0.65 \pm$ $0.17)$ health, with attention and memory most severely affected. Repeated episodes of shunt malfunction or foramen magnum decompression were not associated with a worse cognitive function.

Conclusions: In spite of early intervention in infancy patients with Spina Bifida-Chiari II Malformation have significant cognitive impairment with a poor prognosis for independent living.

\section{3}

Long-term follow-up in open spina bifida: transition from childhood to adulthood

Dieter Class, Henriette Wolko, Raimund Firsching

Department of Neurosurgery, Otto von Guericke University, Magdeburg, Germany

Introduction: More than $90 \%$ of the children born in the western world with open spina bifida have a prolonged life expectancy and meanwhile 
grow into adulthood due to improved medical care. Many questions referring to specific problems of adults including fatal risk factors are open. The fate of many patients is unclear. We made a comprehensive inquiry to evaluate specific problems in this age group.

Methods: An inquiry form including 34 questions was sent to the own patient group, to all neurosurgical departments in Germany and distributed via the Spina bifida Association in Germany $(\mathrm{ASbH})$. The questionnaire was also available in the internet. Questions referred to details of the disease, treatment, social care and also aspects of life satisfaction. Results were evaluated anonymously.

Results: The mean age was 35,5 years. Mainly women are affected (64\%). About $85 \%$ patients needed surgery for hydrocephalus but only exceptionally surgery for Chiari malformation and tethered cord was done. Mean number of hospital admissions was 9. Other operations were necessary due to urological problems and for correction of limb deformities. $60 \%$ of the patients are working regularly, about $75 \%$ declare they are basically satisfied with their situation. There was one adult with unexpected death.

Conclusion: The current standard of treatment in childhood resulted in longer survival. Continuous follow-up and data collection is mandatory also to detect possibly fatal risks early and to provide care for the adult patients when their parents are not in a position to help any more.

Operated Closed Spinal Dysraphism (CSD): Transition to adulthood Laura Grazia Valentini ${ }^{1}$, Giorgio Selvaggio ${ }^{2}$, Alessandra Erbetta ${ }^{3}$, Roberto Cordella ${ }^{1}$, MariaGiovanna Pecoraro ${ }^{4}$, Stefania Bova ${ }^{5}$, E. Boni ${ }^{2}$, Elena Beretta $^{1}$, Marika Furlanetto ${ }^{1}$

${ }^{1}$ Neurosurgery Department, ${ }^{3}$ Neuroradiology Service, Fondazione Istituto Neurologico "Carlo Besta" Milan; ${ }^{2}$ Pediatric Surgery Unit, ${ }^{5}$ Child Neurology Unit, ICP, Children Hospital "Vittore Buzzi", Milan, Italy; ${ }^{4}$ Neuroradiology Unit, Ospedale Bolognini-Seriate, Bergamo, Italy

Closed Spina Bifida causes progressive neurological deterioration due to "tethering"; prophylactic surgery is widely accepted for filar lipomas, still debated for the Conus Lipomas and Complex terthering. We reviewed 116 operated children, retrospectively reclassified following Pang's criteria; intraoperative NeuroPhysiological Monitoring (NPM) was applied since 1998; 9 cases were associated to Anorectal (ARM) and 9 to Chiari (CM1) Malformation. $75 \%$ were symptomatic at surgery, 44\% experiencing progressive preoperative deterioration. Post-operative surgery-related deterioration was $6 \%$, reduced by NPM and increased by reoperations. Surgery failed improving definitive deficits, especially of sphincter functions; the urodynamic (UD) exams were the most sensitive test in preoperative to discover early preoperative worsening, prone to postoperative improvement restoring normal function, and in follow-up (FUP) to define delayed deterioration deserving re-do surgery. Lengthening FUP showed increasing percentage of re-tethering in complex cases submitted to partial surgery; 4/36 children evaluated longer than 8 years needed reoperation. These results influenced surgical indication and technique: we progressively started to operate asymptomatic children to maintain sphincter function by a more radical operation to prevent retethering. The analysis demonstrated that deterioration of a conservative approach may be worse than the results of surgery, performed by a team with special expertise. First surgery should be aimed to complete detethering, by support of NPM, because second surgery carries higher risks. UD testing and MRI in prone position were the best tools for screening children at risk of retethering with growth, eventually deserving reoperation; they are benefits of a multidisci plinary approach.
Long-term outcome of congenital hydrocephalus therapy Matthias Preuß ${ }^{1}$, Ulf Nestler ${ }^{1}$, M. Weißer ${ }^{2}$, Christian Geyer ${ }^{2}$

${ }^{1}$ Department of Neurosurgery, ${ }^{2}$ Department of Pediatric Surgery, University Hospital Leipzig, Germany

In order to evaluate the longterm outcome of congenital hydrocephalus 65 patients diagnosed with congenital hydrocephalus treated within the first 12 months of life between 1971 and 1987 at the University Hospital Leipzig, Germany, have been reviewed. Mean age of the patients at last follow-up was 32 years (range 25-41 years). Results: 6 patients $(9.2 \%)$ became shunt-independent during adulthood. $21.7 \%$ of the shunt valves did survive without revision (up to 35 years). By the age of $20,81.0 \%$ of the patients have required at least one shunt revision, by the age of $30100 \%$ of the patients. Shunt infections occurred in $5.1 \%$ of the primary insertions ( 3 patients) and within the first year after implantation. Two patients have been converted to etV successfully. When shunt failure occurred, $84.6 \%$ presented with typical symptoms of raised intracranial pressure. 9.2\% experienced rapid deterioration of vision as only symptom of late shunt dysfunction in adulthood. Only one of nine patients regained functional vision despite prompt surgical revision. Because of the retrospective analysis, mortality rate cannot be quantified. From 57 evaluated patients, $45.6 \%(\mathrm{n}=26)$ had a good outcome (modified Rankin Scale $m R S=0 / 1)$, no impairment was found in $24.6 \%(n=14)$. Motor deficits have been found $45.6 \%$, no functional vision has been detected in $14.3 \%$ of the patients. Visual impairment has been found in $29(59.2 \%)$ and hearing impairment in 2 patients $(2.2 \%)$. Epilepsy incidence was $43.9 \%$. From 53 evaluated patients, 37.7\% $(\mathrm{n}=20)$ attended regular schooling, $54.8 \quad(\mathrm{n}=29)$ attended school for handicapped students and $7.5 \%(\mathrm{n}=4)$ were not able to attend school. One patient finished university studies $(1.8 \%)$. 22 patients $(41.5 \%)$ work in a sheltered working environment. In summary, 35 patients work on a daily basis $(66.6 \%)$.

\section{6}

Shunt-depending patients with infantile hydrocephalus - today on age over 20 years

Jivko K. Surchev ${ }^{1}$, Ludmila P. Todorova ${ }^{2}$, Kiril D. Georgiev ${ }^{1}$, Asen A. Hadjzhiyanev $^{1}$, Delyan V. Ferdinandov ${ }^{1}$

${ }^{1}$ Department of Neurosurgery, Medical University, ${ }^{2}$ Institute of Biophysics and Biomedical Engineering, Bulgarian Academy of Sciences

Sofia, Bulgaria

Introduction: We analyzed what has happened with patients hunted in childhood, when they are on age over 20 years today.

Material and methods: For the 20-years period in the Department of Neurosurgery, Medical University Sofia, 242 children with infantile hydrocephalus were shunted. As of today, 79 of them (33 females, 46 male) were shunted in the interval between 1984 and 1999 and now they are on age over 20 years (females on age to 37 years, males - to 40 years). Results: In the group of females $49 \%$ are with " 3 and more revisions" while in the group of males they are $35 \%$. In the females the mean interval to the first revision is 5,5 years and in males it is 4,6 years. We made frequency distribution by sex of shunt survival after the last revision until 2013. We calculated the mathematical index K for shunt survival in both groups. In our study the mean age of the females is 27 years. We found out that 5 women give birth -3 of them to one child and 2 of them - to two children. All of them were with a normal pregnancy.

Conclusions: The results from comparing the indexes for both groups males and females, in long-term period, are contradictory: in females the mean interval to the first revision is longer, but also the percentage of them with " 3 and more revisions" is higher. The mathematical index $\mathrm{K}$ is 
higher among the females. The mean survival period in the whole contingent after last revision until 2013 is 14,7 years. This inspires optimism for this "grown up children".

\section{Adolescence and adulthood in former premature infants} with posthemorrhagic hydrocephalus

Andrea Reinprecht, Klaus Novak, Christian Dorfer, Thomas Czech University of Vienna Medical School, Department of Neurosurgery, Vienna, Austria

In the management of posthemorrhagic hydrocephalus in premature infants, a main concern of parents and doctors involved in treatment is the question of long-term prognosis and quality of life. We performed a long-term follow-up in a series of 42 initially premature infants shunted for posthemorrhagic hydrocephalus. Patients mean gestational age was 29.8 weeks, mean birthweight 1363.8 g. $92.8 \%$ had intraventricular hemorrhage grade III or IV (Volpe classification). Hydrocephalus was initially treated by external ventricular drainage and by a definite shunt at the mean age of 58.9 days. At follow-up, patients were 29 to 14 years old (mean 20,75 ys). Three patients died, 12 patients were lost to follow-up $(28,6 \%)$. Half of the patients were without or with mild deficit. Severe disability appeared in 3 patients after IVH grade IV and in all patients with additional periventricular leukomalacia. Neurologic outcome was not correlated to number of EVDs or number of shunt revisions, to gestational age or birth weight. Due to the small collective, correlation to grade of IVH did not reach significance.

Forty years outcome in 128 hydrocephalic patients shunted during childhood

Anne H. Paulsen, Tryggve Lundar, Karl-Fredrik Lindegaard

Department of Neurosurgery, Oslo University Hospital, Rikshospitalet, Oslo, Norway

Introduction: The goals were to determine 40-year outcome in adults with childhood hydrocephalus and to assess their health-related quality of life.

Methods: Patients younger than 15 years of age, first-time shunt treated for hydrocephalus in the calendar years 1967-1970, were included in a retrospective study on surgical morbidity, mortality rates, academic achievement, work participation, perceived health and functional status.

Results: Overall, 128 patients were included, no patient being lost to follow-up. For the 40-year period, the overall mortality rate was $47.5 \%$ (61/128). After two years, 97 patients $(76 \%)$ were still alive. The etiological composition of the cohort; MMC 33\%, neoplasms $20 \%$, congenital communicating HC $16 \%$, idiopathic $8 \%$, IVH $7 \%$, aqueduct stenosis $5 \%$, intracranial cysts $5 \%$, postmeningitis $\mathrm{HC}$ $3 \%$ and others $3 \%$. Shunt revisions were performed in 422 instances. Mean annual revision rate during first, second, third, and forth decade after primary shunt insertion were $0.29,0.05,0.04$, and 0.05 respectively. 27 patients $(21 \%)$ have had no revision of the shunt, 5 patients $(3.9 \%)$ of whom are still alive at follow-up. 33 patients $(25.8 \%)$ are living with a shunt system which has not been revised in thirty years or more. Compared with the reference group the hydrocephalic cohort reported a poorer perceived health in 6 of 8 SF-36 domains. At follow-up, 21/67 (31\%) were full or partly employed.

Conclusion: During the 40-year follow-up period the mortality rate was high. Shunt-related mortality rate was $5.5 \%$. Shunt placement to treat childhood hydrocephalus has a substantial effect on social functioning and perceived health in middle-aged adult patients, but is less disheartening than expected.

\section{Raimondi Award Presentation}

9

Pathoembryogenesis of terminal myelocystocele: Terminal balloon in secondary neurulation of the chick embryo

Ji Yeoun Lee ${ }^{1}$, Saet Pyoul Kim ${ }^{1}$, Shin Won Kim ${ }^{1}$, Sung-Hye Park ${ }^{2}$, Jung Won Choi ${ }^{1}$, Ji Hoon $\mathrm{Phi}^{1}$, Seung-Ki Kim ${ }^{1}$, Dachling Pang ${ }^{3,4}$, Kyu-Chang Wang ${ }^{1}$

${ }^{1}$ Division of Pediatric Neurosurgery, Children's Hospital, Seoul National University, College of Medicine; ${ }^{2}$ Department of Pathology, Seoul National University Hospital, College of Medicine, Seoul, Korea; ${ }^{3}$ Department of Pediatric Neurosurgery, University of California, Davis USA; ${ }^{4}$ Regional Center of Pediatric Neurosurgery, Oakland Medical Center, Kaiser Foundation Hospitals of Northern California,

Background: Terminal myelocystocele (TMC) is thought to be caused by a misstep during secondary neurulation. However, due to the paucity of data on secondary neurulation and the rarity of TMC, proofs of this pathogenetic mechanism are unavailable. Objective Based on a previous observation that TMC resembles a step of secondary neurulation in chick, a closer look was taken at secondary neurulation of chick embryos focusing on the cerebrospinal fluid-filled distal neural tube (terminal balloon)

Methods: Chick embryos at Hamburger and Hamilton $(\mathrm{H}-\mathrm{H})$ stages of 28 , $30,33,35,37$, and 40 were harvested. Hematoxylin-eosin staining, additional immunohistochemistry (laminin, cytokeratin, nestin), and scanning electron microscopy were performed.

Results: In H-H stages 28 to 30, after merging of the lumina of the primary and secondary neural tubes, the caudal end of the confluent tube dilates into a balloon-like structure (terminal balloon). As the proximal tube progressively becomes narrower, the terminal balloon dilates even further and its wall fuses with the surface ectoderm $(\mathrm{H}-\mathrm{H}$ stage 33). Later in $\mathrm{H}-\mathrm{H}$ stages 35 to 40 , the terminal balloon shrinks and becomes detached from the surface ectoderm and ultimately disappears as the proximal lumen of the secondary neural tube continues to collapse.

Conclusion: A dilated balloon doubtlessly exists in the terminal secondary neural tube in chick embryos, and its subsequent disappearance occurs in a variable time course and sequence. Arrest of apoptosis resulting in failure of detachment of the terminal balloon from the surface ectoderm may well be the basis for human TMC.

\section{Dysraphism and Tethered Spinal Cord}

\section{0}

Defining a neurosurgical algorithm for open fetal myelomeningocele closure

Robert J. Bollo $^{1,2}$, William E. Whitehead ${ }^{1,2}$, Darrell L. Cass ${ }^{2,3,4}$, Oluyinka O. Olutoye ${ }^{2,3,4}$, Michael A. Belfort ${ }^{2,4}$, Rodrigo Ruano ${ }^{2,4}$, Andrew Jea ${ }^{1,2}$

${ }^{1}$ Division of Pediatric Neurosurgery, Texas Children's Hospital, Department of Neurosurgery, Baylor College of Medicine; ${ }^{2}$ Texas Children's Fetal Center; ${ }^{3}$ Michael E. DeBakey Department of Surgery and ${ }^{4}$ Department of Obstetrics and Gynecology, Baylor College of Medicine, Houston, TX, USA

Introduction: The MOMS trial demonstrated benefit to open fetal myelomeningocele repair, but wide variation in surgical technique persists between centers. We reviewed our initial experience at Texas Children's Hospital and describe our surgical algorithm for fetal repair. Methods: Between February 2011 and March 2013, 51 women completed presurgical evaluation. Nineteen patients met published criteria; 10 
proceeded to fetal repair. The mean maternal age was 26.5 years (range 19-40); mean gestational age was 24.1 weeks (range 21.3-25); the vertebral defect was L3 or lower in $70 \%$. Fetal positioning was confirmed during a time-out immediately before hysterotomy. After cutting the arachnoid to release the placode, on-lay dural graft was placed and reinforced with epidural sealant. If tension-free primary skin closure could not be achieved, lateral relaxing incisions and/or dermal patch graft was employed.

Results: Primary closure was achieved in seven cases; lateral relaxing incisions were required in one. Two cases required a dermal patch; lateral relaxing incisions were made in one. One fetus suffered severe bradycardia requiring delivery before fetal repair. The mean gestational age at delivery was 33.5 weeks (range 24.337). Among fetuses with completed repair, postoperative MRI demonstrated complete reversal of the Chiari 2 malformation in 7/9 patients $(78 \%)$. Mean post-natal follow-up was 5.4 months. Three patients had CSF leak and two required post-natal intervention for hydrocephalus; both had persistent Chiari malformation on postoperative MRI.

Conclusions: Postoperative MRI demonstrating reversal of the Chiari 2 malformation corresponded with healed fetal repair at birth and no signs of post-natal hydrocephalus during follow-up.

\section{1}

\section{Prenatal in-utero repair of myelomeningocele: Saint Louis} experience

Samer K. Elbabaa ${ }^{1}$, Rachel Elledge ${ }^{2}$, Kurt Eichholz ${ }^{1}$, Edmund Yang ${ }^{3}$, Emanuel M. Vlastos ${ }^{4}$

${ }^{1}$ Division of Pediatric Neurosurgery, Department of Neurosurgery, Saint Louis University School of Medicine, $;{ }^{2}$ Division of Pediatric Neurosurgery, Cardinal Glennon Children's Medical Center,; ${ }^{3}$ Department of Surgery, Saint Louis University School of Medicine,; ${ }^{4}$ Department of Obstetrics, Gynecology and Women's Health, Saint Louis University School of Medicine, Saint Louis, Missouri, USA

Introduction: The Management of Myelomeningocele Study (MOMS) trial results showed improved outcomes in prenatal repair group compared to the postnatal group. We report our initial experience with 21 case series of in-utero myelomeningocele (MMC) repair at the Saint Louis Fetal Care Institute.

Methods: MOMS trial's maternal/fetal inclusion and exclusion criteria were used between May 2011 and March 2013 to screen 37 pregnant female patients, 21 patients underwent the prenatal repair between 20 and 25 weeks' gestational age (GA). We prospectively followed maternal, fetal and neonatal data for our first 21 cases.

Results: All MMC defects underwent successful in-utero repair, with subsequent progression of the pregnancy. Average GA at time of MMC repair was $24+2 / 7$ weeks. Average GA at time of delivery was $34+1 / 7$ weeks. Operative time of microsurgical repair after hysterectomy averaged 34 minutes. Fetal post-repair MRIs were obtained in 12 patients at 30 weeks GA, all showed improvement of hindbrain herniation and ventricular size. One baby died secondary to complications of prematurity. 5 of surviving 20 babies met standard criteria for treatment of hydrocephalus including 2 babies underwent VP shunt placements (10\%) and 3 babies (15\%) underwent successful Endoscopic third ventriculostomy (ETV).

Conclusion: In the post-MOMS trial era, prenatal in-utero MMC repair is becoming a more common procedure with a growing evidence that prenatal vs. postnatal repair may result in improved neurologic outcomes with acceptable morbidity/mortality risks. Our preliminary results support these initial findings, especially with respect to the reduction in the incidence shunt-dependent hydrocephalus. ETV as an alternative to shunting deserves a closer look and may show promising results in the setting of improved hindbrain herniation.

\section{2}

Histological structure of filum terminale in normal human fetuses Yusuf $\mathrm{Izci}^{1}$, Servet Guresci ${ }^{2}$, Cahit Kural ${ }^{3}$, Erhan Arslan ${ }^{4}$

${ }^{1}$ Department of Neurosurgery, Gulhane Military Medical Academy, Ankara, Turkey; ${ }^{2}$ Department of Pathology, Kecioren Research and Training Hospital, Ankara, Turkey; ${ }^{3}$ Department of Neurosurgery, Hakkari Military Hospital, Hakkari, Turkey; ${ }^{4}$ Department of Neurosurgery, Kecioren Research and Training Hospital, Ankara, Turkey

The structure of filum terminale (FT) is important on the development of tethered cord syndrome (TCS) in children. Although many studies were performed on the histological structure of FT in adults, there is no detailed histological investigation in normal human fetuses. The histological examination of FT was performed in 15 normal human fetuses. 11 of them were female and 4 were male. The gestational age of the fetuses was ranged between 14 weeks and 35 weeks. The weight was ranged between $180 \mathrm{~g}$ and $1750 \mathrm{~g}$. The FT of each fetus was cut and examined histologically for fat tissue, fibrous tissue, peripheral nerve, ganglion, ependymal cells, gliosis and collagen types (Type I and III). Fat tissue was observed in one specimen while fibrous tissue was found in 5 specimens. Peripheral nerve was detected in $11 \mathrm{FT}$, ganglion in 6, ependymal cells in 5, gliosis in 7 FT samples. Type III collagen was present in 10 specimens with different concentrations while type I was not detected. Type III collagen was mostly around the vessels and in the perineurium in 3 FT samples. The structure of FT in fetuses is similar with those of adult FT. TCS may probably develop after the birth, but detailed studies are needed to understand the histological basis of TCS in children.

\section{3}

The pathology of lumbosacral lipomas, are there implications for the pathogenesis of tethered cord?

Victoria Wykes ${ }^{1}$, Nicki Cohen ${ }^{2}$, Thomas S. Jaques ${ }^{2}$, Dominic N. Thompson ${ }^{1}$ ${ }^{1}$ Department of Paediatric Neurosurgery, ${ }^{2}$ Department of Neuropathology Great Ormond Street Hospital for Children NHS Foundation Trust, London, UK,

Background: Up to $70 \%$ of children with lumbosacral lipoma have neurological or urological symptoms at presentation or during followup. Mechanical distortion of the terminal spinal cord and nerve roots is the supposed mechanism of deterioration and is the premise underpinning neurosurgical intervention. Scant attention has been given to the malformative aspects of this condition and the role that primary dysgenesis may play in the aetiology of "tethered cord syndrome" is unclear.

Methodology: A histopathological analysis of resected lumbosacral lipomas was undertaken. The lipoma architecture and the presence of additional tissues and cell types were recorded. Lipomas were classified from pre-operative MRI scans.

Results: 55 surgical specimens were reviewed. $25 \%$ contained overlying epidermis and dermis and in approximately half of these an epidermal pit was present. In almost $75 \%$ of cases adipose tissue was not just subdermal but impinged on epidermal adnexal structures. 95\% of cases showed thickened bands of connective tissue associated with peripheral nerve fascicles. 91\% contained blood vessels with enlarged lumina and thickened walls. Additional neuroectodermal derivatives (glial cells, ganglion cells, leptomeninges, ependymal structures and Paccinian-like structures) were encountered in $28 \%$ of cases. Haemangioma, bone and cartilage were also observed. The diversity of tissues and cell types appeared greater amongst complex lipomas (transitional and chaotic) than simpler forms (dorsal and caudal).

Conclusions: The term lumbosacral lipoma underrates the heterogeneity of this congenital anomaly. These findings challenge currently held notions regarding the embryogenesis of spinal lipomas and may have implications for the mechanisms of clinical deterioration. 
14

Functional neuro-urological tests and their relevance in pediatric patients with spinal pathologies related to tethered cord and syringomyelia

Martina Messing-Jünger ${ }^{1}$, Thomas Riedel ${ }^{2}$, Marcus Pauly ${ }^{2}$, Claudia Schmidt $^{1}$, Michael Szallies ${ }^{3}$, Maike Martin ${ }^{1}$

${ }^{1}$ Asklepios Children's Hospital, Department of Pediatric Neurosurgery, ${ }^{2}$ Asklepios Children's Hospital, Department of Pediatric Surgery and Urology, ${ }^{3}$ Asklepios Children's Hospital, Department of Pediatric Radiology, St. Augustin / Bonn, Germany

Objective: Often neuro-urological problems are the only symptoms related to spinal malformations in children. Clinical variations of these disorders are typical. The interpretation of pre- and postoperative results is sometimes difficult, e.g. regarding therapeutical recommendations. In this study we intended to define typical neuro-urological findings and to evaluate our surgical results.

Method: A prospective observational study was performed in order to investigate pre and postoperative follow-up investigations of urinary functions in children with spinal malformations related to tethered cord and syringomyelia. Cystomanometric and uroflow data as well as sonographic pre and postvoiding sonography were screened and correlated with course and surgery data. Additionally patient informations (age, gender, diagnoses, findings/symptoms, therapy) were recorded.

Results: Out of a total of 128 eligible patients we evaluated so far 48 . Major diagnoses were lipomeningocele, myelomeningocele and other tethered cord pathologies. 2 patients were $>18$ yrs during all functional tests. In two thirds of the patients postoperative urinary bladder pressure was reduced after surgery resulting in a higher incidence or frequency of intermittent catheterism in preexisting sphincter-detrusor-dysfunctions. In most of these cases this was correlated with decreased danger of reflux and other complications of a small capacity high pressure bladder. Bladder capacity became smaller again during long-term follow-up.

\section{5}

Midline neuroectodermal appendages or the so-called human tail. Report of a series of 13 patients

J. Francisco Salomão ${ }^{1}$, Heloisa Novaes ${ }^{2}$, Kalil Madi ${ }^{3}$, Antonio R. Bellas ${ }^{1}$ ${ }^{1}$ Division of Pediatric Neurosurgery, ${ }^{2}$ Department of Pathology, Fernandes Figueira National Institute of Health for Children, Woman and Adolescent, Rio de Janeiro, Brazil

Midline neuroectodermal appendages (MNA) are skin protrusions of variable size and form that often resemble tails and are considered rare markers of occult spinal dysraphism. The authors reviewed retrospective the surgical and pathological records of 13 children with 16 MNA. The median age at surgery was six months. Fourteen appendages were found at the lumbo-sacral-coccygeal region and another two at the thoracic and cervical region respectively. Three were multiple. An underlying subcutaneous lipoma was identified in all cases. Images obtained in all patients showed a tethered cord in 12 . Three patients were symptomatic. All appendages were composed of mature fat covered with full thickness skin. According to the surgical and pathological findings, 14 appendages were categorized as follow: 1 . MNA in which subcutaneous fat was continuous to a conus lipoma $(\mathrm{n}=8) ; 2$. MNA in which a lipofibroneural stalk connected the subcutaneous fat to the spinal cord $(n=5) ; 3$. MNA with no connection to the cord $(n=1)$. Six patients had associated intraspinal lesions, most filum fibrolipomas $(n=4)$. The etiology of MNA is debatable and has been ascribed to remnants of the embryionic tail, to superficial extension of dermal sinus tract or to amniotic bands. We show that MNA are invariable continuous with subcutaneous lipomas and that, with one single exception, those were connected to neural structures either through a bulk of mature fat or through a lipofibroneural stalk. Thus, we believe that MNA relates to premature disjunction of the neural tube in the same way as conus lipomas.
16

Tethered cord and spinal cord pulsation in the development of syringomyelia

Uwe Max Mauer ${ }^{1}$, Klaus Efinger ${ }^{2}$, Ulrich Kunz ${ }^{1}$, Chris Schulz ${ }^{1}$

${ }^{1}$ Department of Neurosurgery, German Armed Forces Hospital,;

${ }^{2}$ Department of Radiology, German Armed Forces Hospital, Ulm, Germany

Objective: Tethered cord syndrome is known to cause syringomyelia also in the absence of an obstruction to cranial-to-spinal CSF flow. We conducted this study to investigate this phenomenon in our patient population.

Methods: A total of 877 patients with syringomyelia underwent cardiacgated phase-contrast magnetic resonance imaging (MRI) of CSF flow pulsation at our institution from 2003 to mid-2012. Of these, 35 patients presented with primary tethered cord syndrome, 58 with posttraumatic syringomyelia, and 21 with postoperative syringomyelia (50 women, 64 men; mean age: 39 years, range: $1-76$ years). All together we investigated 114 Patients (17 under the age of 18, 9 under the age of 10).

Results: In patients with primary tethered cord syndrome, CSF flow obstruction similar to that seen in association with intradural adhesions was detected only in the presence of diastematomyelia (12 patients). In patients without tethered cord syndrome, no relevant CSF pulsation was noted below lumbar vertebra 1 (L1). In patients with tethered cord syndrome and a low-lying conus, however, relevant pulsations were also detected in the lumbar subarachnoid space similar to the pulsations seen in the region of the lower thoracic spine in patients without a low-lying conus. For example, in one young man with holocord-syringomyelia and tethered cord syndrome the cavity decreased dramatically after untethering.

Conclusions: Apart from brain pulsation, spinal cord pulsation decisively contributes to the development of syringomyelia in some groups of patients. Cranial pulsation appears to play a less important role in these cases.

\section{7}

Treatment of diastematomyelia in children. We report our surgical experience in the treatment of 30 children with diastematomyelia

Pierre-Aurélien Beuriat, Alexandru Szathmari, Anne-Claire RicciFranchi, T. Jacquesson, C. Rousselle, Carmine Mottolese

Department of Pediatric Neurosurgery, Neurologic and Neurosurgical Hospital Pierre Wertheimer, Lyon, France

Material and Methods: Between 1990 and 2010 we operated 30 patients with diastematomyelia: 11 boys and 19 girls with the age range from 1 month to 16 year-old. The clinical picture has been characterized by an orthopaedic syndrome in 8 cases, by neurological and orthopaedic symptoms in 17 patients and only neurological syndrome in 5 cases. Twelve patients presented cutaneous signs. The radiological investigations consisted in a spinal MRI, a CT-scan focused at the level of the lesion and x-rays of the spinal column. The bone spur was located in the dorso-lumbar region. Six patients were treated for an associated myelomeningocele at birth. All patients benefited of the bonny spur excision.

Results: 9 patients had no neurological deficit and 15 patients improved. 5 others patients were stabilized and 1 patient presented a motor function worsening. Surgery for scoliosis was necessary for only 9 patients. Postoperative complications were represented by a wound infection in four patients, pseudomeningocele in 3 cases and transitory motor deficits in 4 patients. From urological point of view the patients with myelomeningocele presented urinary incontinence; 4 patients presented a post-operative incontinence, 2 patients an urinary retention, 5 patients minor transitory disturbances and 13 patients no urinary troubles. Conclusion: The diastematomyelia represents a particular form of tethered cord and, in our opinion, its surgical treatment is imperative. The early surgical treatment allows scoliosis improvement avoiding surgical correction. The associated myelomeningocele represents an aggravating factor for clinical, orthopaedic, urological and surgical results. 
18

Adult spinal dysraphism: spectrum and outcome

of the disease - analysis of 73 patients

Rakesh Ranjan, Ashis Pathak, Sunil K Gupta, V. K. Khosla

Deaptment of Neurosurgery, Post Graduate Institute of Medical Education

and Research, Chandigarh, India

Introduction: Spinal dysraphism is one of the common congenital nervous system anomaly seen in pediatric neurosurgery. A small percentage of this population may not present during childhood due to multiple reasons. Reports of such patients are scanty in medical literature.

Material and methods: A review of patients presenting with spinal dysraphism between 1964 to 2009 was done and clinical spectrum of patients over 18 years were recorded for their clinical and radiological findings. Various treatments and outcome were analysed.

Results: There were 73 patients who presented after 18 years to the department with complaints related to spinal dysraphism. 24 presented before 21 years of age while 49 were above. There were 41 males and 32 females. 22 patients had symptoms present at birth but did not seek treatment. Swelling in the back was noted in 19, trophic ulcers in 10 and muscle wasting in 5 . The most common presenting features were motor deficits (38/73); sensory deficits (35/73); backache (20/73) and bladder or bowel dysfunction (20). MRI of lumbosacral spine was available in 33 patients while CT scan in 7. Low lying tethered cord was seen in 40 patients, myelomeningocele in 26 and SCM in 12. Epidermoid was seen in 11.36 patients underwent surgery and follow-up was available in 26 patients.

Conclusion: The majority of patients with adult spinal dysraphism suffer from occult variety and tethered cord syndrome. The clinically asymptomatic ones may not still need intervention and neurological improvement is seen in over $50 \%$ patients operated after onset of deficits.

\section{9}

Adult tethered cord: A retrospective study of clinical presentation and indication for surgery

Eveleen Buelens ${ }^{1,3}$, Carlotte Kiekens ${ }^{2}$, Tom Meurrens ${ }^{2}$, Frank van Calenbergh ${ }^{1,3}$

${ }^{1}$ Department of Neurosurgery and ${ }^{2}$ Rehabilitation Science and Physiotherapy, University Hospital Gasthuisberg, Catholic University Leuven, Leuven, Belgium; ${ }^{3}$ Laboratory of Experimental Neurosurgery and Neuroanatomy, Catholic University, Leuven, Belgium

Introduction: Surgery for tethered cord due to lumbosacral lipoma, tight filum and secondary tethering after myelomeningocele repair is usually performed in children. In recent years, tethered cord is recognized more frequently in adults. However surgical indications are unclear and because many patients have long-standing deficits, advantages of surgery may be lower than in children.

Methods: We retrospectively studied medical records of 61 adult patients with tethered cord who consulted the department of neurosurgery between 1996 and 2012. 42 patients were operated, in 19 a conservative management was proposed. The anatomy of the lesion causing tethering was determined. Indications for surgery and reasons for not operating were studied. The data were compared to a similar earlier study in children.

Results: Tethered cord was caused by lipoma in 28 patients, tight filum in 5 patients and was secondary to meningomyelocele repair in 19. Two patients had diastematomyelia, 3 had a dermal sinus, 3 had an epidermoid cyst and 1 patient with the currarino syndrome. Many patients had already long standing deficits: $68 \%$ had sphincter disturbances and $60 \%$ had lower limb motor deficits several years before the diagnosis. Orthopaedic consequences were also frequent: Foot deformities in $41 \%$ and scoliosis in $34 \%$. In $42 \%$ the surgical indication was lumbar and/or sciatic pain, in $30 \%$ deterioration or occurrence of sphincter disturbances.
Conclusions: Most adult patients operated for tethered cord syndrome have long-standing and multiple deficits. The most common indication for surgery is pain. Compared to children, surgery for secondary tethering seems less frequently performed.

\section{Brain Malformation}

\section{0}

Cardiac-gated phase-contrast magnetic resonance imaging and cardiac-gated cine balanced fast-field echo sequences in postoperative patients with Chiari I malformation Uwe Max Mauer ${ }^{1}$, Silke Steinhoff ${ }^{2}$, Ulrich Kunz ${ }^{1}$, Chris Schulz ${ }^{1}$

${ }^{1}$ Department of Neurosurgery, German Armed Forces Hospital of Ulm, ${ }^{2}$ Department of Radiology, German Armed Forces Hospital of Ulm, Ulm, Germany

Object: The causal treatment of Chiari I malformation consists of removing the obstruction of cerebrospinal fluid (CSF) flow at the level of the foramen magnum. CSF flow can be visualized using dynamic magnetic resonance sequences. We investigated the clinical usefulness of two different sequences in patients with Chiari I malformation.

Methods: Ninety patients with Chiari I malformation underwent preoperative MRI of CSF pulsation. Syringomyelia was present in 59 patients and absent in 31 patients. We used cardiac-gated phase-contrast magnetic resonance imaging studies and cardiac-gated cine balanced fast-field echo sequences studies.

Results: The changes in pulsation were highly significant in the region of the (enlarged) cistern $(\mathrm{p}=0.0005)$. Maximum and minimum velocities, i.e. the pulsation amplitude, considerably increased in the region where the syrinx was largest in diameter. It was interesting to note that the changes of pulsation in these patients were significant in the subarachnoid space in all spinal segments but not in the syrinx itself and in the central canal.

Conclusions: The demonstration of CSF flow pulsation is a useful tool for assessing surgical outcome. The results presented here, however, question current theories on the pathogenesis of syringomyelia.

\section{1}

Can children with asymptomatic sylvian arachnoid cysts play ball? Matthieu Vinchon, Marc Baroncini

Department of Pediatric Neurosurgery, University Hospital, Lille, France

Background and purpose: Asymptomatic sylvian arachnoid cysts (ASAC) are a common finding in children and the risks of clinical progression and hemorrhagic complication is not established; in consequence, many children are denied access to contact sports, although the scientific basis of these precautionary measures is lacking.

Material and methods: It is our policy to allow all sport activities in children diagnosed with ASAC and not to prescribe systematic control imaging. We retrospectively reviewed cases of ASAC seen in pediatric neurosurgical clinic since 2002, and reviewed all available imaging. In addition, we contacted their families by phone call in order to check their health status, and whether complications occurred.

Results: We reviewed 42 cases of ASAC, with a cumulated follow-up of 124 years. During that period, none of the patients had any cyst-related clinical worsening. Twenty-three had control imaging, either systematically prescribed by their family doctor, or because of symptoms, which were finally considered cyst-unrelated. The cumulated duration of radiological follow-up was 76 years; in $21 / 23$, the size of the cyst was unchanged; in 2, it increased slightly, but did not require treatment. None of our cases of ASAC had hemorrhagic complications.

Conclusion: Our study shows that the risk of complication of ASAC is certainly very small. In consequence, these lesions should be held as 
incidental findings not requiring further medical attention. Considering the long-term health risks associated with idleness, we think that children with ASAC should be encouraged to practice all sport activities.

\section{2}

\section{Subdural hematomas caused by arachnoid cysts}

Matthieu Vinchon, Marc Baroncini

Department of Pediatric Neurosurgery, University Hospital, Lille, France

Background and purpose: Although subdural hematoma (SDH) is a common presentation of arachnoid cysts (AC), few series document their natural history and surgical outcome in children.

Material and methods: We reviewed retrospectively children treated for intracranial AC since 1980, and selected cases revealed by a SDH. The treatment, drainage of either the cyst or the $\mathrm{SDH}$, varied according to the period and the surgeon.

Results: Among 247 cases of AC, SDH was the presenting feature in 45 cases, associated with significantly older age (mean 109.6 VS 70.2 months) and higher M/F ratio (4.0 VS 2.05)). The cyst was sylvian in 41 cases, at the convexity in 3 , and interhemispheric in one. In the majority of cases, no or only mild trauma was identified, and only 4 were sports-related. Treatment was associated with a high rate of failure, significantly more with drainage of the cyst $(9 / 11)$ compared with drainage of the SDH (14/32),

Conclusion: AC are an important cause of SDH in children. Our data suggest the presence of a SDH at presentation attests of impaired CSF absorption. Drainage of the cyst itself is thus doomed to failure, and drainage of the SDH should be preferred. Since trauma was generally mild or absent in cases of $\mathrm{CA}$ with $\mathrm{SDH}$, we consider that children with incidental AC should practice sports. Understanding why many individuals with $\mathrm{AC}$ in the population remain asymptomatic while some develop brain compression, and others a $\mathrm{SDH}$, may hold the key to a better understanding of CSF circulation.

\section{3}

Communicating hydrocephalus after primary microsurgical fenestration of symptomatic middle fossa arachnoid cysts in children Anton E. Korshunov, Yury V. Kushel

Pediatric Neurosurgery department \#2, N.N. Burdenko Nerosurgery Institute, Moscow, Russia

Introduction: We describe intracranial hypertension (ICH) arising within weeks after microsurgical fenestration (MSF) of symptomatic middle fossa arachnoid cysts (MFAC) in children.

Methods: Records of 40 children who had MSF of their symptomatic MFAC as a primary treatment done at our department from 2002 to 2012 were reviewed. The most common presentations were progressively large head (21) and recent onset headache (15). M/F ratio was 29/11, age ranged 3-192 months (median 34). There were 37 Galassi-3 and only 3 Galassi-2 cysts. Follow-up ranged 2-120 months (median 14; available for 37 patients).

Results: 7 of 16 patients under 2 years of age (43\%) and 1 of 24 older children (4\%) acutely developed symptoms of ICH 7-30 days (median 20) after surgery. At deterioration cysts disappeared on CT in 2 and reduced in 6 patients. Ventricles remained narrow in 2 and dilated compared to preoperative studies in 6 patients (with periventricular edema (PVE) in 3). Lumbar puncture done in 6 patients discovered hypertension, normal cell count and negative cultures. 4 patients improved after VP-shunts, 1 patient improved after LP-shunt. Two patients with narrow ventricles received CP-shunts, both later developed ventricular enlargement with PVE which necessitated conversion to VP-shunt. 1 patient was managed on dexamethasone and avoided a shunt. Apart from these only one patient received a shunt in this series which occurred 40 months after initial surgery.
Conclusion: ICH arising within weeks of MSF of MFAC is due to communicating hydrocephalus and is common in children under 2 years.

\section{4}

Endoscopic treatment of suprasellar arachnoid cysts

Jürgen Krauss, Niels Sörensen, Tilmann Schweitzer

Section for Pediatric Neurosurgery, Neurosurgical Department, University of Würzburg, Germany

Introduction: Endoscopic fenestration has become the treatment of choice for suprasellar arachnoid cysts (SSAC).Discussion remains open about the necessary extent of the procedure and outcome analysis could still use a broader data base.

Method: Analysis of a single institution series and review of series published with more than one hundred patients combined.

Results: Between 1996 and 201230 consecutive patients with SSAC were endoscopically treated, 26 children with an average age of 3 years 11 months and 4 adults. 29 patients had ventriculomegaly, 14 of them presenting with symptoms of raised ICP. 11 patients had endocrine symptoms. To prevent stoma occlusion by redundant cyst wall, we resected the dome subtotally in 20 patients and partially in 9 . Basal cyst wall fenestration was performed in 29 cases, a slit valve was observed in 16 patients. Complications included one ventriculitis and a transient oculomotor palsy. No permanent morbidity or mortality occurred. Three patients required shunting despite adequate CSF communication; all were infants with highly active hydrocephalus. Endocrine symptoms were unaltered by surgery, long-standing mental or memory deficits resolved less well than symptoms of shorter duration.

Conclusion: Endoscopic fenestration of SSAC is highly efficient in all surgical variants; basal fenestration appears to add to the reliability of the success. The benign course of asymptomatic cysts has led to recommendations of observation only. Our experience with irreversible deficits in very large cysts in older children and adults suggest that such large cysts be treated early even when asymptomatic at that stage.

\section{5}

Intracranial pressure parametres in children and adolescents with Chiari malformation type 1

Radek Fric, Bernt J. Due-Tønnessen, Per Kristian Eide

Department of Neurosurgery, Rikshospitalet, Oslo University Hospital, Oslo, Norway

Objective: to evaluate the value of preoperative assessment of intracranial pressure in young patients with Chiari malformation type 1 (CMI).

Material and Methods: 38 children and adolescents (range 1 months -18 years) were treated for Chiari malformation type 1 by foramen magnum decompression and, in some cases, a CSF derivational procedure, during period from 2008 - 2012. In 19 of them, the continuous intracranial pressure (ICP) monitoring was undertaken as a part of preoperative work-up or in case of clinical or radiological progression despite surgical treatment. The static ICP was characterized by mean ICP while the pulsatile ICP was determined from cardiac beat-induced single ICP waves and expressed by the ICP puls pressure amplitude and latency.

Results: Generally, there was found no correlation between pulsatile ICP parameters and the degree of tonsillar ectopy into foramen magnum, presence of syringomyelia or radiological evidence of hydrocephalus. Furthermore, intracranial pressure parameters do not always normalize simply after restoration of cerebrospinal fluid (CSF) pathways by surgical decompression of foramen magnum.

Conclusion: Pulsatile ICP parameters reflect impaired intracranial compliance in case of Chiari malformation better then static ICP. Patients with significant disturbance of CSF circulation should be treated by CSF derivational procedures prior to decompression of foramen magnum. Thus, we find continuous ICP monitoring with evaluation of pulsatile 
pressure to be an important step in preoperative work-up of patients with CMI and suspected impaired CSF dynamics.

\section{6}

The risk factors for and the influence of hydrocephalus on neurological outcome in children born with an encephalocele Stephanie Da Silva, Yasser Jeelani, Andrew Yousef, Mark D. Krieger, J. Gordon McComb

Division of Neurosurgery, Children's Hospital of Los Angeles, Los Angeles, CA Department of Neurological Surgery, Keck School of Medicine, University of Southern California, Los Angeles, CA, USA

Introduction: There is a known association of hydrocephalus with encephaloceles. Risk factors for hydrocephalus and the neurological outcome were reviewed in our series of patients born with an encephalocele. Methods: Under IRB approval, a retrospective analysis was undertaken of patients treated for encephaloceles at a single institution between 1994 and 2012.

Results: 70 children ( 38 female) were identified. The median age at presentation was 2 months (range $0-71 \mathrm{~m}$ ). Mean follow-up was $2.6 \mathrm{y}$ (range $0-18 y$ ). Location was anterior (14 children) or posterior (56) to the coronal suture. The average size was $4 \mathrm{~cm}$ (range $0.5-23 \mathrm{~cm}$ ). Fortyseven contained neural tissue. Eight infants presented at birth with CSF leaking from the encephalocele, with one being infected. Six patients presented with hydrocephalus while 11 developed hydrocephalus postoperatively. The following factors had a statistically significant association with hydrocephalus: contents $(\mathrm{p}=0.04)$, size $(\mathrm{p}=0.01)$, and associated anomalies $(\mathrm{p}=0.01)$. Fourteen had severe developmental delay, 9 moderate, and 19 mild; 28 patients were neurologically normal. Encephaloceles of $\geq 2 \mathrm{~cm}$ diameter $(\mathrm{n}=38 / 56, \mathrm{p}=0.01)$, presence of hydrocephalus $(\mathrm{n}=17$ / $17, \mathrm{p}<0.01)$ or associated CNS anomalies $(\mathrm{n}=32 / 43, \mathrm{p}=0.03)$ had a high incidence of developmental delay.

Conclusion: The incidence of hydrocephalus in children born with encephaloceles is associated with contents of the encephalocele, size, and associated anomalies. Neurological outcome in children born with encephaloceles is associated with size, associated anomalies, and incidence of hydrocephalus. In contrast to modestly good neurological outcome in children with an encephalocele without hydrocephalus, the presence of hydrocephalus resulted in a far worse neurological outcome.

\section{7}

Surgical treatment of transsphenoidal encephaloceles: transpalatal versus combined transpalatal and transcranial approach Hideki Ogiwara, Nobuhito Morota

Department of Neurosurgery National Center for Child Health and Development, Tokyo, Japan

Background: The optimal surgical treatment of transsphenoidal encephaloceles (TSECs) is controvertial.

Objective: TSEC is a rare developmental anomaly with herniation of neural elements including pituitary gland or optic apparatus, through a defect of sphenoid bone. In order to prevent obstructive dyspnea, endocrine dysfunction, or visual disturbance, surgical correction was usually performed. However, the optimal operative approach has not been determined yet. The authors present their surgical experiences with transpalatal, transcranial, or combined approach.

Methods: We retrospectively analyzed surgical results of patients with TSEC. The charts were reviewed for demographic data, clinical presentation, surgical therapy, and clinical outcomes.

Results: Seven consecutive patients with TSEC were surgically treated from March 2004 to February 2012. The mean age was 23.9 months (ranged from 2 to 68 months). Four patients with TE with a bone defect within the sphenoid bone were successfully treated by transpalatal approach. Two patients with an extensive sphenoethmoidal bone defect treated either by transpalatal or transcranial approach experienced several relapses with a prolapse of the encephalocele. One patient with an extensive sphenoethmoidal bone defect was successfully treated by combined transpalatal and transcranial approach without relapse for 3.6 years. Conclusion: Combined transpalatal and transcranial approach is useful for TSEC with an extensive sphenoethmoidal bone defect to dissect the sac and expose the bone defect circumferentially. If dissection is not sufficient, relapse with a prolapse of the encephalocele can ensue. Transpalatal approach is sufficient for TSEC with a bone defect limited within the sphenoid bone.

\section{8}

Imaging diagnosis and prognosis of communicating interhemispheric cysts

Atsuko Harada ${ }^{1}$, Hidetsuna Utsunomiya ${ }^{2}$, Takumi Yamanaka ${ }^{1}$, Naoki Kagawa $^{3}$, Masahiro Nonaka ${ }^{4}$, Kenichi Nishiyama ${ }^{5}$, Reizo Shirane ${ }^{6}$, Mami Yamasaki ${ }^{1}$

${ }^{1}$ Department of Pediatric Neurosurgery, Takatsuki General Hospital, Takatsuki, Japan; ${ }^{2}$ International University of Health and Welfare; ${ }^{3}$ Department of Neurosurgery, Osaka University Graduate School of Medicine, Osaka, Japan; ${ }^{4}$ Department of Neurosurgery, Osaka National Hospital, Osaka, Japan; ${ }^{5}$ Department of Neurosurgery, Brain Research Institute, University of Niigata, Niigata, Japan; ${ }^{6}$ Department of Neurosurgery, Miyagi Children's Hospital, Sendai, Japan

Objective: Interhemispheric cyst (IHC) with callosal dysgenesis and dorsal cyst of holoprosencephaly are two major anomalies with dorsal midline cystic lesions. Due to a significant difference in their prognosis and treatment, the precise diagnosis is of vital importance. IHC has been classified into two types on the basis of whether cystic lesions communicate with the ventricle (communicating IHC; C-IHC) or not (non-communicating IHC; NC-IHC). Although there are a number of reports on the treatment and prognosis of $\mathrm{NC}-\mathrm{IHC}$, such information is limited for $\mathrm{C}$ IHC. The purpose of this study was to investigate the prognosis of C-IHC. Materials and methods: Subjects were 10 cases of C-IHC that was diagnosed using prenatal $(n=5)$ or postnatal $(n=5)$ MRI. We retrospectively reviewed their MRI findings and prognosis.

Results: In 1973, Probst classified C-IHC into two types such as primary CIHC with hypoplastic falx cerebri and secondary C-IHC with intact falx. According to this classification, seven cases were diagnosed as primary $\mathrm{C}$ $\mathrm{IHC}$ and the remaining three cases were as secondary C-IHC. In total, 8 cases underwent ventriculo-peritoneal shunt. For primary C-IHC, the prognosis was fair in 4 cases, but was poor in 2 cases with cortical dysplasia. Pregnancy was terminated in 1 case. Secondary C-IHC was associated with myelomeningocele $(\mathrm{MMC}, \mathrm{n}=2)$ and microcephaly $(\mathrm{n}=1)$. The prognosis was fair in one MMC case, but was poor in the other 2 cases.

Conclusions: There was no clear difference in prognosis between primary $\mathrm{C}$-IHC and secondary C-IHC. Overall, their prognosis was fair in half of the cases. A critical factor determining prognosis was anomalies associated with C-IHC. Further studies are necessary to establish a prognosis-oriented classification.

\section{9}

Neuro-endoscopic treatment of sylvian arachnoid cysts in children Marina Brigui, Federico Di Rocco, Thomas Blauwblomme, Stephanie Puget, Christian Sainte-Rose, Michel Zerah

Department of Paediatric Neurosurgery, Hôpital Necker-Enfants Malades, Paris, France

Surgical management of sylvian arachnoid cysts in the paediatric population is highly controversial. The aim of our study is to assess the effectiveness and safety of the neuro-endoscopic treatment of sylvian cysts. Between 2005 and 2012, we have treated 32 symptomatic children with a mean age of 4.55 years (ranging from 4 months to 18 years). Two to 3 stomia were performed through a temporal burr hole. Surgery was uneventful in all but 
one child in which an intracystic hemorrahge occurred that prevented to perform multiple stomias. Six patients presented with post-operative subdural collections, three of them needing temporary drains. At last follow-up control (mean follow-up period of 4.4 years) 28 patients had significant improvement of their symptoms. However, 2 children experienced a recurrence due to closure of the fenestrations; one child underwent a successful second endoscopy and the other the placement of a cystoperitoneal shunt. We conclude that the endoscopic treatment of arachnoid cysts of the temporal fossa is a safe and durable method. The incidence of recurrences and postoperative subdural collections is not superior to the microsurgical approach, with the benefit of a less invasive procedure.

\section{0}

Aplasia cutis congenita: about 10 cases treated by skin expansion Blandine Grassiot, Alexandru Szathmari, Pierre-Aurélien Beuriat, Anne-Claire Ricci-Franchi, C. Rousselle, Carmine Mottolese Service de Neurochirurgie pédiatrique E, Hôpital Pierre Wertheimer, Groupement hospitalier EST, Hospices civils de Lyon, Bron, France

Introduction: Aplasia cutis congenita (ACC) represents a group of different malformations characterised by a skin defect localised essentially on the scalp. Different stage exists according to the localisation, the number and extension or the associated malformative syndromes. When the defect is small, the treatment is easy but when it is large and associated with a skull defect, rapid closure of the skin in necessary order to avoid complications like infections or haemorrhage.

Methods: In our paediatric neurosurgical service, we treated 10 children with a large ACC and skull associated defect. The treatment consisted in a standardised protocol with systematic sterile draping and moistening of the dura and the use of surgically inserted skin expanders to accelerate the skin growth and close the skin as soon as possible.

Results: This standardised approach permit to fix the skin defect without a skull reconstruction for all the children. None of the children presented complications and the scars had a good evolution. None of the children need a skull reconstruction later.

Conclusion: ACC is a rare and severe affection. An early surgical treatment permits to avoid the main complications as infection and haemorrhage. The skin expansion is the better way to obtain a rapid skin closure.

\section{Craniofacial Surgery I}

\section{1}

Expression of the Bardet-Biedl Syndrome gene 9 (BBS9) in calvarial suture of nonsyndromic craniosynostosis: pathophysiology of primary cilium in suture morphogenesis Wanda Lattanzi ${ }^{1,2}$, Gianpiero Tamburrini ${ }^{3}$, Camilla Bernardini ${ }^{1}$, Luca Massimi $^{3}$, Marta Barba ${ }^{1}$, Fabrizio Pignotti ${ }^{3}$, Maria C. Geloso ${ }^{1}$, Massimo Caldarelli $^{3}$, Fabrizio Michetti ${ }^{1,2}$, Concezio Di Rocco ${ }^{3}$

${ }^{1}$ Institute of Anatomy and Cell Biology, ${ }^{2}$ Latium Musculoskeletal Tissue Bank, ${ }^{3}$ Pediatric Neurosurgery, Università Cattolica del Sacro Cuore, Rome, Italy

Introduction: It is actually more and more clear that non syndromic craniosynostosis (NCS), have a strong genetic background. One of the most recent advances in this context has been the association of sagittal NCS (sNCS) with sequence variants at the BBS9 gene locus, a component of the BBBSome involved in the primary cilium-related signaling. Interestingly, genes causing at least two craniosynostosis syndromes (Carpenter and Sensenbrenner syndrome) are involved in the cilia formation and function, thus suggesting a role for the primary cilium-related signaling in skull morphogenesis.

Methods and results: An in-depth in silico analysis of previous microarray data of 8 patients operated for midline NCS at the Catholic University Medical School, Rome, identified a relevant number of ciliomeassociated genes differentially expressed in fused-versus-patent sutures.
BBS9 was among the most significantly up-regulated genes in the list; this data was confirmed in tissues and cells from an independent set of 20 operated on NCS patients. Confocal microscopy in this context documented that fused suture-derived cells have a decreased tendency to form primary cilia. In addition, the expression of BBS9 during rat suture ossification increased progressively over time.

Conclusions: These data seemed to confirm an active role of BBS9 in osteogenesis and may suggest that BBS9 sequence variants associated to a dysregulation of BBS9 expression could lead to premature suture ossification in NCS.

32

Foramen Magnum morphology contribution to syringomyelia: Evidence for a mechanism akin to "hernia strangulation" in syndromic craniosynostosis Guirish A. Solanki ${ }^{1,2}$, Pasquale Gallo ${ }^{1}$, Magda Garzon ${ }^{1}$, Paul Davies ${ }^{3}$ ${ }^{1}$ Birmingham Children's Hospital, ${ }^{2}$ University of Birmingham and ${ }^{3}$ Institute of Child Health, Birmingham, UK

Introduction: The incidence of Chiari I in syndromic craniosynostosis (SCS) is not well known and the mechanism whereby syringomyelia occurs is poorly understood.

Aims: We describe the frequency of Chiari I Hindbrain Hernia (CMI) and syringomyelia in SCS and evaluate the critical importance of the foramen magnum (FM) morphology in determining the development of syringomyelia in the presence of CM.

Subjects: 106 children with SCS between 2003-2012 were investigated for the presence of CMI \& Syringomyelia. 81 (62\% boys) children, median age 3 ( 5 days-20) years had adequate imaging. 30 age $\&$ sex matched controls were also evaluated.

Methods: MRI measurements included surface area of the FM(SAFM), CMI extent, syringomyelia. Statistical inference obtained.

Results: $39 \%$ had CMI and 10\% Syringomyelia. Crouzon's had the largest incidence of CMI (58\%) \& Syringomyelia (20\%). CMI averaged 9.7 without and $13.8 \mathrm{~mm}$ with syrinx. SCS had greater FM dimensions than controls. A larger SAFM of between 4-to-137\% was noted with CMI vs. controls. Syringomyelia on the other hand was associated with 10$35 \%$ smaller SAFM in all SCS groups. The ratio of SAFM $/ \mathrm{R}+\mathrm{L}$ hernia was below $28 \%$ in $85 \%$ of Syringomyelia cases.

Conclusion: In syringomyelia, hindbrain hernia was greater $(13.8 \mathrm{~mm})$. SAFM is larger in children SCS and CMI alone than with syringomyelia (tight FM). The ratio of SAFM by total CMI descent when $<28 \%$ is predictive of syrinx. This preliminary finding is in keeping with a mechanism of "hernia strangulation" which may apply to syringomyelia.

\section{3}

Is lumbar puncture a reliable measure of intracranial pressure? Usiakimi Igbaseimokumo, Cathy Cartwright, Gregory Hornig, Christian Kaufman

Section of Neurosurgery, Children's Mercy Hospital, Kansas City, Missouri, USA

Introduction: A lumbar puncture as a means of measuring intracranial pressure (ICP) is limited by being a single time point evaluation. A microsensor placed in the brain is considered the gold standard because it is not affected by posture and it allows the child to mimic home activity over a longer period of time. A study was therefore carried out to investigate the reliability of lumbar puncture when compared to intracranial pressure monitor with microsensor device for elective evaluation of suspected intracranial hypertension.

Methods: All patients who had both a lumbar puncture and ICP monitor were reviewed over a three year period. Demographic data, indication for the ICP monitor placement including clinical features, primary diagnosis and the lumbar puncture pressure and the intracranial pressure monitor pressures were collected and analyzed for this study. Statistical analysis was with SPSS v 20 accepting a p-value of less than 0.05 as significant. 
Results: There were 12 children (7females: 5males) aged 2 - 19yrs (mean 11.1 years). The mean ICP measured by lumbar puncture was $22 \mathrm{mmHg}$ (range 18-26) and the mean ICP as measured with the microsensor (Camino bolt) was $7 \mathrm{mmHg}$ (range $3-12, \mathrm{p}<0.0001$ ). The primary diagnosis was craniosynostosis in 7 patients, hydrocephalus in 4 patients and pseudotumor in 1 patient. All patients had headache and there was no papilledema. The anticipated surgical intervention was avoided in all the patients.

Conclusions: This study found a significant difference in the ICP measured by lumbar puncture and ICP monitor. We would recommend the use of ICP monitor in the evaluation of possible chronic raised intracranial pressure rather than a lumbar puncture.

\section{4}

Intracranial pressure increase up to 60 months of age in 217 children with craniosynostosis

Soo Han Yoon ${ }^{1}$, Dong Ha Park ${ }^{2}$

${ }^{1}$ Department of Neurosurgery, ${ }^{2}$ Department of Plastic and Reconstructive Surgery, Ajou University School of Medicine, Suwon, Korea

Introduction: There have been many reports to date that describe the increase in intracranial pressure (ICP) in patients with craniosynostosis, but these have mainly focused on increased ICP in syndromic craniosynostosis conditions, while nonsyndromic craniosynostosis shows an relatively infrequent increase in ICP. Recent observations have suggested hat the increased ICP in nonsyndromic craniosynostosis is more frequent than previously recognized, and thus we report a series of 217 craniosynostosis children with respect to frequency of increased ICP, and age.

Methods: A total of 217 craniosynostosis children underwent preoperative and postoperative ICP measurements with lumbar puncture and classical and trans-sutural distraction osteogenesis over the past 7 years at the Ajou University Hospital, and relevant factors including age, and craniosynostosis type were analyzed.

Results: The mean age of the 217 craniosynostosis study patients was $19.2 \pm$ 20.1 months, the pre-operative mean ICP was $24.7 \pm 9.3 \mathrm{cmH}_{2} \mathrm{O}$, and the post-operative mean ICP was $17.4 \pm 5.2 \mathrm{cmH}_{2} \mathrm{O}$, demonstrating statistically significant difference regardless of craniosynostosis type $(p<0.01)$. The number of patients showing increased pre-operative ICP of greater than 20 $\mathrm{cmH}_{2} \mathrm{O}$ was 148 (68.2\%). The pattern of pre-operative ICP change according to age showed gradual increase with age and reaching a peak at 60 months. Conclusion: Increased ICP is highly prevalent in craniosynostosis children, and since the increase occurs up to 60 months of age, and it is suggested that careful attention should be given to increased ICP in craniosynostosis children with regular testing and examination up to 5 years of age.

\section{5}

Treatment results of fronto-orbital advancement by a distraction osteogenesis for infants with syndromic craniosynostosis Hiroaki Sakamoto ${ }^{1}$, Yasuhiro Matsusaka ${ }^{1}$, Yusuke Watanabe ${ }^{1}$, Ryoko $\mathrm{Umaba}^{1}$, Keisuke Imai ${ }^{2}$, Kazuaki Yamaguchi ${ }^{2}$

${ }^{1}$ Department of Pediatric Neurosurgery, ${ }^{2}$ Department of Plastic and Reconstructive Surgery, Osaka City General Hospital, Osaka, Japan

Purpose: Re-expansion of the cranial vault is often necessary in infants with syndromic craniosynostosis after initial expansion in the standard procedures because of retraction of the expanded bone flaps and of expansion limited by the overlying scalp. Fronto-orbital distraction (FOD) might overcome these disadvantages. We analyzed long follow-up results of FOD applied for infants (age $<1$ year) with syndromic craniosynostosis. Methods: We have treated 51 children with syndromic craniosynostosis from 1998 to 2011. Out of 37 infants who underwent FOD at the age ranged from 3 to 10 months, 20 were Apert, 8 Crouzon, 5 Pfeiffer ( 4 cloverleaf skull) and 4 other types of syndromic craniosynostosis. The frontal bone flap with supraorbital bar was gradually advanced up to the normal depth of the frontal base at the age of 2 years. High intracranial pressure was controlled by shunting for hydrocephalus or by radical strip craniotomy of the fused coronal sutures before 3 months of age. Patients were followed from 1 to 15 years (mean: 7 years) after surgery.

Results: Volumetric study using CT in 10 patients who were followed for more than 3 years after surgery indicated that the intracranial volume was increased to the normal range. Three patients ( 2 Apert and 1 cloverleaf skull) underwent re-expansion in 8, 10 and 23 months after initial FOD. We had re-installation for the dislodged distraction devices in 2 patients and evacuation of epidural abscess in 1 . Two children with severe cloverleaf skull were expired.

Conclusion: These results suggest that FOD applied for infants with syndromic craniosynostosis can provide a near normal development of the cranial volume, resulting in a lower incidence of re-operation for cranial expansion.

\section{6}

Evaluation of the efficacy of decompressive cranioplasty applied to mild metopic suture synostosis with developmental disorders using developmental and psychological examinations

Kazuaki Shimoji ${ }^{1}$, Akihide Kondo ${ }^{1}$, Takaoki Kimura ${ }^{1,2}$, Takeyoshi Shimoji ${ }^{3}$, Masakazu Miyajima ${ }^{1}$, Hajime Arai $^{1}$

${ }^{1}$ Department of Neurosurgery, Juntendo University School of Medicine, Tokyo, Japan; ${ }^{2}$ Department of Pediatric Neurosurgery, Charité Universitätsmedizin, Berlin, Germany; ${ }^{3}$ Department of Neurosurgery, Okinawa prefectural southern medical center / children's medical center, Okinawa, Japan

Objective: Recently, We have applied decompressive cranioplasty for the treatment of mild metopic suture synostosis with developmental disorders. To evaluate the effectiveness of this surgery, we had evaluated these children with several developmental and psychological examinations. Method: 28 children ( 26 boys and two girls) who have developmental disorders with metopic suture synostosis underwent 5 different tests a) Developmental Quotient (DQ) calculated with Kyoto Scale of Psychological Development 2001(KSPD) b) National Rehabilitation Center S-S test(NRC SS test) c) Child Behavior Checklist (CBCL) d) Pervasive Developmental Disorders Autism Society Japan Rating Scale (PARS) a moderate test based on Childhood Autism Rating Scale (CARS) e) Mother's nurturing behavior question sheet in 3 different periods (before surgery, 3 months post surgery and 6 months post surgery). Multiple comparison using Turkey-Kramer method was applied for statistical evaluation.

Results: Improvement was seen statistically significantly in DQ examed with KSPD, expression of words measured with NRC SS test, Behavior factors measured with CBCL, Scores in PARS and increase of positive factors in nurturing scores in behavior questions sheet.

Discussion: Historically, surgical indication of single suture synostosis was considered as cosmetic reasons. However, several reports had shown that the population of developmental disorders with single suture synostosis may have been larger than estimated. The results in this cohort may lead an idea that decompressive cranioplasty may have play an important role to support the improvement of the developmental delay of these patients.

\section{7}

Misinterpretation of signs of bone immaturity in skull x-rays of sagittal craniosynostosis

Tilmann Schweitzer, Hartmut Collmann, Beatrice Jager, Jürgen Krauss Section of Pediatric Neurosurgery, Department of Neurosurgery, University of Würzburg, Germany

Introduction: Beaten copper skull in skull $\mathrm{x}$-rays is regarded as sign for rised intracranial pressure. But it has to be separated from the so called honey comb pattern which is regarded as a sign for immature bone 
formation, often apparent in craniosynostosis cases. Judgement of skull xrays can have several implications such as operative therapy due to assumed rised intracranial pressure.

Methods: We analyzed skull x-rays and the original radiologists' interpretations performed in children with premature sagittal craniosynostosis presenting to our outpatient clinic in the year 2012.

Results: In the year 2012 we saw 140 children with sagittal craniosynostosis at all ages in our outpatient clinic. Among these were 29 first presentations. A skull x-ray was performed in 16 of these children, 7 of these $(44 \%)$ were diagnosed as having raised intracranial pressure because of perceived convolutional markings.

Conclusion: Because of its possible therapeutical implications it is important in skull $\mathrm{x}$-rays to separate true signs of rised intracranial pressure from signs of immature bone. Areas of dysplasia are often misinterpreted as signs of rised intracranial pressure.

Furthermore one has to be aware that over time the signs of dysplasia do resolve while signs of raised ICP may evolve.

\section{8}

\section{Troubleshooting distraction osteogenesis for craniosynostosis}

Takuya Akai ${ }^{1}$, Hideaki Iizuka ${ }^{1}$, Tuyoshi Tsukada ${ }^{1}$, Yasuo Sasagawa ${ }^{1}$, Syunsuke Shiraga ${ }^{1}$, Kazuya Okamoto $^{1}$, Osamu Tachibana ${ }^{1}$, Masahiko Yamashita $^{2}$, Shigehiko Kawakami ${ }^{2}$

${ }^{1}$ Department of Neurosurgery, ${ }^{2}$ Plastic and Reconstructive Surgery, Kanazawa Medical University, Ishikawa, Japan

We reported that distraction osteogenesis is less invasive, and gives greater skull advancement compared to conventional cranioplasty (Pedatr Neurosurg 2006). In this study, we analyzed patients treated by distraction osteogenesis, and identified technical problems and solutions.

Patients: We operated 21 patients, 5 syndromic and 16 non-syndromic. Age at operation was 6 mo. to 13 years in syndromic patients and $5 \mathrm{mo}$. to 4 years in non-syndromic patients. Distraction distances were 18 to $25 \mathrm{~mm}$ and 12 to $25 \mathrm{~mm}$ respectively. During treatment, we encountered several technical problems: i) dural laceration during craniotomy (2 cases): repaired by suturing with fascia; ii) skull fracture at spheono-frontal or coronal sutures ( 2 cases): completed distraction; iii) device dislocation during distraction (1 case): we secured the device to the skull with wires at shaft cutting; iv) wounds around shaft and device ( 2 cases): treated with antibiotics or protective tape.

Discussion: 1) Care is needed to avoid dural damage at frontal bottom burr holes. 2) Completion of craniotomy should be confirmed by checking if bone flap moves in accordance with brain pulsation. Craniotomy line should be placed forward of coronal sutures. 3) For patients younger than 2 years, we recommend a clamp-type device. 4) Shaft should be cut short enough to prevent tip from exerting pressure on scalp from beneath.

Conclusion: Distraction osteogenesis technique has problems that may not occur in a conventional cranioplasty. Problem resolution and advanced procedures development are essential.

\section{9}

Adequate application of distraction osteogenesis to non-syndromic craniosynostosis surgery from analyses of complications in long-term followed-up

Yuichiro Nonaka $^{1}$, Takeshi Miyawaki ${ }^{2}$, Ai Masumoto ${ }^{1}$, Shizuo $\mathrm{Oi}^{3}$, Yuichi Murayama ${ }^{4}$

${ }^{1}$ Division of pediatric neurosurgery, Department of neurosurgery, Jikei University, Tokyo, Japan; ${ }^{2}$ Department of plastic surgery, Jikei University, Tokyo, Japan; ${ }^{3}$ St. Thomas University, Hyogo, Japan; ${ }^{4}$ Department of neurosurgery, Jikei University, Tokyo, Japan

Background: Distraction osteogenesis (D.O.) has changed the way of craniofacial surgery from scanty to significant expansion of craniofacial volume in minimally invasive way. However, D.O. sometimes conduces to unexpected aesthetic results because of preoperative deformities and difficulty in imaging a vector of expansion. We investigated an adequate D.O. application for non-syndromic craniosynostosis surgery from analyses of our series.

Materials and methods: Nine patients ( 5 boys and 4 girls) with nonsyndromic craniosynostosis were enrolled. 4 patients suffered from coronal suture synostosis, and 5 multiple including coronal sutures. D.O was applied as initial surgery for 6 patients and second for 3 , and as frontal advancement (FA) in 6, total calvarial reconstruction (TCR) in 2, hybrid front-orbital advancement (HFOA) in 1. HFOA was composed of reshaped-orbital-bar fixation and FA. All patients underwent neuroimaging included three-dimensional computed tomography.

Results: Mean age at operation was $2 \mathrm{yrs} 8 \mathrm{mos}$, and median followed-up period was $9 \mathrm{yrs} 4 \mathrm{mos}$. Minor skin troubles such as skin score and the exposed device were observed in 3 cases until when all devices were removed. Unfavorable expansion like horns and protruding aside was occurred in 4 patients who underwent TCR and hemi FA.

Discussions and Conclusions: D.O. has contributed to the dramatically change of strategies for craniofacial surgery, however, closely attention to aesthetic morphological appearance should be required when applying this method. From this point of view, brachcephaly with minor distortion could be one of a good candidate for D.O., and HFOA also could be a TCR technique for the severe distorted craniosynostosis.

40

New bioactive glass - fiber-reinforced composite implant for large cranial defects of pediatric cases

Willy Serlo, Jaakko Piitulainen, Ville Vuorinen, Kalle Aitasalo, Pekka Vallittu

Oulu University and Turku University, Oulu and Turku, Finland

Background: Conventional reconstruction of large cranial bone defects consist or biostable implants. They do not meet all of the demands of growing skulls. A new composite of bioactive glass S53P4 and E-glass fiber-reinforced composite, i.e. combination of bioabsorbable and biostable materials matrix was developed for the purpose. The material has been tested in preclinical and clinical studies. The implant consisted of load-bearing outer surface laminate and scaffold-like inner surface. An initial series of 20 adults with very large calvarian defects (from 50 to $150 \mathrm{~mm}$ diameter) proved that the material was both safe and well tolerated and it was incorporated in the calvarian both by bone-bonding and bone ingrowth shown on PET-CT. In the present series of 5 paediatric cases, with a defect of more than $50 \mathrm{~mm}$, age ranging from two to 14 years. Three cases were traumatic defects, one defect of infectious origin and one case of tumor ingrowth in the bone. Surgery was done 2008 to 2012, follow-up of 6 months to 4 years. Results: Implants fitted well to the defect and were easily fixated on place by help of screws and onlay lamellar margins of the implants. One case of wound edge necrosis leading to infection of the implant occurred. Other complications such as infections or allergic or toxic reactions did not occur. Conclusions: The new implant seems to offer a good tool for cranial defect repair on children, when sufficient own bone would be impossible or very demanding.

\section{1}

Fronto-orbital versus frontal remodeling in anterior plagiocephaly Laura Valentini ${ }^{1}$, Marika Furlanetto ${ }^{1}$, Paolo Cortellazzi ${ }^{1}$, Alessandra Erbetta $^{2}$, Alberto Bozzetti ${ }^{3}$, Fabio Mazzoleni ${ }^{3}$

${ }^{1}$ Department of Neurosurgery, ${ }^{2}$ Neuroradiology Unit Fondazione IRCCS Istituto Neurologico "C. Besta", Milan, Italy; ${ }^{3}$ Department of Maxillofacial Surgery, San Gerardo Hospital, Monza, Italy

Aim of craniofacial surgery is prevention or treatment of functional problems and gain of better craniofacial shape in terms of symmetry and proportion, without interfering with normal growth. Fronto-orbital cranioplasty, by reshaping and repositioning of cranial and orbital bone 
segments is the treatment of choice in growing patients, because autologous bone grafts provide lowest morbidity and don' $t$ interfere with growth. 61 patients affected by anterior plagiocephaly were treated in 2 cranio-facial departments from 2001 to 2011. Age ranged between 5 - 26 months (average 9).All were treated with a cranioplasty, 10 by a pure neurosurgical and the remaining by a combined neurosurgical-maxillofacial team. Pre and post operative evaluation included clinical and ophtalmological exam ination, $\mathrm{CT}$ and pictures standardized in the same six projection, magnification and landmarks. Long-term follow up showed: better reshaping in terms of orbital and facial symmetry by the combined approach and better stabilization of good cosmetic results by rigid fixation. There were no lack of ossification or cranial vault deformities; ocular globe position and vision were adequate except in $25 \%$ with convergent strabismus requiring orthoptic surgery. In favour of cranioplasty with fronto-orbital volet there is a more accurate bone remodelling, especially when a complex torsion is required; and a longlasting stabilization after repositioning, preventing bone resorption. Accurate planning providing overcorrection of the defect in both remodelling and repositioning may partially compensate recurrence due to phenotype. Against there are need of blood transfusions and the double step surgery to remove metal plates.

\section{2}

Change of Intracranial pressure in nineteen children with microcephalic craniosynostosis Se-Hyuck Park ${ }^{1}$, Soo Han Yoon ${ }^{2}$

Department of Neurosurgery, ${ }^{1}$ Hallym University College of Medicine, ${ }^{2}$ Ajou University School of Medicine, South Korea

Introduction: There have been many reports to date that describe the increase in intracranial pressure (ICP) in patients with craniosynostosis, but data regarding microcephalic craniosynostosis is lacking. Therefore, we report the relation between frequency of increased ICP and age in children with microcephalic craniosynostosis.

Methods: A total of 19 microcephalic craniosynostosis children underwent peri-operative ICP measurements with lumbar puncture over the past 10 years at the our hospitals, and relevant factors including age were analyzed.

Results: The mean age of the 19 microcephalic craniosynostosis study patients was $33.0 \pm 20.4$ months, the pre-operative mean ICP was $22.1 \pm$ $8.9 \mathrm{cmH}_{2} \mathrm{O}$, and the post-operative mean ICP was $15.5 \pm 4.7 \mathrm{cmH}_{2} \mathrm{O}$, demonstrating statistically significant difference $(p<0.05)$. The number of patients among the total of 19 showing increased pre-operative ICP of greater than $20 \mathrm{cmH}_{2} \mathrm{O}$ was $10(52.6 \%)$. The pattern of ICP change according to age showed gradual increase with age and reaching a peak at 42 months $(y=-0.006 \times 2+0.525 x+14.02)$, suggesting that increased ICP in craniosynostosis occurs with higher frequency than previously thought, and also peaking at 42 months of age.

Conclusion: Increased ICP is more prevalent in patients with microcephalic craniosynostosis than expected. Since the increase of ICP occurs up to 42 months of age, it is suggested that careful attention should be given to increased ICP with regular testing and examination up to 3-4 years of age with accordingly necessary and pertinent treatment.

\section{3}

Erythropoietin as strategy to decrease blood transfusion during craniosynostosis treatment: prospective study José Aloysio CostaVal, Leopoldo Mandic Furtado, Friederike Wolf Valadares

Pediatric Neurosurgery, Biocor Instituto, Vila da Serra Hospital, Nova Lima, Brazil

Introduction: The scope of this study was to evaluate the pre-operative use of erythropoietin in surgical treatment of craniosynostosis as an attempt to minimize blood transfusion.
Material and methods: We analyzed the use of erythropoietin pre-operatively in nine craniosynostosis procedures between April and November of 2012. After informed consent was obtained, human recombinant erythropoietin was given at a weekly dose of $600 \mathrm{U} / \mathrm{kg}$ until 7 days before the procedure. Treatment was repeated in case the hemoglobin did not reach $12 \mathrm{~g} / \mathrm{dl}$.

Results: During the period of the study, seven trigonocephalies, one oxycephaly and one anterior right plagiocephaly were performed. The mean age was eight months. Blood transfusion was necessary in only two cases of trigonocephaly per-operatively. There was no adverse effect to the erythropoietin.

Conclusion: Pre-operative use of erythropoietin seems to minimize blood transfusion in surgical treatment of craniosynostosis.

\section{4}

Anatomical pediatric model for craniosynostosis surgical training Giselle Coelho ${ }^{1}$, Benjamin Warf ${ }^{2}$, Marcos Lyra ${ }^{3}$, Nelci Zanon ${ }^{1}$

${ }^{1}$ Pediatric Neurosurgery Center / CENEPE, Beneficência Portuguesa Hospital, São Paulo, Brazil; ${ }^{2}$ Department of Pediatric Neurosurgery, Boston Children Hospital, Harvard Medical School, Boston, USA; ${ }^{3}$ Department of Gynecology, Federal University of Pernambuco, Pernambuco, Brazil

Introduction: Several simulators have been created as a tentative to improve the learning curve of the residents in neurosurgery and plastic surgery. Laboratory training is fundamental for acquiring familiarity with the techniques of surgery and skill in handling instruments. The aim of this study is to present a new simulator for craniosynostosis surgical training, specifically for the scaphocephaly type.

Methods: This real simulator was built with a synthetic thermo-retractile and thermo-sensible rubber which, when combined with different polymers, produces more than 30 different formulas. These formulas present textures, consistencies and mechanical resistance similar to many human tissues. The fiberglass moulds, in the shape of the skull constitute the basic structure of the craniosynostosis module trainer. It was possible to perform computadorized tomography images due to the radiopacity of this simulator and to compare the pre and post operative images.

Results: The authors present a training model to practice the biparietal remodeling used in the scaphocephaly. correction There are many possibilities to training: the skin incision; the subcutaneous and subperiostal dissection; the osteotomies, and finally the skull remodeling with absorbable microplates. The presence of superior sagital sinus can simulate emergence situations with bleeding.

Conclusion: The authors conclude that this training model can represent a fairly useful method to accustom trainees to the required surgical techniques and simulates well the steps of standard surgery for scaphocephaly. This training provides an alternative to use of human cadavers and animal models. Furthermore, it can represent the anatomical alteration precisely as well the surgical emergence situations.

\section{Congenital Spinal Disorders}

\section{5}

Intraoperative neurophysiological monitoring and mapping in tethered cord surgery

Francesco Sala ${ }^{1}$, Giovanna Squintani ${ }^{2}$, Vincenzo Tramontano ${ }^{2}$, Franco Faccioli $^{1}$, Carlo Mazza ${ }^{1}$

${ }^{1}$ Institute of Neurosurgery, Pediatric Neurosurgery, ${ }^{2}$ Division of Neurology, University Hospital, Verona, Italy

Introduction: During tethered cord surgery Intraoperative Neurophysiology (ION) averts injury to the lumbosacral nervous system by allowing a reliable identification of functional neural structures and by timely warning of an impending injury due to surgical manoeuvres. We reviewed our experience with ION in tethered cord surgery over the past decade. 
Subjects and methods: Forty-eight patients ( 36 children, 12 adults, mean age 14 years, range 0-55) underwent surgery for different lumbosacral spinal dysraphisms associated to tethered cord. We monitored cortical somatosensory evoked potentials (SEP, $n=31$ ), limb (lMEP, $n=34)$ and anal sphincter (aMEP, $n=19$ ) muscle motor evoked potentials, and the bulbocavernous reflex $(B C R, n=29)$. Neurophysiological mapping of the cauda equina was performed in all patients.

Results: Monitorable SEP, IMEP, aMEP, and BCR were obtained in $84 \%$, $97 \%, 74 \%$, and $59 \%$ of the cases respectively. In 6 patients a compound muscle action potential was evoked by stimulating tissue macroscopically considered as not functional therefore warranting further microsurgical inspection. In all patients but one SEP, MEP and BCR remained stable during surgery and ION results correlated with preserved sensory, motor and sphincter function. One patient presented IMEP and BCR drop during surgery, which correlated with transient worsening of lower limb paresis and urinary incontinence. No permanent neurological sequelae were reported.

Conclusion: Mapping techniques allow to identify and spare functional neural tissue or to cut nonfunctional structures that may contribute to cord tethering. In selected cases, changes in MEP and/or the BCR can induce a modification of the surgical strategy and, ultimately, impact on the neurological outcome.

\section{6}

\section{Clinical \& radiological progression of paediatric Chiari I} malformation: 10 year out-patient follow-up review Marcelo Santos Volpon ${ }^{1}$, Pasquale Gallo ${ }^{1}$, Guirish A. Solanki ${ }^{1,2}$ ${ }^{1}$ Department of Paediatric Neurosurgery, Birmingham Children's Hospital, Birmingham, UK; ${ }^{2}$ University of Birmingham, Birmingham, UK

Objectives: The natural history of Chiari I malformation (CMI) in children is poorly understood. The substrate responsible for progression of $\mathrm{CMI}$ and syrinx is also unclear. We report the progression of CMI over a 10 year follow-up.

\section{Design: Retrospective Review}

Subjects \& Methods: 62 (36 boys, 26 girls) children with CMI, median age of 7.8 years $(9 \mathrm{~m}-16 \mathrm{y})$, median FU 5.2 (3-10) years. Age, gender, CMI, hydrocephalus, syrinx, craniocervical junction (CCJ) CSF flow, skull base, posterior fossa measurements and symptom progression were analysed. Imaging interval dictated by clinical need. Serial MR imaging between 2002 and 2011 reviewed prior to surgery.

Results: Mean MRI interval was 3 (1-10) years. Initial CMI extent was $16.5(5-32) \mathrm{mm}$. CMI increased in $9.7 \%(6 / 62) .50 \%(3 / 6)$ required surgery. CMI regressed spontaneously in $3.2 \%(2 / 62) .12$ (19\%) had hydrocephalus, 58\% (7/12) requiring shunting. 57\% (4/7) had worsening of CMI. 48\% (30/62) had abnormal CSF flow at CCJ. 25.8\% (16/62) had syrinx. 19\% (3/16) of syrinx progressed. $6.25 \%(1 / 16)$ regressed spontaneously along with CMI hernia regression. Suboccipital or posterior calvarial surgery performed in $24 \%$ $(15 / 62)$ of symptomatic cases with a 2 year median time to surgery. $93 \%$ either had a syrinx (6/15), CSF signal loss at CCJ (6/15) or both $(2 / 15)$.

Conclusions: Hindbrain hernia remained stable in 3 out of 4 children, increased in $7 \%$, half requiring surgery. The natural history of CMI suggests that in $20 \%$ there is a rapid progression (with syrinx) requiring surgery within 2 years. Spontaneous regression is rare.

\section{7}

\section{How effective is intraoperative reduction of odontoid in Chiari I} patients with craniocervical instability?

Tina C. Loven, Harold L. Rekate, Gunes A. Aygok, Roger W. Kula, Paolo A. Bolognese, Timothy G. White

Department of Neurosurgery, Cushing Neuroscience Institute, Hofstra North Shore-Long Island Jewish School of Medicine, Manhasset, New York, USA
A significant number of Chiari I patients have craniocervical junction (CCJ) instability and anterior brainstem compression from retroflexed odontoid or from congenital weakness of the superior longitudinal band of the cruciate ligament. Management includes normalization of the occipitocervical relationship via occipitocervical fusion. The purpose of this study was to determine the efficacy of intraoperative reduction in producing normal radiographic craniocervical relationships.

Methods: Documentation of CCJ instability is controversial. We conducted a retrospective review and used three different pre- and postoperative MRI measurements; Grabb-Oakes line, Clivo-Axial angle (CXA) and a modified Grabb-Oakes line to assess the effectiveness of surgery. The data was analyzed using SPSS version 21 using independent and paired sample t-tests.

Results: There were a total of 25 patients ( 18 females, 7 males) with a mean age of 25.9 years old (age range between 9 to 52 years). All patients had diagnosis of Chiari I malformation and underwent craniocervical fusion during 2011 and 2012. The imaging findings pre-and postsurgery were as follows; the CXA increased from $128^{\circ}$ to $143^{\circ}$, the Grabb line was reduced from $9 \mathrm{~mm}$ to $6.4 \mathrm{~mm}$ and the modified Grabb from $10 \mathrm{~mm}$ to $7.3 \mathrm{~mm}$. The values between pre- and post-operative imaging findings were significantly different $(\mathrm{p}<0.005$ respectively).

Conclusions: Intraoperative reduction with craniocervical fusion was effective in normalizing radiographic relationships of craniocervical junction.

\section{8}

Re-operation after surgery of the pediatric craniovertebral junction Nobuhito Morota, Hideki Ogiwara

Division of Neurosurgery, National Medical Center for Children and Mothers, National Center for Child Health and Development, Tokyo, Japan

Introduction: Factors influencing re-operation after the pediatric craniovertebral junction (CVJ) surgery were retrospectively analyzed. Methods: Eighty five patients (aged 22 days to 15-years-old, median 1year-old) underwent 96 surgeries for CVJ lesions from May 2003 to April 2013. Data of those who had additional surgeries were analyzed in relation to age, surgical procedures and original disease.

Results: Nine patients (Three complex craniosynostosis associated with Chiari malformation type 1, two chondrodysplasia punctata, two congenital anomaly syndromes, one chordoma, one AARF) had 11 additional surgeries. There were 2 scheduled staged surgeries in 2 patients, two failed manual reduction for AARF in one, and one tumor re-growth. Six patients $(7 \%)$ underwent re-operation after insufficient or failed initial surgery (interval ranged from 1 to 86 months). Age at initial surgery for re-operated cases was as follows: 1 among 27 patients aged less than 1-year-old, 4/41 between 1 and 6-years-old, and 1/17 7-years-old and over. Regarding initial surgical procedures, two in 16 posterior fixation and 3 in 68 posterior decompressions required re-operations. There was no statistical difference among surgical procedures $(\mathrm{p}=0.22)$. Spinal instrumentation was performed in 3 patients (aged 6,10 and 15-years-old) at the time of additional surgery. Conclusions: Re-operation can be required after initial pediatric CVJ surgeries. Age factor did not necessarily influence the need of reoperation. Rather, original disease such as complex craniosysnostosis, systemic bony disease/anomalies can be regarded as a risk factor for reoperation in pediatric CVJ.

\section{9}

Timing of deformity correction in children with associated neurological abnormality requiring surgical correction Suhas Udayakumaran ${ }^{1}$, Naveen Tahasildar ${ }^{2}$, Sajesh Menon ${ }^{1}$, R. Krishnakumar $^{2}$, J. Renjith Kumar ${ }^{2}$

${ }^{1}$ Division of Pediatric Neurosurgery Department of Neurosurgery, ${ }^{2}$ Division of Spine Surgery, Department of Orthopedics, Amrita Institute of Medical Sciences, Kochi, India 
Introduction: Association of neural axis malformations with various types of scoliosis is about $2-20 \%$. Timing of scoliosis correction with respect to the neurological correction is a subject of debate.

Materials and Methods: We had a total of 9 patients (Total 591 patients of scoliosis) operated between January 2001 to January 2011 at AIMS, Kochi who required both scoliosis correction and neurosurgical procedure. We collected the demographics, Cobb's angle before procedure, surgical details, postoperative complications, cost of the procedure and long-term neurological outcome.

Results: 9 patients (below 15 years) with neurogenic scoliosis requiring both scoliosis correction and neurosurgical procedure were included. The mean age was 10.8 years. The average Cobbs angle was 59.5 degrees. 5 patients underwent foramen magnum decompression, 3 detethering and 1 intraspinal extradural tumor excision. Estimated blood loss averaged $1177 \mathrm{ml}$ (range $=700-1550 \mathrm{ml}$ ). The operative time averaged $4.4 \mathrm{hrs}$ (range $=3-5.5 \mathrm{hrs}$ ). There were no intraoperative complications. No patient had any new neurological deficit. The mean post-operative Cobb angle of main thoracic curve was 12.6 degrees. Discussion It is important to recognize the underlying neurologic factor involved in scoliosis to prevent neurologic injury. The most practiced line of management is to stage the orthopedic and neurosurgical procedure at a gap of 3-6 months. In this article, we critically evaluate our strategy of simultaneous surgical approach of rectifying the neurological issue along with scoliosis correction.

Conclusion: Simultaneous scoliosis correction along with the definitive neurosurgical procedure in neurogenic scoliosis is feasible with no added morbidity and with economic advantage.

\section{0}

Near total resection of lumbosacral lipoma. How feasible is it? Dominic N. Thompson ${ }^{1}$, Victoria Wykes ${ }^{1}$, Divyesh Desai ${ }^{2}$, Dawn Saunders ${ }^{3}$

${ }^{1}$ Department of Paediatric Neurosurgery, Great Ormond Street Hospital for Children NHS Foundation Trust, London, UK; ${ }^{2}$ Department of Paediatric Urology, Great Ormond Street Hospital for Children NHS Foundation Trust, London, UK; ${ }^{3}$ Department of Paediatric Neuroradiology, Great Ormond Street Hospital for Children NHS Foundation Trust, London, UK

Background: In 2009 Pang and colleagues described a technique of radical resection of lumbosacral lipoma, guided by neurophysiological monitoring that improved long-term outcomes ${ }^{1}$. The safety and feasibility of this technique has yet to be validated in other centres. The aim of this study was to audit an initial experience of attempted near total resection of lumbosacral lipomas.

Patients: 17 patients (median age 1.8 years) with lumbosacral lipomyelomeningocele underwent attempted radical resection of lipoma with neural placode reconstruction and expansion duraplasty. 16/17 patients were symptomatic at the time of surgery. 11 had abnormal urological profile, 8 had one or more neurological features (motor, sensory or pain) and four had foot deformity. Nerve root mapping was used to guide the resection in all cases. We aimed to perform post operative urological assessment and MRI of lumbosacral spine 3 months post surgery.

Results: Urological profile improved in 5 and was unchanged in 6 . Neurological symptoms or signs were improved in 4 and unchanged in 4. There were no instances of post operative neurological or urological deterioration. There were 4 instances of wound related morbidity, 2 pseudomeningocele requiring surgical exploration and 2 superficial infection. Post operative MRI scan showed near total resection in over half of cases.

Conclusions: Near total resection of lumbosacral lipoma guided by neurophysiological monitoring is technically feasible with low operative risk to neurological or urological function. Operative morbidity is primarily that of wound related complications.
51

Prenatal diagnosis of the myelomeningocele: Prognostic factors and its repercussion in adulthood

Artur H. G. B. Da Cunha

Hospital da Restauração, Recife, Pernambuco, Brasil

The myelomeningocele is the most common form of open spinal dysraphism, in spite of the occurrence is decreasing throughout the world, in poor countries is still occupying $20 \%$ to $30 \%$ of attention from the services of pediatric neurosurgery. In many European countries the occurrence has been decreasing the costs of interruption of pregnancy, once the diagnosis was made. The method of diagnosis must be accurate not only for identification of the pathology, as well as to guide the parents in relation to the prognosis, allowing a safe decision-making on the interruption of pregnancy or not. The author analyzes 22 patients who were born with mylomeningocele, whose diagnosis was carried out before the $20^{\text {th }}$ week of gestation and operated in the first days of life, today with age between 15 and 20 years. Patients are categorized in relation to motor impairment and intellectual achieved, and classified according to degree of independence and autonomy. There will be analyzed the effectiveness of prenatal diagnostic techniques used, characteristics of the deformity, the presence of hydrocephalus and other associated malformations and the potential to establish the prognosis of these patients with myelomeningocele. The study is still in progress and should be ready in September 2013.

\section{2}

Indications and relevance of electrophysiological measurements and IOM in pediatric neurosurgical patients

Martina Messing-Jünger, Andreas Röhrig, Jana Forkosh, Manuel Sowada Asklepios Children's Hospital, Department of Pediatric Neurosurgery, St. Augustin / Bonn, Germany

Objective: Neurophysiological measurements are of increased interest in the management of pediatric neurosurgical diagnoses. Uncertainty persists regarding age- and diagnose- related indications and the relevance of these measurements in relation to clinical findings and events, e.g. during surgery. In this study the authors try to correlate measurement findings and clinical background. Additionally limitations of the indications and interpretations were investigated.

Method: A prospective observational study was performed in order to investigate indications for clinical and intraoperative neurophysiological measurements (SSEP, MEP, VEP, EMG, nerve root stimulation). Patient data, diagnoses, findings/symptoms, surgical events and clinical course were recorded in correlation with electrophysiological technique and measurement results and time function.

Results: The preliminary results include 69 patients so far (mean age 10.6 yrs, range $0.2-48$ yrs, 5 patients $>18$ yrs, 5 patient $<1$ yr). Diagnoses were tethered cord (11), brain tumor (11), Chiari malformation (8), lipomeningocele (7), myelomeningocele (6), spinal tumor (6), syringomyelia (4), spinal deformity (3) and others (13). The positive correlation between clinical findings and intraoperative events and electrophysiological parameters was substantially high, e.g. regarding MEP results $(80-90 \%)$. Artefacts and technical problems were rare, even in the infants' group.

Conclusion: Electrophysiological measurements, either as baseline or follow-up investigation as well as during surgery (IOM) are highly reliable and technically realizable even in small children both in outpatient environments and under general anesthesia (TIVA).

\section{3}

Chiari I management: surgical roadmap

Armando Cama ${ }^{1}$, Gianluca Piatelli ${ }^{1}$, Marcello Ravegnani ${ }^{1}$, Marco Pavanello ${ }^{1}$, Alessandro Consales ${ }^{1}$, Andrea Rossi ${ }^{2}$

${ }^{1}$ Neurosurgery Unit and ${ }^{2}$ Neuroradiology Unit, G. Gaslini Children's Hospital, Genoa, Italy 
Introduction: Chiari type I malformation (CM-I) is described as caudal displacement of the cerebellar tonsils by at least $5 \mathrm{~mm}$ below the foramen magnum. Management of affected patients is controversial. We aimed to identify a roadmap for patient stratification and management.

Methods: We retrospectively analyzed a single-center cohort of 222 consecutive patients with CM-I aged between 0 and 14 years. Clinical presentation included neck pain, headaches, dysphagia, and scoliosis. All patients underwent multidisciplinary assessment including craniospinal MRI with CSF flow studies, neurophysiology, and polysomnography. Results: There were 175 (78.8\%) with a classic CM-I and $47(21.2 \%)$ with an associated cervicomedullary kink ("bulbar" CM-I). Hydrocephalus and hydrosyringomyelia were found in $12.2 \%$ and $15.8 \%$, respectively. Management included wait and see in 102 clinically silent cases (46\%) with preserved CSF flow, and surgery (with intraoperative ultrasound) in $120(54 \%)$. Surgical strategies included: i) osteo-ligamentous mini-decompression + linear dural splitting for mild clinical pictures with abnormal CSF flow; ii) decompression + duraplasty in case of cervicomedullary dysfunction or "bulbar" variants with abnormal CSF flow or hydrosyringomyelia; and iii) decompression + tonsillar resection for severe cervicomedullary dysfunctions with holocord hydrosyringomyelia and absent CSF flow. Ongoing change in surgical strategy was contemplated in case of interval clinical changes, worsening of the MRI picture or CSF flow dynamics, and presence of associated conditions such as craniosynostosis, craniocervical instability, or tethered cord.

Conclusion: We present a roadmap for management decision-making in a large pediatric CM-I cohort. Multidisciplinary management is required for optimal treatment of these patients.

\section{4}

Surgical treatment for asymptomatic filum lipoma with Anorectal/ Genito-Urinary malformations (AR/GU)

Mihoko Kato, Michihiro Kurimoto, Goro Kondo, Hirokatsu Osawa, Masato Nagasaka

Department of Neurosurgery, Aichi Children's Health and Medical Center, Aichi, Japan.

Introduction: Patients with Anorectal/Genito-Urinary malformations $(\mathrm{AR} / \mathrm{GU})$ tend to have spinal cord anomalies. However, conservative follow-up is often applied, because they cannot show neurological symptoms due to AR/GU itself and treated as an asymptomatic patient. Some pediatric surgeons suggested that patients with untreated tethered cord were more likely to have poor bowel function after anorectal malformation repair. We performed untethering for filum lipoma patients with $\mathrm{AR}$ GU, even patients did not show neurological symptoms. In this study, we tried to evaluate our strategy.

Methods: A total of 26 consecutive patients with filum lipoma were retrospectively analyzed.

Results: Pre-operative MRI was performed for all patients. Tethered Cord (TC) was detected 6/8 (75\%) patients with AR/GU and 8/18 (44\%) patients without AR/GU. Among patients with AR/GU, 3/3 (100\%) of neurologically symptomatic patients and $3 / 5(60 \%)$ of asymptomatic patients showed TC. Among patients without AR/GU, 6/9 (67\%) of neurologically symptomatic patients and only $2 / 9(22 \%)$ of asymptomatic patients showed TC. $(\mathrm{P}=0.058)$ No major operative complications occurred. Six of 12 symptomatic patients showed neurological improvement. The other 6 patients were unchanged. All of asymptomatic patients stayed asymptomatic after surgery.

Conclusions: As previous reports, patients with AR/GU often showed TC in MRI. Focusing on asymptomatic patients, TC detected more often in patients with AR/GU than without it. Neurological symptoms of AR/GU patients could be difficult to find and finally be selected conservative follow-up. It might deprive them of therapeutic chance. We believe that patients with $\mathrm{AR} / \mathrm{GU}$, even if asymptomatic, should be surgically treated.
55

Outcome in 32 cases of tethered cords in adults - is intervention justified?

Sandip Chatterjee

Department of Neurosurgery, Park Clinic, Kolkata, India

Introduction: Adults rarely present with tethered cord syndrome, and this review examines whether it is justifiable to perform surgical intervention in this group.

Methods: Between 2002 and 2011, we performed surgical intervention in 32 adults with tethered cord syndrome. The age range varied from 22 years to 65years. 26 presented with pain, 20 had motor deficits, and 18 had sensory deficits, whereas sphincter dysfunction was present in 17 . Three of the patients had undergone surgery for meningomyelocele as infants. 6 patients had major spinal deformity. All patients underwent digital radiographs and MR scans of the whole spine.

Results: The mean follow-up was 3.2years (range 1 year - 8.5 years). 21 out of 26 patients presenting with pain had their VAS scores improve significantly, 14 had motor deficits which improved. However sensory deficits got better in only 7 out of 18 patients, and sphincter improvement was documented (via urodynamics studies and bladder ultrasound) in only 4 out of 17 patients. In 15 cases surgery was performed under neurophysiological monitoring as this was not available to patients earlier in the series, and overall improvement was documented in 11 of these patients compared to 7 of the remaining 13 patients. Surgery for spinal deformity was performed in 6 patients and deformity correction as well as pain reduction was achieved in all.

Conclusion: Surgery for tethered cords in adults leads to significant reduction of pain by untethering, and in those with spinal deformity by correction of the same. Improvement in sensory changes occurred in far less patients, and those with sphincter problems did not show significant improvement at all. Neurophysiological monitoring certainly has improved our results, although our numbers are too small to draw meaningful inferences.

\section{6}

Atlantoaxial rotatory subluxations in Indian children: A review Subhas Konar, Sandip Chatterjee

Department of Neurosurgery, Park Clinic, Kolkata, India

Aim: To study the clinical presentation and management of rotatory subluxations at the atlanto-axial joint. Introduction: C1-C2 rotatory subluxations are rare. Early recognition and prompt reduction is a key to successful treatment.

Methods: We retrospectively reviewed 18 cases (Male:12, Female:6) of paediatric atlantoaxial subluxation from January 2001 till January 2012. All these cases were evaluated clinicoradiologically by means of Digital Xrays, Computer Tomography in positions- neutral, torticollis position, after maximum correction and Magnetic Resonance Imaging scans. The patients were evaluated for etiology, associated trauma, treatment undertaken, outcome.

Results: The average time from trauma to treatment ranged from 3 days to 6 months. One patient had trivial trauma and presented with traumatic torticolis and only one presented after 6 months as the original trauma was missed due to polytrauma. The remaining presented after falls, tuberculosis of C1-C2 or without antecedent trauma. In three cases dynamic lateral flexion scans were done as missed in regular imaging. After preliminary skull traction to all cases, twelve cases reduced and out of these three were treated with halo vest fixation for three months and the remaining eight with Philadelphia collars alone. Four were treated with operative methods, one was operated by $\mathrm{C} 1-\mathrm{C} 2$ transarticular fixation, two by direct reduction and C1-C2 joint arthrodesis , and one was operated by occipito cervical fixation. Mean Follow-up was 37 months(range, 12-64 months).

Conclusion: Patients presenting early with rotatory subluxation can be treated only conservatively by traction if they present early; the rest 
require operative fixation by the posterior approach if closed reduction cannot be achieved.

\section{7}

Long-term outcome in congenital spinal dysraphisms: a single center observational study of adult cohorts

Deepak Gupta, Sachin Borkar, Ashish Suri, S. S. Kale, Ashok Kumar Mahapatra

Department of Neurosurgery, All India institute of Medical Sciences, New Delhi, India

Introduction: Children born with spinal dysraphism anomalies often found to have significant neurological and urological sequelae and often succumb to life threatening end stage renal disease secondary to neurogenic bladder.

Materials: This is a single center study of 23 cases (age $>16$ years, 15 males, 8 females) of spinal dysraphism treated at our hospital from 20082012. Pain was noted in 14 cases (61\%), sensorimotor symptoms in 11 (47\%) cases, bladder/bowel involvement noted in $12(52 \%)$ cases and prophylactic surgery before scoliosis correction for spinal dysraphism was noted in $5(21 \%)$ cases, one patient was incidentally detected and operated. Radiologically, besides low lying tethered cord in all cases, split cord malformations were noted in 3 cases, conus lipoma in 7 cases and neuroenteric cyst/dermal sinus in one case each. Previous history of detethering was noted in one case while 2 cases were previously operated for meningomyelocele at birth.

Observations: Mean age for patients with bladder involvement was 27.3 years and conus lipoma was noted in $6 / 12$ cases with pre-existing neurogenic bladder. End stage kidney disease was noted in 2 patients (both females).

Outcome: Improvement in motor symptoms was noted in 5/11 cases while 6 remained unchanged. Urinary symptoms improved in $2 / 12$ cases only. Conclusions: Decreasing incidence of spinal dysraphism patients presenting at our hospital has been noted in past one decade. Close follow-up and appropriate intervention have improved outcome in such cases but renal deterioration remains a risk in majority.

\section{8}

Successful treatment of a recurrent aneurysmal bone cyst affecting the cervical spine with a novel antibody therapy in an 11 years old boy

Christian Hagemann ${ }^{1}$, Gabriele Escherich ${ }^{2}$, Reinhard Schneppenheim ${ }^{2}$, Philip O. S. Kunkel ${ }^{1}$

${ }^{1}$ Department of Pediatric Neurosurgery, Altona Children's Hospital, Hamburg, Germany; ${ }^{2}$ Department of Pediatric Hematology and Oncology, University Hospital Hamburg-Eppendorf, Hamburg, Germany

Introduction: Aneurysmal bone cysts (ABCs) represent locally expansive and destructive bone lesions exhibiting osteoclast markers. Standard treatment is complete curettage and cavity filling. In spinal cases when total resection is not possible adjuvant treatment options include embolization and radiation therapy. In the cervical spine of children these options are limited. Denosumab, a human monoclonal antibody inhibits osteoclast function by blocking receptor activator of nuclear factor kappa B ligand (RANKL). Therapeutic efficacy was previously demonstrated in giant cell tumors of the bone, which display similar immunohistochemical patterns compared to $\mathrm{ABCs}$, thus suggesting a potential therapeutic effect in $\mathrm{ABCs}$.

Methods: We report here our experience with the first patient treated with denosumab for a recurrent spinal aneurysmal bone cyst. 8 months after intralesional resection and ventro-dorsal reconstruction of the spinal column an 11 years old boy showed extensive recurrence of an $\mathrm{ABC}$ affecting the entire $\mathrm{C} 5$ vertebra. Arterial embolization failed due to the absence of appropriate feeding vessels. After interdisciplinary tumor board discussion and informed consent given by the family we started denosumab therapy as an individualized treatment.

Results: MRI follow-up studies at 3, 6 and 12 months after the start of denosumab administration showed progressive regression of the tumor and consolidation of the bone substance. Severe side effects were not observed. Conclusions: Given validation in larger cohorts and longer follow-up denosumab represents a potential treatment option in non-resectable cases of spinal ABCs.

\section{9}

Intradural release of filum terminalis in occult tethered cord syndrome (OCTS): does it work?

Flavio Giordano ${ }^{1}$, Maria Taverna ${ }^{2}$, Stefano Ricci ${ }^{1}$, Barbara Spacca ${ }^{1}$, Luigi Sardo ${ }^{1}$, Alfredo Danti ${ }^{2}$, Lorenzo Genitori ${ }^{1}$

${ }^{1}$ Department of Neurosurgery, ${ }^{2}$ Department of Urology, "Anna Meyer" Pediatric Hospital, Firenze, Italy

Occult Tethered Cord Syndrome (OTCS) is characterized by urological complaints (i.e. hyperactive bladder) and sometimes leg and back pain without clear-cut radiological signs of tethered cord. Intradural section of filum terminalis is a surgical option though its real efficacy is still to be confirmed. Aim of this study is the analysis of surgical outcomes in a series of eleven patients with OTCS. Since 2009 eleven patients fulfilling all diagnostic criteria underwent intradural release of filum terminalis. There were 7 males and 4 males; mean age was 11 years (range 4-19 years). Average duration of symptoms were 31.3 months (range 12-84 mos). Clinical findings were: urinary (8) and fecal incontince (1), recurrent urinary tract infections (2). All patients received a complete urological workup to rule out other etiology. Medical treatment with oxybutinin was tried before surgery. After 10.8 months mean follow-up (range 6-30 mos) nine patients referred a subjective improvement of urological complaints; two subjects did not feel any variation. None of the patients worsened after surgery. Post-operative urodynamic testing showed objective improvement of bladder function in two patients. In one child there was a complete normalization of bladder activity. Surgical morbidity occurred in one girl because of CSF leak with pseudomenyngocele that needed a surgical repair. These results confirm intradural section of filum terminalis as a possible treatment for OCTS after failure of conservative therapies. However, larger series and longer follow-up are needed to definitely validate this surgical option.

\section{0}

Spine shortening in recurrent tethered cord syndrome

Yusuf Ersahin, Yildiz Aygenli

Section of Neurosurgery, Kent Hospital, Izmir, Turkey

Surgery of recurrent tethered cord syndrome (TCS) is not always easy and untethering of the spinal cord can be impossible. Vertebral column shortening (VCS) has been described in the management of TCS in adults. Two patients with recurrent TCS in which VCS had been performed will be presented.

Case 1. A 17-year-old girl presented with severe neuropathic low-back pain radiating to both legs. She was operated on for a tight filum terminale and had undergone two more surgeries for recurrent TCS. MRI revealed an adhesive arachnoiditis. Medical treatment and spinal cord stimulation did not alleviate the pain. Posterior VCS (T11-12) was performed and a $1.5 \mathrm{~cm}$-spine shortening was achieved. Neuropathic pain was relieved completely after surgery. She is doing well 17 months postoperatively. Case 2. A 44-year-old man with lipomyelomeningocele who had undergone 4 spinal surgeries presented with severe neuropathic pain in lower limbs for the past year. He had paraparesis and urinary incontinence. Posterior VCS (T10-11) was performed and an $2 \mathrm{~cm}$-spine shortening was achieved. Neuropathic pain was relieved completely after surgery. He has 
no further complaint 16 months following surgery. In 1995 Kokubun described a case report of spinal osteotomy for VCS in a patient with a low-lying conus medullaris. Grande et al used a cadaveric model and found that shortening the vertebral column by $15-25 \mathrm{~mm}$ significantly reduces tension on the spinal cord, nerve roots, and filum terminale. VCS by subtraction osteotomy provides an alternative surgical treatment for recurrent TCS.

\section{1}

Juvenile amyotrophy of the distal upper extremity (Hirayama disease), a disease of transition to adulthood: Case series \& surgical management

Laura Grazia Valentini ${ }^{1}$, Giuseppe Piscosquito ${ }^{2}$, Luisa Chiapparini ${ }^{3}$, Donatella Milani ${ }^{4}$, Cecilia Casali ${ }^{1}$, Marika Furlanetto ${ }^{1}$

${ }^{1}$ Department of Neurosurgery, ${ }^{4}$ Department of Pediatric, ${ }^{2}$ Adult Neurology, ${ }^{3}$ Neuroradiology Unit Fondazione IRCCS Istituto Neurologico " $\mathrm{C}$. Besta”, Milan, Italy

Hirayama firstly reported (1959) a disorder of the juvenile age (11-25 years), characterized by unilateral or asymmetric slowly progressive amyotrophy of the distal upper extremity, so named Hirayama Disease (HD). After the initial progressive course was reported a spontaneous arrest. Other definitions are: Monomyelic Amyotrophy or Cervical Flexion Myelopathy (CFM), proposed by Kikuchi based on the possible etiology: a compression of the cervical spinal cord during neck flexion. Etiology and treatments are still debeated: conservative prolonged collar therapy, cervical arthrodesis, cervical duroplasty - laminoplasty and muscolotendineous transfer were proposed in few published series. The clinical and radiographic characteristics of 14 patients affected by CFM are reported: mean onset age was $16(14-25)$ years, with a male prevalence $(12 / 14) ; 8$ were operated (expansive duraplasty-suspension by laminotomy), 6 were managed conservatively. Neutral and fully flexed neck MRI and neurophysiology were performed along Fup. Dynamic MRI showed posterior dural loss of attachment and cervical cord flattening. 1/8 operated cases experienced transient worsening, 1/8 progressed despite surgery, while in the remaining 6 deterioraton stopped; All presented at surgery venous engorgement and spinal cord tendency to herniate by dural opening; $1 / 8$ had also arachnoid adhesions. In our series clinical suspicion of HD was confirmed by dynamicMRI, so reinforcing the neck flexionMRI for proper diagnosis. Early diagnosis is a milestone because clinical outcome may be strikingly improved by early surgery. Especially in rapidly progressive disease surgery may be considered awaiting for spontaneous arrest, whilst a mild HD course suggests conservative approach.

\section{Craniofacial Surgery II}

\section{2}

Mandibular and facial lower third development in adult patients with previously treated unicoronal synostosis

Luca Massimi $^{1}$, Gianpiero Tamburrini ${ }^{1}$, Giulio Gasparini ${ }^{2}$, Tito M. Marianetti $^{2}$, Alessandro Moro ${ }^{2}$, G. Saponaro ${ }^{2}$, Massimo Caldarelli ${ }^{1}$, Sandro Pelo ${ }^{2}$, Concezio Di Rocco ${ }^{1}$

${ }^{1}$ Pediatric Neurosurgery, A. Gemelli Hospital, Catholic University Medical School, Rome, Italy; ${ }^{2}$ Maxillo Facial Surgery Unit, Complesso Integrato Columbus, Catholic University Medical School, Rome, Italy

Purpose: Maxillary and mandibular alterations in patients affected by anterior synostotic plagiocephaly usually receive a scarce attention if compared with skull and orbital changes. The aim of this study is to assess the facial development in adult patients who underwent the correction of their unicoronal synostosis during the first year of life. Methods: Twelve adult patients were selected (mean age: 25.5 years). All of them were early treated by a neurosurgical fronto-orbital correction and completed the maxillo-mandibular growth at the time of the evaluation. Maxillary and mandibular metric measurements were performed on high resolution, thin slices CT scans; all images were reconstructed into 3D models which were used to perform the metrical assessments.

Results: Significant differences were found between the two sides, all measured distances being shorter in the synostotic side than in the non-synostotic one, with exception of the vertical dimension of the mandibular ramus. Such discrepancies were more evident in patients with more severe plagiocephaly (type IIb and III of the Di Rocco's classification). The mandibular alterations were characterized by lower jaw hypoplasia on the side of the affected suture and anterior displacement of the glenoid fossa; these two entities compensated each other uncompletely, leading to a rotation of the mandibula towards the synostotic side.

Conclusion: Although classified as "simple" synostosis, anterior plagiocephaly significantly affects the development of the whole splanchnocranium (other than the neurocranium) with an evident alteration of skeletal, along with facial, eurythmy.

\section{3}

Synostotic posterior plagiocephaly: clinical, radiological and neuropsychological features at diagnosis and the long-term outcome

Gianpiero Tamburrini ${ }^{1}$, Alba Scerrati ${ }^{1}$, Daniela Chieffo ${ }^{2}$, Massimo Caldarelli ${ }^{1}$, Luca Massimi ${ }^{1}$, Concezio Di Rocco ${ }^{1}$

${ }^{1}$ Pediatric Neurosurgery, Institute of Neurosurgery, Catholic University, Rome, Italy; ${ }^{2}$ Pediatric Neurorehabilitation Unit, Bambino Gesù Hospital, Palidoro, Rome, Italy

Introduction: The correct diagnosis, treatment and long-term prognosis of posterior synostotic plagiocephaly is debated; only limited series and short-term outcomes are reported in the literature.

Patients and methods: We retrospectively reviewed all the cases of synostotic posterior plagiocephaly operated on at our Institution in the last 10 years time. The diagnosis was confirmed by a preoperative 3D CT scan in all but 3 cases. All children underwent a comprehensive preoperative neuropsychological evaluation. Surgical technique consisted in occipital expansion and bone remodeling through the barrel-stave technique with fixation by silk wires or resorbable plates.

Results: Fifteen patients were collected. Differently from previous series, posterior homolateral displacement of the ear did not represent a reliable diagnostic parameter, being present in only 8/15 cases. CT scans invariably documented the synostosis of the lambdoid suture, a compensatory controlateral parietal bossing and a homolateral condylar deviation. The most frequent neuropsychological deficit was represented by visuomotor attention deficits (11/15 cases). Satisfactory correction of the posterior asymmetry was obtained in all cases (mean follow-up : 5.6 years); no change was observed in hemifacial, condilar and skull base asymmetry. Discussion and conclusions: Synostotic posterior plagiocephaly might constitute a more complex disease than what is commonly thought. CT scan should be adviced in all doubtful cases to confirm the diagnosis. Selective cognitive deficits are present preoperatively in a significant percentage of cases. Surgical treatment is only able to correct the posterior asymmetry; it cannot influence the associated skull base deformities.

\section{4}

Morphological characterization of the foramen magnum in syndromic \& multisuture synostosis

Pasquale Gallo $^{1}$, Anne L. S. Elserius ${ }^{1}$, Pete Noons ${ }^{1}$, D Rodrigues ${ }^{1}$, Guirish A. Solanki ${ }^{1,2}$

${ }^{1}$ Department of Paediatric Neurosurgery, Birmingham Children's Hospital, ${ }^{2}$ University of Birmingham, Birmingham, UK 
Introduction: Syndromic craniosynostosis (SCS) accounts for $10 \%$ of all craniosynostosis and harbours the more severe forms of craniocephalic disproportion including within the foramen magnum.

Aims \& Objectives: Describe foramen magnum (FM) morphology in different types of SCS.

Patients \& Methods: 81 (50 boys, 31 girls) children with SCS underwent MRI morphometry of the FM, including FM Surface Area (FMSA), FM anterior-posterior diameter(FMAP) and FM transverse diameter (FMTD). Chiari1 (CM1) \& Syringomyelia, when present, were measured.

Results: In Apert's (21/81) FMSA range was $308-1037 \mathrm{~mm}^{2}$, FMAP ranged between 18.3-37.4mm. In Crouzon's (26/81) FMSA ranged 261.2-949.6mm ${ }^{2}$ and FMAP $14.8-46.8 \mathrm{~mm}$. In Multisuture (15/81) the FMSA ranged 259-641 $\mathrm{mm}^{2}$ and FMAP 16.7-31.4mm. In Pfeiffer's(6/81) the FMSA ranged $253.8-675.9 \mathrm{~mm}^{2}$ and FMAP $16.2-39.2 \mathrm{~mm}$. Overall FMSA ranged from 253.8-1040 $\mathrm{mm}^{2}$. Mean FMSA was 523 (without $\mathrm{CM} 1=488$, with $\mathrm{CM} 1=579) \mathrm{mm}^{2}$. FMAP ranged from 14.8-48.5 mm. Mean FMAP was 27.1 (without $\mathrm{CM} 1=25.9$, with $\mathrm{CM} 1=28.5$ ) $\mathrm{mm}$. FMSA is greater with Chiari than without, However when syrinx was present, maximum FMSA was 10 to $35 \%$ smaller in all syndromic groups.

Conclusion: We identify for the first time the relative proportions of the foramen magnum in syndromic CS. Pfeiffer's have the smallest and Muenke have the largest FMSA. Crouzon's have the highest incidence of Chiari and syringomyelia. FMSA is smaller in controls and SCS children without Chiari compared to SCS with Chiari I alone. In the presence of syringomyelia FMSA is smaller than with Chiari alone. These findings highlight the crucial importance of the foramen magnum morphology in the aetiology of syringomyelia and development of Chiari Malformation.

\section{5}

\section{D-CT findings of occipital sutures in children younger than 2 years} of age

Ai Muroi ${ }^{1,2}$, Takao Enomoto ${ }^{3}$, Satoshi Ihara ${ }^{1}$, Akira Matsumura ${ }^{1}$

${ }^{1}$ Division of Pediatric Neurosurgery, Ibaraki Children's Hospital, Ibaraki, Japan; ${ }^{2}$ Department of Neurosurgery, Tsukuba University Hospital, Ibaraki, Japan; ${ }^{3}$ Department of Neurosurgery, Tsukuba Central Hospital, Ibaraki, Japan

Introduction: The anatomy of occipital bone in younger children is complex due to a lot of variations of an ossification pattern. The aim of this study is to clarify the normal variation of occipital suture in 3D-CT and the diagnostic pitfalls.

Methods: We reviewed 223 head 3D-CT underwent between 2008 and 2011 retrospectively. Variations of occipital suture, skull deformity and medical history of patients younger than 2 years of age were investigated. Results: 102 patients had head injury and 30 had some congenital anomaly. 3D-CT showed wormian bone in 68 patients, mendosal suture in 74 , median fissure in 47 , interparietal suture in 16 , interparietal bone in 30. 25 patients had skull fractures.

Discussions: 3D-CT was useful to demonstrate various patterns of occipital sutures. Variation seen in this study was similar to that in previous anatomical studies. However, a careful evaluation using other diagnostic tools including skull X-rays and CT axial images was needed to distinguish a fracture from a normal suture.

Conclusions: 3D-CT of skull of younger children showed a lot of variations of occipital suture, which helped a better understanding of normal development of occipital bones and a differential diagnosis of fractures in occipital area.

\section{6}

Spring-assisted posterior cranioplasty compaired to conventional posterior cranioplasty

Marie-Lise C. van Veelen ${ }^{1}$, Tim de Jong ${ }^{2}$, Irene M. Mathijssen ${ }^{2}$

${ }^{1}$ Department of pediatric neurosurgery, Erasmus University Medical Centre Rotterdam; ${ }^{2}$ Department of Plastic and Reconstructive Surgery, Erasmus University Medical Centre
Introduction: Posterior cranioplasty is indicated as first intervention in syndromic craniosynostosis without severe exorbitism or severe obstructive sleep apnea and as second intervention in case of recurrent raised intracranial pressure. The extend of conventional posterior cranioplasty is limited by difficulties to close the skin. Spring-assisted posterior cranioplasty does not have this disadvantage. In this study springassisted posterior cranioplasty is compared to conventional posterior cranioplasty in syndromic craniosynsostis.

Methods: 15 patients underwent spring-assisted cranioplasty between 2009-2012, these patients were compared to 16 patients who underwent conventional posterior cranioplasty between $2006-2009$. The technique of spring-assisted posterior cranioplasty consists of craniotomy lines placed parallel to and behind the coronal sutures and just above the transverse sinus, leaving a small hinge over the torcula. After a limited dissection of the bone from the dura 4 springs $(1.02 \mathrm{~mm}$ medical steel wire, $6 \mathrm{~cm}$ width, 6 Newton distractive force), are placed in the vertical osteotomy lines.

Results: Mean age at surgery, mean duration of surgery, mean blood loss and complications were comparable between both techniques. Postoperative head circumference as well as the anterior-posterior length of the skull increased significantly better after spring-assisted than after conventional posterior cranioplasty $(\mathrm{p}=0.029$ resp $\mathrm{p}=0.028$ ). Preoperative papillary edema resolved after springassisted cranioplasty in 7 out of 7 cases, after conventional posterior cranioplasty only 5 out of 7 cases of preoperative papillary edema resolved.

Conclusions: Spring-assisted posterior cranioplasty is as save as conventional posterior cranioplasty and results in a larger head circumference and anterior-posterior length.

\section{7}

Major clinical features of synostotic occipital plagiocephaly; Mechanisms of cranial deformations

Hamilton Matushita ${ }^{1}$, Nivaldo Alonso ${ }^{2}$, Maria Rita Passos Bueno ${ }^{3}$, Daniel Dante Cardeal $^{1}$, Fernanda Andrade ${ }^{1}$

${ }^{1}$ Division of Pediatric Neurosurgery, Department of Neurosurgery, São Paulo University; ${ }^{2}$ Division of CranioFacial Surgery, Department of Plastic Surgery, São Paulo University; ${ }^{3}$ Department of Genetic, São Paulo University, São Paulo, Brazil

Introduction: The clinical diagnosis of most common monosutural craniosynostosis is easily set, based on the stereotype of deformities and knowledge of the mechanisms of cranial deformations. However, synostosis of unilateral lambdoid suture, probably due to its lower incidence and similarity with other nonsynostotic deformities affecting the posterior portion of the skull, makes its clinical diagnosis not easily done.

Objectives: The aim of this study is to evaluate the most easily clinical characteristics to be recognized in the synostotic occipital plagiocephaly.

Material and Methods: This study consisted of clinical evaluation of 8 patients with synostotic occipital plagiocephaly, whose diagnosis was further corroborated by computed tomography.

Results: We observed: Trapezoidal contour of the skull in top view in 6/8 (75\%); Parallelogram contour of the skull in posterior view on $2 / 8$ (25\%); Bulging of ipsilateral mastoid process in $8 / 8(100 \%)$; "Edge effect" of ipsilateral lambdoid suture in $8 / 8(100 \%)$; Horizontal unlevelling of the bi-mastoid line in 3/8 (37.5\%); Anterior and inferior deviation of the ear in $8 / 8(100 \%)$; "Dumbo" ears in $8 / 8$ $(100 \%)$; Frontal bulging in $2 / 8(25 \%)$ and Contralateral parietal bossing in $6 / 8(75 \%)$.

Conclusion: The most important clinical features to the clinical diagnosis of synostotic occipital plagiocephaly were bulging of the ispilateral mastoid process, ipsilateral "edge effect" of the lambdoid suture, anterior and inferior deviation of the ear, and "Dumbo" ear. 
68

A 30-year experience in treatment of saggital craniosynostosis Jivko K. Surchev ${ }^{1}$, Kiril D. Georgiev ${ }^{1}$, Yavor P. Enchev², Asen V. Bussarsky $^{1}$

${ }^{1}$ Department of Neurosurgery, Medical University, Sofia, Bulgaria; ${ }^{2}$ Department of Neurosurgery, Medical University, Varna, Bulgaria

Background: The surgical treatment of the saggital craniosynostosis aims to correct the deformation to esthetically acceptable skull shape and in many cases to decompress the brain.

Objects: The aim of this study was to evaluate retrospectively and to compare the efficacy of 2 groups operative technique in patients with scaphocephaly.

Material and methods: For the period 1982 - 2011 year, at the Department of Neurosurgery, Medical University Sofia were evaluated and treated 123 children with saggital craniosynostosis or $50.45 \%$ of all synostoses, treated during that period. 84 of the patients were male and 39 - female, as the median age was 11.7 months. 115 of the cases were operated with 2 groups operative techniques: Group 1 - bilateral parasagittal linear craniectomies with a wide of $1.5-2 \mathrm{~cm}$. (in 7 cases) and extensive linear craniectomies (bilateral bone flaps) (in 21 children) - totally 28 patients; Group 2 - subtotal calvarectomy and remodeling of the cranial vault (in 87 patients). Quantitative assessments of the operative results, based on the preoperative and postoperative (1 year after the procedure) measurements of the cephalic index (cranial width/cranial length $x$ 100) and statistical analysis were performed in all patients.

Results: For Group 1, the mean preoperative cephalic index was 66.0 and the postoperative index -71.2 (mean improvement of 5.2), which was bellow the each standarts. For Group 2, the mean preoperative cephalic index was 65.56 and the mean postoperative index 76.79 (mean improvement of 11.23) - in the age normative values.

Conclusion: Based on the comparison of the cephalic indexes at the two groups, we considered the subtotal calvarectomy and calvarial remodeling as operative technique of choice of scaphocephaly.

69

Bilateral lambdoid and sagittal synostosis (BLSS) - "Mercedez Benz" Syndrome

Hamilton Matushita ${ }^{1}$, Nivaldo Alonso ${ }^{2}$, Maria Rita Passos Bueno ${ }^{3}$, Daniel Dante Cardeal ${ }^{1}$, Fernanda Andrade ${ }^{1}$

${ }^{1}$ Division of Pediatric Neurosurgery, Department of Neurosurgery, São Paulo University; ${ }^{2}$ Division of CranioFacial Surgery, Department of Plastic Surgery, São Paulo University; ${ }^{3}$ Department of Genetic, São Paulo University, São Paulo, Brazil

Introduction: A peculiar type of craniosynostosis consists of a combination of bilateral lambdoid and sagittal synostosis. The triradiate ridging of the synostotic sutures resembles the emblem of "Mercedez Benz". Few of them have complete clinical and radiological documentation, the best surgical technique is still controversial.

Objectives: The authors present a series of 6 children with multisutural craniosynostosis with involvement of bilateral lambdoid and sagittal sutures, in order help define its clinical features and treatment.

Material and Methods: All patients were examined for general clinical and neurologic aspects, morphometric measurement, and computed tomographic and magnetic resonance imaging of the cranium.

Results: The age ranged from 9 to 72 months (mean $=28 \mathrm{~m}$ ), there was predominance for gender $M / F=4 / 2$, and there was no specific ethnic distribution. The major cranial deformities observed were brachycephaly (mean cephalic index $=0.89$ ), increased frontal bossing 4/6 $(66.6 \%$ ), increased occipital concavity in $3 / 6(50 \%)$. Neuropsychological delay was observed in 3/6 (50\%). Other malformations observed: Chiari I Malformation in 2/6 (33.3\%), agenesis of the corpus callosum in sixth (16.6\%), ear malformation in one (16.6\%), and hydrocephaly was not observed.
Surgical treatment aimed at the expansion and remodeling of the parietal, occipital, and suboccipital regions, with no surgical complications.

Conclusion: Our results showed etiological heterogeneity of this rare type of craniosynostosis and demonstrated a varied phenotype, requiring for a correct diagnosis, assistants diagnostic tests. Surgical treatment with remodeling of the posterior cranium showed effective morphological correction.

\section{0}

Total skull reshaping of craniosynostosis with distraction using multi-directional cranial distraction osteogenesis system

Yasushi Sugawara $^{1}$, Ataru Sunaga ${ }^{1}$, Kunio Miyazaki ${ }^{1}$, Yoko Katsuragi ${ }^{1}$, Hideaki Kamochi ${ }^{1}$, Akira Gomi ${ }^{2}$

${ }^{1}$ Department of Plastic Surgery, ${ }^{2}$ Department of Pediatric Neurosurgery Jichi Medical University, Tochigi, Japan

Background: Total cranial reconstruction for sever deformity of multiple suture synostosis is still difficult procedure because of poor rigidity of reconstructed cranium with fixation materials, soft-tissue resistance against expansion, and multiple bone defect which are the cause of postoperative relapse. We have developed a new method of distraction osteogenesis for craniosynostosis with pan-cranial deformity that uses a new multi-directional cranial distraction osteogenesis system (MCDO system). The MCDO system consists of a disposable clear plastic frame, anchor pins and extension rods for fixing the frame on the skull, traction pins for pulling the bone pieces up, a distractor fixed on the frame, and plastic flanges for protecting the holes.

Methods: From 2008 to 2012, 7 selected patients with syndromic and multiple suture craniosynostosis were treated with MCDO system. Mean patient age was 11 months. Follow-up was 7 to 49 months (median: 13 months).

Results: The postoperative course was uneventful in all cases. The mean postoperative hospital stay was 12 days. The phase of activation was 8 to 14 days (mean, 10.5 days) and the consolidation period was 21 to 40 days (mean, 28 days). The morphological results of all cases were satisfactory. Conclusion: The MCDO system allows the contour of any kind of skull deformities as desired under the concept of distraction osteogenesis. The distraction method with MCDO system offered numerous advantages over not only the distraction method with unidirectional internal devices but also conventional cranioplasty for the patient with multiple suture synostosis.

\section{1}

The needle in a haystack - unilateral lambdoid craniosynostosis Tilmann Schweitzer, Hartmut Collmann, Beatrice Jager, Jürgen Krauss Section of Pediatric Neurosurgery, Department of Neurosurgery, University of Würzburg, Germany

Introduction: In the literature the incidence of premature unilateral lambdoid synostosis is reported to be 0.003 . Over the past 2 years we noticed a rise in the number of infants with unilateral lambdoid synostosis, referred to our outpatient clinic.

Methods: In two years we saw 7 infants with a premature unilateral lambdoid synostosis. 3 of these were misdiagnosed as positional plagiocephaly, while 3 came with unclear diagnosis. In only one case the tentative diagnosis of lambdoid synostosis was already set. We report on these cases and the findings of the recommended MRI. The ongoing routine follow-up includes repeated clinical examination, ophthalmoscopic exclusion of papilledema and skull $\mathrm{x}$-rays for convolutional markings. Results: A Chiari malformation was apparent on MRI in 4 cases, interestingly with 2 cases of progressive Chiari malformation. 2 children had no Chiari malformation, while in one infant it is still unknown. The Chiari cases showed unilateral downward displacement of the ipsilateral tonsil with only mild crowding of the Foramen magnum and absence of 
ventricular enlargement, therefore Chiari decompression was not indicated. One case was remodeled because of marked deformity.

Conclusion: The higher number of true lambdoid synostosis presented to our outpatient clinic might be due to the increased awareness towards skull asymmetries because of positional plagiocephaly and molding therapy. Correct diagnosis of this rare entity is important because of the necessity for extended diagnostic workup and sometimes for operative treatment.

\section{2}

\section{Skull base fibrous dysplasia: When to operate}

Graciela Noemi Zuccaro, Romina Alejandra Argañaraz

Department of Neurosurgery, Hospital Nacional de Pediatría "Juan P. Garrahan", Buenos Aires, Argentina

Fibrous dysplasia is an abnormal fibroseous process of bone of unknown etiology. When this disorder affects the skull base it may result in encroachment of structures, such as the orbit, paranasal sinuses, and skull base foramina leading to blindness, deafness, nasal obstruction, etc. Between 1990 and 2012, we treated 35 children with skull base fibrous dysplasia, 19 of whom, 14 female/ 5 male with a mean age of 12 years, underwent surgery. The other 16 were closely controlled. The patients were operated on using combined craniofacial and transpalatine approaches. In none of them the lesion was totally removed. Unroofing of the encroached optic canal and orbital expansion were performed in cases of progressive visual loss. Children who had already developed amaurosis were not selected for surgery. In cases of nasal obstruction, we also used the frontoethmoidal approach. Function improved in all patients and in some total recovery was achieved.

Conclusions: We believe the best management of skull base fibrous dysplasia is radical excision, however, impossibility to achieve this goal is the rule in extensive disease. In such cases the goal of surgery is to correct the cosmetic deformity, if it is significant and prevent complications such as visual loss. Radiotherapy should not be used because of the risk of inducing malignancy. With modern craniofacial techniques good functional and cosmetic results are obtained even in cases of extensive bone removal.

\section{Hydrocephalus}

\section{3}

Neural stem and progenitor cells collected from the hydrocephalic CSF form neurospheres that exhibit the same junction pathology as the disrupted ventricular zone

Montserrat Guerra ${ }^{1}$, Nicole Lichtin ${ }^{1}$, M. Clara Jara ${ }^{1}$, Roberto González ${ }^{1}$, Karin Vío ${ }^{1}$, César González ${ }^{1}$, Eduardo Ortega ${ }^{2}$, Jaime Jaque ${ }^{2}$, Sara Rodríguez $^{1}$, Alexander Ortloff ${ }^{3}$, Paula Salazar ${ }^{1}$, Pat McAllister ${ }^{4}$, Conrad Johanson $^{5}$, Esteban M. Rodríguez ${ }^{1}$

${ }^{1}$ Instituto de Anatomía, Histología y Patología and ${ }^{2}$ Instituto de Neurociencias Clínicas Facultad de Medicina, Universidad Austral de Chile, Valdivia, Chile; ${ }^{3}$ Escuela de Medicina Veterinaria, Universidad Católica de Temuco, Chile; ${ }^{4}$ Department of Neurosurgery, Division of Pediatric Neurosurgery, University of Utah, Salt Lake City, Utah, USA; ${ }^{5}$ Department of Neurosurgery, Alpert Medical School at Brown University, Providence, RI, USA

The ventricular zone (VZ) contains neural stem cells (NSCs) while the subventricular zone (SVZ) harbors neural precursor cells (NPCs). Evidence obtained from human fetuses and mutant animals with hydrocephalus indicates that a cell junction pathology in NSCs and NPCs leads to disruption of the VZ and SVZ. To determine the fate of the disrupted cells, this study analyzed the proliferation and differentiation of NSCs and NPCs obtained from hydrocephalic CSF (hyCSF). At postnatal day 1 (PN1), cells obtained from the VZ/SVZ of non-hydrocephalic HTx rats or the CSF of hydrocephalic HTx rats were cultured for six days (6DIV) and exposed to $\mathrm{BrdU}$ on the last day. They were processed for immunofluorescence using antibodies for proliferation (BrdU), differentiation (nestin, beta-3-tubulin, GFAP), functional plasma membrane proteins (AQP4, caveolin 1) and adherent junctions (N-cadherin). NSCs and NPCs were present in the hyCSF; they proliferated and formed small and large neurospheres with distinct immunocytochemical differences that progressively disassembled and formed new neurospheres. Neurosphere cells showed an abnormal expression of $\mathrm{N}$-cadherin, i.e. junction proteins accumulated in the cytoplasm. In contrast, neurospheres from the non-hydrocephalic rats grew to form compact neurospheres that did not disassemble with $\mathrm{N}$-cadherin located normally at the plasma membrane. These findings suggest that hyCSF contains NSCs and NPCs that form neurospheres with the same junctional pathology as the hydrocephalic VS/SVZ in vivo. Thus, neurospheres obtained from patients and animal models may be used to investigate the cellular and molecular alterations underlying congenital hydrocephalus.

\section{4}

The obstructive nature of pediatric hydrocephalus - A comparison of high resolution MRI and conventional MRI imaging Miriam Seiß $^{1}$, Thomas Naegele ${ }^{2}$, Martin U. Schuhmann ${ }^{1}$

${ }^{1}$ Section of Pediatric Neurosurgery, Department of Neurosurgery and ${ }^{2}$ Department of Neuroradiology, Eberhard Karls University Hospital, Tuebingen, Germany

Introduction: Modern hydrodynamic theories of hydrocephalus discuss CSF flow obstruction or disturbance of pulsatility as etiological factor for the development of hydrocephalus. Standard-MRI images are designed to detect brain tissue but not CSF space abnormalities. High-resolution (HR) MRI is a powerful tool to detect obstructive pathology in the CSF compartment. This study compares HR-T2 to standard T2 sequences regarding the detection rate of CSF pathway obstructions.

Methods: We examined MRI scans of 56 children with hydrocephalus diagnosed within first year of life. Median age at time of MRI was 27,5 month, range 0 month -17 y. Standard multiplanar $3 \mathrm{~mm}$ T2-sequences were assessed and compared to multiplanar $1 \mathrm{~mm}$ true-fisp $(\mathrm{n}=49)$, CISS $(n=2)$ and HR-T2 $(N=5)$ sequences obtained at the same time. A senior neuroradiology (TN) and pediatric-neurosurgery consultant (MUS) rated 112 imaging series blinded and randomized.

Results: TN found obstructions in 51 childrens in standard and in 56 in HRMRI $(p<0.05)$. MUS found obstructions in 40 children in standard and in 56 in HR-MRI $(p<0.05)$ The total number of obstructions sites in standardT2 was 188 and 107 compared to 383 and 362 in HR-MRI according to TN and MUS respectively $(\mathrm{p}<0.005)$. Considerably more obstructions were detected at aqueduct, foramen of Luschkae and basal cisterns.

Conclusion: HR-MRI classified all pediatric hydrocephalus as obstructive in nature, with a 2-3 fold higher detection rate. Especially outflow obstruction from 4th ventricle and basal cistern obstruction could be visualized. HR-MRI should become standard in diagnostic hydrocephalus imaging.

75

Effects of postural changes on intracranial pressure: Preliminary results

Amer Hadi, Morten Andresen, Marianne Juhler

Department of Neurosurgery, Section 2092, Rigshospitalet, Copenhagen, Denmark

Background: Changes in intracranial pressure (ICP) based on body posture, have clinical implications for shunt selection and configuration. Previous studies have focused on lumbar cerebrospinal fluid pressure and ICP changes due to head elevation of the bed. Consequently, we currently lack proper reference values for ICP in different body postures. Objectives: To quantify the effects of postural changes on ICP by continuous cerebral measurement. 
Methods: Patients requiring invasive ICP monitoring were included prospectively, and performed postural changes between supine, right lateral, right lateral lumbar puncture position, sitting on a chair, sitting on a chair lumbar puncture position, and standing. Each position was maintained for 10 minutes. We noted factors such as shunt, drainage, blood pressure, height, weight, spine length, distance from the lateral ventricles to right atrium, and abdominal circumference.

Results: 11 monitoring sessions were performed ( 5 patients $<18$ years). In the supine position, mean of medians was $5.5(5.5) \mathrm{mmHg}(\mathrm{SD})$. The ICP changed to 9.3 (5.6) $\mathrm{mmHg}$ and -5.7 (6.8) $\mathrm{mmHg}$ for lateral recumbent and standing positions, respectively. The mean difference in ICP between supine and standing was $11.2 \mathrm{mmHg}(\mathrm{p}<0.001)$. Changing body posture from sitting to standing was non-significant $(\mathrm{p}=0.638)$. Lumbar puncture position during lateral recumbent and sitting increased ICP significantly, $\mathrm{p}=0.045$ and $\mathrm{p}<0.001$

Conclusions: Characterization of postural changes in ICP is necessary in order to arrive at a correct diagnosis, as well as optimize shunt design, and treatment of shunt patients. In our preliminary data, the additional factors noted did not affect the size of ICP variation.

\section{6}

High function shunt dependent children becoming functional adults are facing important psychological challenges

Liana Beni-Adani

Dana Children's Hospital (TASMC) and Tel-Aviv University, Tel-Aviv, Israel

Introduction: Pediatric hydrocephalus presents psychological challenges with adulthood mainly in patients with high functional profile. Body image, fear of sudden shunt-malfunction at work or as parents are some of the meaningful issues patients are faced with.

Methods: Three high function women with shunted hydrocephalus (ages 24-26) and 1 male (22y) followed closely since childhood were interviewed. All 4 were living full life, 3 women were married and 2 had children by the time of interview. The young man was studying after a period of volunteering. All had experienced at least 1 shunt revision in the past, and $3 / 4$ had mild-moderate motor deficits. The questions were related to their main difficulties during adulthood in relation to hydrocephalus and shunt.

Results: Hydrocephalus was due to infantile meningitis in the male, and associated with MMC (1), huge retrocerebellar-cyst (1) and post-IVH prematurity-related HCP (1) in the female patients. The main difficulties expressed were not neurological/motor deficits but psychological. The women stressed their past fears about difficulty to get married, and later the fear from shunt complications during pregnancy or delivery. Parenthood was very important despite the fears expressed. Body image was less important compared to second decade of their life. All four expressed the importance of being functional individuals at work or as parents and stressed the importance of family support.

Conclusions: Family support seems to be the most important factor to enable shunted adults overcome their major fears while trying to fully function as parents or contributing individuals in society.

77

Controversies in pediatric hydrocephalus

Dieter Class, Raimund Firsching

University Department of Neurosurgery, Magdeburg, Germany

Objective: There are many controversies concerning diagnostic and surgical treatment of hydrocephalus. We tried a survey by sending a questionnaire to colleagues in numerous countries known to be engaged in the treatment of hydrocephalus.

Methods: The questionnaires included details on diagnostic and surgical procedures in premature newborns and in childhood up to the age of 18 years.
Results: 117 completed questionnaires from 12 countries in Europe, South America, the USA and Israel were anonymously returned for evaluation. $17 \%$ relied on ultrasound and magnetic resonance imaging (MRI) in all premature newborns only. 12\% performed computerized tomography $(\mathrm{CT})$ in all patients. Among the surgical procedures implantation of a reservoir was employed by a majority. The minimum body weight acceptable for the placement of a ventriculo-peritoneal shunt ranged from $400 \mathrm{~g}$ to $3500 \mathrm{~g}$. The length of the peritoneal catheter ranged from 10 to $110 \mathrm{~cm}$. During follow-up of the growing child a planned revision to prolong the peritoneal catheter was performed by a minority. The majority implanted adjustable and gravitational valves, but fixed pressure systems also played a major role.

Conclusion: Obviously there is a plethora of views on fundamental principles of the treatment of hydrocephalus. Many questions remain unanswered. An international data bank could possibly help to identify the more feasible and beneficial measures. It could be particularly helpful to developing countries facing many children to be treated and often without highly sophisticated technology.

\section{8}

Long-term consequences of untreated "compensated" hydrocephalus in children are not necessarily benign Chris Parks ${ }^{1}$, Mohammed Al-Jumaily ${ }^{2}$, Michael D. Jenkinson ${ }^{3}$, Peter Murphy ${ }^{4}$, Conor Mallucci ${ }^{1}$

${ }^{1}$ Department of Neurosurgery, Alder Hey Children's Hospital, Liverpool, UK; ${ }^{2}$ Department of Neurosurgery, University Hospital of North Staffs, Stoke-on-Trent, UK; ${ }^{3}$ Department of Neurosurgery, The Walton Centre, Liverpool, UK; ${ }^{4}$ Department of Neuropsychology, Walton Centre, Liverpool, UK

Introduction: Longstanding overt ventriculomegally in adults (LOVA) is described as chronic hydrocephalus starting in infancy, evolving slowly and presenting with a variety of clinical disturbances during adulthood. This study measured neuropsychological outcome in a group of patients presenting with "compensated hydrocephalus" in order to assess the longterm consequences.

Methods: A retrospective cohort of 20 patients treated by the senior author for LOVA was identified. All fulfilled the diagnostic criteria including severe ventriculomegally, macrocephally and/or neuroradiological evidence of expanded or destroyed sella turcica. All patients had aqueduct stenosis and were treated at decompensation with endoscopic third ventriculoscopy. Patients were followed up, interviewed and asked to complete a questionnaire exploring presenting and post-surgical symptoms, childhood symptoms, education and academic achievements, occupation and satisfaction with treatment and its outcome. Validated neuropsychological and behavioural assessments were also performed. Results: Repeatable battery for the assessment of neuropsychological status (RBANS) gave a mean score for this group of 71 compared to 100 in the general population. Only 3 patients finished higher education but $11(55 \%)$ were in employment at the time of assessment. $80 \%$ of patients (16) had symptoms that were retrospectively missed during childhood related to hydrocephalus. Walking imbalance was most common $(45 \%)$ followed by headaches, memory problems and cognitive problems. Symptoms improved in most following treatment.

Discussion: It is likely that patients with "compensated" hydrocephalus never fulfill their neuropsychological potential and have a significant risk of even late decompensation as adults.

\section{9}

Conclusions after 941 hydrocephalus valve-tests within 22 years Aschoff Alfred

Heidelberg, Germany

Introduction: After 22 years our valve laboratory was closed. 
Methods: 941 hydrocephalus valves were tested: 90 designs, 385 new and 521 probes explanted after max. 21 years. 212 proximal, 24 distal slit valves (17/10 designs), 106 diaphragm (20), 160 simple, 197 adjustable and 206 gravitational-(g) ball valves (9/10/11 versions) and 36 other valves ( 8 designs). We measured conform ISO 7191:1989, made. 179 long-term-tests (121 for 360-500 days) and up to 36 subtests dedicated to reflux, susceptibility to external fluid pressure, vectorial forces, flection, temperature, protein, pulsations, pumping, sterilizations, MRI (1.5-4.0T, adjustable-) and body movements (g-valves)

Results: Every third valve only met producers spezifications. $24 \%$ deviated $\pm 21-50 \mathrm{mmH}_{2} \mathrm{O}$, each $15 \% \pm 51-80$ respect $\pm 81-135$ $\mathrm{mmH}_{2} \mathrm{O} .13 \%$ failed up to $1446 \mathrm{mmH}_{2} \mathrm{O}$. Ball-valves were superior with $61 \%$ good and $21 \%$ fair results. After 365-500 days drifted $89 \%$. $32 \%$ of the valves were not reflux-resistant, $29 \%$ influenced by external fluid pressure, $50 \%$ susceptible to vectorial forces and $56 \%$ to flections. In upright $81 \%$ overdrained $(>100 \mathrm{ml} / \mathrm{h}), 9 \%$ showed insufficient flow. Adjustable showed similar flow as fixed valves in similar ranges; Many had a lack of robustness and in longterm adjustment-problems. The 17 adjustable valves require 35 different adjustment-tools leading increasing confusion. ASDs have in lab and clinics problems with safety, Orbis-Sigma with accuracy. The best physiological functions showed g-valves.

Conclusions: 1 . The majority of valves showed still deficits in accuracy, long-term-stability, safety and physiological flow-rates. 2. Intelligent shunts are no longer a "Mission Impossible".

\section{0}

Hydrocephalic cerebrospinal fluid is a friendly host for neural stem cell transplantation

Pat McAllister ${ }^{1}$, César González ${ }^{2}$, M. Clara Jara ${ }^{2}$, Karin Vío ${ }^{2}$, Roberto González $^{2}$, Montserrat Guerra ${ }^{2}$, Eduardo Ortega ${ }^{3}$, Jaime Jaque ${ }^{3}$, Sara Rodríguez $^{2}$, Paula Salazar ${ }^{2}$, Conrad Johanson ${ }^{4}$, Esteban M. Rodríguez ${ }^{2}$ ${ }^{1}$ Department of Neurosurgery, Division of Pediatric Neurosurgery, University of Utah, Utah, USA; ${ }^{2}$ Instituto de Anatomía, Histología y Patología and ${ }^{3}$ Instituto de Neurociencias Clínicas Facultad de Medicina, Universidad Austral de Chile, Valdivia, Chile; ${ }^{4}$ Department of Neurosurgery, Alpert Medical School at Brown University, Providence, RI, USA

It is now understood that hydrocephalus is not only a disorder of CSF dynamics but also a brain disorder and that surgery does not resolve most aspects of the disease. Based on the evidence that the common history of foetal-onset hydrocephalus and abnormal neurogenesis starts with the disruption of the neural stem (NSCs) and neural precursor (NPCs) cells forming the ventricular and the subventricular zones, we sought to explore strategies that might help repair such a loss, including neural stem cell grafting. NSCs and NPCs from the telencephalon of PN1 and PN7 non-hydrocephalic HTx rats were cultured to obtain neurospheres. After 7 days in vitro (DIV) they were grown for 2 weeks in a culture medium free of growth factors and containing normal (nCSF) or hydrocephalic (hyCSF) CSF. Neurospheres from non-hydrocephalic rats were grafted into the lateral ventricle of PN7 hydrocephalic rats. Neurospheres cultured in nCSF and hyCSF disaggregated and differentiated into neurons and glia; the length of neurites was similar under both conditions. hyCSF+culture medium maintained for 1 DIV resulted in the appearance of small neurospheres. During the $2^{\text {nd }}$ DIV neurospheres differentiated into cells with long processes. When pure hyCSF was incubated at $37^{\circ} \mathrm{C}$ large neurospheres developed. The grafting of neurospheres into the CSF of hydrocephalic rats is in progress and early results are promising. In conclusion, the CSF of normal and hydrocephalic animals promotes the differentiation of NSCs/NPCs in neurospheres into neurons and astrocytes, and hyCSF appears as a friendly medium to host grafted neurospheres.
81

Efficacy of non-contrast-enhanced time-spatial labeling inversion pulse (time-SLIP) method in treatment of congenital central nervous system anomalies

Mario Suzuki ${ }^{1}$, Takaoki Kimura ${ }^{2}$, Akihide Kondo ${ }^{1}$, Kazuaki Shimoji ${ }^{1}$, Masakazu Miyajima ${ }^{1}$, Shinya Yamada ${ }^{3}$, Hajime Arai ${ }^{1}$

${ }^{1}$ The Department of Neurosurgery, Juntendo University, School of Medicine, Tokyo, Japan; ${ }^{2}$ The Department of Neurosurgery, Charité - Universitätsmedizin Berlin, Berlin, Germany; ${ }^{3}$ The Department of Neurosurgery, Toshiba Rinkan Hospital, Sagamihara, Japan

Background: Recently, non-contrast Time Spatial Labeling Inversion Pulse (time-SLIP) method, a new sequence of magnetic resonance (MR) imaging has been developed to investigate the movement of cerebrospinal fluid (CSF). We have applied this imaging method to evaluate surgical indications and postoperative assessment in congenital anomalies relating in CSF circulation disorders.

Methods: We reviewed patients who had been treated for CSF circulation disorder and evaluate with time-SLIP between 2010 and 2012.

Results: During this period, 10 hydrocephalus, 3 Chari type 1 malformations, 3 arachnoid cysts, and 1 craniosynostosis were treated. In hydrocephalus, we had focused who those underwent endoscopic third ventriclostomy (ETV). It is well known that T2 heavy weighted image is important to evaluate the patency of the stoma. Time SLIP method could add a dynamic image of the movement of the CSF going back and forth through the stoma. In Chiari type 1 malformation, it was possible to visualize the improved CSF movement around the cisterna magna. In arachnoid cysts, this method was able to visualize the CSF movement between the cistern and the cyst through the fenestration performed with the neuroendoscope.

Conclusions: In each cases time SLIP method was able to visualize dynamic flow of the CSF and provided important information. This method is a less invasive examination which can repeatedly use without contrast enhancement media. This Time-SLIP method is an efficient way to evaluate children with CSF circulation disorders.

\section{2}

\section{Computational fluid dynamics of ventricular catheters} for the treatment of hydrocephalus: a 3-D analysis Marcelo Galarza ${ }^{1}$, Angel Gimenez Pastor ${ }^{2}$, Olga Pellicer Porcar ${ }^{3}$, Jose Valero Cuadra ${ }^{2}$, Amigo Garcia Jose Maria ${ }^{2}$

${ }^{1}$ Servicio de Neurocirugía, Hospital Universitario Virgen de la Arrixaca, Murcia, España; ${ }^{2}$ Departamento de Estadística, Matemática e Informática, Universidad Miguel Hernández, Elche, España; ${ }^{3}$ Departamento de Psicología de la Salud, Universidad Miguel Hernández, Elche, España

Objectives: A landmark study addressed the problem of fluid characteristics in ventricular catheters by using a 2-D simulation program of computational fluid dynamics (CFD). Our objective was to study ventricular catheter designs by using a 3-D CFD models. This will produce a visual image of the flow field and results will be integrated to evaluate mass flow rates. Materials: The general procedure for the development of a CFD model involves incorporating the physical dimensions of the system to be studied into a virtual wire-frame model. The fluid properties and motion are calculated at each of these grid points. After grid generation, flow field boundary conditions are applied and the fluid's thermodynamic and transport properties are included.

Results: New designs of ventricular catheter that incorporates varying hole pattern distributions and size dimensions of the ventricular catheter were conceived and were tested by using a 3-D CFD study. The expected variations in the geometrical features significantly changed the entering mass flow rate distribution along the ventricular catheter.

Conclusions: Ventricular catheters with variable hole diameters along the catheter tip will be allowed fluid to enter the catheter 
more uniformly along its length, thereby reducing the probability of its becoming occluded.

\section{3}

Etiology and outcome of pediatric hydrocephalus in one institute: A ten-year report

Chenkai Ma, Baochen Wang, Qifeng Li, Jie Ma

Department of Pediatric Neurosurgery, Xinhua Hospital, Shanghai Jiao

Tong University, School of Medicine, Shanghai, China

Introduction: Hydrocephalus is a worldwide common condition, with varied etiology. Despite of the novel technique and management of hydrocephalus, the outcome of operation for hydrocephalus varies from different region.

Methods: We retrospectively extracted and assessed the clinical and radiological data of all patients diagnosed as hydrocephalus during 2003 to 2012 from medical records. The reoperation rates and efficiencies of treatment for hydrocephalus were compared in terms of different age and surgical approach.

Results: Four hundred and fifty-three patients met our inclusion criteria, $44.9 \%$ of which accompanied with malformation. Second to the malformation, children with hydrocephalus resulted from intracranial tumors, at $18.1 \%$. VPS and etV were the main therapeutic operation for hydrocephalus, which helped patients recover from hydrocephalus effectively. The reoperation rate of VPS and etV were $35.25 \%(98 / 278)$ and $10.79 \%(15 / 139)$, respectively. The Odds Ratio (OR) is 5.05 and there is statistical significant $(\mathrm{p}<0.0001)$. The main reason for second surgery after VPS was infection and overdrainage.

Conclusion: The outcome of etV for hydrocephalus is much better than that of VPS. Patients underwent etV receive markedly less reoperation and complications than that who receive VPS.

\section{4}

Efficacy of ventricular access device versus ventriculosubgaleal shunt for the post-hemorrhagic hydrocephalus in very low birth weight preterm infants

Shunsuke $\mathrm{Ichi}^{1}$, Yasuo Iwasaki ${ }^{3}$, Ichiro Suzuki ${ }^{1}$, Atsushi Nakao ${ }^{2}$, Tadashi Kawakami $^{2}$

${ }^{1}$ Department of Neurosurgery, ${ }^{2}$ Department of Neonatology, Japanese Red Cross Medical Center, Tokyo, Japan; ${ }^{3}$ Department of Neurosurgery, International University of Health and Welfare Mita Hospital, Tokyo, Japan

Introduction: The surgical treatment of post-hemorrhagic hydrocephalus $(\mathrm{PHH})$ in very low birth weight (VLBW) preterm infant is still challenging. The efficacy of two different devices, ventricular access device (VAD) and ventriculosubgaleal shunt (VSGS) were compared retrospectively.

Methods: Eighteen infants with PHH, weighing less than $1500 \mathrm{~g}$ at birth and initial surgery, were treated with VAD in 1994-2003 versus VSGS in 2004-1013. The VSGS system was designed with small CSF reservoir that allowed tap of CSF if needed.

Results: The VADs and initial VSGSs were placed at age of $39 \pm 18$ days and $38 \pm 26$ days respectively. All ten infants treated with VAD required daily CSF tap at volume of $12 \pm 3.7 \mathrm{ml}$ for $81 \pm 54$ days while two out of eight infants treated with VSGS required several times of tap just before VP shunt replacement. Revision of VSGS prior to safe replacement to VP shunt was needed for two infants both at 28th day after initial VSGS because of limited survival of shunt i.e. limited subgaleal absorption of CSF. Infection of device was observed in two out of ten infants with VAD at $7^{\text {th }}$ and $45^{\text {th }}$ post operative day respectively. All eight infants with VSGS had no device infection before VP shunt replacement. The switching to VP shunts was at the age of $117 \pm 49$ days for VAD group and $104 \pm 40$ days for VSGS group. No need for VP shunt was seen in an infant of VAD group.

Conclusions: The VSGS is safe and physiological way of treatment as a temporary control of ICP for PHH of VLBW preterm infants.

\section{5}

Adjustable Medos-valves: Shunt-survival after 18-22 years not better as with simple valves

Aschoff Alfred ${ }^{1}$, Benesch Christoph ${ }^{2}$, Piotrowicz Agnieszka ${ }^{3}$

${ }^{1}$ Heidelberg; ${ }^{2}$ Department of Neurosurgery, Aschaffenburg; ${ }^{3}$ Department of Neurology, Lübeck, Germany

Introduction: Real long-term results, $>10$ years, after shunts are rare; reports of adjustable valves $>10$ years are missing. In 1990 we implanted the first serial Medos-valves (Medos-P) worldwide after in-vitro-tests.

Methods: Between 6/1990 and 12/2010 we observed 670 patients with 862 adjustable Medos-P in a prospective registry including documentation of all adjustment and complications. 601 patients were primary implanted in our hospital, 59 externally. 130 Medos-P were tested in laboratory, of them 120 explanted specimen. The decisions to select a Medos-P were free for the surgeons; most prefered it in "difficult cases" such as children and iNPH. Since 1991 in selected cases the Medos-P were supplemented with ASDs, since 1993 with gravitational valves, which became common later on. The data of 110 patients $(55 \%$ adult, $45 \%$ children) operated 1990-94 were reevaluated and supplemented with actual contacts (at least via phone), to get data on life quality (Karnowskyindex), school success, carriere etc..

Results: The valve-independent complications counted $30 \%$ in distal catheters (preferably peritoneal). Caused by many preterm babies and the extreme long observation there were $20 \%$ infections. Related to valverelated problems $10 \%$ showed valve-dysfunctions and $7.3 \%$ chronic subdurals. The Kaplan-Meier-survival in adult and children counted $58 \%$ after 60 and $56 \%$ after 120 months; after 160 months survived $50 \%$, in children $35 \%$ only.

Conclusions: In preferebly "difficult patients" the Medos-P showed a shunt-survival not better to simple valves. The theoretical advantages were counterbalanced by valve-failures and subdurals.

\section{6}

Intraoperative ultrasound guidance improves the accuracy of ventricular catheter placement and the 6 month shunt survival rate

Llewellyn C. Padayachy ${ }^{1}$, Celeste De Jager ${ }^{2}$, Anthony A. Figaji ${ }^{1}$, Graham Fieggen ${ }^{1}$

${ }^{1}$ Pediatric Neurosurgery Unit, Red Cross Children's War Memorial Hospital, University of Cape Town, Cape Town, South Africa; ${ }^{2}$ Epidemiology and Biostatistics Unit, University of Cape Town, Cape Town, South Africa

Introduction: Shunt failure rates have remained high despite significant technical advances. Proximal catheter occlusion remains an important cause of shunt dysfunction. Accurate ventricular catheter placement, therefore, remains an attractive goal in the quest to improve overall shunt survival rates. Methods: We present an analysis of prospectively collected data on ventricular catheter placement for 47 patients with an initial diagnosis of hydrocephalus. Real-time intra-operative ultrasound (IOUS) guidance was used for ventricular catheter placement in twenty one patients, and the freehand placement technique in twenty six patients. The position of the catheter tip relative to the ipsilateral foramen of Monro was calculated using a Euclidean formula and also subjectively assessed using radiological criteria to grade (grades 1-3) the catheter position on the postoperative CT scan. The overall shunt failure rate in both groups were compared at 1,3 and 6 months. 
Results: The mean catheter accuracy in the IOUS group was significantly better than in the IOUS group than in the freehand group $(14.10 \mathrm{~mm} \pm$ 10.82 vs $23.34 \pm 10.36, p<0.002,95 \%$ confidence interval [3.99-16.5]). The overall shunt survival rate at 6 months in the IOUS group was $73.3 \% \%$, vs $69.3 \%(\mathrm{p}=0.23)$ in the freehand group. Proximal catheter obstruction, however accounted for only $11.1 \%$ of shunt failure in the IOUS group vs $19.2 \%(\mathrm{p}<0.05)$ in the freehand placement group. There were no complications related to the use of IOUS, with negligible time added to surgery.

Conclusion: IOUS is a safe and effective neuronavigation modality which improves the accuracy of ventricular catheter placement and proximal catheter survival rate at 6 months.

\section{7}

\section{Shunt weaning test: method to assess possibility of shunt removal} for shunt dependent hydrocephalus

Masahiro Nonaka ${ }^{1}$, Shin Nakajima ${ }^{1}$, Mami Yamasaki ${ }^{2}$

${ }^{1}$ Department of Neurosurgery, Osaka National Hospital, Osaka, Japan; ${ }^{2}$ Department of Pediatric Neurosurgery, Aijinkai Takatsuki Hospital, Takatsuki, Japan

Shunt trouble, such as shunt blockage and shunt infections are extremely traumatic experience for both patients and their caretakers. They live their life in a constant state of alert looking for signs of the next failure. Thus, we believe freedom from a shunt dependent state is ultimate goal. Although patients who had a shunt malfunction have chance for shunt removal by endoscopic procedures, there is no established method for patients with working shunts who wish to have their shunts removed. Since 2009, we attempted to remove shunts for 29 patients with congenital hydrocephalus. 16 patients received shunt removal at the time of shunt malfunction. And the rest of 13 cases with working shunt, we applied shunt weaning test to investigate possibility of shunt removal. Shunt weaning tests were performed as follow: After externalization of the distal end of existing shunt catheter, opening pressure were gradually elevated up until patients become symptomatic, or amount of drained fluid become zero. Five out of 13 cases remained asymptomatic during test, and their shunt was removed without any additional procedures. 8 cases became symptomatic, and then underwent endoscopic third ventriculostomy (ETV). However, 3 cases after etV, shunts were required again. Overall shunt free rate was $76.9 \%$. Our study support that shunt weaning test is an effective method to screen patients who just can remove shunts without other procedures, and select candidates suitable for etVs.

\section{8}

Treatment strategy for occlusive hydrocephalus due to posterior fossa tumors - early tumor resection is effective

Florian Volz ${ }^{1}$, Rudolf Korinthenberg ${ }^{2}$, Vera van Velthoven ${ }^{1}$

${ }^{1}$ Department of Neurosurgery, ${ }^{2}$ Department of Pediatrics and Adolescent Medicine, University Medical Centre, Freiburg, Germany

Object: Children with posterior fossa tumors often present with symptoms of elevated intracranial pressure due to occlusive hydrocephalus. Treatment options are endoscopic third ventriculostomy (ETV), extraventricular drainage (EVD), VP-Shunt or early resection of the causing tumor. There is still no consensus if CSF diversion prior to the tumor operation reduces the risk of persisting hydrocephalus. We report that early surgical removal of the tumor alone is effective for treatment of hydrocephalus, and that a routine use of etV is not justified.

Methods: We retrospectively analyzed our series of children with posterior fossa tumors.

Results: 50 patients between 5 months and 18 years with infratentorial tumors were analysed. All patients presented with symptoms of increased intracranial pressure, $33(73 \%)$ had radiological signs of hydrocephalus. 5 patients $(10 \%)$ received preoperative CSF diversion, because tumor surgery was not considered treatment of choice on admission. 2 with etV, 2 with EVD, 1 with etV and VP-shunt, in all cases tumor surgery was performed subsequently. Following our standard approach we performed early tumor resection without preoperative CSF diversion in 45 patients. 40 of them (89\%) had decreasing clinical and radiological signs of hydrocephalus and no need for further operative intervention. 5 patients $(11 \%)$ had persistence of hydrocephalus on malresorptive grounds requiring VP-shunt implantation.

Conclusions: We conclude that early tumor resection is sufficient treatment for hydrocephalus due to posterior fossa tumors. Considering the low rate of persisting -but not obstructive- hydrocephalus after tumor resection, a routine use of preoperative etV is not justified.

\section{9}

The adjustable gravitational valve proSA to overcome CSF overdrainage in young and adolescent hydrocephalus patients Sebastian Antes ${ }^{1}$, Wolfgang Wagner ${ }^{2}$, Joachim Oertel ${ }^{1}$, Christoph A. Tschan $^{1}$

${ }^{1}$ Department of Neurosurgery, Saarland University Medical Center and Saarland University Faculty of Medicine, Homburg/Saar, Germany; ${ }^{2}$ Department of Neurosurgery, Section of Pediatric Neurosurgery, University Hospital of Mainz, Mainz, Germany

Introduction: CSF overdrainage is still one of the most frequent complications in shunt-treated hydrocephalus. Particularly with regard to young and adolescent patients, treatment of manifest overdrainage is complicated by several hardly influenceable factors as increasing height and mobility. A new gravitational valve allowing postoperative adjustments of the pressure level seems to be a promising solution to meet the requirements. Methods: Sixty-four shunt-treated young hydrocephalus patients aged $1.8-41.4$ years $(15.5 \pm 10.5$ years $)$ suffering from clinical and/or radiological manifested overdrainage underwent shunt revision surgery with the implantation of the new proSA valve. Clinical outcome as well as the valve chamber function after a one-year follow-up period was retrospectively analyzed.

Results; All patients reported a significant improvement of their clinical condition. 91\% described a complete resolution in symptoms related to overdrainage. Preoperatively, more than $85 \%$ of the valve chambers showed no or very slow re-filling with intracranial CSF whereas more than $90 \%$ exhibited a normal valve chamber function after one year of proSA treatment. During the follow-up period, a total of 136 valve adjustments were necessary, mostly to increase the pressure level due to further overdrainage.

Conclusions: The possibility of postoperative adjustability in opening pressure level is the decisive advantage of the new gravitational valve. Excellent outcome in adolescent and adult hydrocephalus patients is attributed to the new valve technology.

\section{0}

Inclusion of gravity assisted units in first time pediatric hydrocephalus shunts - Influence on shunt outcome Thayane Fritz ${ }^{1}$, Artemisia Dimostheni ${ }^{1}$, Karin Haas-Lude ${ }^{2}$, Martin U. Schuhmann ${ }^{1}$

${ }^{1}$ Section of Pediatric Neurosurgery, Department of Neurosurgery, ${ }^{2}$ Department of Neuropediatrics, Eberhard Karls University Hospital, Tuebingen, Germany

Introduction: The addition of a gravity assisted unit (GAU) at the time of first shunt implantation in pediatric hydrocephalus is thought to prevent overdrainage from the very beginning. However, influence of GAUs on the shunt revision rate is unclear. We compared shunt survival data of two cohorts of children with first-time implanted adjustable shunts with and without GAU.

Methods: Between 1/2006 and 08/2010 82 kids underwent first-time shunt implantation, starting 2008 GAUs were added in increasing frequency. 31 kids received Codman Medos programmable-valve (without 
GAU group A) and 33 Miethke proGAV valve (including GAU, group B). A retrospective data analysis was performed.

Results: Mean follow-up for group A was 40.7 months, for Group B 30.3 months. In Group A 18/31 (58\%) underwent further shunt related surgery, in Group B 15/33 (45\%). Valve related reasons for revision (overdrainage-underdrainage-valve occlusion) were found in 8-1-4 patients, respectively in Group A (72\%) and 0-2-2 respectively in Group B $(27 \%)$. Non-valve-dependent reasons for revision (catheter related-infection-wound healing problems) were found in 5-0-1 patients in Group A and 6-1-5 patients in Group B, respectively.

Conclusions: The addition of a GAU to an adjustable valve system at firsttime shunt implantation does not seem to negatively influence the rate of shunt surgery during follow-up. In this study it significantly lessened, however, the chance of overdrainage or other valve-related interventions.

\section{Hydrocephalus and Neuroendoscopy}

\section{1}

Clinical features and outcomes of endoscopic third ventriculostomy for pediatric patients with non-communicating hydrocephalus

Chenkai Ma, Feng Jiang, Xiaoguang He, Jie Ma

Department of Pediatric Neurosurgery, Xinhua Hospital, Shanghai Jiao Tong University, School of Medicine

Introduction: Hydrocephalus is a quite common disease from varied etiology in children, with high morbidity. Endoscopic Third Ventriculostomy (ETV) has gradually become the recommended approach for noncommunicating hydrocephalus.

Methods: One hundred and thirty-nine children were underwent etV for non-communicating hydrocephalus during 2006 to 2012 in our center. Clinical features, total hospital expenditure and failure of etV were collected retrospectively. Patients with hydrocephalus second to malignant tumors were excluded of the cost analysis.

Results: Of the included patients, male and female are 90 and 49, respectively. The mean age of the patients with hydrocephalus is $2.44 \pm 2.81$ years. In terms of etiology, majority of the patients accompany with other malformation (including cysts and myelomeningocele), accounting for $36.69 \%$. Second to the malformation, patients who were examined with hydrocephalus by radiological images occasionally are the largest population in this study. Thirteen of the whole included children underwent second operation (VPS) for continuous ventricular enlargement after etV. Compared with the successful etV, the mean age of the patients with failure etV is $0.49 \pm 0.41$ years, and there is statistically significant $(\mathrm{P}<0.05)$. One presented subdural hematoma 1 month after et $\mathrm{V}$ because of over-drainage. The mean cost of etV for hydrocephalus is $24139.86 \pm 21085.65$ yuan. Conclusion: Compared with VPS, ETV is an ideal optional for treating pediatric patients with non-communicating hydrocephalus. Despite relatively high hospital expenditure, less postoperation complications and non-foreign entity implanted brought from etV overweigh that of VPS. Younger infants are more likely to undergo the second operation.

\section{2}

Long-term ICP profile after endoscopic third ventriculostomy

Sebastian Antes, Christoph A. Tschan, Joachim Oertel

Department of Neurosurgery, Saarland University Medical Center and Saarland University Faculty of Medicine, Homburg / Saar, Germany

Introduction: Endoscopic third ventriculostomy (ETV) has become the treatment of choice for occlusive hydrocephalus. As reduction or normalization of ventricular size often requires a certain time after surgery, there is actually no objective parameter indicating etV success of failure. Therefore, the aim of the study was to perform long-term intracranial pressure (ICP) monitoring after endoscopic treatment to identify parameters predicting the later clinical result of the procedure.
Methods: Seventeen telemetric ICP measurement devices (Raumedic Neurovent P-tel) were inserted in the frontal brain parenchyma of patients suffering from occlusive hydrocephalus. These implantations were always performed parallel to etV. After endoscopy patients were regularly followed-up with telemetric ICP measurements. Mean ICP values by day and night, maximum and minimum ICP peaks, the pulse-pressure amplitude as well as the distribution of the values were registered and analyzed. Results: In this cohort, ETV succeeded in 11 and failed in 6 patients. Reduction of the mean ICP could not be proven being a correlative parameter for clinical improvement. Successful surgery was rather associated to reductions of the maximum ICP peaks and the pulse-pressure amplitude. In contrast, no changes or even increases in mean ICP, maximum and minimum values and the pulse-pressure amplitude were indicative for failed endoscopic procedures.

Conclusions: Telemetric long-term monitoring could reveal differences in ICP profile between ETV-responders and -nonresponders.

\section{3}

Endoscopic lavage for posthemorrhagic hydrocephalus in neonates; Effects of treatment and comparison to historical controls

Ulrich-Wilhelm Thomale ${ }^{1}$, Christoph Bührer ${ }^{2}$, Hannes Haberl ${ }^{1}$, Matthias Schulz ${ }^{1}$

${ }^{1}$ Pediatric Neurosurgery, ${ }^{2}$ Department of Neonatology, Charité Universitätsmedizin Berlin, Berlin, Germany

Objective: Intraventricular hemorrhage (IVH) in neonates causes neurological morbidity due to direct brain tissue damage and a consecutive hydrocephalus. We investigated the effect of endoscopic lavage (EL) on posthemorrhagic hydrocephalus in neonates compared to a historical cohort.

Methods: Between 9/2010 and 12/2012 19neonates with IVH were treated with EL in order to clear the ventricles from blood clots and protein overload. Indication for surgery was IVH with active hydrocephalus. Warmed Ringers solution was used under endoscopic vision to clear the ventricles. This cohort was compared to 11 neonates with IVH and hydrocephalus who were treated conventionally before changing our protocol to EL (2/2008-7/2010). Possible complications, the need for further CSF diversion and for subsequent shunt revisions were evaluated. Results: Both groups were similar in age of birth, body weight, grade of hemorrhage and age as well as body weight at possible shunt surgery. For both groups at time of surgery the cranial ventricular indices were elevated above P97 in all patients. 4 patients received a second EL via a contralateral burr-hole. No significant perioperative complications after EL occurred. In the follow-up period of both groups significant differences between both groups were seen in shunt rate (100\% vs. EL:58\%), need of revisions (33 vs. EL:24) and formation of multiloculated hydrocephalus (4 vs. EL:0).

Conclusions: EL is feasible in neonates with posthemorrhagic hydrocephalus. Compared to historical controls we observed a benefit in shunt rate as well as possible complications after shunting. Further data is needed to evaluate long-term neurological outcome after EL.

\section{4}

New anatomical simulator for pediatric neuroendoscopic training Giselle Coelho ${ }^{1}$, Samuel Zymberg ${ }^{2}$, Marcos Lyra ${ }^{3}$, Benjamin Warf ${ }^{4}$ ${ }^{1}$ Pediatric Neurosurgery Center / CENEPE, Beneficência Portuguesa Hospital, São Paulo, Brazil; ${ }^{2}$ Department of Neurology and Neurosurgery, Paulista School of Medicine, Federal University of São Paulo, São Paulo, Brazil; ${ }^{3}$ Department of Gynecology, Federal University of Pernambuco, Pernambuco, Brazil; ${ }^{4}$ Department of Pediatric Neurosurgery, Boston Children Hospital, Harvard Medical School, Boston, USA

Introduction: The practice of neuroendoscopic procedures requires many years of training to obtain the adequate skills and abilities to perform this 
surgery, safely. The main goal of this study is to present a new pediatric neuroendoscopic simulator for training.

Methods: This real simulator was built with a synthetic thermo-retractile and thermo-sensible rubber called Neoderma ${ }^{\circledR}$ which, when combined with different polymers, produces more than 30 different formulas. These formulas present textures, consistencies and mechanical resistance similar to many human tissues. Silicon and fiberglass moulds, in the shape of the cerebral ventricles, constitute the basic structure of the neuroendoscopic module trainer.

Results: There were many possibilities to training: the endoscopic navigation to visualize and identify the anatomical structures (Septal and Thalamus Striatum veins, Monro foramen, Temporal horns and IV ventricle), to perform the choroid plexus coagulation and the third ventriculostomy. The presence of intraventricular lesions, simulating tumors, allowed their resection with bleeding.

Conclusion: It's important to emphasize that is possible to perform with this simulator not only the rigid but also the flexible endoscopy, with good correspondence to reality and no risks. Notable future perspectives can be considered regarding this new pediatric simulator, for example: to improve the learning curve for non experienced neurosurgeons and to spread the flexible endoscopy technique whose knowledge of its application is still restricted to few developed countries.

\section{5}

\section{Clinical features of occlusion of etV}

Kenichi Nishiyama ${ }^{1}$, Atsuko Harada ${ }^{2}$, Masakazu Sano ${ }^{1}$, Junichi Yoshimura $^{1}$, Yukihiko Fujii ${ }^{1}$

${ }^{1}$ Department of Neurosurgery, Brain Research Institute, University of Niigata, Niigata, Japan; ${ }^{2}$ Department of Pediatric Neurosurgery, Takatsuki General Hospital, Takatsuki city, Osaka, Japan

Purpose: To clarify the clinical features of occlusion of etV.

Methods: Eleven patients received re-ETV in our institute between 1997 and 2012. We usually arranged a follow-up MRI within the first 3 months and every 6 months following initial etV. Changes in clinical symptoms and MR findings with time elapsed and endoscopic findings at the time of re-ETV were analyzed retrospectively.

Results: In-between periods of initial etV and re-ETV were from 10 to 792 days (median, 165). Following initial etV, five patients presented with consciousness disturbance developed to symptomatic within a month, however, the others with alerted level developed over 3 months. A lack of flow void at the floor of the third ventricle on sagittal T2WI was verified in all 8 patients obtained on MRI before the onset of any symptoms. They became aware of the symptom from 90 to 570 days (median, 120) following MR studies. Endoscopic findings demonstrated membranous obstruction of the third ventricular floor in 8 and newly formed arachnoid trabeculae below the floor in 3. Symptoms appeared 304 days after etV in the former and 42 days in the latter on average. Conclusions: Occlusion of etV is sorted from early to late phase. In some cases, a few months may be taken to change into symptomatic after occlusion of etV. The forms of occlusion are classified into membranous obstruction of the ventricular floor and newly formed arachnoid trabeculae. Symptoms appear early in the latter.

\section{6}

\section{Effectiveness of endoscopic third ventriculocisternostomy} in hydrocephalic children under 2 years of age

Ruben Dammers, Lana Fani, T. H. Rob de Jong, Marie Lise C. van Veelen Department of Pediatric Neurosurgery, Erasmus MC, Sophia Children's Hospital, Rotterdam, The Netherlands

Background: Treating hydrocephalus can be difficult in children under the age of 2 years. In this retrospective cohort study, we analyzed children under the age of 2 years with hydrocephalus undergoing an endoscopic third ventriculocisternostomy (ETV) with respect to etV outcome.
Methods: In 59 consecutive patients under the age of 2 years, an etV was performed between 1999 and 2010. Demographics, etiology of hydrocephalus, and radiological data were extracted retrospectively from the patients' medical records and operative reports and related to outcome. ETV Success Score (ETVSS) as proposed by Kulkarni et al. (J Pediatr 2009;155(2):254-9) was used to retrospectively calculate the probability of success related to the actual outcome.

Results: In this series, $42.4 \%$ of patients had a successful etV. The failed etV patients appeared to be younger $(0.52$ vs. 0.86 year, $\mathrm{p}=0.005)$, and when using a cutoff age of 6 months only, five out of 32 infants had a successful etV $(\mathrm{p}=0.002)$. Of the five patients with a high probability of etV success, four $(80 \%)$ were indeed successfully treated with etV $(\mathrm{p}=0.049)$. Conclusions: Our data confirm the overall ineffectiveness of an etV in children under the age of 6 months. Nevertheless, using the etVSS is recommended to aid in the decision-making process even in patients under the age of 6 months.

\section{7}

Combined intraoperative-MRI and navigated endoscopy in children with multiloculated hydrocephalus and complex cysts: a series-based feasibility study

Paraskevopoulos Dimitrios, Biyani Naresh, Constantini Shlomo, Beni-Adani Liana

Dana Children's Hospital (TASMC) and Tel-Aviv University, Tel-Aviv, Israel

Introduction: Neuroendoscopy is an important tool in the treatment of Hydrocephalus, however, in cases of distorted anatomy and multiloculated cysts, endoscopy alone might be problematic as for appropriate orientation and re-evaluation of brain shift. The aim of this project was to assess the feasibility and efficacy of intraoperative MRI (ioMRI) guided, navigated neuroendoscopy in infants.

Patients and methods: Five infants (ages 6-14 months) operated for multicystic hydrocephalus presenting with shunt malfunction (4 patients) and a Quadrigeminal Fetal arachnoid cyst (1 patient), were followed 2-9 years. For all infants ioMR (0.12-Tesla PoleStar N-10 / 0.15-Tesla PoleStar N-20) was combined with navigated endoscopy. E-steady, T1 and T2 sequences were used ( $24 \mathrm{sec}-3.5 \mathrm{~min})$.

Results: The ioMRI system provided clear images that correlated with the endoscopic appearance of the cystic membranes in all patients, and was helpful in determining trajectories and redefining targets. The intraoperative MR images documented brain shift and change of CSF spaces during surgery. There were no intraoperative complications or technical difficulties of visualization. No infection or any other immediate postoperative complication occurred. The infant with the quadrigeminal cyst is still shunt free, and the patients with multicystic HCP have 1-2 shunts each.

Conclusions: IoMRI enabled transforming a blind conventional stereotactic procedure into a visually controlled navigated procedure by updating and redefining new targets during surgery, allowed accurate navigation of the endoscope, and minimized number of compartments in all patients. No complications related to the use of ioMRI occurred.

\section{8}

Microsurgical outlet restoration in isolated fourth ventricular hydrocephalus: a single-institutional experience

Lena Armbruster ${ }^{1}$, Mathias Kunz ${ }^{1}$, Birgit Ertl-Wagner ${ }^{2}$, Jörg-Christian Tonn ${ }^{1}$, Aurelia Peraud ${ }^{1}$

${ }^{1}$ Department of Neurosurgery, Ludwig-Maximilians-University Munich, Munich, Germany; ${ }^{2}$ Department of Radiology, Ludwig-MaximiliansUniversity Munich, Munich, Germany

Introduction: Isolated fourth ventricles as a consequence of shunted posthemorrhagic hydrocephalus can cause significant brainstem compression and subsequent clinical deficits in children. Several treatment 
options have been described. We report the clinical and radiological outcome after microsurgical fenestration of fourth ventricular outlet foramen via a suboccipital approach.

Methods: In 9 patients (age, 9 to 87 months; median age, 21 months), microsurgical reopening of formerly occluded outlet foramen of the fourth ventricle was performed under electrophysiological monitoring. Pre- and postoperative clinical outcomes as well as radiological results are reported.

Results: Mean follow-up for all children was 25 months. Three children older than 3 years were less significantly involved, the remaining 6 showed tremendous signs of long tracts signs and lower cranial nerve deficits. All children exhibited a remarkable improvement of their preexisting neurological deficits post surgery. Despite successful fenestration one child required additional internal drainage of fourth ventricle to the lateral ventricles due to malabsorption. Median diameters of the fourth ventricle changed markedly after surgery with a.p. extension

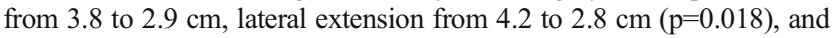
craniocaudal extension from 5.8 to $4.7 \mathrm{~cm}$, respectively. Also the pontine a.p. diameter increased significantly from 0.8 to $1.5 \mathrm{~cm}(\mathrm{p}=0.022)$.

Conclusions: The clinical and radiological outcomes after microsurgical fenestration in children with an isolated fourth ventricle are very promising. This treatment modality is a safe and effective shunt-free option when electrophysiological monitoring and thorough preoperative neuroradiological work-up is applied.

\section{9}

\section{Transaqueductal fenestration of the Lamina terminalis using} a flexible endoscope

Sonja Vulcu ${ }^{1}$, Hayk Bloutian ${ }^{1}$, Kurt Becker $^{2}$, Joachim Oertel

${ }^{1}$ Neurochirurgische Klinik, Universitätsklinikum des Saarlandes; ${ }^{2}$ Institut für Anatomie, Zellbiologie und Entwicklungsbiologie, Universität des Saarlandes

Introduction: Recently, the authors presented the transventricular, transforaminal fenestration of the Lamina terminalis (LT) as an alternative to standard endoscopic third ventriculostomy (ETV). Fenestration was feasible with a flexible and a rigid endoscope but with the hazard of fornical contusion due to the steep angle to LT. To avoid contusion and provide a flatter angle, the authors now investigate the feasibility of LT fenestration using a flexible endoscope via a transaqueductal approach. Methods: Ten cadaver specisms underwent LT fenestration with a small flexible endoscope (diameter $2.7 \mathrm{~mm}$ ) through a suboccipital approach. After passing the fourth ventricle and the aqueduct, LT was fenestrated with the aid of grasping forceps and fogarty balloon catheter.

Results: Passage of the aqueduct and fenestration of LT succeeded in all cadavers with the flexible endoscope. No damage of the aqueduct or anatomical structures in the third ventricle occurred. Identification of anatomical landmarks in the third ventricle and even in the lateral ventricles after passing foramen of Monro was good possible.

Conclusion: The transaqueductal fenestration of LT with the flexible endoscope might present an alternative in cases where standard etV is not feasible. However, handling of the instruments for fenestration is more difficult in a flexible system; additionally, the optical image quality is much worse, therefore, this procedure should be reserved for experienced hands. To gain more experience and to draw definite conclusions, clinical application in a patient series is necessary in future.

\section{0}

\section{Endoscopic treatment of posterior fossa cyst through the aqueduct} of sylvius

Pierre-Aurélien Beuriat, Alexandru Szathmari, G. Capone, Carmine Motttolese

Department of Pediatric Neurosurgery, Neurologic and Neurosurgical Hospital Pierre Wertheimer, Lyon, France
Posterior fossa cysts are a common pathology in pediatric neurosurgery. We report our experience in the surgical management of this condition. The surgical procedure consists in a third ventricle ventriculocysternostomy associated with cystostomy of the posterior fossa cyst through the aqueduct of sylvius with the help of neuronavigation and a plexectomy.

Results: We can report very good evolution with disparition of intracranial hypertension clinical signs and Parinaud syndrome, reduction of the size of the cyst and a good neurological outcome. We have treated 16 children between 2000 and 2012. Four of them required a ventriculo peritoneal shunt after the endoscopic procedure.

Conclusions: Surgical management of posterior fossa cyst of any type (Blake pouch cyst, Dandy Walker malformation, arachnoid cyst...) can be done by a full endoscopic procedure associated a third ventricle ventriculocysternostomy, a cystostomy through the aqueduct of sylvius and a plexectomy. This procedure is effective and can reduce the need of ventriculo-peritoneal shunt.

\section{1}

Choroid plexus coagulation: is it useful?

José Aloysio CostaVal, Leopoldo Mandic Furtado

Pediatric Neurosurgery, Vila da Serra Hospital, Nova Lima, Brazil

Introduction: The aim of this study was to analyse the different benefits of choroid plexus coagulation in various etiologies of hydrocephalus.

Methods: In this prospective study, choroid plexus coagulation was performed in patients between January of 2011 and December of 2012. The inclusion criterion was the presence of large ventricular dilatation with septum pellucidum agenesis on brain MRI: considered a predictor of choroid plexus bilateral visualization during neuroendoscopy - a must in order to coagulate the entire choroid plexus of the lateral ventricles. In this study analyzed the following variables: patient distribution by age, gender, hydrocephalus etiology, procedure duration, length of hospital stay and mortality rate. As far as benefits are concerned, we considered the following: time without valve, hydrocephalus definitive treatment and head shape after the valve.

Results: Choroid plexus coagulation was performed in nine patients, three girls and six boys (67\%). Among the hydrocephalus etiologies there were: Dandy-Walker syndrome in 1 case $(0.11 \%)$; type II Chiari in 2 cases $(0.22 \%)$, Hydroanencephaly $(0.11 \%)$, semilobar holoprosencephaly $(0.33 \%)$, and aqueduct stenosis with extreme hydrocephalus $(0.22 \%)$. We had to implant the valve in all the cases of this series, and the mean residence time without the valve was three months.

Conclusions: The choroid plexus coagulation was effective for the temporary control of hydrocephalus, time gain until valve implant and better cranial cosmetic results after the valve implant.

102

Placement of peritoneal catheter of VP shunt under laparoscopic guidance

Ryuji Ishizaki, Masashi Kitagawa, Takafumi Wataya, Yuzuru Tashiro Department of Neurosurgery, Shizuoka Children's Hospital, Shizuoka, Japan

Introduction: Laparoscope has recently given us an optical assistance in the operative procedure of inserting peritoneal catheter of VP shunt. The guidance can reduce a risk of intestinal injury, supply a better placement of the catheter, and give a chance to confirm the patency of whole system of new VP shunt. The setting of laparoscope was, at first, supported by general surgeons, but lately succeeded by ourselves.

Materials and Methods: We have retrospectively reviewed 44 cases with VP shunt under laparoscopic guidance about the operative time, complication and shunt outcome in our hospital from 2009 to 2012. Then, we have compared the data between two groups in the procedure of laparoscopic assistance; 22 cases with technical supports by general surgeons, and 22 cases by ourselves without the supports. 
Results: Under the guidance, we could successfully choice a piercing point of needle introducer and an advancing direction of the catheter to avoid adhesive portions of peritoneum and intestine suffered from several times of shunt revision. We had no operative complications and no shunt revisions in the follow-up period of all patients. There were no significant differences between the groups regarding operative time and shunt outcome. Conclusions: The results reveal that the placement of peritoneal catheter of VP shunt with laparoscopic assistance is useful and safe with minimal invasion. If neurosurgeons could be well-trained the procedure of laparoscopic setting, there might be no differences between with the support of general surgeon and without the support.

\section{3}

Fixed-pressure gravity-assisted valves and adjustable differential pressure valves in the treatment of pediatric hydrocephalus - a single center study of valve performance in the clinical setting

Thomas Beez, Sevgi Sarikaya-Seiwert, Lina Bellstädt, Hans-Jakob Steiger

Department of Neurosurgery, Heinrich-Heine-University, Düsseldorf, Germany

Introduction: Previous studies have established risk factors for shunt failure in children. The role of valve type as a determinant of complications and outcome remains unclear. Aim of this study was to compare the fixed-pressure paediGAV and the programmable Codman Hakim valve in the clinical setting.

Methods: We conducted a retrospective review of patients $<16$ years who underwent primary implantation of a ventriculoperitoneal shunt with either valve type between January 2005 and December 2010. Shunt survival analyses were performed to identify variables associated with risk of failure.

Results: Of the 44 patients in the paediGAV cohort, $50 \%$ reached the endpoint of shunt failure with a mean time to failure of 7 months. The Codman Hakim cohort comprised 29 patients, of which 55\% experienced shunt failure with a mean time to failure of 8 months. Stratified analysis identified young age at implantation and hemorrhage as etiology of hydrocephalus as risk factors for failure. Shunt survival analysis revealed no significant difference with regard to valve type.

Conclusions: This study confirmed important risk factors for shunt failure in children. Despite certain limitations, similar findings for both valves were obtained. Valve type does not seem to influence risk of shunt failure. Prospective, randomized and controlled trials are required to validate these results.

\section{4}

\section{Real-time continuous ultrasound-guided placement of ventricular catheters in children}

Thomas Beez, Sevgi Sarikaya-Seiwert, Hans-Jakob Steiger, Daniel Hänggi

Department of Neurosurgery, Heinrich-Heine-University, Düsseldorf, Germany

Introduction: Placement of ventricular catheters is a common procedure. The freehand technique is mainly used, which relies on anatomical landmarks and general coordinates for burr hole and catheter trajectory. The rate of inaccurate placement reaches $25 \%$, often leading to dysfunction. We thus evaluated real-time continuous ultrasound guidance, which allows for intraoperative visualization of anatomy, catheter trajectory and final position.

Methods: An Aloka burr hole probe (3.0-7.5mHz) was used. Ventricular size, midline shift and catheter position were assessed on CT or MRI. Ventricular size was quantified by frontal occipital horn ratio (FOHR). Ventricular catheter position was graded as I (= optimal), II (= catheter tip lying in contralateral ventricle or touching ventricular structures) or III (= misplaced).
Results: 21 patients were enrolled, with a mean age of 6.2 years (range 0 18 years). $18 \mathrm{CSF}$ shunts and $3 \mathrm{EVD}$ were inserted via either a frontal $(\mathrm{n}=$ $14)$ or occipital $(n=7)$ approach. Underlying pathology was posthemorrhagic hydrocephalus $(\mathrm{n}=9)$, occlusive hydrocephalus $(\mathrm{n}=3)$, acute hemorrhage or trauma $(n=3)$, malformation $(n=4)$ and other $(n=2)$. Mean preoperative FOHR was 0.45 (range: $0.28-0.71$ ). Grade I position was achieved in 7, grade II in 13 and grade III in 1 patient.

Conclusions: Real-time continuous ultrasound-guided placement of ventricular catheters significantly reduced the rate of misplacement to only $5 \%$. Controlled trials are required to evaluate influence on outcome, e.g. shunt survival. However, ultrasound appears to dramatically increase precision of ventricular catheter placement compared to freehand technique.

\section{5}

Advantages and disadvantages of temporary treatment with bedside-External Ventricular Drainage (EVD) in preterm babies with hydrocephalus secondary to IVH

Liana Beni Adani, Shlomi Constantini, Sigal Freedman, Inbal Golan Tripto

Pediatric Neurosurgery, Dana Children's Hospital, Tel-Aviv Sourasky Medical Center and the TA University (TAU), Israel

Background: Intraventricular hemorrhage (IVH) is a major complication of prematurity. A significant number of infants subsequently develop post-hemorrhagic hydrocephalus (PHH). Several methods have been proposed for the temporary/early treatment of $\mathrm{PHH}$, none of which were ideal either due to high complication rate or low efficiency. We established a protocol in which bedside External ventricular drainage (EVD) was placed as a temporary treatment for $\mathrm{PHH}$.

Objective: to study the efficiency and safety of bedside EVD in hydrocephalic low birth weight babies as a temporary treatment until they reach appropriate weight for definite shunt operation.

Methods: in a prospective study, 60 hydrocephalic preterm babies from 10 NICU departments were treated with bedside non-tunneled EVD by our senior neurosurgeons (overall $>220$ procedures). A $23 \mathrm{G}$ venflon was inserted after US to a lateral ventricle and connected to a sterile bag for up to 4 days to allow spontaneous drainage of CSF.

Results: Mean gestational age was $28.4 \mathrm{w}$ and mean birth weight was 1113.5 g. $92 \%$ had severe IVH (grade $3-4$ ). The first EVD was preformed at a mean age of 34 days and number of EVDs for a baby ranged from 15, median 2.6. The mean daily CSF volume removed by EVD was $60 \mathrm{ml}$, with a constant decrease in head circumference. $3.33 \%$ procedure-related infection rate, CSF over-drainage in $2 \%$, and CSF leak and dislodgment in $1.67 \%$ each, accounted for $6.67 \%$ minor complications that were treated easily, without any further implications. Over $90 \%$ of babies had VP eventually shunt operation.

Conclusion: bedside EVD is an efficient and safe therapy with relatively low and acceptable incidence of complications, as a temporary solution, until shunt operation is performed in most of the babies with progressive ventriculomegaly after IVH grade 3-4.

\section{6}

Paediatric Bactiseal external ventricular drains: a report on CSF sampling and time to infection

Digant Kamdar ${ }^{1}$, William Lo ${ }^{1}$, David Davies ${ }^{1}$, Marcelo Volpon ${ }^{1}$, Mitul Patel $^{1}$, Guirish A. Solanki ${ }^{1,2}$

${ }^{1}$ Department of Paediatric Neurosurgery, Birmingham Children's Hospital, Birmingham, UK; ${ }^{2}$ University of Birmingham, Birmingham, UK

Objectives: External ventricular drains (EVD) carry an infection risk, however no current guidelines regarding timing and frequency of CSF sampling exist. We aim to identify the length of time that EVDs left in situ remained infection free and the timing of CSF sampling. 
Patients: 70 (M-40, F-30) children underwent Bactiseal EVD insertion and CSF sampling between 2007 and 2011. Median age $4(12 \mathrm{~d}-15)$ years. Positive CSF culture at insertion and repeat/second EVD insertions were excluded. Primary outcome measure: Positive CSF culture. Shunt-related and non-shunt related EVD were compared.

Results: $68.6 \%$ were non-shunt related, including 40\% EVD insertions for neoplasm-related hydrocephalus. Shunt related EVD (31.4\%) included $17 \%$ shunt infections, $11 \%$ malfunctions. $6 \%(4 / 62)$ of children became CSF positive on days 5, 9, 28, 31. 75\% (3/4) grew Staphyloccocus Aureus. Infection in non- shunt related EVD was present in $2.2 \%(1 / 46)$ and in $19 \%(3 / 16)$ of shunt related EVD $(\mathrm{p}<0.02)$. Median time to infection was 9 days in the shunt related group and 28 days in the non-shunt related cases. Using the Kaplan-Meier survival curve, the daily probability of CSF remaining culture-negative was $>92 \%$ until the $9^{\text {th }}$ day post EVD insertion.

Conclusions: 1. Low infection frequency in non-shunt related EVD does not support routine CSF sampling in this subgroup. 2. Time-to-infection in non-shunt related EVD is longer than in shunt-related EVD. 3. Overall low CSF infection rate $(6.2 \%)$ does not support regular sampling of CSF before day 5. 4. Over $92 \%$ Bactiseal EVD survival to day 9 suggests delayed need for EVD replacement.

\section{Vascular disease, Trauma and infection}

107

\begin{abstract}
Arterial Spin labeling MRI: a new imaging tool for arteriovenous malformations management

Thomas.Blauwblomme ${ }^{1,2}$, Nathalie.Boddaert ${ }^{2,3}$, Francis.Brunelle ${ }^{2,3}$, Olivier.Naggara $^{2,3}$, Marie.Bourgeois ${ }^{1}$, Federico.DiRocco ${ }^{1,2}$, Laurent.Selek ${ }^{1}$, Stéphanie.Puget ${ }^{1,2}$, Christian Sainte-Rose ${ }^{1,2}$, Michel.Zerah ${ }^{1,2}$

${ }^{1}$ Assistance Publique Hôpitaux de Paris, Service de Neurochirurgie Pédiatrique, Hôpital Necker Enfants Malades, Paris, France; ${ }^{2}$ Université Paris Descartes, Sorbonne Paris Cité, Paris, France; ${ }^{3}$ Assistance Publique Hôpitaux de Paris, Service de Neuroradiologie, Hôpital Necker Enfants Malades, Paris, France
\end{abstract}

Introduction: Arterial Spin labeling is a recent perfusion sequence using labeled arterial protons as an endogenous perfusion agent to quantify cerebral blood flow (CBF). As cerebral AVM are high flow lesions, we postulated that ASL could be useful in AVM diagnosis and follow-up.

Methods: Studies were performed during the last 2 years on a 1,5T General Electric MRI. Following sequences were acquired: 3DT1, 3DT2, 3DTOF, and gadolinium injection. We used pulsed continuous ASL in axial scan plane with port label delay of 1025 seconds, with 40 slices of $4 \mathrm{~mm}$. All patients had a conventional angiography. 13 patients with a cerebral AVM and PC-ASL imaging were included.

Results: 7 patients were recently diagnosed with a brain AVM, 4 patients had been previously treated by surgery or endovascular treatment, 2 patients were followed after radiosurgery. ASL showed hyperperfusion inside the AVM nidus in $100 \%$ of the cases. Mean nidal $\mathrm{CBF}$ was $143 \mathrm{~mL} /\left(100 \mathrm{~min} \mathrm{~cm}^{3}\right)$, and nidus size ranged between 0,4 and $12,2 \mathrm{~cm}^{2}$. Image fusion between 3DT1Gd and PC-ASL sequences showed precise reproducible co-localization of the nidus. After radiosurgery, CBF decreased in both patients from 200 to 94 and from 151 to $93 \mathrm{~mL} /\left(100 \mathrm{~min} \mathrm{~cm}^{3}\right)$ after 17 and 14 months respectively.

Conclusion: ASL is a promising tool to diagnose AVM after intra cerebral hemorrhage and to detect recurrence after treatment. Measurement of $\mathrm{CBF}$ and nidus size will quantify objectively the efficacy of radiosurgery and may replace gadolinium injection.

\section{8}

Pediatric arteriovenous malformations: epidemiology, treatment and outcome. A retrospective series of 97 consecutive cases

Thomas Blauwblomme ${ }^{1,2}$, Francis Brunelle ${ }^{2,3}$, Olivier Nagarra ${ }^{2,3,4}$, Marie Bourgeois ${ }^{1}$, Federico DiRocco ${ }^{1,2}$, Laurent Selek ${ }^{1}$, Stéphanie Puget $^{1,2}$, Philippe Meyer ${ }^{5}$, Christian Sainte-Rose ${ }^{1,2}$, Michel.Zerah ${ }^{1,2}$

${ }^{1}$ Assistance Publique Hôpitaux de Paris, Service de Neurochirurgie Pédiatrique, Hôpital Necker Enfants Malades, Paris, France; ${ }^{2}$ Université Paris Descartes, Sorbonne Paris Cité, Paris, France; ${ }^{3}$ Assistance Publique Hôpitaux de Paris, Service de Neuroradiologie, Hôpital Necker Enfants Malades, Paris, France; ${ }^{4}$ Service de Neuroradiologie, Hôpital SainteAnne, Paris, France; ${ }^{5}$ Assistance Publique Hôpitaux de Paris, Service d'Anesthésie-Réanimation Pédiatrique, Hôpital Necker Enfants Malades, Paris, France

Introduction: The aim of this study was to analyze pediatric cerebral AVM treatment and outcome.

Methods: A single center retrospective study was done. Clinical presentation, imaging and outcome were analyzed. Statistical analysis was performed with Chi-square test, Mann-Whitney test and Kaplan Meier model.

Results: 97 patients (mean age 9,5 years) were treated between 1995 and 2012. Initial presentation was intra-cerebral hemorrhage (ICH) in $80 \%$ of the cases requiring surgical evacuation of the hematoma in $51 \%$ of the cases. Surgical indication significantly correlated with Glasgow coma scale $(<8)$, and hematoma volume $\left(>20 \mathrm{~cm}^{3}\right)$. The mortality rate of ruptured AVM was 6,5\%. 45 Patients were treated for a Spetzler-Martin grade I-II AVM. Overall success rate was $80 \%$. Surgery had the highest chance to treat the AVM in a single procedure $(p<0,001)$, with comparable neurological outcome. Despite multimodal treatment with embolization, gammaknife and/or surgery, cure rate of high-grade AVM was only $22 \%$. Rebleeding was noted in 9 cases with a mean interval of 21 months after first incomplete therapy. It was correlated with deep venous drainage and high-grade AVM ( $p=0,008$ and 0,007). Death occurred in 2/9 cases, and functional outcome was significantly worse after rebleeding $(p=0,046)$. One patient had true AVM relapse 9 years after surgery.

Conclusions: Our series supports surgical treatment for low-grade AVM. High-grade AVMs are challenging lesions, with a high rate of untreated patients, at risk of secondary bleeding and neurological impairment, even in adulthood.

\section{9}

Pediatric intracranial aneurysms: Characteristics, presentation, management and outcome

Yasser Orz ${ }^{1}$, Mahmoud AlYamany ${ }^{1}$, Zoheir Hassan ${ }^{1}$, Sultan AlQahtany ${ }^{2}$ ${ }^{1}$ Neurosurgery Department, ${ }^{2}$ Neurointervention Department, National Neuroscience Institute, King Fahad Medical City, Riyadh, Saudi Arabia

Background: Pediatric aneurysms are rare and poorly understood as compared to those in adults. The aim of our study is to characterize the clinical, imaging, presentation, management, and outcome of patients younger than 18 years diagnosed with intracranial aneurysms at our institute.

Materials and methods: A retrospective review of patients presented with intracranial aneurysms managed in our center from 2007-2013.

Results: Of 170 patients with intracranial aneurysms managed in our institute during that period, 14 pediatric patients (8.2\%) harboring 24 aneurysms were treated using microsurgical or endovascular techniques. Nine patients $(65 \%)$ presented with subarachnoid hemorrhage. Most of the aneurysms located at the anterior circulation (87.5\%). Eight patients $(58 \%)$ had their aneurysms located at the carotid artery bifurcation (CAB). Most of the aneurysms were small in size with wide necks and irregular shape, 3 patients had giant non ruptured aneurysms. One patient had 8 aneurysms located at different locations and he experienced 2 
attacks of SAH from 2 different aneurysms at 4 years interval and he died due to the second attack, another patient had $2 \mathrm{SAH}$ attacks with 7 years interval the second attack was from ruptured do novo right PCom aneurysm. Most of the patients $(86 \%)$ treated by microsurgical clipping of their aneurysms. Twelve patients $(86 \%)$ had good outcome and only one patient died in our series.

Conclusions: Pediatric Intracranial aneurysms pose considerable diagnostic and therapeutic challenges. Good outcome was achieved with both surgical and neurointerventional management of pediatric patients with intracranial aneurysms. Long life follow up with either CTA or angiogram should be considered for these patients.

\section{0}

\section{Epidemiology of moyamoya disease in Taiwan: a nationwide population-based study}

Meng-Fai Kuo ${ }^{1}$, Pei-Chun Chen ${ }^{2}$, Shih-Hung Yang ${ }^{1}$, Kuo-Liong Chien ${ }^{2}$ ${ }^{1}$ Department of Neurosurgery, National Taiwan University Hospital, College of Medicine, National Taiwan University, ${ }^{2}$ Institute of Epidemiology and Preventive Medicine, College of Public Health, National Taiwan University

Introduction: Population based studies on incidence and patient characteristics of moyamoya disease (MMD) in Taiwan are undertaken.

Methods: Using the inpatient claims of the Taiwan National Health Insurance program, we identified persons who had an initial hospitalization with a discharge diagnosis of MMD during 2000 to 2010. Patients with MMD who had risk factors of moyamoya syndrome were excluded. The temporal trends in incidence and the clinical and epidemiological features of MMD were described by age.

Results: During the 11-year period, the incidence of MMD (per 100,000 person-years) was 0.28 among children aged $\leq 6$ years, 0.41 in those aged $7-12$ years, 0.22 in those aged $13-18$ years, and 0.18 in those $>18$ years. The incidence increased from 0.23 in 2000 to 0.58 in 2010 in $\leq 6$-year old group. The corresponding figures for subjects aged 7-12 years were 0.31 and 0.44 ; for those aged 13-18 years, 0.14 and 0.47 , and for those $>18$ years, 0.13 and 0.25 . Compared to the adults, the pediatric patients tended to present with epilepsy ( $14.4 \%$ vs. $4.7 \%, p \leq 0.001)$ instead of cerebral infarction and hemorrhagic stroke. Adults subjects tended to have history of hypertension and diabetes. A total of 2 cases $(4.1 \%)$ of pediatric patients had been diagnosed with Thalassemia, but no adult had the disease.

Conclusion: Incidence of moyamoya disease increased during the past decade in Taiwan, a Southeast Asian country, though the incidence is much lower than that of Japan. The mode of presentation was different between pediatric and adult population.

\section{1}

Retinoic acid signaling in endothelial progenitor cells of moyamoya disease: decreased Raldh2 expression and rescue from defective angiogenesis by retinoic acid

Ji Yeoun Lee ${ }^{1}$, Youn Joo Moon ${ }^{1}$, Woong-Yang Park ${ }^{2,3}$, Kyu-Chang Wang ${ }^{1}$, Jae Kyun Jung ${ }^{3}$, Hyun Seung Kang ${ }^{1}$, Ji Hoon Phi ${ }^{1}$, Seung-Ki $\mathrm{Kim}^{1}$

${ }^{1}$ Division of Pediatric Neurosurgery, Department of Neurosurgery, Children's Hospital, Seoul National University, College of Medicine, Seoul, Korea; ${ }^{2}$ Department of Molecular Cell Biology, Sungkyunkwan University, School of Medicine, Suwon, Korea; ${ }^{3}$ Translational Genomics Laboratory, Samsung Genome Institute, Samsung Medical Center, Seoul, Korea

Moyamoya disease (MMD) is a common etiology for childhood stroke. Due to the lack of an animal model, elucidation on the pathogenesis had been limited. Recently, endothelial progenitor cell (EPC) has been shown to play a key role, enabling in vitro research on MMD. Gene expression profiling and in vitro functional experiments were performed using EPC to explore the pathogenesis of MMD. EPC from MMD patients and normal controls were cultured. Transcriptional profiling was performed in MMD EPC $(n=5)$ and normal EPC $(n=5)$. A total of 396 differentially expressed genes were identified, among which retinaldehyde dehydrogenase 2 (Raldh2) was noted to be significantly down-regulated in MMD EPC. Since Raldh2 is the enzyme involved in retinoic acid (RA) synthesis, further evaluation was done by performing tube formation assay after treatment of RA in MMD EPC $(n=3)$. Defective tube formation capability of MMD EPCs was restored after RA treatment. When Raldh2 was knocked down in normal EPC $(n=3)$ using siRNA, in vitro capillary formation was decreased. This functional impairment was recovered when RA was treated to normal EPC transfected with Raldh2 siRNA. Decreased in vitro tube formation by down regulation of Raldh2 and sequential recovery of the tube formation ability by RA treatment was validated using human umbilical vein endothelial cells. This study shows RA deficiency caused by under-expression of Raldh2 in EPC of MMD patients plays a key role in functional impairment of MMD EPC, contributing to the pathogenesis of MMD. It brings forth a potential role of RA as a therapeutic agent for MMD.

\section{2}

Vascular lesions observed in pediatric patients with craniopharyngioma

Reizo Shirane ${ }^{1}$, Tomomi Kimiwada ${ }^{1}$, Ayumi Narisawa ${ }^{1}$, Toshiaki Hayashi $^{2}$, Teiji Tominaga ${ }^{3}$

Departments of Neurosurgery, ${ }^{1}$ Miyagi children's hospital, ${ }^{2}$ Sendai City hospital, ${ }^{3}$ Tohokuuniversity, Sendai, Miyagi, Japan

Vasculopathy is not rare complication in pediatric patients with craniopharyngioma. Surgical manipulation, radiation therapy, or possible chemical reaction of cranippharyngiomas may cause vascular lesions. Between 1993 and 2011, 44 patients under the age of 20 underwent radical resection of craniopharyngiomas in our hospital. Seven cases received radiation therapy and 15 cases received gamma knife surgery after tumor resection. All cases received routine examination by MRI and MRA. At a mean follow-up time of 9.8 years, four cases of vasculopathy were experienced. Fatal SAH from the anterior cerebral artery dissecting aneurysm was observed in 12-year-old girl, four years after radical resection followed by gamma knife surgery of recurrent tumor. Moyamoya syndrome was observed in two cases. First case was not related to radiation therapy. Fifteen-year-old girl complained severe headache 11 years after radical resection of suprasellar tumor. MR revealed small cystic recurrent tumor at the frontal base and Moyamoya syndrome. STA-MCA bypass surgery followed by tumor removal resolved her symptoms. Second case was 13-year-old girl with the history of two radical resections and radiation therapy. Moyamoya syndrome developed four years after the radiat ion therapy. Leg motor weakness and headaches were appeared and STA-MCA bypass was performed. Cerebral infarction due to occlusion of right middle cerebral artery was observed in 3-yearold girl with huge cystic tumor in the right cerebral hemisphere surrounded by tough calcification. Her initial symptoms were seizure attacks and consciousness disturbance. Left hemiparesis was noticed after emergency cyst drainage.

Conclusions: Pediatric patients with craniopharyngioma should be reminded about the risk of development of vascular lesions.

\section{3}

Metabolic and inflammatory changes in the injured brain

Anthony Figaji ${ }^{1,2}$, Sinead Ross ${ }^{2}$, Ursula Rohlwink ${ }^{1,2}$, Graham Fieggen $^{1,2}$, Llewellyn Padayachy ${ }^{1,2}$, Jacob Hoffman ${ }^{2}$

${ }^{1}$ Division of Neurosurgery, ${ }^{2}$ University of Cape Town, Cape Town, South Africa

Introduction: Secondary mechanisms in pediatric traumatic brain injury (TBI) contribute substantially to death and disability but are little understood. Microdialysis can be used to study chemical substances in the brain. 
Recent developments have enabled examination of macromolecules, but this has not yet been applied in children. In this study we examined changes in energy metabolites and inflammatory mediators in children with TBI.

Method: Five children with severe TBI underwent monitoring with cerebral microdialysis. Metabolites examined by continuous bedside analysis included pyruvate, lactate, glucose, glycerol and glutamate. We used the Luminex multiplex bead array assay to conduct offline analysis of inflammatory mediators, including interleukins IL- $1 \alpha$, IL1- $\beta$, IL1-ra, IL-6, IL-8, and IL-10; VEGF and MCP-1. Results were correlated with brain and systemic physiology (such as intracranial pressure [ICP], brain oxygenation, serum glucose), and outcome.

Results: The lactate/puruvate $(\mathrm{L} / \mathrm{P})$ ratio was highest in patients who had a poor outcome, one because of ischemia, one likely because of mitochondrial dysfunction; and was a better discriminator of outcome than ICP. The L/P ratio was highest when brain oxygenation was abnormally low (likely ischemic) or abnormally high (likely dysregulated). Arterial and brain glucose were correlated $(\mathrm{r}=0.51, \mathrm{p}<0.0001)$. Brain glycerol was highest with contusions. Inflammation depended on timing after injury and whether cytokines were pro- or anti-inflammatory. There were many inter-individual differences but IL-6 and IL- 8 were most elevated.

Conclusion: Measurement of the chemical responses in the brain is possible in children and provides novel insights into secondary mechanisms of injury, with potential for targeted treatment.

114

Therapeutic hypothermia is not beneficial for pediatric patients with traumatic brain injury: A meta-analysis

Chenkai Ma, Xiaoguang He, Qifeng Li, Baocheng Wang, Jie Ma Department of Pediatric Neurosurgery, Shanghai Xinhua Hospital, Shanghai Jiao Tong University School of Medicine

Introduction: Hypothermia therapy shows its unique potential for reducing mortality in animal study and improving neurologic outcome in the patients with traumatic brain injury. However, therapeutic hypothermia for pediatric traumatic brain injury remains a controversial issue. To determine the effectiveness and safety of hypothermia treatment for pediatric traumatic brain injury patients, we conducted this meta-analysis. Methods: We analyses the data from MEDLINE, Pubmed, EMBASE and Cochrane Library by electronic searching. No limitation of language was selected for analysis. We extracted the mortality and adverse events from the published trials.

Results: Six clinical trials and 366 pediatric patients met our inclusion criteria. Pediatric patients with traumatic brain injury treated with hypothermia had more unfavorable outcome than those in the normothermia group (RR $1.73,95 \%$ CI 1.06 to 2.84), and this increased risk is statistically significant. Patients with therapeutic hypothermia are slightly likely to be induced by cardiac arrhythmia, though, the likelihood is also significant (RR 2.57, 95\% CI 1.01 to 6.54). Risk of pneumonia is no statistical difference between normothermia and hypothermia arms (RR $0.90,95 \%$ CI 0.73 to 1.12 ). Two of included trials have reported their detail randomization assignment.

Conclusions: Hypothermia may increase the risk of mortality in children with traumatic brain injury slightly and the ratio of cardiac arrhythmia after this hypothermia therapy is slightly higher than that in normothermia groups. In the future more randomized controlled trials and multicenter studies on the mechanism of therapeutic hypothermia are required.

\section{5}

\section{State of cerebrovascular autoregulation correlates to outcome} in severe traumatic brain injury

Martin U. Schuhmann ${ }^{1}$, Carmen Nagel $^{2}$, Ines Gerbig ${ }^{3}$, Ellen Heimberg ${ }^{3}$, Jennifer Diedler ${ }^{1}$

${ }^{1}$ Section of Pediatric Neurosurgery, Department of Neurosurgery, ${ }^{2}$ Department of Pediatric Surgery, ${ }^{3}$ Pediatric Intensive Care Medicine, Eberhard Karls University Hospital, Tübingen, Germany
Introduction: It could be shown in severe traumatic brain injury (TBI) in adults, that the functional status of the cerebrovascular autoregulation, determined via the cerbrovascular pressure reactivity index - PRx, clearly correlates to and predicts outcome. We investigated PRx in severe infant and pediatric TBI in correlation to outcome.

Methods: 10 patients (median age $2.8 \mathrm{y}$, range $1 \mathrm{~d}-13 \mathrm{y}$ ) with severe TBI $(\mathrm{GCS}<9)$ underwent longtime computerized ICP and $\mathrm{ABP}$ monitoring using ICM+ software to determine CPP and PRx continuously. Outcome was determined at 6 months using the Glasgow Outcome scale.

Results: Median monitoring time was $182 \mathrm{~h}$, range 22-356h. 6 patients needed decompressive craniectomy to control ICP during the course of treatment. Outcome: favorable outcome (GOS 4+5) $\mathrm{n}=4$, GOS $3 \mathrm{n}=3$, GOS $2 \mathrm{n}=1$, GOS $1 \mathrm{n}=2$. Autoregulation was lost in GOS $1+2$ patients. When dichotomized according to favorable (GOS4+5) versus unfavorable outcome (GOS 1-3) ICP and CPP were not different, however, PRx correlated well to outcome $(r=-0.79, p=0.006)$ and was significantly lower (means better autoregulation) in GOS4+5 than in GOS1-3.

Conclusion: Functionality and integrity of cerebrovascular autoregulation plays the same major role regarding outcome in infants and children as in adults and should be routinely determined. It carries an important prognostic value and, furthermore, seems to be the ideal parameter to guide treatment with the aim of improving cerebrovascular autoregulation.

116

Incidence of seizures on continuous EEG (cEEG) following traumatic brain injury in children

Michael H. Handler ${ }^{1,3}$, Kristy Wolter-Warmerdam ${ }^{3}$, Brent $\mathrm{O}^{\prime} \mathrm{Neill}^{1,3}$, Kevin Chapman ${ }^{2,3}$

${ }^{1}$ Department of Neurosurgery, University of Colorado School of Medicine, Aurora, CO USA $;{ }^{2}$ Departments of Neurology and Pediatrics, University of Colorado School of Medicine, Aurora, CO USA; ${ }^{3}$ Children's Hospital Colorado, Anschutz Medical Campus, Aurora, CO, USA

Introduction: Seizures may cause diagnostic confusion or add an additional metabolic burden after traumatic brain injury (TBI) in children. The incidence of EEG-confirmed seizures and subclinical seizures following TBI in children is not well known.

Methods: Data was collected over 3.5 years at a regional Pediatric Level 1 Trauma Center on all patients with TBI receiving cEEGs for 48 hours. $61 \%$ of patients with severe TBI received cEEG, as did certain patients with less severe injury and less rapid recovery than predicted. The hospital's cEEG database was merged with the trauma registry, and clinical details extracted.

Results: From 595 admissions of children with TBI, 142 patients underwent cEEG, 82 (57.7\%) with severe TBI (GCS 3-8), 14 (9.9\%) with moderate (GCS 9-12), and 43 (30.3\%) with mild (GCS 13-15). In 3, initial GCS was inadequately documented. Seizures were identified in $32.4 \%$ of patients; $23.2 \%$ had only subclinical seizures and $9.2 \%$ had clinical $+/-$ subclinical seizures. Independent predictors of subclinical seizures were abusive head trauma $(\mathrm{p}<0.001)$ and age $<2$ years $(p<0.001)$. Not predictive of subclinical seizures were seizures on scene, need for craniotomy, and initial GCS. Subclinical seizures correlated with increased hospital length of stay $(\mathrm{p}=0.007)$ but not length of ICU stay.

Conclusion: Continuous EEG identifies a significant number of subclinical seizures acutely after TBI. Victims of abusive head trauma and those under 2 years of age are particularly vulnerable. cEEG allows earlier appropriate intervention, and may mitigate secondary damage. 
117

\section{The rate of shunt-free hydrocephalus in tuberculous meningitis} in children

Tommy A. Nazwar, Wihasto Suryaningtyas, Muhammad Arifin Section on Pediatric, Department of Neurosurgery, Faculty of Medicine, Airlangga University, Soetomo General Hospital, Surabaya, Indonesia

Introduction: Hydrocephalus occurs in up to $85 \%$ of tuberculous meningitis (TBM) in children. Palur et al. made a grading classification for hydrocephalus due to TBM. But, there is little known about the percentage of children with TBM that was free from shunt after the extraventricular drainage (EVD). This series aims to study the probability of shunt-free after EVD in children with TBM.

Methods: Retrospective study using medical record of children with TBM was done in Soetomo General Hospital, Surabaya from 2011 to 2012. Data was collected to examine the incidence of intracranial TB with or without extra-cranial TB, the rate of primary shunting and EVD followed by shunting, Palur Grade of each patient, and the rate of shunt free patient after EVD.

Result: Medical records of 25 patients were reviewed. TBM was confirmed radiologically as it showed enhancement or multiple enhanced small nodules in the basal cisterns. Polymerase Chain Reaction (PCR)TB from CSF showed positive results in all patients. Twenty-one cases were pure intracranial TB and 4 other patients harbored TB in brain and lung. Fifty percent of cases was Grade 2, Grade 3 in $8,3 \%$ cases, and grade 4 in $41,7 \%$ cases. Primary VP shunts were implanted in 21 cases (84\%). Three cases (12\%) received EVD followed by shunt, and only one case $(4 \%)$ that was shunt free after EVD. There was no difference in severity between pure intracranial and Intra - extra cranial TB.

Conclusions: The rate of shunt dependent TBM hydrocephalus was high. Only few of them survive without shunt.

\section{8}

Suspected shunt infection in children: are inflammatory markers a good positive indicator?

Digant Kamdar $^{1}$, David J. Davies ${ }^{1}$, N. Wickramasinghe ${ }^{1}$, Mitul Patel ${ }^{1}$, Guirish A. Solanki ${ }^{1,2}$

${ }^{1}$ Department of Paediatric Neurosurgery, Birmingham Children's Hospital, Birmingham, UK, ${ }^{2}$ University of Birmingham, Birmingham, UK

Objectives: Shunt infection represents a significant paediatric morbidity. Inflammatory markers (CRP, WCC, platelet count and ESR) are used in decision-making in suspected shunt infections. We evaluate these markers in differentiating true shunt infections from other diagnoses at presentation.

Design: Retrospective review of admission and laboratory results (microbiology, haematology and biochemistry).

Subjects: 31 children, 20 boys and 11 girls, with suspected shunt infections were identified. Age range (1 week to 16 years).

Methods: Our paediatric referral database was searched for children presenting with suspected shunt infection between July 2006 and August 2011. Data from blood samples taken within 24 hours of admission, including haemoglobin, white cell count, CRP and platelet count was analysed. Microbiological samples from shunts were reviewed to confirm infection. Results: $15 / 31$ had proven shunt infections. The admission WCC ( $\mathrm{p}<$ $0.019)$, PLT $(p<0.011)$ and CRP $(p<0.005)$ were significantly higher in the proven infection group than the unproven group. A WCC greater than or equal to $10.45 \times 109 / 1$ resulted in an $80 \%$ sensitivity and $50 \%$ specificity, as did a CRP of $14 \mathrm{mg} / \mathrm{l}$. A platelet count of $420 \times 109 / 1$ resulted in an $80 \%$ sensitivity and $75 \%$ specificity.

Conclusion: CRP, WCC and PLT on admission were accurate predictors for microbiologically proven shunt infections, with threshold values of CRP 14, WCC10.45 and Plt 420. Inflammatory markers are invaluable in assessing suspected shunt infection at presentation. A larger, more sensitive review of our data is planned to improve the sensitivity and usefulness of these figures.
Epilepsy

119

Improving outcomes and avoiding complications in surgery for hypothalamic hamartoma

Harold L. Rekate, David J. Langer

The Chiari Institute; Cushing Neurosciences Institute; Hofstra Northshore LIJ School of Medicine Manhasset, NY; USA

Purpose: Removal or disconnection of hypothalamic hamartomas using a transventricular approach either using an open or an endoscopic approach has proven more effective in treating intractable epilepsy than has removal from a pterional approach. Rates of complication in attempting complete removal or complete disconnection from transventricular approaches have been high in very large hamartomas.

Design: This study is a retrospective review of patients with hypothalamic hamartoma and intractable epilepsy seen at a single center whose first approach at our center involved a modified orbito-zygomatic craniotomy using the optic tract as the superior limit of resection.

Results: There have been five patients with hypothalamic hamartomas greater than $2.5 \mathrm{~cm}$ whose connections to the hypothalamus have been both inside the third ventricle and inferior to the hypothalamus. There were four children ranging in age from 9 months to 4.5 years and one adult ( 27 years). They all presented with both gelastic and partial complex seizures and all four children also had precocious puberty. Complete removal was possible in all patients using pterional approach alone or part of a staged operative plan. No patient suffered permanent difficulties with sodum metabolism postop. All are seizure free with resolution of precocious puberty in the four children.

Conclusion: For very large hypothalamic hamartomas occurring within the third ventricle and in the interpeduncular cistern we recommend open pterional removal of the bottom of the mass to protect the pituitary stalk and hypothalamus as a first step.

120

Surgical treatment for pediatric hypothalamic hamartomas Baochen Wang, Chenkai Ma, Xiaoguang He, Qifeng Li, Jie Ma Department of Pediatric Neurosurgery, Xinhua Hospital, Shanghai Jiao Tong University, School of Medicine, Shanghai, China

Introduction: Hypothalamic hamartomas may be associated with gelastic seizures and a generalized epileptic encephalopathy, with cognitive and behavior decline. Resection was long regarded as too hazardous and unlikely to benefit seizure control.

Materials and methods: We reviewed our experience of surgical treatment for hypothalamic hamartomas with epileptic seizures. Lateral pterional, midline frontal through the transcallosal interforniceal approaches were compared. Mortality, morbidity, and efficacy of these neurosurgery procedures were considered. Our experience of pediatric neurosurgery is based on a retrospective analysis which enrolled 13 patients with $\mathrm{HH}$ and associated severe epilepsy between August 2007 and August 2011.

Results: Surgical treatments can lead to a dramatic reversal of the epileptic seizure. In our series, 8 kids have a follow-up superior to 3 years. Among those, 7 kids have an excellent result with partly or completely seizurefree. No permanent neurological complication has been observed so far. Young patients with severe epilepsy and cognitive/psychiatric co morbidity must be operated on as early as possible. Large $\mathrm{HH}$ above the floor of the third ventricle must be disconnected. Transcallosal interforniceal approach is proposed.

Conclusion: Our first results indicate that microsurgical resection is effective and safe. Longer follow-up is mandatory for a reliable evaluation of the role of resection. 
121

Brain tumors in children with refractory seizures - a long-term follow-up study after epilepsy surgery

Caroline Meyer ${ }^{1}$, Susanne Bartels ${ }^{1}$, Robert Sassen ${ }^{2}$, Christoph Schöne-Bake ${ }^{4}$, Marec von Lehe ${ }^{3}$

${ }^{1}$ Department of Neurosurgery, ${ }^{2}$ Department of Epileptology, University Hospital Bonn; ${ }^{3}$ Department of Neurosurgery, University Hospital Bochum; ${ }^{4}$ Department of Pediatrics, University Hospital Freiburg, Germany

Objective: To analyze the epileptological and neuro-oncological outcome after epilepsy-surgery in children with refractory seizures caused by brain tumors.

Methods: The data of 107 patients (mean age 12 years (1-17)) was analyzed. Neuro-oncological follow-up (FU) data was available from 70 patients $(65 \%)$. Sufficient seizure outcome data (FU $>11$ months) was available from 102 patients $(95 \%$, classified according to ILAEclassification).

Results: Mean FU of all patients was 119 months (1-284 months). Tumor localization was mostly temporal $(60 \%)$. Histologically, $57 \%$ were gangliogliomas, $24 \%$ gliomas, $14 \%$ dysembryoblastic neuroepithelial tumors, $5 \%$ Oligodendrogliomas; $77 \%$ were classified WHO ${ }^{\circ} \mathrm{I}, 19 \%$ $\mathrm{WHO}^{\circ} \mathrm{II}, 4 \% \mathrm{WHO}^{\circ} \mathrm{III}$. The rate of permanent unexpected neurological deficits was $5.6 \%$, no perioperative mortality. Moreover, there was a considerable rate of calculated visual field defects after temporal resections. Regarding neuro-oncological FU, 4 patients were treated with adjuvant radio-chemotherapy, two with an astrocytoma $\mathrm{WHO}^{\circ} \mathrm{III}$ and two with a recurrent ganglioglioma $\left(\mathrm{WHO}^{\circ} \mathrm{II}\right)$. One patient died from tumor growth (tumor-related mortality 1.5\%). At last available outcome, $82 \%$ of the patients were seizure-free (ILAE 1 ). $62 \%$ of these were without antiepileptic drugs (AED). Long-term seizure outcome was stable ( $81 \%$ after one year, $79 \%$ after 3 years, $85 \%$ after 10 years).

Conclusion: Surgical treatment of long-term epilepsy associated tumors in children is safe although calculated postoperative deficits occur especially after temporal-localized tumors. Seizure outcome is promising and the majority of seizure-free patients are without AED. The vast majority are benign tumors. However, malignant tumors and recurrent tumor growth occur and therefore tumor patients require neuro-oncological follow-up.

\section{2}

\section{Surgical outcome of epilepsy surgery on patients with tuberous sclerosis}

Dong-Seok Kim ${ }^{1}$, Kyu-Won Shim ${ }^{1}$, Eun Kyung Park ${ }^{1}$, Kook Hee Yang ${ }^{2}$, Seung-Woo Park ${ }^{3}$

${ }^{1}$ Department of Pediatric Neurosurgery, Severance Children's Hospital, Seoul, Korea; ${ }^{2}$ Department of Neurosurgery, National Health Insurance Service Ilsan Hospital, Koyang, Kyungkido, Korea; ${ }^{3}$ Department of Neurosurgery, Kangwon National University Hospital, Chunchun, Kangwondo, Korea

Objective: Tuberous sclerosis complex (TSC) is a multisystem genetic disorder with variable phenotypic expression, resulting from the genetic abnormality in the TSC1 gene on chromosome 9 coding for hamartin, and the TSC2 gene on chromosome 16 coding for tuberin. The majority of TSC patients suffer from epilepsy starting at young age and it is usually intractable with medication. Therefore, surgical treatment is considered early in the management of seizure in these patients. Literature and the surgical outcome at Severance Children's Hospital epilepsy clinic are reviewed for better management.

Methods: Patients medical record and imaging studies were reviewed from January 1, 2007 to December 312012 who underwent surgical treatment for epilepsy. Patient characteristics and surgical outcome were analyzed.

Results: 527 cases of epilepsy surgery were performed during the period and 16 patients were surgically managed for epilepsy with TSC. 9 were male and average age at surgery was 10.2 yrs. All presented with first seizure less than 1 year old. 2 VNS and 1 selective corpus callosotomy were performed. One patient underwent functional hemispherotomy and the rest underwent staged operation for resective surgery. One patient first undergone VNS and seizure recurred after a year and undergone resective surgery at the end. Most of the patient reached Engel classification 1A after surgery except the two patients undergone callosotomy and VNS alone. Conclusion: Surgical treatment should be considered at early stage during management of epilepsy in TSC patients. Identification and complete resection of epileptogenic tuber are the most important factor for good surgical outcome. VNS and callosotomy can be used as palliative measures but the seizure control is poor compared to respective surgery.

\section{3}

Outcomes of epilepsy surgery for glioneuronal tumours in childhood and adolescence: the timing of intervention

Georgia Ramantani ${ }^{1}$, Navah Ester Kadish ${ }^{2}$, Constantin Anastasopoulos ${ }^{3}$, Karl Strobl ${ }^{4}$, Angeliki Stathi ${ }^{1}$, Armin Brandt ${ }^{1}$, Hans Mayer ${ }^{4}$, Kathrin Wagner $^{1}$, Susanne Schubert-Bast ${ }^{2}$, Rudolf Korinthenberg ${ }^{3}$, Andreas Schulze-Bonhage ${ }^{1}$, Josef Zentner ${ }^{5}$, Vera van Velthoven ${ }^{5}$, Thomas Bast ${ }^{2,4}$ ${ }^{1}$ Epilepsy Centre, University Hospital Freiburg, Germany; ${ }^{2}$ Department of General Paediatrics, Children's University Hospital, Heidelberg, Germany; ${ }^{3}$ Division of Neuropaediatrics and Muscular Disorders, Department of Paediatrics and Adolescent Medicine, University Hospital Freiburg, Germany; ${ }^{4}$ Epilepsy Centre Kork, Kehl-Kork, Germany; ${ }^{5}$ Department of Neurosurgery, University Hospital Freiburg, Germany

Purpose: To investigate the seizure and cognitive outcomes of children and adolescents undergoing resective surgery for refractory epilepsy associated with glioneuronal tumours and to determine their predictive factors, with an emphasis on time-related variables.

Methods: We retrospectively analysed the findings of presurgical evaluation, resection types and outcomes over 1.3-12.3 years (mean 7.3) of 29 consecutive children and adolescents who underwent resection at the Epilepsy Centre Freiburg in 2000-2011. The mean age at epilepsy onset was 7.9 years (range $0-15.4$ ), the mean age at surgery was 11.7 years (range 2.6-17.3), and the mean epilepsy duration was 3.8 years (range 0.3-15.3). Aetiology comprised 13 dysembryoplastic neuroepithelial tumours and 16 gangliogliomas, with additional focal cortical dysplasia in 5 cases.

Results: $86 \%$ of children were seizure free 12 months after surgery and $76 \%$ remained seizure free at final follow-up; $62 \%$ discontinued antiepileptic drugs. Gross-total resection was related to significantly higher rates of seizure-freedom. Higher presurgical cognitive functioning (full scale IQ, verbal IQ) was related to shorter duration of epilepsy independent of age at epilepsy onset, thus determining postsurgical functioning. Improvements in verbal IQ, performance IQ and visual memory as well as a trend towards improvement in full scale IQ were established after surgery. No deterioration in any of the cognitive variables was noted on a group level.

Conclusion: With completeness of resection predisposing to favourable outcomes regarding seizure alleviation, a short latency to surgery appears crucial in preserving cognitive functions, thus supporting early surgical intervention.

124

Hemispherotomy: Aetiology, complications and long-term seizure outcome in paediatric patients

Marec von Lehe ${ }^{1}$, Robert Sassen ${ }^{2}$, Stefan Kuczaty ${ }^{2}$, Johannes Schramm ${ }^{3}$ ${ }^{1}$ University Hospital Bochum, Germany; ${ }^{2}$ Epileptology and ${ }^{3}$ Neurosurgery, University Hospital Bonn, Germany

Objective: In selected patients hemispheric deafferentiation is an effective option to treat epilepsy. We report our experience with a large group of 
children / adolescents with special attention to aetiology and long-term follow up.

Methods: Ninety-six patients between 4 months and 18 years of age (mean $7.3 ; 47$ male; 48 on the left) were analysed concerning presurgical work-up, surgical approaches, complications and follow-up. Appropriate seizure outcome data was available in 92 patients (followup 12-235 months, mean 99).

Results: In two patients incomplete disconnection was suspected from post-operative MRI, one patient was re-operated but not confirmed. Nine patients had post-operative complications (e. g. subdural hematoma, CSF fistula, $9 \%$ ) and 3 of them needed surgery (3\%). One patient died $(1 \%$ mortality). Six patients needed surgery for hydrocephalus / dilateted ventricle after 1-84 months ( $6 \%$; rate of new shunts: $5 \%), 4$ of them had porencephalic cysts $(67 \%)$. Eighty-five percent of patients were seizure free (ILAE class1, last available outcome (lao)) and long-term follow-up was stable after 4,8 and 12 years $(83 \%, 85 \%$ and $85 \%$ ILAE class1, respectively). Patients with porencephalic cysts had an excellent seizure outcome (95\% ILAE class1 (lao), N=42), with Rasmussen's encephalitis $79 \%(\mathrm{~N}=14)$ and with extensive dysplastic lesions $80 \%$ $(\mathrm{N}=10)$ were seizure free. In patients with hemimegaencephaly seizure outcome was less favourable $(60 \%, \mathrm{~N}=10)$.

Conclusion: Seizure outcome after functional hemispherotomy is promising and without "running down phenomenon" in the long run. Aetiology of epilepsy is a crucial factor for predicting seizure outcome and post-operative hydrocephalus.

\section{5}

Epilepsy surgery: Better outcome in children younger than 5 years of age explored by Stereo-EEG

Georg Dorfmuller ${ }^{1,2}$, Sarah Ferrand-Sorbets ${ }^{1}$, Mathilde Chipaux ${ }^{1}$, Christine Bulteau ${ }^{1,2}$, Olivier Delalande ${ }^{1}$, Martine Fohlen ${ }^{1}$, Delphine Taussig $^{1}$

${ }^{1}$ Department of Pediatric Neurosurgery, Fondation A. de Rothschild, Paris, France; ${ }^{2}$ Inserm U663, Descartes University, Paris, France

Introduction: We report on a surgical series of children with intractable epilepsy explored with invasive EEG to evaluate the clinical characteristics and post-operative outcome according to age.

Method: All children were explored with Stereo-EEG before surgery within the past 2 years at our institution. We compared children younger than 5 years of age (group $1 \mathrm{n}=21$ ) to those of 5 years and older (group $2 \mathrm{n}=44$ ). Results: In group 1, the mean age was 39 months ( 20 - 60 months). The extension of the exploration was comparable in both groups: with the insula considered as a separate lobe, at least three lobes were explored in all patients, up to four or five lobes in $76 \%$ in group 1 versus $70 \%$ in group 2. Surgery was rejected in two patients from group 1 and 12 from group 2. Comparing the underlying pathological substrate, we found cortical dyspasia type 2 in $57 \%$ in group 1 versus $42 \%$ in group 2, DNET in $9.5 \%$ versus $18.2 \%$, hippocampal sclerosis in $9.5 \%$ versus $6.1 \%$, and one patient with hypothalamic hamartoma in each group. With a mean post-operative follow-up of 16 months, 76\% were Engel 1 in group1, versus $59 \%$ in group 2 , and $14 \%$ were Engel $2 / 3$ in group 1, versus $15 \%$. Conclusion: Our results show that children younger than 5 years of age had a better post-operative outcome than the older children. Suitability of stereo-EEG and more frequent MRI abnormally in youngest patients allow a better guidance of the surgical planning.

\section{6}

Seizure outcomes of extratemporal epilepsy surgery in childhood: arguments for early intervention

Georgia Ramantani ${ }^{1}$, Constantin Anastasopoulos ${ }^{2}$, Armin Brandt ${ }^{1}$, Angeliki Stathi ${ }^{1}$, Karl Strobl ${ }^{3}$, Susanne Schubert-Bast ${ }^{4}$, Gert Wiegand ${ }^{5}$, Rudolf Korinthenberg ${ }^{2}$, Ulrich Stephani ${ }^{5}$, Andreas Schulze-Bonhage ${ }^{1}$, Josef Zentner ${ }^{6}$, Vera van Velthoven ${ }^{6}$, Thomas Bast ${ }^{3,4}$
${ }^{1}$ Epilepsy Centre, University Hospital Freiburg, Germany; ${ }^{2}$ Division of Neuropaediatrics and Muscular Disorders, Department of Paediatrics and Adolescent Medicine, University Hospital Freiburg, Germany; ${ }^{3}$ Epilepsy Centre Kork, Kehl-Kork, Germany; ${ }^{4}$ Department of General Paediatrics, Children's University Hospital, Heidelberg, Germany; ${ }^{5}$ Department of Neuropediatrics, University Medical Center Schleswig-Holstein, Kiel, Germany; ${ }^{6}$ Department of Neurosurgery, University Hospital Freiburg, Germany

Purpose: To investigate the long-term seizure outcomes of children and adolescents undergoing extratemporal resections for refractory epilepsy and determine their predictive factors.

Methods: We retrospectively analyzed the findings of presurgical evaluation, resections and outcome at mean 7 years (range 1-15.3) of 106 consecutive patients who underwent resection at the Epilepsy Center Freiburg in 1999-2012. The mean age at epilepsy onset was 4.6 years (range 0-15), the mean age at surgery was 10.4 years (range 1.3-18), and the mean epilepsy duration was 5.9 years (range 0.4-17.9). Aetiology comprised focal cortical dysplasia in $68(64 \%)$ and tumors in $27(25 \%)$ cases, tuberous sclerosis in $4 \%$ and scars in $7 \% .37$ (35\%) children underwent long-term intracranial EEG recordings and $41(39 \%)$ intraoperative electrocorticography.

Results: 68 (64\%) children were seizure free at last follow-up; 43 (41\%) discontinued antiepileptic drugs. The completeness of resection was the strongest predictor of postsurgical seizure arrest. Of presurgical factors, younger age at surgery, lower seizure frequency, and absence of secondary generalization were associated with significantly higher rates of seizure freedom. Of postsurgical factors, early seizures and epileptiform abnormalities in EEG were related to significantly higher rates of seizure recurrence. Conclusion: $64 \%$ of children with surgically treated extratemporal epilepsy had an excellent long-term outcome. With completeness of resection predisposing to favorable outcomes regarding seizure freedom, younger age at surgery and lower presurgical disease burden appear crucial, thus supporting early intervention. Early seizures and interictal epileptiform discharges in EEG are not benign, but constitute key markers of seizure recurrence.

127

Resection within Rolandic cortex in pediatric epilepsy surgery: Report of 7 cases

Hsin-Hung Chen, Chien Chen, Shih-Chieh Lin, Sheng-Che Hung, Ting-Rong Hsu, Kai-Ping Chang, Shang-Yeong Kwan, Donald M. Ho, Tai-Tong Wong, Yang-Hsin Shih

Taipei Veterans General Hospital National Taiwan University, Taipei, Taiwan

Purpose: In children with intractable epilepsy due to focal lesion, complete resection of the epileptogenic foci provides the best opportunity for seizure freedom. The goal becomes a dilemma when the lesion involves Rolandic cortex due to the risk of permanent neurological and functional deficits. We describe the results of 7 epilepsy patients younger than 18 years old with lesions in the Rolandic cortex. With careful case selection and a comprehensive epilepsy program, it is possible to resect eloquent cortex and preserve function.

Materials and methods: 6 patients were retrospectively reviewed from 2008 to 2013 in Taipei Veterans General Hospital, who had epileptogenic foci localized to the Rolandic cortex ( $\mathrm{M}: \mathrm{F}=4: 3$, mean age=9 y/o, range 218 years). Their medical records, MRI, functional MRI, positron emission topography, magnetoencephalogram, and tractography were reviewed. Epilepsy management goals were carefully determined by correlating seizure semiology, with findings obtained from electrophysiological studies and appropriate functional neuroimaging. Surgery utilized intraoperative electrocorticography and stimulation techniques to tailor the lesionectomy including in the Rolandic cortex. 
Results: 4 of 7 patients had an immediate transient hemiparesis following surgery. These all resolved at 3 months follow-up except fine-skill motor function. The pathology demonstrated $4 \mathrm{FCD}, 1$ polymicrogyria, 1 ependymoma, 1 DNET. All 7 patients achieved good epilepsy surgery outcome (Engel class I/II) with follow-up of up to 4 years.

Conclusion: Our experience demonstrates that with a careful perioperative epilepsy surgery work-up, appropriate application of functional neuroimaging, and a comprehensive epilepsy and rehabilitation program, it is possible to resection eloquent cortex and preserve function.

\section{8}

Risk and benefit of resective epilepsy surgery in the first years of life Caroline Meyer ${ }^{1}$, Christoph Schoene-Bake ${ }^{4}$, Robert Sassen ${ }^{2}$, Stefan Kuczaty $^{2}$, Marec von Lehe ${ }^{3}$

${ }^{1}$ Department of Neurosurgery, ${ }^{2}$ Department of Epileptology, University Hospital Bonn; ${ }^{3}$ Department of Neurosurgery, University Hospital Bochum; ${ }^{4}$ Department of Pediatrics, University Hospital Freiburg

Background and aims: Resective surgery in children is an established method to treat drug-resistant epilepsy. Our goal was to determine risk and benefit of this invasive treatment option in very young children.

Methods: We retrospectively analyzed data of 49 children who underwent surgery up to the age of 72 months. We excluded disconnective procedures (hemispherotomy, callosotomy). Long-term follow-up (FU) was assessed either by clinical records or a routine FU telephone questionnaire.

Results: Mean age at seizure onset was 19 months. Noninvasive EEG showed focal epileptic discharges $(69 \%)$, both generalized and focal etPs $(19 \%)$ and only generalized etPs (12\%). Invasive EEG recording was done in $26.5 \%$. Mean age at surgery was 45 months. Localization was extratemporal in $41.2 \%$, temporal in $37.7 \%$ and multilobar in $21.6 \%$. Most common histological findings were dysplasias (43\%) and tumors $(35 \%)$. The rate of new unexpected permanent deficits was $4 \%(n=2)$ with one hemiparesis and one hemianopia. One child had a calculated hemianopia, no mortality. After a mean FU of 7.7 years (range 1-23yrs) $73.5 \%$ were completely seizure free (ILAE 1) and $43 \%$ were without antiepileptic drugs. $12 \%$ showed marked improvement (ILAE 2-4). Presurgical generalized EEG was not associated with a worse outcome. There were no significant correlations between potentially predictive factors (age at operation, localization) and seizure outcome.

Conclusion: Despite the higher level of uncertainty in pre-surgical workup and presumably higher rate of peri-operative complications, epilepsysurgery in very young children is a safe and effective treatment option that renders approximately $3 / 4$ of patients seizure free.

129

Hemimegalencephaly: challenges and results of the surgical treatment

Enrico Loriatti ${ }^{2}$, Ana Hamad ${ }^{1}$, Ursula Thomé ${ }^{1}$, Ricardo S. Oliveira ${ }^{2}$, Marcelo Volpon ${ }^{2}$, Americo Sakamoto ${ }^{1}$, Helio R. Machado ${ }^{2}$

${ }^{1}$ Department of Neurology, ${ }^{2}$ Department of Pediatric Neurosurgery, Centre for Epilepsy Surgery, University of Sao Paulo, Ribeirao Preto, Brazil

Introduction: Hemimegalencephaly (HME) is a rare malformation of the cortical development arising from an abnormal proliferation of anomalous neuronal and glial cells, leading to hypertrophy of the whole affected cerebral hemisphere. The pathogenesis is still unknown. Patients with HME can present with early onset epileptic syndromes, which are often refractory to medical treatment and associated with impaired neurological development. Surgery is the most effective treatment to achieve seizure control, and it also provides good results on the psychomotor development when performed early. The surgical therapy of HME, however, is still burdened by high complication rates and mortality, and the best surgical technique is still unknown in literature.
Methods: Clinical data of 23 patients with the diagnosis of HME who underwent hemispherotomy or partial resections on a single center were reviewed.

Results: Mean age at surgery was 5,27 years; mean age at epilepsy onset was 37,7 weeks; mean seizure duration prior to surgery was 4,47 years; and mean follow-up was 7,3 years; postop worse defict were found in $6 /$ 23 patients (24\%). Seizure outcome was classified as Engel class I in11/ $23(47,8 \%)$ patients, Engel class II in 2/23 (8,7\%), Engel class III in 6/23 (26\%) of the patients, and Engel class IV in $2 / 23(8,7 \%)$; the mean days of hospitalization after the surgery was 18,34 days; the morbidity rate was $9 /$ 23 (39,1\%); and the mortality rate was $1 / 23(4,3 \%)$.

Conclusions: Hemispherotomy and partial surgery in HME can be safely performed in children with satisfactory seizure outcome and complication rates.

\section{0}

Reduction of epileptiform activity through local valproate-implants in a rat neocortical epilepsy model

Thomas M. Freiman ${ }^{1}$, Michael P. Rassner ${ }^{2}$, Jonas M. Hebel ${ }^{2}$, Dirk-Matthias Altenmueller ${ }^{3}$, Thomas J. Feuerstein ${ }^{2}$, Josef Zentner ${ }^{1}$

${ }^{1}$ Department of Neurosurgery, ${ }^{2}$ Section of Clinical Neuropharmacology, ${ }^{3}$ Section of Epileptology, Neurocentre, University Medical Centre, Albert-Ludwigs University, Freiburg, Germany

Pharmacological treatment of epilepsies is limited due to side effects of systemically of applied anticonvulsants and some of most efficient substances do not pas the blood-brain barrier. To overcome these limitations, we tested in an animal experimental model efficacy of local epilepsy therapy. Our results showed for the first time reduction of epileptiform activity through local application of valproate polymers. A novel experimental technique by inducing a neocortical epileptogenic focus in the M1 region of the rat was developed. Tetanus toxin was injected intracortically and cobalt (II) chloride $\left(\mathrm{CoCl}_{2}\right)$ was applied locally. Intracortical encephalography $(\mathrm{ECoG})$ electrodes were implanted in all rats and long-term video-ECoG recordings were performed. For local antiepileptic therapy biodegradable valproate-containing polymer matrices were implanted at the surface of the cortex above the focus. Antiepileptic effects were evaluated by reduction of clear-cut epileptiform potentials (EPs) per hour (h). Animals with triple $50 \mathrm{ng}$ tetanus toxin injections plus $10 \mathrm{mg} \mathrm{CoCl}_{2}$ application showed continuous spike-wave discharges. Saline-implanted animals showed a mean of $4.5 \mathrm{EPs} / \mathrm{h}$ in the first week, the EP frequency increased to $31.1 \mathrm{EPs} / \mathrm{h}$ in the second week. The VPA-implant animals showed a reduction in their EP frequency, amounting to $43.6 \mathrm{EPs} / \mathrm{h}$ in the first week and to $13.3 \mathrm{EPs} / \mathrm{h}$ in the second week. The increase of EPs/h in saline-implant animals $(26.4 \mathrm{EPs} / \mathrm{h})$ was significantly $(p=0.04)$ different from the decrease of EPs/h in VPAimplant animals (-40.1 EPs/h). Therefore, local therapy may be become an alternative treatment modality of epilepsies in future.

\section{1}

Outcome following pediatric temporal lobe epilepsy surgery - Does extent \& type of pathology matters?

Dattatraya Muzumdar ${ }^{1}$, Sangeeta Ravat ${ }^{2}$, Yogesh Ghodge ${ }^{2}$, Urvashi $\mathrm{Shah}^{2}$, Vivek Jain ${ }^{2}$, Nina Sawant ${ }^{3}$

Departments of ${ }^{1}$ Neurosurgery, ${ }^{2}$ Neurology and ${ }^{3}$ Psychiatry, Seth G.S.Medical College and King Edward VII Memorial Hospital, Parel, Mumbai, India

Introduction: Although temporal lobectomy is a well-established surgical procedure for temporal lobe epilepsy in adults, it is uncommon in children

Methods: The case records of 66 surgically treated children aged between 3-18 years with drug-resistant temporal lobe epilepsy at the Seth G.S.Medical College and King Edward VII Memorial hospital between 
2001-2011 were retrieved. Patients showing a discrete epileptogenic temporal lesion and ictal EEG demonstrating temporal lobe seizure origin in concordance with MRI brain were included. All patients underwent continuous non invasive video-EEG monitoring and MRI brain. Interictal and ictal SPECT was done in patients with bilateral lesion or ictal onset on EEG. Detailed neuropsychological evaluation was performed. The seizure outcome after temporal lobe surgery was evaluated.

Results: There were 24 females and 42 males. The mean age at surgery was 14.3 years. The mean duration of epilepsy was 6 years. All patients who had isolated mesial temporal sclerosis (MTS) or MTS with $\mathrm{CD}$ on imaging underwent anterior temporal lobectomy (ATL) with amygdalohippocampectomy. ATL was done in 4 patients. Lesionectomy was performed in 8 patients. The mean duration of follow-up was 3.5 years. 58 patients had unilateral lesion on MRI, and $50(86 \%)$ of these were in Engel's class I and $8(14 \%)$ patients were in Engel's class II. 8 patients had bitemporal lesion on MRI, 4 (50\%) of them were in Engel's class I (2 MTS\& 2 MTS with CD) and $4(50 \%)$ in Engel's class II (1 MTS \& 3 MTS with CD). Conclusion: Temporal lobe resections for epilepsy in children are effective and safe with a favorable impact on seizure control. Children with neuroimaging abnormalities have a significantly higher likelihood of medical intractability. MTS occurs frequently in association with CD in children.

\section{Neuro-oncology I}

\section{2}

Does radiotherapy-induced reduction in tumor volume translate into longer survival for children with diffuse intrinsic pontine gliomas?

Yasser Jeelani ${ }^{1}$, Stefan Bluml ${ }^{2}$, Matthew Borzage ${ }^{2}$, Stephanie Da Silva ${ }^{1}$, Mark Krieger ${ }^{1}$, J. Gordon McComb ${ }^{1}$

${ }^{1}$ Division of Neurosurgery, Children's Hospital Los Angeles, Department of Neurological Surgery, Keck School of Medicine, University of Southern California, Los Angeles, California; ${ }^{2}$ Department of Radiology, Children's Hospital Los Angeles, University of Southern California, Los Angeles, California

Introduction: Despite extensive trials, Diffuse Intrinsic Pontine Gliomas (DIPGs) carry a uniformly grim prognosis. While focal radiation therapy to the brainstem remains the mainstay of treatment, a definitive relationship between radiation-induced tumor size reduction and enhanced survival is not yet objectively established.

Methods: Under an IRB approval, a retrospective analysis of all patients with a diagnosis of DIPG primarily treated with focal radiation within the last 10 years. A neuro-radiologist and a neurosurgical resident independently mapped pre- \& post-irradiated tumor volumes using Fast Spin Echo (FSE) acquired T2-weighted MR images.

Results: Twenty-one patients (12 female) qualified; median age at diagnosis was 86 months (range: 41-152 months). All received primary radiation: 30-33 fractions of Intensity modulated radiotherapy (IMRT) ranging between 4500-6000 cGy administered over mean duration of 39.5 days (range: $38-42 \mathrm{~d}$ ). Concomitant chemotherapy was instituted in 12 patients. Post irradiation scans were obtained after a mean interval of 5 weeks (range: $3 \mathrm{~d}-18$ weeks). The median survival was 10.8 months (range: 3.7-27.6 months). There was a net reduction in volume in 19 patients and an interval increase in size in 2 . In the former, the median percentage reduction was $57.9 \%$ (SD 27.9) whereas the overall median volumetric reduction was $12.1 \mathrm{~cm}^{3}$ (range: -42.8 to $+1.32 \mathrm{~cm}^{3}$ ). Statistically, $>50 \%$ reduction in tumor volume was associated with a significantly improved survival $(p=0.04)$. Net reduction in tumor volume, however, failed to predict better outcome $(\mathrm{p}=0.07)$.
Conclusions: A radiation-induced reduction in tumor volume by a factor greater than $50 \%$ may modestly prolong survival in children with DIPG \& hence may aid in prognostication and monitoring therapy.

\section{3}

\section{Intraoperative ultrasound in pediatric brain tumors: Does} the surgeon get it right?

Ash Singhal, A. Ross Hengel

British Columbia Children's Hospital, Division of Pediatric Neurosurgery, Vancouver, Canada

Introduction: Intraoperative ultrasound (IOUS) is a valuable tool - inexpensive, adds minimal surgical time, and involves minimal risk. The diagnostic predictive value of IOUS is not fully characterized in Pediatric Neurosurgery. Our objective is to determine if surgeon-completed IOUS has good concordance with post-op MRI in estimating extent of surgical resection (EOR) of pediatric brain tumors.

Methods: We reviewed charts of pediatric brain tumor resections (single institution 2006-2013), including those with IOUS and immediate postoperative imaging ( $<1$ week). The surgeon's estimation of the EOR based on IOUS and the post-operative neuroimaging results (gold standard) were collected, as well as information about the patients/tumors.

Results: 57 cases were reviewed. The concordance of interpretation, between IOUS and post-op MR was $89.3 \%$. Of 41 cases where IOUS suggested gross total resection, 35 were confirmed on MR (negative predictive value, NPV: $85 \%$ ). All 15 cases where IOUS suggested subtotal resection were confirmed on MR (positive predictive value, PPV: $100 \%$ ). IOUS had a $94 \%$ concordance with postoperative imaging in 33 infratentorial tumors and there was $83 \%$ concordance in 24 patients with supratentorial tumors.

Conclusion: The results from this study suggest that IOUS is reliable with residual tumor (PPV - 100\%) but less so when it suggests no residual (NPV $-85 \%$ ). These predictive values of IOUS suggest there is room for improvement in intra-operative tumor detection, either by improving the diagnostic validity of ultrasound, or by the addition of other intraoperative adjuncts.

\section{4}

miR-92b inhibitor promoted glioma cell apoptosis by blocking the $\mathrm{Wnt} / \beta$-catenin signaling pathway in vitro

Qifeng Li, Yang Zhao, Xiaoguang He, Chenkai Ma, Jie Ma

Department of Pediatric Neurosurgery, Xinhua Hospital, Shanghai Jiao Tong University, School of Medicine

Previous studies have shown that miR-92b is overexpressed in brain primary tumor, as compared to primary tumors from other tissues and their metastases to the brain, However, there is no report concerning the relationship between miR-92b and the $\mathrm{Wnt} / \beta$-catenin signaling pathway in glioma. Here, we identified that miR-92b was overexpressed in human glioma tissues compared with their corresponding nontumorous tissues by miRNA array analysis. The results got confirmed in four glioma cell lines. We also found out that miR-92b disruption could significantly promote apoptosis and inhibit the proliferation of glioma cells in vitro. Target prediction analysis and dual luciferase report assay confirmed that the inhibitory protein Dickkopf-3 gene (DKK3) was a direct target of miR-92b. In glioma cells, RNA interference silencing of DKK 3 proved that down regulated miR-92b regulated constitutive inhibition of $\beta$-catenin through targeting DKK3. We also proved that miR$92 \mathrm{~b}$ could regulate the expression of downstream genes of the $\mathrm{Wnt} / \beta$ catenin signaling pathway, such as Bcl2, c-myc, and c-jun in glioma cells. In a nutshell, our findings demonstrated a new regulatory mechanism of miR-92b in controlling glioma apoptosis. Targeting miR-92b might provide a strategy for the management of glioma. 


\section{5}

Simultaneous segregation of tumor-spheres and mesenchymal stem-like cells from primitive neuroectodermal tumor specimen Seok-Gu Kang ${ }^{1}$, Jiyong Kwak ${ }^{2}$, Hye-Jin $\mathrm{Shin}^{2}$, Se-Hoon $\mathrm{Kim}^{3}$, Jin-Kyoung Shim ${ }^{1}$, Ji Hyun Lee ${ }^{1}$, Yong-Min Huh ${ }^{4}$, Eun-Kyung Park ${ }^{1}$, Yong-Kil Hong ${ }^{5}$, Su Jae Lee ${ }^{6}$, Dong-Seok Kim ${ }^{1}$

${ }^{1}$ Department of Neurosurgery, ${ }^{2}$ Department of Medical Science, ${ }^{3}$ Department of Pathology, ${ }^{4}$ Department of Radiology, Severance Hospital, Yonsei University College of Medicine, Seoul, Korea ${ }^{5}$ Department of Neurosurgery, Seoul St. Mary's Hospital, The Catholic University of Korea College of Medicine, Seoul, Korea ${ }^{6}$ Department of Chemistry, Hanyang University, Seoul, Korea

It has been reported that cancer stem cells (CSCs) can be isolated from primitive neuroectodermal tumor (PNET) specimens. Moreover, mesenchymal stem-like cells (MSLCs) were isolated from Korean glioma specimens. Here, we tested whether tumor-spheres and MSLCs can be simultaneously isolated from a single PNET specimen, a question that has not been addressed. We isolated single-cell suspensions from PNET specimens, then cultured these cells using methods for MSLCs or CSCs. Cultured cells were analyzed for surface markers of CSCs using immunocytochemistry and for surface markers of bone marrow-derived mesenchymal stem cells (BM-MSCs) using fluorescence-activated cell sorting (FACS). Tumor-spheres were exposed to neural differentiation conditions, and MSLCs were exposed to mesenchymal differentiation conditions. Possible locations of MSLCs within PNET specimens were determined by immunofluorescence analysis of tumor sections. Cells similar to tumor-spheres and MSLCs were independently isolated from one of two PNET specimens. Spheroid cells, termed PNET-spheres, were positive for CD133, nestin, and negative for musashi, podoplanin. PNET-spheres were capable of differentiation into immature neural cells and astrocytes, but not oligodendrocytes or mature neural cells. FACS analysis revealed that adherent cells isolated from the same PNET specimen, termed PNETMSLCs, had surface markers similar to BM-MSCs. These cells were capable of mesenchymal differentiation. Immunofluorescence labeling indicated that some $\mathrm{CD} 105^{+}$cells might be closely related to endothelial cells and pericytes. We showed that both tumor-spheres and MSLCs can be isolated from the same PNET specimen. PNET-MSLCs occupied a niche in the vicinity of the vasculature and could be a source of stroma for PNETs.

\section{6}

Tumor suppressive microRNAs in pediatric malignant gliomas Muh-Lii Liang ${ }^{1,2}$, Ya-Ni Tsai ${ }^{3}$, Kim-Hai Ng ${ }^{3}$, Wei-Chung Cheng ${ }^{3}$, Tai-Tong Wong ${ }^{4}$, Hsei-Wei Wang ${ }^{2,3}$

${ }^{1}$ Division of Pediatric Neurosurgery, Department of Neurosurgery, Neurological Institute, Taipei Veterans General Hospital, R.O.C.; ${ }^{2}$ Institute of Clinical Medicine, National Yang-Ming University; ${ }^{3}$ Institute of Microbiology and Immunology, National Yang-Ming University; ${ }^{4}$ Department of Surgery, Cheng Hsin General Hospital

Pediatric gliomas rank one of the common malignancies among childhood cancer, and high-grade gliomas are the leading cause of cancerrelated mortality in the pediatric group. Treatment of these tumors remains a challenge despite aggressive multimodal therapy with surgical resection, radiation and chemotherapy. In order to explore the innovative therapeutic targets, we analyzed 12 cases of low- and high-grade pediatric gliomas through the advanced small RNA sequencing (smRNA-seq) application of the next-generation sequencing (NGS) technology. The significant up-regulated microRNAs in pediatric high-grade gliomas were miR96-5p, miR182, miR183, miR561-5p and miR4746; and downregulated microRNAs, like miR-31-5p, miR34a-3p, miR34a-5p, miR137, miR221-3p, miR221-5p, miR222-3p and miR6500. Reduced expression of miR-137 and miR-6500 in high-grade glioma tissues were verified by using RT-qPCR in 28 pediatric gliomas samples, implying their tumor suppressive role. Over-expressing miR-137 showed suppression of proliferation in pediatric gliomas cell lines UW479 and SF188. More importantly, growth suppression could be further enhanced by combining miR-137 over-expression and temozolomide chemotherapy. Further study will have the potential to develop novel chemo-sensitizer therapeutic models for these highly malignant pediatric tumors.

\section{7}

Meningiomas in childhood: a report of 21 consecutive cases and review of the literature

Friederike S. Fritzsche, Gertrude Kammler, Manfred Westphal, Pedram Emami

University Medical Center Hamburg-Eppendorf, Department of Neurological Surgery, Hamburg, Germany

Objective: Meningiomas in children and adolescents are a rare condition and still poorly investigated clinical entity. The purpose was to determine prognostic variables.

Methods: 21 patients with age between 0 and 17 years underwent surgery for central nervous system meningioma at our clinical centre during a period of 1981-2010. Clinical data (such as age, sex, tumour location, presence of NF2 and histopathology) was analyzed retrospectively. A review of literature in pubmed, cochrane and medline was base for additional specific discussion.

Results: The group included 9 females and 12 males (mean age 10,7 years at surgery). The tumour localization showed broad variety (orbita, spinal cord, ventricle, convexity, skull base). Five children had evidence of NF2 $(23 \%)$. The histopathology revealed seventeen grade I meningiomas (81\%) and four grade II $(19 \%)$ meningiomas. The group of grade II meningiomas consisted of one female and three male patients, two with an associated NF2. In the male patient group existed four cases of transitional subtype of meningioma $(33,3 \%)$, none in the female population. Five tumours recurred, none was associated with NF2. Two of them were atypical subtype.

Conclusions: In contrast to the adult population male predominance is shown in this series. Recurrences were not associated with NF2 while the current literature classifies these patients as special risk category needing close follow-up. Surgery is the first line treatment and regardless of tumour localization the extent of tumour resection is an essential prognostic factor in meningiomas also in the paediatric age group.

\section{8}

Surgical management of subependymal giant cell astrocytomas in Tuberous Sclerosis Complex: indications and outcome Georg Dorfmüller ${ }^{1,2}$, Martine Fohlen ${ }^{1}$, Sarah Ferrand-Sorbets ${ }^{1}$, Christine Bulteau $^{1,2}$, Jessica Ternier ${ }^{1}$, Olivier Delalande ${ }^{1}$

${ }^{1}$ Pediatric Neurosurgery Department, Fondation A. de Rothschild, Paris, France; ${ }^{2}$ Inserm U663, Descartes University, Paris, France

Introduction: Subependymal giant cell astrocytomas (SEGA) are lowgrade glial tumors that develops in $10-15 \%$ of patients with Tuberous Sclerosis Complex (Bourneville disease), intraventricular, located near the foramen of Monro, often cause hydrocephalus and are potentially accessible to surgical treatment.

Methods: A retrospective series of patients who underwent surgical treatment for SEGA between 2000 and 2012 in our department. We reviewed the indications, the pre-operative clinical and radiologic details, the surgical management and the clinical and radiological follow-up.

Results: Seventeen patients (10 girls) were operated at an average age of 12.5 years. The surgical decision was decided on clinical signs in 7 patients and on radiological evolution in 10 patients (asymptomatic ventricular dilatation $\mathrm{n}=9$, or increase size of SEGA $\mathrm{n}=1$ ). Six patients had bilateral localization. Surgical 
treatment consisted on a trans-frontal trans-ventricular approach (16 patients) or endoscopic approach (1 patient), an external ventricular shunt was systematic. A ventriculoperitoneal shunt was necessary in 6 patients, all had a pre-operative hydrocephalus. Morbidity reached $17.6 \%$ (Korsakoff's syndrome $\mathrm{n}=1$, meningitis: $\mathrm{n}=2$ ). Resection was complete in 14 patients and incomplete in $17.6 \%$ of cases $(n=3)$. Further resection was performed in 3 cases. The mean duration of post-operative follow-up was 7.2 years. No recurrence was observed. There was no mortality. Conclusion: Surgery is associated with low morbidity and is an effective treatment of SEGA in children. It seems that early surgery (before ventricular dilatation) would limit the indications of ventriculoperitoneal shunt, thus justifying a close radiological monitoring.

\section{9}

Secondary dissemination of medulloblastomas in children Sara Ghayouri ${ }^{1}$, Yasser Jeelani ${ }^{2}$, Stephanie Da Silva ${ }^{2}$, J. Gordon McComb $^{2}$, Mark D. Krieger ${ }^{2}$

${ }^{1}$ Florida State University College of Medicine, Tallahassee, Florida; ${ }^{2}$ Division of Neurosurgery, Children's Hospital of Los Angeles, Department of Neurological Surgery, Keck School of Medicine, University of Southern California, Los Angeles, CA

Introduction: One-third of medulloblastomas disseminate over the course of the disease and carry an extremely poor prognosis. The risk factors for and patterns of dissemination have not been well described to date.

Methods: Under IRB approval, a retrospective analysis was performed on all patients with a histological diagnosis of medulloblastoma over a 10 year period in whom there was complete clinical and radiographic follow-up at a single institution. Dissemination patterns were analyzed.

Results: Fifty-seven patients ( 33 male) were identified. The mean age at diagnosis was 7.5 years (range $3 \mathrm{~m}-16.8 \mathrm{y}$ ). Median follow-up was 3.7 years (range 0-10y). All were treated under standard protocols. Six had disseminated disease at presentation; 1 was under 3 years of age. 21 had dissemination during their disease course; 3 were under age 3. 13/21 (61\%) of children with secondary dissemination had gross total resection of their tumors initially. Survival after secondary dissemination was on average 13.7 months (range 0.4-69m). Age, histology, location, and size of tumor did not impact the rate of secondary dissemination. Secondary dissemination did not independently impact survival in children under 3 whose survival was reduced by virtue of age alone, but was associated with a worse outcome in children over 3 years of age $(\mathrm{p}=0.04)$.

Conclusion: Secondary dissemination of medulloblastomas is relatively common, and can occur even when there is gross total resection of the tumor on presentation. Secondary dissemination was shown to convey a worse prognosis in standard risk patients (i.e., older children).

140

Does the presence or development of hydrocephalus affect the prognosis for diffuse intrinsic pontine glioma?

Ifeanyi Nwokeabia ${ }^{1}$, John Grimm ${ }^{2}$, Ira Bowen ${ }^{2}$, Yasser Jeelani ${ }^{2}$, Sara Ghayouri $^{2}$, Stephanie Da Silva ${ }^{2}$, J. Gordon McComb ${ }^{2}$, Mark D. Krieger ${ }^{2}$ ${ }^{1}$ Howard University College of Medicine, Washington, DC; ${ }^{2}$ Division of Neurosurgery, Children's Hospital of Los Angeles, Los Angeles, CA Department of Neurological Surgery, Keck School of Medicine, University of Southern California, Los Angeles, CA

Introduction: Diffuse Intrinsic Pontine Gliomas (DIPG) are high-grade tumors that are locally infiltrative, and have a uniformly poor prognosis despite available therapy. Development of hydrocephalus in patients with DIPG is common but little research exists correlating the incidence and progression of hydrocephalus to general outcome in these children.
Methods: In this IRB-approved study, 41 children with DIPG from 2000 to 2011 were retrospectively reviewed. Serial MRIs and CTs were evaluated for disease course and presence of hydrocephalus.

Results: The mean age was 8.1 years (range 0.3 mo-22.8 years) and 26 $(61 \%)$ were female. The mean overall survival (OS) of the group was 11.3 months. Children under 3 years of age had shorter average survival than older children (5.2 vs 12 months, $\mathrm{p}=0.05$ ). Eight patients received radiotherapy alone, 1 was treated with chemotherapy alone and 25 were treated with both radiotherapy and chemotherapy. 23 patients (56\%) had hydrocephalus; 9 at presentation and 14 during or after therapy. The mean OS for patients with hydrocephalus at presentation was 13.7 months vs. 11.8 months for patients without hydrocephalus at presentation $(\mathrm{p}=0.52)$. Patients who subsequently developed hydrocephalus had a mean survival of 6.7 months from the time of diagnosis of hydrocephalus and an overall survival of 11.8 months that was not significantly different from those without hydrocephalus $(\mathrm{p}=0.08)$.

Conclusion: The presence of hydrocephalus at the time of diagnosis of DIPG and during or after treatment did not affect prognosis in this series. Substantial survival is possible after the presentation of hydrocephalus. This information should be useful in counseling families.

\section{1}

Does the post-operative presence of an enhancing cyst wall in pilocytic astrocytoma indicate higher risk of tumor recurrence? Christine Bui ${ }^{1}$, Yasser Jeelani ${ }^{2}$, John Grimm ${ }^{2}$, Andrew Yousef ${ }^{2}$, Stephanie Da Silva ${ }^{2}$, J. Gordon $\mathrm{McComb}^{2}$, Mark D. Krieger ${ }^{2}$

${ }^{1}$ Perelman School of Medicine, University of Pennsylvania, Philadelphia, PA; ${ }^{2}$ Division of Neurosurgery, Children's Hospital of Los Angeles, Los Angeles, CA Department of Neurological Surgery, Keck School of Medicine, University of Southern California, Los Angeles, CA

Introduction: Is there a need to resect enhancing cyst walls in children with a pilocytic astrocytoma (PA)? This study investigates whether post-operative residual enhancing cyst walls in PAs are associated with a worse prognosis. Methods: An IRB-approved retrospective analysis was performed on children treated for cystic PA at a single institution between 2000 and 2012. Both pre- and post-operative MRIs were analyzed for the presence of an enhancing cyst wall.

Results: 51 children (27 male) presented with cystic PA (10 supratentorial; 41 infratentorial). The median age at diagnosis was 8.8 years (range: $2.7-$ 16.9). Mean follow-up was over 4 years (range: 1month-10years). 31 patients presented with enhancing cyst walls; postoperatively, 13/31(42\%) had residual enhancing cyst wall. Overall, 13/51 patients had tumor progression/recurrence. In 12/13 cases, progression occurred at the site of gross residual tumor. 7 patients with gross total resection of the solid tumor and postoperative enhancing cyst walls were followed for an average of over 3 years without tumor recurrence. Using Fisher's exact test, there was no statistically significant relationship between the presence of an enhancing post-op cyst wall and tumor recurrence or progression $(\mathrm{p}=1)$.

Conclusions: Residual cyst wall is not a risk factor for tumor recurrence. The goal of surgery should be to resect the solid tumor. There is no need to surgically resect the full extent of the enhancing cyst wall.

\section{2}

\section{Fractionated stereotactic radiosurgery is efficacious} and well-tolerated in the treatment of pediatric intracranial neoplasms

Todd C. Hankinson ${ }^{1}$, S. Reed Plimpton ${ }^{2}$, Nicholas K. Foreman ${ }^{3}$, Michael H. Handler ${ }^{1}$, Arthur K. Liu ${ }^{2}$

${ }^{1}$ Division of Pediatric Neurosurgery, Children's Hospital Colorado; ${ }^{2}$ Department of Radiation Oncology, University of Colorado, Denver; ${ }^{3}$ Division of Neuro-oncology, Children's Hospital Colorado

Introduction: While data in the adult population indicates that fractionated stereotactic radiosurgery (fSRS) is associated with high degrees of 
efficacy and tolerability, there is little published experience dedicated to the treatment of children using fSRS.

Methods: We retrospectively reviewed the experience at Children's Hospital Colorado/University of Colorado Denver in treating 24 lesions in 15 pediatric patients (age 2.6-24.9y, median 10.0y) using fSRS between 2007-2012.

Results: Patients received a median of 24Gy (range 20-25Gy) in 3 fractions (range 3-5). Two patients, one with a multiply recurrent astroblastoma and one with an Ewing's Sarcoma metastasis, had not received previous radiation therapy. Twenty-three lesions $(95.8 \%)$ were WHO Grade III-IV, with the exception being a pilomyxoid astrocytoma. Median tumor volume was $2.7 \mathrm{cc}(0.4-94.5 \mathrm{cc})$. Median follow-up was 12.5 months (range 1.0-47.1 months). The local control rate was $91 \%$ at 2 years and overall survival was $76 \%$ at 2 years post-fSRS. Radiation necrosis was seen at 7 of the treated sites $(29.2 \%)$ with a median onset time of 4.1 months (range 1.4-8.4 months).

Conclusions: ISRS is an effective and well-tolerated treatment for multiple types of intracranial pediatric neoplasms. Radiation necrosis may be more common than in the adult population.

\section{3}

Radiation-associated meningiomas in children: Clinical, pathological and cytogenetic characteristics

Samer K. Elbabaa ${ }^{1}$, Murat Gokden ${ }^{2}$, John R. Crawford ${ }^{3}$, Santosh Kesari ${ }^{3}$, Ali G. Saad ${ }^{2}$

${ }^{1}$ Department of Neurosurgery, Saint Louis University School of Medicine, Saint Louis, MO, USA; ${ }^{2}$ Department of Pathology, University of Arkansas for Medical Sciences and Arkansas Children's Hospital, Little Rock, AR, USA; ${ }^{3}$ Department of Neurosciences and Neuro-oncology Program at Moores Cancer Center, UC San Diego, La Jolla, CA, USA

Introduction: Radiation-associated meningiomas (RAMs) arise after treatment with radiation to the cranium and are recognized as clinically separate from sporadic meningiomas. Limited information exists about the clinical, pathological, and cytogenetic features of RAMs in pediatric patients.

Material and Method: Medical files were searched for patients who demonstrated meningiomas after a history of radiation to the brain. Only those patients in whom a meningioma occurred before the age of 18 years were included in this study. Clinical and demographic data along with the MIB-1 labeling index and cytogenetic studies were evaluated.

Results: M:F ration was 5:4 males. Median age of 5 years at radiation therapy. Latency period was a median of 10 years after radiation therapy (range 6-13 years). MIB-1 labeling index was a median of $6.6 \%$. There was no statistical difference in the MIB-1 LI between patients in whom the tumor recurred versus those who were disease free $(p=0.29)$. Five patients (55.6\%) displayed multiple meningiomas at the first presentation. Histological types included 1 clear cell meningioma, 2 fibroblastic meningiomas, 2 chordoid meningiomas, 7 meningothelial meningiomas (atypical in 2 cases), 1 xanthomatous meningioma and 1 chordoid meningioma. Cytogenetic studies showed that the loss of 22q12.2 was the most common abnormality followed by complex cytogenetic abnormalities, rearrangements between chromosomes 1 and 12 and $1 \mathrm{p}$ deletion. Conclusions: RAMs occurring in pediatric patients show an increased incidence of multiplicity on first presentation and unusual histological variants. There was no difference in the MIB-1 labeling index in children with RAMs as compared with that in children with non-RAMs.

\section{4}

The use of 5-aminolevulinic acid fluorescence guidance in resection of pediatric brain tumors

Matthias Preu $\beta^{1,7}$, Hans-Christof Renner ${ }^{1}$, Wolfgang Krupp ${ }^{1}$, Holger Christiansen $^{2}$, Lars Fischer ${ }^{2}$, Andrea Merkenschlager ${ }^{3}$, Wieland Kieß ${ }^{4}$, Wolf Müller $^{5}$, Norbert Manzo ${ }^{6}$, Jürgen Meixensberger ${ }^{1}$, Ulf Nestler ${ }^{1,6,7}$
${ }^{1}$ Department of Neurosurgery, ${ }^{2}$ Division of Pediatric Oncology and Haematology, ${ }^{3}$ Division of Neuropediatrics, ${ }^{4}$ Department of Children's and Adolescence Health, ${ }^{5}$ Insitute of Neuropathology, University Clinic Leipzig, Germany; ${ }^{6}$ Department of Neurosurgery, CHU Fort-de-France, Martinique, France; ${ }^{7}$ Department of Neurosurgery, Justus Liebig University, Giessen, Germany

Whereas in the adult population 5-Ala fluorescence guidance has been widely accepted for improving the extent of tumor resection, the application in children remains an off-label use. Even though most pediatric study protocols require a complete resection for improving outcome parameters, only few pediatric patients have been operated with fluorescence guidance and it remains questionable, whether and which pediatric tumors show useful fluorescence. We present casuistic reports of application of 5-Ala in children, collected from three different neurosurgical departments.

Patients and Methods: In children with suspicion of malignant intracerebral tumor, individual informed consent was obtained in each case from the parents. 5-Ala was administered according to the adult protocol with $20 \mathrm{mg} / \mathrm{kg}$ two hours before induction of anesthesia. We retrospectively analysed the records of 19 patients (14 male : 5 female, age 1-18 years), using the intraoperative neurosurgical protocol, the postoperative MRI results and the follow-up clinical examination.

Results: The use of 5-Ala fluorescence guidance proved to be safe and reliable in the pediatric patients. Fluorescence guidance was most useful for recurrent glioblastoma resection. Medulloblastoma tissue displayed fluorescence only inconsistently and pilocytic astrocytoma remained without staining. Ganglioglioma showed partial staining in the central tumor areas without allowing the use for circumferent resection.

Conclusion: 5-Ala fluorescence guidance in pediatric patients is most useful in recurrent high-grade gliomas.

\section{5}

Disseminated oligodendroglial-like leptomeningeal

tumours - diagnostic and therapeutic strategies

Matthias Preuss ${ }^{1 *}$, Ulf Nestler ${ }^{1}$, Holger Christiansen ${ }^{2}$, Andrea Merkenschlager $^{3}$, Wolfgang Hirsch ${ }^{4}$, Christian Geyer $^{5}$, Wieland Kiess ${ }^{6}$, Wolf Müller ${ }^{7}$, Stefanie Kästner ${ }^{8 *}$, Andreas Henssler ${ }^{9}$, A. Pekrun ${ }^{10}, \mathrm{H}$. Hauch $^{11}$, Martin U. Schuhmann ${ }^{12}$, Jürgen Meixensberger ${ }^{1}$, J. Pietsch ${ }^{13}$, Klaus Kuchelmeister ${ }^{13}$

${ }^{1}$ Department of Neurosurgery, ${ }^{2}$ Department of Pediatric Oncology and Haematology, ${ }^{3}$ Department of Neuropediatrics, ${ }^{4}$ Department of Pediatric Radiology, ${ }^{5}$ Department of Pediatric Surgery, ${ }^{6}$ Department of Children's and Adolescence Health, ${ }^{7}$ Department of Neuropathology University Leipzig, Germany; ${ }^{8}$ Department of Neurosurgery, Klinikum Nordhessen GmbH, Kassel, Germany; ${ }^{9}$ Department of Neurosurgery, Klinikum Bremen-Mitte, Bremen, Germany; ${ }^{10}$ Department of Pediatric Hematology and Oncology, Klinikum Bremen-Mitte, Bremen, Germany; ${ }^{11}$ Department of Pediatric Oncology, University Giessen, Giessen, Germany; ${ }^{12}$ Department of Neurosurgery, Karls-Universität Tübingen, Tübingen, Germany; ${ }^{13}$ Department of Neuropathology, University Bonn, Germany

*Authors contributed equally to this publication

During the past years, repeatedly reports came across central nervous system tumours with diffuse leptomeningeal spread in absence of a distinct primary mass lesion. Neuropathologists discussed new entities such as diffuse leptomeningeal glioneural tumour (DLGT) or disseminated oligodendoglial-like leptomeningeal tumour (DOGLT) without concludent definition of a new entity. A broad range of histopathological findings has been described so far in patients suffering from tumours with diffuse leptomeningeal spread. The term Disseminated Oligodendrogliallike Leptomeningeal Tumours (DOGLT) is suggested for that group of similar histopathological entities with distinct clinical features. However, these very rare tumours provide major difficulties in diagnostic strategies 
and therapy. We suggest a first diagnostic algorithm and radiological criteria as well as a treatment strategy after observation of seven clinical cases. Prognosis and clinical courses remain to be observed.

146

Effect of surgical debulking on post-operative cerebellar mutism Anthony Myint ${ }^{1}$, Yasser Jeelani ${ }^{1}$, Jessica Ashford ${ }^{2}$, Stephanie Da Silva ${ }^{1}$, J. Gordon McComb ${ }^{1}$, Mark D. Krieger ${ }^{1}$

${ }^{1}$ Division of Neurosurgery, Children's Hospital of Los Angeles, Los Angeles, CA Department of Neurological Surgery, Keck School of Medicine, University of Southern California, Los Angeles, CA; ${ }^{2}$ Division of Speech and Language Pathology, Doernbecher Children's Hospital, Oregon State Health University, Portland, OR

Introduction: Cerebellar mutism syndrome (CMS) is an established complication of posterior fossa surgery in children. Our study investigates possible risk factors associated with CMS.

Methods: An IRB-approved retrospective review was performed on all patients who underwent posterior fossa tumor resection at our institution between 2005 and 2012. Patient charts, radiographic studies, and patientevaluations by an onsite speech pathologist were reviewed. Patients younger than 2 years or with significant premorbid neurological deficits were excluded from the study due to difficulty in assessing speech.

Results: Of the 86 patients meeting the above criteria, 12 (14\%) had postoperative mutism. Mean age at time of operation was 8 years (range 2.518.8). Using chi-square independence testing, the following risk-factors were not found to be significantly associated with mutism: pre-operative hydrocephalus, duration of symptoms prior to surgery, choice of tumordebulking method, vermian tumor location, tumor type, and prolonged post-operative intubation. Ethnicity showed borderline significance $(\mathrm{p}=0.052$ ). In contrast, age in the 5 to 10 year range, tumor location in the right cerebellar hemisphere, and invasive tumor (necessitating subtotal resection) were found to be significantly associated with CMS. The mean pre-operative tumor size in those with and without cerebellar mutism was $3.95 \pm 0.86 \mathrm{~cm}$ and $4.22 \pm 1.33 \mathrm{~cm}$, respectively.

Conclusions: Our study suggests that age of 5 to 10 years at time of surgery, tumor location in the right cerebellar hemisphere, and invasive tumor (necessitating subtotal resection) all increase risk of post-operative mutism. Our results did not show a correlation between histology, vermian involvement, and resection technique in the development of CMS.

\section{7}

Multi-variate analysis of risk factors involved with a significant reduction in cerebellar Mutism after resection of posterior fossa medulloblastoma

Alan Siu, Elizabeth Wells, Tiffani Defreitas, John Myseros, Suresh Magge, Chima Oluigbo, Roger Packer, Robert Keating

Departments of Neurosurgery and Neurology, Children's National Medical Center, George Washington University, School of Medicine, Washington, DC, USA

Introduction: Cerebellar Mutism Syndrome(CMS) remains a challenging post-operative complication following resection of posterior fossa tumors(particularly medulloblastoma). Previous studies have alluded to associations with transvermian approach and CMS. We previously demonstrated a $39 \%$ incidence of CMS and subsequently adopted changes to reduce the incidence. We sought to systematically characterize the risk of developing CMS with resection of medulloblastoma.

Methods: A retrospective review of children with posterior fossa medulloblastoma was undertaken. Independent variables included: demographics, tumor size, surgical approach, CUSA usage, and infiltration of the vermis, brainstem and cerebellum. Our previously reported cohort was compared to the newer cohort. Wilcoxon rank sums/Fisher's exact tests were used for univariate analyses $(\mathrm{p}<0.05)$ and multivariate logistic regression.
Results: 65 children with posterior fossa medulloblastomas were identified. Compared to the previous cohort, the incidence of CMS significantly declined from $39 \rightarrow 13.5 \%$, with the implementation of the telovelar approach (vermis-sparing), avoidance of the CUSA and minimizing retraction. There were no cases of CMS with non-transvermian approaches $(\mathrm{p}<0.01)$. Other significant variables were CUSA usage, vermian and cerebellar infiltration. In the combined univariate/ multivariate analyses, the transvermian approach, tumor size and presence of brainstem infiltration were associated with CMS $(\mathrm{p}<0.05)$.

Conclusions: A significant reduction in our rate of CMS occurred(over past 8 years) for medulloblastoma patients. Our data suggests the use of vermis-sparing approaches, CUSA avoidance and less retraction as significant contributors to the reduction of this challenging post-operative complication.

\section{8}

Splitting of the cerebellar vermis in juvenile rats - effects on social behaviour, vocalization and motor activity

Elvis J. Hermann, Shadi Al-Afif, Mareike Staden, Kerstin Schwabe, Joachim K. Krauss

Department of Neurosurgery, Medical School Hannover, Hannover, Germany

Background: Radical resection of malignant midline tumors of the posterior fossa in childhood followed by adjuvant therapies like chemotherapy or radiotherapy often leads to longterm survival and even healing of such patients. Therefore, quality of life becomes particular important. Postoperative neurological deficits, such as cerebellar mutism and ataxia have been attributed to splitting of the cerebellar vermis to remove these tumors.

Objective: Here, we tested the effect of vermian splitting in juvenile rats on social behavior, vocalization and motor activity.

Methods: Juvenile male Sprague Dawley rats, aged 23 days, underwent vermian splitting under general anaesthesia after medial suboccipital craniotomy (lesioned group, $n=16$ ). In sham-lesioned rats, only craniotomy was performed and the dura was opened with release of cerebrospinal fluid $(\mathrm{n}=16)$. Naïve rats served as controls $(n=14)$. All groups were tested on day 0 (before surgery), and on days $1,2,3,4$ and 7 after surgery for locomotor activity, motor coordination, social behavior, and ultrasound vocalization during social interaction. Finally, splitting of the vermis was histologically verified.

Results: Social interaction was reduced for two days after surgery in lesioned rats compared to sham-lesioned rats and controls. Vocalization was decreased for one day compared to controls. Locomotor activity was disturbed for several days after surgery in both lesioned and shamlesioned rats as compared to controls.

Conclusion: Deficient social behavior and vocalization after surgery are related to vermian splitting in juvenile rats. These results indicate that similar to the human context vermian splitting can reduce communicative drive in the early postsurgical phase.

\section{Functional and Others}

\section{9}

Restoration of hearing in prelingual deaf children with auditory brainstem implantation

Robert Behr ${ }^{1}$, Friederike Gamm ${ }^{1}$, Jetinder Hans ${ }^{2}$, Mohan Kameshwaran ${ }^{3}$ ${ }^{1}$ Department of Neurosurgery, Klinikum Fulda, Fulda, Germany; ${ }^{2}$ Kapur Memorial Hospital, Delhi, India; ${ }^{3}$ MERF Institute, Chennai, India

Children born with severe cochlear malformation, cochlear aplasia or hypoplasia/aplasia of the cochlear nerve are no good candidates for cochlear implantation (CI). The only possibility for restoration of hearing 
is the implantation of an auditory brainstem implant (ABI). On the basis of a personal 16 years experience in $\mathrm{ABI}$ for neurofibromatosis patients, ABI in children started in 2009. Meanwhile 19 implantations in 18 children, 8 male and 10 female were performed. One child had a successful revision surgery after a fall on the implant side and breakdown of the system. The mean age was 3.5 years, median 3 . The youngest was 1.25 , the oldest $6.5 \mathrm{yrs}$. In 13 aplasia of the cochlear nerve was diagnosed. The others had cochlear dys-aplasia together with hypoplasia of the 8th nerve or syndromal lesions like Goldenhar Syndrome. Surgery was performed in supine position using a retrosigmoid approach and multimodal neuromonitoring. In all cases intraoperative E-BERA were recorded. There were no major complications. All children fitted so far regained sound awareness and insisted using the implant all day. 3 of them already regained word recognition and Ling sound detection. Two started to use speech and one is even bilingual. In conclusion, $\mathrm{ABI}$ is a safe and successful surgical procedure for restoration of hearing and speech in prelingual deaf children. Precious time of plasticity of the auditory pathways should be used as early and intensively as possible. Therefore in doubtful CI candidates ABI should be the primary indication.

\section{0}

Long-term outcome after selective dorsal rhizotomy in children with spastic cerebral palsy

Paul Steinbok ${ }^{1}$, Tamir Ailon ${ }^{1}$, John M. Kerr ${ }^{2}$

${ }^{1}$ Division of Neurosurgery, Department of Surgery, University of British Columbia and BC Children's Hospital; ${ }^{2}$ Faculty of Medicine, University of British Columbia

Background: Most studies of selective dorsal rhizotomy (SDR) demonstrate decreased spasticity, increased range of movement (ROM) and improved motor function. However, there is minimal data on long-term outcomes of SDR. The goal of this study was to evaluate outcomes $>10$ years after SDR.

Methods: Prospective assessments of 44 patients $>10$ years after SDR and comparison with prior assessments done pre-operatively and between 1 and 5 years post-operatively were conducted. Assessments included Ashworth scale for spasticity, ROM, quadriceps strength, motor function and Gross Motor Function Classification Scale (GMFCS) level. Patients were grouped according to pre-operative GMFCS level: Group 1: GMFCS II-III and Group 2: GMFCS IV-V

Results: In both Groups 1 and 2, improved spasticity at early postoperative assessment was maintained after 10 years, but early improved ROM was not maintained after 10 years. Quadriceps strength improved in both groups but took longer in Group 2. Overall motor function improved in Group 1 early but this was not maintained at $>10$ years. In Group 2, no significant improvement in motor function occurred at any time point. For Group 1, compared to preoperatively, 3 of 22 patients had improved GMFCS level at $>10$ years and 4 were worse. For Group 2, 5 of 22 improved GMFCS level at $>10$ years and 1 was worse.

Conclusions: The results indicate that some, but not all, the early improvements after SDR, are maintained in the longer term. This adds information that further delineates the role of SDR in the management of spastic cerebral palsy.

\section{1}

Pediatric neurosurgeons 'risk of facing malpractice claim - The ethical dilemma in pediatric neurosurgeons

Ahmed Ammar

Department of Neurosurgery, King Fahd university Hospital, Dammam University Al Khobar, Saudi Arabia

The medico- legal issues are complex. The recent studies showed the neurosurgeons in the USA, Saudi Arabia and some other countries are among the top 3 subspecialties which are frequently had to face lawsuit claims. The direct and indirect coast of litigation in some cases is devastating In my experience as officially and frequently consulted by the court for expert opinion in the last 20 years, I witness the regular increase of lawsuit claims annually. I was consulted for 28 cases; 15 spine cases, 9 pediatric cases, 3 adult brain tumors, 2 stroke cases and one case of severe head trauma. Summary of some cases will be presented to draw listens from such cases.

Conclusions: Malpractice claims were not related to the medical complexity of a particular case. Pediatric neurosurgery cases comes second after spinal surgery cases constitute the majority of litigations Good communication with the patient and his parents (relatives) is the best insurance against the lawsuit of malpractice Informed consent sometimes is mandatory to protect the neurosurgeon.

152

Delivering bad news in pediatric neurosurgery

Michel Zerah ${ }^{1,}$ Federico Di Rocco ${ }^{1}$, Lesile Pamphile ${ }^{1}$, Aurélia Diaz ${ }^{1}$, Antonio Vecchione ${ }^{2}$, Thomas Baugnon ${ }^{2,3}$

${ }^{1}$ Pediatric Neurosurgery, Necker Hospital, Paris, France; ${ }^{2}$ Anesthesia and Intensive Care, Necker Hospital, Paris; ${ }^{3}$ Simulation Center iLUMENS, Paris Descartes University, Paris, France

Introduction: Teaching the relationship patient-physician is often not considered as important in surgical training, which focuses on surgical skills. However, especially in the pediatric field, parents often complain about the way information has been given to them and their children. These communication difficulties are also increased in case of complex surgery as brain surgery in a child. However, no communication training is provided to most of the pediatric surgical residents. The aim of this pilot study was to evaluate the feasibility for a role-playing based training in announcing difficult news in pediatric neurosurgery.

Materials and Methods: Residents in pediatric neurosurgery were submitted to a role playing scenario in which they had to announce a surgical complication to parents. The session was supervised by a senior neurosurgeon, a psychologist, and a non caregiver observatory. The session was video recorded. The management of the interview, the organization of the announce, the quality of the verbal and non verbal communication were analysed using a pre-established check list of items adapted from SPIKES Protocol. Two actors played the standardized parents.

Results: This pilot study showed that it is possible to simulate a neurosurgical interview and that the communication skills of the neurosurgical residents are extremely variable. All of them displayed some degree of verbal and/or non verbal communication errors.

Conclusion: There is a need to develop such programs of role-playing teaching for surgical resident curricula, especially in pediatric surgical disciplines where the resident has to deal also with the parents and not only with the patient.

\section{3}

Of course parents are anxious during their child's neurosurgery the question is what can we do about it? A prospective qualitative and quantitative study

Ash Singhal, A. Ross Hengel

British Columbia Children's Hospital, Division of Pediatric Neurosurgery, Vancouver, Canada

Introduction: Pediatric Neurosurgery is associated with significant anxiety for caregivers. While preoperative and postoperative anxiety is well studied, there is little literature on anxiety during the procedure itself. If identified, common themes may provide insights that assist the Neurosurgical team in allaying anxiety during procedures.

Methods: We interviewed caregivers of pediatric patients undergoing neurosurgery at our institution. Procedures were done by the first author, and were generally epilepsy surgery patients. Interviews were conducted 
mid-way through scheduled operations, with a semi-structured, openended format to assess qualitative information about goals, concerns, fears and expectations of caregivers waiting for a child during surgery. The State-Trait Anxiety Inventory (STAI) Questionnaire was used to quantitatively assess parental anxiety level.

Results: Caregivers of 10 children were formally interviewed. Situational anxiety was markedly elevated $(\mathrm{p}<0.05)$ in comparison to underlying anxiety. Common qualitative themes emerged: parents cited the importance of surgeon self-confidence in reducing anxiety. The neurosurgery epilepsy nurse was often mentioned as a valuable resource. Interviewees repeatedly stated that providing more realistic surgical time-frames would ease anxiety while waiting. In situations where an accurate surgical timeframe could not be maintained, parents indicated that periodic updates regarding their child's status would be highly beneficial for coping with anxiety.

Conclusion: Caregivers have high anxiety levels during procedures. Our results suggest that by providing families with status updates, realistic surgical time-frames, and access to preoperative specialty nurses, institutions can potentially reduce anxiety experienced by caregivers in the waitroom.

\section{4}

Intra-operative complications in pediatric neurosurgery: review of 1807 procedures

Sebastian Arts ${ }^{1}$, Hans Delye ${ }^{1}$, Laura Blok ${ }^{2}$, Petros Diagos ${ }^{2}$, Erik J. van Lindert $^{1}$

${ }^{1}$ Department of Neurosurgery, ${ }^{2}$ Department of Anesthesiology, Radboud University Nijmegen, Medical Centre, Nijmegen, The Netherlands

Introduction: This study aims to establish the intra-operative complication rate (ICR) of pediatric neurosurgical procedures to the purpose of informed consent of parents, and to establish a baseline for further targeted improvement of pediatric neurosurgical care.

Methods: Analysis of a prospective complication registration database that contains a consecutive cohort of all pediatric neurosurgical procedures in a general neurosurgical department from the start of data collection in January 2004 until June 2012. In the study period 1807 procedures were performed on patients below the age of 17 .

Results: A total of 65 intra-operative complications occurred in 62 patients $(5,4 \%)$. Intra-operative mortality was $0,17 \%(n=3)$. Complications that were related to the neurosurgical procedure itself contained $75 \%$ $(\mathrm{n}=49)$ of complications, while $25 \%(\mathrm{n}=16)$ had an anesthesiological cause. The highest ICR occurred during cerebrovascular surgery $(7,7 \%)$ and tumor surgery $(7,4 \%)$. The most frequently occurring complications were cerebrovascular complications $(28 \%)$.

Conclusion: Intra-operative complications are not uncommon during pediatric neurosurgical procedures. Awareness of these complications is the first step in preventing them.

\section{5}

Surgical results in obstetric brachial plexus lesions (OBPL)

Friederike M. T. Gamm, Robert Behr

Klinik für Neurochirurgie, Klinikum Fulda, Fulda

Introduction: The incidence of OBPL is about 0.5-2.5\%. Spontaneous functional recovery varies from $7-80 \%$. In cases of failed improvement microsurgical intervention is a serious treatment option.

Methods: 70 children with OBPL were included in this restrospective study. All children were clinically examined after birth and thereafter on a regular basis using the Gilbert and Raimondi (G\&R) scores. 48 children improved consistently and showed good recovery of motor function after 3 month and ongoing recovery thereafter. Due to failure of recovery 23 children were operated 6-9 month after birth.
Results: Using the G\&R scores the operated children with upper OBPL (C5,C6 roots, $\mathrm{n}=9$ ) improved from 0.28 to 3.87 (shoulder), from 2.33 to 4.47 (elbow) and form 3.85 to 4.28 (hand). For combined OBPL (C5 $\mathrm{D} 1$ roots, $\mathrm{n}=13$ ) the scores improved from 0 to 2.42 (shoulder), from 1.25 to 3.49 (elbow) and form 0.56 to 2.16 (hand). One child with lower OBPL improved from 0 to 4 (shoulder), from 2 to 4 (elbow) and from 2 to 3 (hand). There were no major complications like hematoma or infection. Conclusion: Out of 71 children 23 were operated after careful examination and failed spontaneous recovery (32.4\%). The recovery of motor function after surgery showed significant improvement. Especially the upper OBPL showed good results but also hand function showed good improvement in combined and lower OBPL. In summary microsurgical reconstruction of OBPL is a safe and effective treatment in carefully selected children.

156

Routine preoperative blood tests in pediatric neurosurgery - are they cost effective?

Fajer Almesbah, Tafadzwa Mandiwanza, Chandrasekaran Kaliaperumal, John Caird, Darach Crimmins

Department of Neurosurgery, Children's University Hospital, Dublin, Ireland

Introduction: The frequency of routine preoperative blood tests in predicting perioperative or postoperative complications is insignificant. The unnecessary ordering of routine tests increases the financial costs and patient's distress. We decided to carry out an audit to evaluate the impact of these tests on patient management and the overall financial costs.

Methods: We retrospectively reviewed the medical records and laboratory data of 355 patients electively admitted to the neurosurgical department over a 5-year period from January 2008 to December 2012. We excluded all patients who were admitted for imaging or surgical procedures requiring local anesthesia. We recorded the results of preoperative and previous (up to 6 months prior to surgery) blood tests and kept note of any abnormalities.

Results: A total of 3489 blood tests were ordered preoperatively in 336 patients (94.6\%), of these $320(9 \%)$ abnormalities were detected. The majority of the abnormalities detected fell close to the reference range and none of the abnormal results had a significant impact on the progression of scheduled procedures. Only one patient $(0.28 \%)$ had her procedure cancelled due to an abnormal preoperative PTT value that turned out to be a false positive after further testing. The overall cost of these tests was 5205 euro to 10,410 euro over the 5-year period.

Conclusions: We concluded that preoperative tests should be selectively requested based on clinical indication.

157

Use of Magnetic Resonance-Guided Laser Thermal Ablation for Children with Intracranial Tumors and Epilepsy: SUNY Upstate Medical University Preliminary Experience

Zulma Tovar-Spinoza ${ }^{1}$, David A Carter ${ }^{1}$, Yaman Eksioglu ${ }^{2}$, Sean Huckins $^{1}$

${ }^{1}$ Department of Neurosurgery, SUNY Upstate Medical University, Syracuse, NY

Laser-induced thermal therapy (LITT) is an emerging technology for the treatment of intracranial pathologies. LITT uses laser energy delivered through a fiberoptic catheter inserted into the lesion through a $3 \mathrm{~mm}$ cranial opening; the ablation is performed under MRI guidance. Its successful use in adult intracranial lesions has increasingly been reported. However, the literature on the use of LITT in pediatric intracranial lesions is limited. Methods: We present the SUNY Upstate Medical University initial experience with the use of LITT in pediatric patients with different 
intracranial tumors/epileptogenic focus. Post-operative and short-term follow-up outcomes are presented.

Results: We treated 8 patients with 10 procedures (4 with intracranial tumors and 4 with epileptogenic focus) between February 2012 - April 2013. The age of the patients ranged from 2 to 18 years. No intraoperative or post-operative complications were reported. Short-term follow-up shows tumor shrinkage/resolution and good seizure outcome. Conclusions: Laser-induced thermal therapy under MRI guidance appears both technically feasible and clinically safe to treat a variety of epileptogenic focuses (hypothalamic hamartomas, cortical dysplasias, cortical malformations, tuberous sclerosis) and difficult intracranial neoplasms. Post-operative and short-term follow-up outcomes are promising. Larger series and long-term outcomes are needed to better define the indications and demonstrate the efficacy of this emerging technology.

\section{8}

\section{Microhemodynamics imaging in peripheral nerves by contact endoscope}

Sebastian Senger, Sonja Vulcu, Karsten Schwerdtfeger, Joachim Oertel Department of Neurosurgery, Saarland University Medical Center and Saarland University Faculty of Medicine, Homburg Saar, Germany

Background: Entrapment neuropathies include a wide field of locations. In many cases, the microsurgical decompression is still the therapy of choice. However, the role of venous stasis and ischemia is still discussed controversially. With a new developed contact endoscope, we demonstrate in vivo visualization of microvessels of peripheral nerves during decompression surgery.

Material and Methods: In five patients suffering from entrapment neuropathies a video assisted analysis of the peripheral nerves was performed. The pathologies ranged from supinator syndrome to neurinomas. After accessing the spot of compression, high definition videos proximal and distal of the compression were recorded. The identical regions were recorded again after decompression of the peripheral nerve.

Results: The use of the contact endoscope caused no intra- or postoperative complications. In the off line computer assisted analysis single arterioles and veins were visualized showing decreased microcirculation before decompression. After treatment an increase in red blood cell flow was observed in all cases.

Conclusion: In all, in this small series, the application of a contact endoscope in peripheral nerve surgery was investigated for the first time. Preliminary results points to a potential role of the microcirculation for the development of symptoms in peripheral nerve entrapment neuropathies. Future studies will show if this endoscope can improve intraoperative minimal invasive diagnosis.

\section{9}

Are preoperative laboratory investigations and cross-match necessary for elective pediatric neurosurgical case management? Michael M. H. Yang ${ }^{1}$, Ash Singhal ${ }^{1}$, Nicholas $\mathrm{Au}^{2}$, A. Ross Hengel ${ }^{1}$ ${ }^{1}$ British Columbia Children's Hospital, Division of Pediatric Neurosurgery; ${ }^{2}$ British Columbia Children's Hospital, Division of Hematopathology

Introduction: Studies in the adult literature suggest preoperative laboratory investigations and cross-match are excessively performed and often do not lead to change in clinical management. The purpose of this study was: 1 . explore whether preoperative laboratory investigations in children alter clinical management, and 2. determine the utilization of cross-matched blood intraoperatively in elective pediatric neurosurgical cases.
Methods: We reviewed pediatric patient charts for elective neurosurgery procedures (2010-2012) at BC Children's Hospital. Variables collected include pre- and postoperative complete blood count $(\mathrm{CBC})$, electrolytes, coagulation, group and screen, and crossmatch. The review also examined for altered clinical management, as a consequence of pre-operative blood work. The number of crossmatched blood units, transfused intraoperatively, was also obtained. Results: 212 patients were reviewed. Pre-operative $\mathrm{CBC}$ was done on 138 and $41 \%$ were abnormal. Coagulation bloodwork was performed in 111 patients and $25 \%$ were abnormal. Out of all the pre-op lab investigations, only 2 patients (both previously on chemotherapy) had lab values in the critical range. Apart from these 2 patients with critical lab values, the bloodwork did not alter clinical management. $65 \%$ of patients had a group and screen and $60 \%$ had blood crossmatched; $6 \%$ of patients $(12 / 212)$ received intraoperative blood transfusion ( $66 \%$ of these patients were under 3 years of age).

Conclusion: This study suggests that the results of pre-operative laboratory exams have limited or no value, apart from cases with oncology and pre-existing conditions. Additionally, there is a need to further refine who receives cross-matching to avoid increased costs and patient discomfort.

\section{Neuro-Oncology II}

\section{0}

Long-term results of congenital brain tumors diagnosed in the first year of life: single institutional experience

Junichi Yoshimura, Masakazu Sano, Hiroshi Aoki, Kenichi Nishiyama, Masafumi Fukuda, Kouichirou Okamoto, Yukihiko Fujii

Department of Neurosurgery, Brain Research Institute, University of Niigata, Niigata, Japan

Introduction: We assessed treatment outcomes and problems associated with congenital brain tumors diagnosed in the first year of life.

Methods: From 1982 to 2012, 21 patients with congenital brain tumors including 4 fetal brain tumors diagnosed in utero were enrolled. Followup periods ranged from 9 months to 29 years (mean, 8.4 years). We retrospectively analyzed their histology, treatments, prognoses and prognostic factors (age, histology, extent of removal, chemoradiotherapy, tumor size, hydrocephalus).

Results: Histopathologically, there were 6 astrocytic tumors, 6 embryonal tumors, 5 choroid plexus tumors (papillomas/carcinomas), 1 anaplastic ependymoma, 1 immature teratoma, 1 anaplastic meningioma, and 1 cavernous angioma. Gross total removal was performed in 7 cases, chemoradiotherapy was administered in 17 , and shunt placement was performed in 10 . The 5 -year overall survival rate was $58.1 \%$. Regarding developmental status, 2 of 10 survivors were normal (papilloma, anaplastic meningioma), 1 showed mild mental retardation (papilloma), 2 moderate mental retardation (astrocytoma, cavernous angioma), and 5 severe mental retardation (hypothalamic astrocytoma, primitive neuroectodermal tumor, immature teratoma, medulloblastoma, malignant astrocytoma). Severe mental retardation was significantly more frequent in malignant tumor cases than in benign tumor cases $(p<0.05)$. In univariate analyses, none of the factors were significantly prognostic, although fetal and newborn cases tended to show better survival $(\mathrm{p}=0.051)$.

Conclusions: Survival of patents with congenital brain tumors was favorable. Favorable functional outcomes were noted in patients with benign tumors, whereas survivors with malignant tumors had extremely poor functional-outcomes. Improvement in their functional prognoses is an important issue. 
161

Intracranial neoplasms in the first year of life: results of a third cohort of patients from a single institution

Greg James, Kim P. Phipps, Mano Shanmuganathan, William F. J. Harkness, Richard D. Hayward, N.u. Owase Jeelani, Dominic N. P. Thompson, Kristian Aquilina

Department of Neurosurgery, Great Ormond Street Hospital for Children, London, UK

Introduction: Brain tumours in first year of life are rare and management is challenging.

Methods: Casenote/database review for patients $<1$ yr with brain tumours since our historical published series of 75 children $^{1}$.

Results: 76 children were identified. Median age at presentation was $180 \mathrm{~d}$ (0-351). M:F ratio was $47: 29.63 \%$ of tumours were supratentorial, $27 \%$ infratentorial and $1 \%$ both. 70 children ( $92 \%$ ) underwent $\geq 1$ neurosurgical procedure, of which $65(85 \%)$ had surgery on the tumour. $25(33 \%)$ had gross total resection, and $25(33 \%)$ required CSF shunt. There were 2 perioperative deaths. The most common tumours were choroid plexus neoplasms $(16 ; 4$ carcinomas $)>$ astrocytomas $(11)>$ PNET (9) $>$ other glioma (7) > ATRT (6) = GBM (6) = ependymoma (6) > teratoma (4), also 11 miscellaneous neoplasms. 34 (45\%) children received chemotherapy and $7(9 \%)$ received radiotherapy. Survival was $91 \%$ at 1 month (68/ $75), 70 \%$ at 6 months (48/69), 64\% at 1 year (44/69), $43 \%$ at 5 years $(24 /$ 56 ) and $27 \%$ at 10 years (12/45). Low-grade gliomas and choroid plexus tumours had the best prognosis, GBM/PNET the worst. At 5 years, $48 \%$ of surviving children were in mainstream education, with $28 \%$ requiring assistance and $24 \%$ in special education. Compared to our previous series ${ }^{1}$, overall survival was unchanged. Choroid plexus tumours were more common, with less PNET (although this reduction was commensurate with number of ATRT in the current series, a diagnosis recognized only recently).

Conclusions: Progress in outcomes, and future direction, in this difficult group will be discussed.

${ }^{1}$ Kane PJ, Phipps KP, Harkness WFJ, Hayward RD (1999) Intracranial neoplasms in the first year of life: results of a second cohort of patient from a single institution. Br J Neurosurg 13:294-8.

\section{2}

\section{Bilateral basal ganglia germ cell tumors: a report of 9 cases}

Hsin-Hung Chen ${ }^{1,6}$, Feng-Chi Chang ${ }^{2,6}$, Yi-Wei Chen ${ }^{5,6}$, Shih-Chieh $\mathrm{Lin}^{4,6}$, Ting-Rong $\mathrm{Hsu}^{3,6}$, Muh-Lii Liang ${ }^{1,6}$, Kai-Ping Chang ${ }^{3,6}$, SangHue Yang ${ }^{5,6}$, Donald M. Ho ${ }^{4,6}$, Tai-Tong Wong ${ }^{1,6}$

${ }^{1}$ Pediatric Neurosurgical Division, The Neurological Institute, ${ }^{2}$ Department of Radiology, ${ }^{3}$ Department of Pediatrics, ${ }^{4}$ Department of Pathology, ${ }^{5}$ Radiotherapy Division, Cancer Center, Taipei Veterans General Hospital and ${ }^{6}$ National Yang Ming University School of Medicine, Taipei, Taiwan, R.O.C.

Objective: Germ cell tumors (GCTs) originating in the basal ganglia (BG) are rare and bilateral cases are even rarer. It is notorious for the diagnostic difficulty and the majority of the patients present symptoms similar to multiple sclerosis or stroke. The authors investigated factors related to the diagnosis of these lesions as well as outcome in order to improve the treatment efficacy.

Materials and methods: We retrospectively reviewed the clinical features, neuroimaging studies, tumor markers, management, and outcome of these 9 patients from 1985 to 2012 in Taipei Veterans General Hospital. Results: 8 of them were boys and 1 was girls. The mean age of onset of symptoms was 11.1 years (range, 8.3-13.6). All but 1 patients presented with at least one side of progressive hemiparesis, 7 with slurred speech, headache, 2 with nausea/vomiting, 3 with diabetes insipidus, and 1 with precocious puberty. 6 patients have histopathological diagnosis: pure germinoma in 4, yolk sac tumor in 2, and mixed GCTs in 1. Bilateral BG GCTs represented delayed diagnosis (usual 1 to 2 years) and poor functional outcome (5 patients were severe disabled or bed-ridden). Postoperative primary adjuvant therapies included radiotherapy, chemotherapy alone, and combined chemotherapy and radiotherapy. All patients survived from 6 months to 18 years (mean follow-up, 61 months). Local recurrence was not observed currently in all of the patients and 1 patients was metachronous bilateral BG GCTs with interval of 5 years.

Conclusion: The BG is a significant locus for intracranial germ cell tumor and can be bilateral. The initial image finding may be subtle and it should be highly suspicious when hemiparesis or precocious puberty presented in children.

\section{3}

Immunohistochemical evaluation for the pathogenesis of intracranial germ cell tumors: expression of pluripotency and cell differentiation markers

Tadashi Miyagawa, Hisayuki Murai, Yasuo Iwadate, Naokatsu Saeki Department of Neurosurgery, Chiba University Graduate School of Medicine, Chiba, Japan

Introduction: Primary intracranial germ cell tumors (iGCT) are rare neoplasms that occur in children and adolescents. This study examined both the pathogenesis and the origin of these tumors, as it has been hypothesized that they originate from a totipotent primordial germ cell. Materials and Methods: We applied recent knowledge from gonadal germ cell tumors and analyzed expression of a wide panel of stem cell-related proteins(C-KIT, OCT-3/4 (POU5F1), NANOG, SOX2, CD30 and PLAP). Expression shown by immunohistochemistry was analyzed in 12 children and young adults with iGCT, contributing to a careful description of these unusual tumors and adding to the understanding of pathogenesis.

Results: Immunohistochemistry showed $5 / 5$ positive in Oct $3 / 4,5 / 5$ in NANOG, $9 / 9$ in C-KIT and 1/5 in SOX2. All cases that showed positive staining in Oct3/4 or NANOG indicated that the tumors contained germinoma or embryonal carcinoma component. Immunohistochemistry revealed that stem cell related proteins were highly expressed in iGCT, and many similarities were detected with their gonadal equivalents, including a close similarity with primordial germ cells.

Conclusion: The expression of genes associated with embryonic stem cell pluripotency in CNS germ cell tumors strongly suggests that these tumors are derived from cells that retain, at least partially, an embryonic stem cell-like phenotype, which is a hallmark of primordial germ cells. In addition, these data can be applied to stratify iGCT for treatment strategy in order to provide good survival rate and good functional outcome.

\section{4}

Epidermoid cyst of the pineal region. An analysis of an experience in seventeen surgically treated cases

Dattatraya Muzumdar, Trimurti Nadkarni, KetanDesai, Atul Goel

Department of Neurosurgery, King Edward VII Memorial hospital and Seth G.S.Medical College, Parel, Mumbai, India

Introduction: Epidermoid tumors are slow growing benign neoplasms. Pineal region epidermoid tumors are rare. Advances in MR imaging techniques have aided in preoperative diagnosis. They have subtle symptomatology, propensity to invade into crevices and tend to be large in size at diagnosis. The long-term outcome of radically resected tumors is excellent. Patient and Methods: Thirty six cases of pineal region epidermoid cysts were surgically treated in our department over the past 16 years (19922010). Seventeen patients were in the pediatric population. A retrospective analysis is presented.

Results: The average age of presentation was 15 years and duration of complaints was 9 months. Headaches and ataxia were commonest complaints. The infratentorial supracerebellar route was used predominantly 
in 13 patients. Total excision of tumor was carried out in 12 patients while near total excision was performed in 3 cases. Dissection around vital neurovascular structures in the vicinity precluded radical excision in the remainder cases. Transient Parinaud's syndrome was seen in three patients but resolved completely at follow-up. One patient who had mild residue developed postoperative meningitis, hydrocephalus, tonsillar herniation and cervicodorsal syringomyelia in the immediate postoperative period. Ventriculoperitoneal shunt was required in one patient postoperatively. There was neither mortality nor any significant morbidity. One patient required reoperation for symptomatic residual tumor. The average follow-up period was 6.3 years. All patients are leading active and independent lives.

Conclusion: Radical surgery for pineal region epidermoid tumors is rewarding and long-term outcome is usually excellent.

\section{5}

Acoustic neurinomas in children and young adults - Analyzing the management issues

Suhas Udayakumaran, Shyam Sunder, A. Parasuraman, Dilip Panikar Division of Pediatric Neurosurgery, Department of Neurosurgery, Amrita Institute of Medical Sciences and Research Centre, Kochi, Kerala, India

Background: Acoustic neurinomas in pediatric age group is a rare lesion. Usually they are associated with neurofibromatosis type 2. Sporadic acoustic neurinomas are even rarer.

Materials and methods: We analysed retrospectively the data of patients with acoustic neurinomas under the age of 25 years between the years January 2001 to March2013. We analysed the age, sex, symptomatology, imaging, surgical strategy, postoperative follow-up and outcome. There were about 28 patients (age range $12-25$, average 21.3 years), 15 males and 13 females. Out of these 10 had definitive features of NF2. Others either had no features or did not show any features even on follow-up. Impaired hearing and headache were the commonest symptoms. 18 surgeries were through retrosigmoid approach, 10 translabyrinthine, 1 transotic, 3 had combined approach. 21 out of 28 (75\%) had documented facial nerve preservation. Average follow-up was 6.7 years (6 lost to follow-up)

Discussion: Acoustic neurinomas in young are a management challenge for few reasons. They are usually larger than the usual occurrence, more aggressive with higher recurrence. Their association with NF2 adds additional challenges. In this study the authors describe their experience of managing these lesions.

Conclusions: We describe our experience of managing acoustic neurinomas of the young

\section{6}

\section{Pediatric skull lesions}

Yasser Jeelani, Stephanie Da Silva, Grace Young, Mark Krieger, J. Gordon McComb

Division of Neurosurgery, Children's Hospital Los Angeles, Department of Neurological Surgery, Keck School of Medicine, University of Southern California, Los Angeles, California

Introduction: Palpable skull lesions encompass a diverse group of histological entities. A review of these masses at one institution was undertaken for further insights.

Methods: An IRB approved retrospective review was undertaken to identify all patients who underwent surgical excision of a skull mass between $1997 \& 2012$.

Results: A total of 279 patients were identified, with no sex predilection (Male:140, F:139); non-malignant lesions totaled 264/279 (95\%) with dermoid cysts 142 (52\%) and Langerhan Cell Histiocytosis (LCH) 31 (11\%) being the most common. Malignant lesions accounted for 15/279
(5\%) and consisted of 4 primary malignancies and 11 metastatic lesions and included a diverse histology. Pain to palpation was noted in 38/279 (14\%), 35 of which were benign with LCH accounting for 17 . Mean age at diagnosis for malignant lesions was not significantly different from those non-malignant, 59 and 69 months, respectively. No significant complication form the surgery was recorded in any patient. At a mean follow-up interval of 17 months, recurrence was encountered in 8/15 malignant lesions and 5/264 benign masses, usually at the original site. Only 42/279 (15\%) had some form of preoperative imaging. Conclusions: The overwhelming majority of skull masses can be completely and safely excised. Preoperative imaging is not needed with obvious lesions such as dermoid cysts.

\section{7}

Interstitial radiosurgery (IRS) with I-125 seeds in children with brain tumors in eloquent areas

Michael Trippel $^{1}$, M. Neuburger ${ }^{2}$, Rudolf Korinthenberg ${ }^{2}$, Volker A. Coenen ${ }^{1}$ ${ }^{1}$ Department of Stereotactic and Functional Neurosurgery, University Hospital, Freiburg, Germany; ${ }^{2}$ Division of Neuropediatrics, Department of Pediatrics and Adolescent Medicine, University Hospital, Freiburg, Germany

Objective: The treatment of brain tumors in eloquent areas is a considerable challenge, especially in children. Since 1990, 318 implantations of Iodine-125 seeds were done in children ( $0-17$, mean $8.83 \pm 4.7$ years). Methods: Retrospectively, patient records and imaging were analyzed. Histopathological diagnoses were pilocytic astrocytoma (174), fibrillary astrocytoma (77), hamartoma (23), oligoastrocytoma (10), anaplastic astrocytoma (7), ependymoma (5) and other lesions (15) located in the suprasellar region (95), thalamus (16), basal ganglia (61), frontal (43), temporal (27), parietal lobe (11), midbrain (26), pineal region (2), pons (9). The most important subgroups low-grade astrocytoma $(79 \%)$, diencephalic (54\%) and brainstem tumors (11\%) were further studied. Results: In the initial Rankin scale $9 \%$ of patients were free of symptoms, $49 \%$ were symptomatic but not disabled, $31 \%$ were slightly, $8 \%$ moderately and $2 \%$ severely disabled. 5 patients had died within a total observation period of $53.6(6.8-171.0)$ months. The time until the indication of a renewed treatment $(\mathrm{n}=47$, of these 18 repeated IRS) or to last follow-up was $38.4(6.4-171.0)$ months. As a result of the first IRS four patients showed complete, 27 partial, 18 objective responses, 15 showed stable and 30 progressive disease. The response did not depend on age, sex, histology, tumor size, location or demarcation of the tumor. Conclusions: Interstitial radiosurgery is a safe, minimally invasive, welltolerated, cost and time efficient neurosurgical procedure with a low rate of morbidity and adverse events, especially in children and adolescents. If no histo-pathological diagnosis is available, verification by a stereotactic biopsy is possible. I-125 IRS is a useful treatment option in the complex treatment of low-grade brain tumors.

\section{8}

Overcoming brain shift during neuronavigation-guided glioma surgery

Masahiro Nonaka ${ }^{1}$, Yohei Bamba ${ }^{1}$, Shin Nakajima ${ }^{1}$, Mami Yamasaki ${ }^{2}$

${ }^{1}$ Department of Neurosurgery, Osaka National Hospital, Osaka, Japan; ${ }^{2}$ Department of Pediatric Neurosurgery, Aijinkai Takatsuki Hospital, Takatsuki, Japan

Recent development of imaging technology enables to visualize information about tumor and neuroanatomy, such as PET and DTIs. Many of these images are possible to integrate to navigation system. However, by dural opening and by surgical resection of the tumor, brain shift occur. This shift makes preoperative image obtained for navigation unreliable. To overcome this problem, we developed an implantable marker probes made from shunt tubes. These markers were embedded into the planned target under navigation guidance immediately after craniotomy, before 
brain starts to shift, and were considered as the landmark. The usefulness of this technique was assessed by evaluating resected tumor volume and perioperative performance status. From 2009, 60 glioma operations were carried out using this method, and 5 patients were pediatric gliomas under 15 years old. Resection rates of Gd-enhanced areas were used for evaluation for tumors with enhanced lesion $(\mathrm{n}=33$, group $\mathrm{A})$ and resection rates of FLAIR-high signal areas were used for nonenhanced lesions $(n=27$, group B). Preoperative and postoperative performance status were measured based on Karnofski performance scale (KPS). Mean resection rate of the group A was $96.6 \%$. Mean preoperative KPS was $67.4 \%$, and postoperative KPS showed improvement upto $76.1 \%$ in group A. Mean resection rate for group B was $84.4 \%$, Mean preoperative KPS was $95.0 \%$ while postoperative KPS was $91.8 \%$. There was no procedure-related complication in both groups. There was no complication due to marker insertion under navigation guidance. Using this method, higher resection rate with improvement of performance status may be achieved.

\section{9}

\section{Tractography and neuronavigation: a new important tool} for optimizing brain lesions resection?

Giselle Coelho $^{1}$, Filipe Kunsler ${ }^{1}$, Rainer Haetinger ${ }^{2}$, Ricardo Mendes Rogério $^{2}$, Sidnei Epelman ${ }^{3}$, Nelci Zanon ${ }^{1}$

${ }^{1}$ Pediatric Neurosurgery Center / CENEPE, Beneficência Portuguesa Hospital, São Paulo, Brazil; ${ }^{2}$ Department of Radiology, Beneficência Portuguesa Hospital, São Paulo, Brazil; ${ }^{3}$ Department of Pediatric Oncology, Santa Marcelina Hospital, São Paulo, Brazil

Introduction: Diffusion tensor imaging (DTI) is a promising, noninvasive magnetic resonance imaging (MRI) method for studying the anatomical organization of major white matter fiber systems. A topic, which currently is receiving increased attention, is the application of DTI fiber tracking in the investigation of patients with intracranial tumors. Therefore, has been used in neuronavigation systems, allowing the correct and precise tumoral identification and, consequently, safe resection.

Methods: Structural magnetic resonance imaging, and diffusion tensor imaging were performed on 12 patients before they underwent lesion resection using neuronavigation. 11 patients presented brain tumors and six of them were localized in posterior fossa. One case, the diagnostic of the supratentorial lesion was brain abscess. The clinical feasibility of this approach was evaluated in 12 cases of lesion resection. In addition, system performance was evaluated by measuring the latency between surgical tool tracking and visualization of the white matter tracts.

Results: Lesion resection was performed successfully in all 12 patients. The seeded white mater tracts close to the lesion and other critical structures, as defined by the structural images, were interactively visualized during the intervention to determine their spatial relationships relative to the lesion and critical deep cortical areas.

Conclusion: Tractography can be used combined with neuronavigation for guiding resection of tumors. Even more important, this serie shows why tumor resection surgery based on tractography mapping may sometimes lead to better clinical outcomes (mainly in low-grade gliomas) even though in posterior fossa, without presenting notable brain shift.

\section{0}

Pinealoblastoma in children: about a series of 9 children treated between 2000 and 2010

Alexandru Szathmari ${ }^{1}$, Blandine Grassiot ${ }^{1}$, Pierre-Aurélien Beuriat ${ }^{1}, \mathrm{C}$. Rousselle $^{1}$, Didier Frappaz ${ }^{2}$, L. Claude ${ }^{2}$, A. Jouvet ${ }^{3,4}$, Carmine Mottolese $^{1}$

${ }^{1}$ Service de Neurochirurgie pédiatrique E, Hôpital Pierre Wertheimer, Groupement hospitalier EST, Hospices civils de Lyon, Bron, France; ${ }^{2}$ Institut d'Hématologie et d'Oncologie Pédiatrique, Centre Léon Bérard, Lyon; ${ }^{3}$ Service de Pathologie et de Neuropathologie, Centre de Biologie et Pathologie Est, Groupement Hospitalier Est, Hospices Civils de Lyon, France; ${ }^{4}$ Centre de Recherche en Neurosciences de Lyon, INSERM U1028,
CNRS UMR5292, Equipe Neuro-Oncologie et Neuro-Inflammation, Lyon, France.

Introduction: Despite progress, pinealoblastoma in children still remains a severe illness. Multidisciplinary approach has not increased the significantly the survival as for other PNET in children. We reviewed the outcome in a series of children treated in our unit in the past decade.

Methods: Between 2000 and 2010, 9 cases of pinealoblastoma were treated in our service. The mean of age of the group was of 44 months. Most frequent clinical presentation was the intracranial hypertension. Four children need surgery for hydrocephalus, 3 before and 1 after surgery. All children were treated by surgery. Treatment was completed by cranio-spinal radiotherapy $(n=4)$ and/or chemotherapy $(n=9)$ according to the extension of the disease.

Results: All but 4 patients died: one at 7 months and 4 after surgery $(8,10$, 16 and 25 months). Four patients are alive, 3 children are going to school, one in normal course and 2 need assistance.

Discussion: The pinealoblastoma remains a still severe illness, more than the medulloblastomas of the posterior fossa. The poor survival seems still better in children than in adults. The surgical removal is difficult because of the regional adherence of the tumour, notably to the Galien ampulla.

\section{Neuro-oncology III}

\section{1}

Residual tumor in pediatric ependymoma: Surgical difficulties and neurological outcome

Kara Krajewski ${ }^{1}$, Katja von Hoff ${ }^{2}$, Gertrud Kammler ${ }^{1}$, Carsten Friedrich ${ }^{2}$, André von Bueren ${ }^{2}$, Rolf-Dieter Kortmann ${ }^{3}$, Jürgen Krauss ${ }^{4}$, Monika Warmuth-Metz ${ }^{5}$, Stefan Rutkowski ${ }^{2}$

${ }^{1}$ Neurosurgical Clinic, University Medical Center Hamburg-Eppendorf, Germany; ${ }^{2}$ Department of Pediatric Hematology and Oncology, University Medical Center Hamburg-Eppendorf, Germany; ${ }^{3}$ Department of Radiation Oncology, University of Leipzig, Germany; ${ }^{4}$ Department of Neurosurgery, University of Wuerzburg, Germany; ${ }^{5}$ Department of Neuroradiology, University of Wuerzburg, Germany

Introduction: Extent of resection is the strongest prognostic factor in intracranial pediatric ependymoma. Postoperative residual tumor was quantified and compared with neurological outcome.

Methods: Analysis of a subcollective of patients with localized ependymoma treated within the multicenter HIT2000 study. Surgical reports were analyzed according to radicality and intraoperative difficulties. Pre- and post-operative neurological statuses were compared.

Results: 221 patients operated in 70 centers were evaluated. 118 (53\%) had complete tumor removal (R0) as determined by central neuroradiological reference, $66(30 \%)$ were $\mathrm{R}+$, and 37 indeterminable (17\%). $\mathrm{R}+$ was found in $19 / 48(40 \%)$ supratentorial hemispheric, $28 / 90(31 \%) 4$ th ventricle/cerebellar, and 15/33 (45\%) tumors involving the cerebellarpontine angle. In 40/66 available surgical reports in the $\mathrm{R}+$ group, gross total resection was described in 11 . Reasons for intentionally leaving tumor were: biopsy(9), localization(6), infiltration of cranial nerves/ arteries(7), bradycardia(2), adherent tumor capsules(2), and other(3). 24/66 patients with $\mathrm{R}+$ underwent reoperation 3 days- 13 months after initial surgery, 15 before / 9 after adjuvant therapy. 10/24 were R0 after reoperation. 3-year EFS for 10 secondary R0 patients was $88 \%( \pm 12)$ vs. $41 \%( \pm 7)$ for $\mathrm{R}+$ patients without second surgery or persisting $\mathrm{R}+$ $(p=0.03)$. Pre/postoperative neurological statuses were compared for altered consciousness, cranial nerve deficits, focal weakness and coordination disturbances; there were no differences among the $\mathrm{R} 0, \mathrm{R}+$ and second look surgery groups.

Conclusions: Neurological outcome is not worse in re-operated patients, whereas EFS is significantly improved in secondary R0 patients. Thus, re-operation should always be considered. 
172

Surgical aspects of pilomyxoid-spectrum astrocytomas in pediatric age group: a prospective cohort study

Mohamed A. El Beltagy ${ }^{1}$, Mostafa M. Atteya ${ }^{2}$, Alaa El-Haddad ${ }^{3}$, Madiha $\mathrm{Awad}^{4}$, Hala Taha ${ }^{5}$, Mohamed Kamal ${ }^{6}$

${ }^{1}$ Chief Neurosurgeon, Children's Cancer Hospital Egypt; Assistant professor of neurosurgery, Kasr Al-Ainy School of Medicine, Cairo University, Egypt; ${ }^{2}$ Assistant specialist of neurosurgery, Children's Cancer Hospital Egypt; ${ }^{3} \mathrm{Head}$ of Pediatric Oncology Department, Children's Cancer Hospital Egypt; ${ }^{4}$ Consultant of pediatric oncology, Children's Cancer Hospital Egypt; ${ }^{5}$ Head of Clinical Pathology Department, Children's Cancer Hospital Egypt; ${ }^{6}$ Research department, Children's Cancer Hospital Egypt

Introduction: Cerebellar pilomyxoid astrocytomas (PMAs) and intermediate pilomyxoid astrocytomas (IPAs) are collectively called "pilomyxoidspectrum astrocytomas (PMSAs)". Cerebellar PMSAs are thought to behave more aggressively than pilocytic astrocytomas (PAs). Our objective is to compare PMSAs to PAs in terms of surgical and clinical profile and outcomes. '

Methods: We evaluated clinical and surgical data of children treated between July 2007 and November 2012 at Children's Cancer Hospital Egypt (CCHE-57357). There were 66 cases with cerebellar astrocytomas (44 PAs, 10 IPAs, and 12 PMAs). Comparison between all groups was performed focusing on brainstem invasion, intrinsic necrotic cavitation, extent of resection, and Recurrence. Statistical analysis was performed using Pearson Chi-Square and One-Way Anova tests (SPSS-V.17).

Results: Cerebellar PMAs and IPAs separately and collectively have higher incidence of brainstem invasion, intrinsic necrotic cavitation and tumor recurrence when compared to PAs (p-value $<0.001)$. Gross total resection was $0 \%$ in PMSAs Vs $84.1 \%$ in PAs (p-value $<0.001$ ). PMAs have higher incidence of tumor recurrence than IPAs $(66.7 \%)$ versus $(20 \%)$, ( $\mathrm{p}$-value $=0.04)$. Incidence of recurrence in PAs was $9.1 \%$ in partially resected cases.

Conclusions: Cerebellar PMA and IPA have significantly lower rates of complete resection when compared to PA. PMAs have higher incidence of recurrence than IPAs. These tumors may represent a clinical spectrum; at its benign end lies PA, while PMA lies at the aggressive end, with IPA lying just behind. These concepts could help guiding management in the future. To the best of our knowledge, this is the largest comparative study for cerebellar pilomyxoid-spectrum astrocytomas.

\section{3}

\section{Management of intracranial teratomas in children: The Taiwan experience}

Tai-Tong Wong ${ }^{1,2}$, Muh-Lii Liang, Hsin-Hung Chen, Kai-Ping Chang, F. K. Chang, Yi-Wei Chen, A.-H. Yen ${ }^{2}$

${ }^{1}$ Pediatric Neurosurgery Department of Surgery, Cheng Hsin General Hospital; ${ }^{2}$ Pediatric Brain Tumor Group, Neurological Institute, Taipei Veterans General Hospital, National Yang Ming University, School of Medicine

Objective: We review intracranial teratomas for demography, managements, and outcomes.

Materials and methods: A total of 57 cases were reviewed. Tumor types, location of tumors, serum tumor markers, disseminations at diagnosis, associated hydrocephalus, surgical resection, adjuvant treatment, and outcome were analysis.

Results: The mean age was 7.9 years. Tumor types include mature teratoma (10 cases), immature teratoma ( 24 cases), and mixed germ cell tumors with teratoma component ( 23 cases). These tumors were mainly located in pineal/pineal sellar region (29 cases), sellar region (10 cases), basal ganglia ( 8 cases), and ventricle ( 9 cases). All of the cases had tumor excision and histological diagnosis. Near total to total resection was performed in 31 patients (54.4\%). Hydrocephalus occurred in 46 cases.
VP shunt, SP, and etV was performed in 25, 2, and 8 patients respectively. Excluded cases of operative mortality, 11 patients completed radiotherapy (RT) treatment, and 23 patients completed radiochemotherapy (RT-CMT) treatment. With followed up period of 7.3 years, 34 patients survived, 18 patients died, and 1 patient loss follow-up from first discharge.

Discussion and conclusion: In general, management of intracranial teratomas follows the classification of prognostic groups. For tumors with $\mathrm{SAFP} \uparrow$ or MGCT component, RT or RT-CMT will benefit for better survival. For tumor with normal sAFP and no MGCT component, individualized optimal treatment should be considered to avoid over treatment and treatment toxicity. IMT in infant of $<1 \mathrm{yr} / \mathrm{o}$ is different from IMT in older child. The management is mainly radical resection alone and second look surgery is important for the necessity of salvage adjuvant treatment.

\section{4}

Is there a dorsally exophytic growth pattern in pilocytic brainstem astrocytomas?

Christian Strauss, Christian Scheller

Department of Neurosurgery, Martin-Luther University of HalleWittenberg, Germany

Introduction: The growth pattern of pilocytic brainstem astrocytomas remains challenging in these rare lesions, but its understanding in the individual patient is essential for a tailored approach, complete removal and quality of life.

Methods: From a series of 500 patients operated upon for posterior fossa lesions between 2002 and 2013, 21 pediatric and young adult patients were identified with pilocytic astrocytomas and evaluated with respect to growth pattern, extent of resection on MRI and functional outcome.

Results: A dorsally exophytic growth pattern could not be identified in any of the cases. Three each harbored focal endophytic and cerebellar tumors. 15 peduncular lesions (midline approach $n=9$, retrosigmoid $n=4$, combined $n=2$ ) were identified and further investigated. Out of these 15 cases a growth pattern from the peduncle into the $4^{\text {th }}$ ventricle, mimicking a dorsally exophytic growth was seen in 9, a lateral growth towards the CPA in 4 cases. In two cases a lateral to medial growth pattern from the peduncle was encountered, pushing the floor of the ventricle dorsally. Tumor removal was complete in all cases but two. Additional morbidity was seen with the midline and combined approach.

Conclusions: The understanding of growth patterns in peduncular astrocytomas provides a safe surgical corridor and is essential for complete removal and functional integrity. The entity of a dorsally exophytic pattern needs to be discussed, since all cases, appearing as dorsally exophytic on MRI, eventually had a peduncular origin with respect to the vascular supply, predominantly coming from the peduncles and not from the ventricular floor.

\section{5}

\section{Long-term results of the combined therapy in patients} with medulloblastoma

Serge Gorelyshev, O. Medvedeva, N. Mazerkina, E. Khukhlaeva, O. Krotkova, M. Ryzhova

Burdenko Institute of Neurosurgery, Moscow, Russia

In 2005-2006 58 children with medulloblastoma were operated at the age 3-18. Postoperative mortality rate was $0 \%$. All patients were treated by protocols CCG and HIT-2000. The overall 5-year survival was 75\% (5RFS - 72\%.) Radical resection was performed in $66 \%$ of cases, subtotal in $34 \%$. 5-RFS for the radical removal of the tumor was $76 \%$ (subtotal$55 \%$ ). Endocrine disorders were detected in all patients - GH deficiency (89\%), hypothyreosis (58\%), hypogonadism (boys $-71 \%$, girls - 79\%), hypocorticism $(12 \%)$. Endocrine deficit was more severe in the patients treated by HIT-2000 $(\mathrm{p}<0,05)$. Neuropsychological disorders were 
detected in all patients - deterioration of praxis $(90 \%)$, visual gnosis $(60 \%)$, constructive activity $(58 \%)$, speech $(50 \%)$, memory (50\%), mental activity (46\%), attention (45\%), general activity (30\%). It was shown that the younger the child at the beginning of combined treatment, the greater the degree of neuropsychological disorders. The critical age was defined at 6 years old (Mann-Whitney U-Test). The influence of molecular genetic pattern was also statistically significant: 5y-RFS rate for WNT group was $100 \%$, D $-80 \%$, SSH $-72 \%$ and $\mathrm{C}-45 \%$.

Conclusions: A complex of neurological, endocrine and neuropsychological disorders manifestate in the follow-up period after the combined treatment of patients with medulloblastoma. Risk factors are a standard craniospinal irradiation and long-term chemotherapy. Adequate stratification, use of protocols considering molecular genetic pecularities of a tumor, providing the reduced dose of craniospinal irradiation in standard risk group would reduce the number of complications in the follow-up period.

\section{6} Advantages, drawbacks, and controversy of alpha-Interferon
intracystic treatment for craniopharyngiomas

Paolo Frassanito, Massimo Caldarelli, Luca Massimi, Gianpiero Tamburrini, Concezio Di Rocco

Pediatric Neurosurgery, Catholic University Medical School, Rome, Italy

Introduction: Clinical trials demonstrate the effectiveness of intracystic alpha-Interferon (IFN) in the control of craniopharyngiomas (CRF). Its drawbacks and impact on long-term management of CRF have not been addressed yet.

Methods: We retrospectively reviewed 8 consecutive cases of CRF, treated with intracystic IFN in our Institute. Clinical and radiological responses were assessed, with particular attention on the actual effectiveness of the drug (tumor regression/growth control), the advantages of the therapy (persistent absence of secondary visual and endocrine deficits), and its impact on the subsequent management of CRF.

Results: Failure was observed in 2 children, whereas cyst regression and stable solid component were initially obtained in 6 subjects. Among them we observed 2 relapses, one case requiring an immediate surgical excision. At surgery, the capsula of CRF was found to be more fibrotic and adherent to the surrounding vascular and neural structures than in usual cases. Although these cases had a similar cyst reduction following treatment, they differ for the MRI characteristics at the tumor-hypothalamus interface.

Conclusions: Intracystic IFN may temporarily control CFR, thereby allowing in young children the maturation of hypothalamus-pituitary axis. Its effect on the cystic component preserves the visual function. The possible occurrence of abrupt and rapid progression of the tumor during treatment suggests very cautious attitude when conservative policy is adopted, especially if IFN made CRF amenable to surgical excision. As in case of relapse the surgical removal may be particularly difficult, a "prophylaptic" excision of CRF could be considered even in cases who have responded favorably to IFN.

\section{7}

The effect of chemotherapy on optic pathway gliomas and their sub-components - a volumetric MR analysis Ben Shofty ${ }^{1,2}$, Michal Mauda-Havakuk ${ }^{2}$, Dafna Ben-Bashat ${ }^{2}$, Rina Dvir $^{2}$, Li-Tal Pratt ${ }^{2}$, Lior Weizman ${ }^{2}$, Leo Joskowicz ${ }^{2}$, Michal Tal ${ }^{2}$, Lior Ravid $^{2}$, Liat Ben-Sira ${ }^{2}$, Shlomi Constantini ${ }^{1,2}$

${ }^{1}$ Department of Pediatric Neurosurgery, ${ }^{2}$ The Gilbert Israeli NF Center, Dana Children's Hospital, Tel-Aviv Medical Center

Optic pathway gliomas (OPG) represent $5 \%$ of pediatric brain tumors. Introduction of novel volumetric imaging methods enable accurate assessment of the tumoral changes under chemotherapy. In this study we examined the effect of fist line chemotherapy treatment with vincristine and carboplatin on the gross total solid volume (GTSV) of the tumor as well as its various sub-components. The tumors of 12 patients ( 7 boys, 7 $\mathrm{NF} 1$, mean age at treatment initiation was 7 years $( \pm 6 \mathrm{y}))$ with OPG were measured using our novel, previously described method. Two MR studies per patient were included in this study. One MRI performed before initiation of treatment, and one after a period of at least 6 months on the protocol. Volumetrics of the gross total solid volume, solid-nonenhancing, solid-enhancing, and cystic components were calculated. The relative percentile change of each of the component following treatment was calculated. During the treatment period with vincristine and carboplatin an average reduction of $8 \%( \pm 27 \%)$ in the GTSV was noted. Solid-non-enhancing components were reduced by an average of $12 \%( \pm 41 \%)$, solid-enhancing were reduced by $17 \%( \pm 78 \%)$ and the cystic component grew under therapy by an average of $25 \%( \pm 86 \%)$. Two patients had de-novo appearance of a cystic component. Treatment with vincristine and carboplatin seems to have an effect mostly on the solid components. The cystic component seems to be unaffected by chemotherapy and contributes to the subsequent growth of the gross total volume, but not of the GTSV.

*This study was supported by a grant from the Israeli Cancer Research Foundation (ICRF) to SC and BS, and by the Gilbert Israeli Neurofibromatosis Center (GINFC).

\section{8}

O-(2- $\left[{ }^{18}\right.$ F]fluorethyl)-L-tyrosine PET in the predictive clinical evaluation of primary pediatric brain tumors

Sevgi Sarikaya-Seiwert ${ }^{1}$, Thomas Beez ${ }^{1}$, Gabriele Stoffels ${ }^{3}$, Gisela Janßen ${ }^{2}$, Hans-Jakob Steiger ${ }^{1}$, Daniel Hänggi ${ }^{1}$

${ }^{1}$ Department of Neurosurgery, ${ }^{2}$ Department of Pediatric Oncology, -Hematology and Clinical Immunology, Heinrich-Heine-University, Düsseldorf, Germany; ${ }^{3}$ Institute of Neuroscience and Medicine (INM$3,-4,-5)$, Forschungszentrum Jülich, Jülich, Germany

Objective: The aim of the study was to determine the predictive value of $\mathrm{O}-\left(2-\left[{ }^{18} \mathrm{~F}\right]\right.$ fluorethyl)-L-tyrosine (FET) in suspected primary childhood brain tumors and the clinical value of dynamic FET-PET for non-invasive tumor grading in this group of patients.

Methods: 32 FET-PET scans were performed in 22 patients (mean age, 13 $\pm 3 \mathrm{y}$ ) with newly diagnosed cerebral lesions suggestive of neoplastic tissue $(n=15)$ or to rule out tumor progression $(n=7)$. Maximum and mean tumor/brain ratios (TBRmax, TBRmean) of FET uptake were determined. Time-activity curves (TAC) were generated and the time-topeak (TTP) was calculated. TACs of each lesion were assigned to one of the following curve patterns: (I) constantly increasing FET uptake; FET uptake peaking early (TTP $\leq 25 \mathrm{~min}$ ) followed by a (II) plateau or (III) a constant descent. Lesion/brain ratios of FET uptake $\geq 1.6$ and a kinetic pattern II/III in combination of a TTP $\leq 25$ min were considered as reference to tumor tissue. The results of the FET-PET studies were confirmed histologically.

Results: In 15 patients with newly diagnosed cerebral lesions suggestive of neoplastic tissue/glioma on MRI, FET PET identified 11/12 lesions correctly as tumors and 2/3 lesions as non-neoplastic (accuracy, 87\%). During the course of the disease, serial FET PET imaging findings of 7 patients correctly identified presence $(5 / 6)$ or absence $(11 / 11)$ of tumor progression/residual tumor (accuracy, 94\%).

Conclusions: These results indicate that FET-PET can add relevant additional information for treatment planning and monitoring in children with brain tumors when MRI is insufficient.

\section{9}

Bithalamic tumors in children

G. Paternoster ${ }^{1}$, Stephanie Puget ${ }^{1}$, Pascale Varlet ${ }^{2}$, C. Dufour ${ }^{3}$, Thomas Blauwblomme ${ }^{1}$, Michel Zerah ${ }^{1}$, Christian Sainte-Rose ${ }^{1}$ 
${ }^{1}$ Pediatric Neurosurgical Unit, Necker Hospital, Paris Cité Sorbonne University, Paris, France, ${ }^{2}$ Neuropathological Unit, Sainte-Anne Hospital, Paris Cité Sorbonne University, Paris, France; ${ }^{3}$ Pediatric Oncology Unit, Gustave-Roussy Institute, Villejuif, France

Introduction: Pediatric bithalamic tumors are rare with only few cases described in literature. The natural history, the histopathology and the best therapeutic strategy are not yet well established.

Methods: We retrospectively reviewed 19 patients $(12 \mathrm{M} / 7 \mathrm{~F})$ admitted on between 1986 and 2012 at Necker Hospital with a median age of 7,5 years (range 3-12 years). We analysed clinical status, radiological features, surgical procedures and adjuvant treatment results, as well as literature data.

Results: We observed signs of intracranial hypertension in $60 \%$ of children, with motor deficit in $25 \%$ and behavioural troubles/epilepsy in less than $10 \%$. Sixty two percent of cases revealed a radiological diffusion of the disease in the contiguous areas, $31 \%$ had contrastenhancement and $62,5 \%$ presented with hydrocephalus. Overall, we realized 26 surgical procedures (16 biopsies and 10 incomplete resections). The histological analysis showed 10 low-grade and 9 high-grade gliomas. All patients received chemotherapy and 11 were also treated by radiotherapy, at diagnosis or at the time of progression. With a follow-up of 7 months to 23 years, we observed a mortality rate of $70 \%$. This result is consistent with literature, however we observed long survivors (up to 23 years).

Conclusion: The results of this series reflect the heterogeneity of these lesions that would benefit from molecular analyses as the more aggressive cases have similar genomic profiles to those of Diffuse Intrinsic Pontine Gliomas.

\section{0}

Recurrence factors in craniopharyngiomas: Study of clinicomorphologic and histopathologic factors

Ashok Kumar Mahapatra, Deepak Kumar Gupta, Anand Gupta, Chitra Sarkar, Ashish Suri, Vaishali Suri, S. S. Kale, B. S. Sharma

Department of Neurosurgery, All India Institute of Medical Sciences, New Delhi, India

Background: Controversity persists in extent of resection in these benign but challenging tumors.

Objective: We performed a retrospective analysis of all craniopharyngiomas operated at our hospital between $1965-2010$ to determine changing practice of management of these tumors over last 5 decades.

Methodology and Observations: 860 cases of histopathologically proven craniopharyngiomas [ 405 children ( $<18$ yrs) / 455 adults, 537 males / 323 females)]. Radical/total excision in 179 cases [(perioperative mortality $(25 / 179,14 \%)$, postoperative DI $74.5 \%]$. Subtotal excision in 577 cases (67\%), perioperative mortality $6.3 \%$, postoperative DI $34.7 \%)$ ]. Recurrence noted in $83 / 497(16.7 \%)$ patients ( $>6$ mo-5 yr follow-up). Adamantinous pattern noted in 437/506 (86.4\%) cases while papillary tumors noted in $47 / 506$ cases. Perioperative mortality was noted in 63 / 860 cases $(7.3 \%)$. Brain invasion noted in $82 / 437$ (18.7\%) cases of adamantinous tumors (pediatric adamantinous tumors had significantly more brain invasion than adult counterparts). No papillary tumor showed brain invasion. Extent of resection influenced recurrence pattern more than histopathology or radiation therapy postoperatively in subtotal excision group.

Conclusions: There is a shift from subtotal excision (1965-1980s) to gross total tumor excisions (1980-2002) and again to subtotal tumor excisions. High perioperative mortality, high incidence of diabetes insipidus in total excision group and less benefit of postoperative radiotherapy s noted in present series. Subtotal excision group outcome compared favourably with total excision group in long-term follow-up.

\section{Poster Presentations}

\section{Brain Malformation}

\section{1}

Chiari 1 malformation in adult patients: common presentations, treatment modalities, and outcome

Nazik Farouk Hussein Ahmed

National Neuroscience Institute, Neurosurgery Department, King Fahad Medical City, Riyadh, Saudi Arabia

Objective: The objective of this study is to describe the presentations, treatment modalities, and outcomes of Chiari 1 malformation.

Methods: This is a retrospective study based on chart review of Chiari 1 malformation in adults (defined in Saudi Arabia as above 12).

Results: We reviewed 11 charts of patients operated on in our institute in the time period between $2005 \& 2012$. The age ranged from 16 to 60 year with mean 30 years. The males were 5 and females were 6 . The common presenting symptoms were headache \& neck pain 9 (81\%), impaired sensation in the left or right half of the body $6(54 \%)$, sleep apnea 3 $(27 \%)$, unsteady gait $2(18 \%)$, hemiparesis $2(18 \%)$, and decreased vision $1(9 \%)$. Neurological examination showed no deficits $3(27 \%)$, hemiparesis $3(27 \%)$, hypothesia in the left or right half of the body 3 (27\%), cerebellar signs $2(18 \%)$, facial palsy $1(9 \%)$, and hypoglossal and partial vagus nerve palsy $1(9 \%)$ patient. MRI study showed cerebellar tonsillar herniation in all patients. Seven (63\%) patients had associated syringomyelia, $4(36 \%)$ had no syringomyelia, $1(9 \%)$ had scoliosis, and $1(9 \%)$ had an odontoid peg and a short clivus, and $1(9 \%)$ had chordoma. All 11 patients underwent suboccipital craniectomy with $\mathrm{C} 1$ laminectomy $\&$ duraplasty. Two (18\%) had additional partial C2 laminectomy and 1 $(9 \%)$ had tonsil dissection and removal of the foramen magnum fibrous band and $1(9 \%)$ had ventriculo-peritoneal shunt insertion. Several complications were found. Pseudomeningiocele $2(18 \%)$, bleeding $1(9 \%)$, and sub-dural hygroma $1(9 \%)$.

Significance: This study has shown that Chiari 1 malformation has a good outcome overall.

\section{2}

Endoscopic management of suprasellar arachnoid cysts

Chidambaram Balasubramaniam

Kanchi Kamakoti CHILDS Trust Hospital, Chennai, India

Introduction: Suprasellar Aracnoid Cysts (SSAC) are an uncommon variety of arachnoid cysts manifesting in many ways in childhood including symptoms of raised intracranial pressure and Bobble Head Doll Syndrome.

Methods: Our experience in the management of five (5) cases of SSAC is presented. Data were analysed retrospectively.

Results: The ages ranged from $1-10$ yrs. The commonest manifestation was increased intracranial pressure. One presented with endocrine deficit (short height) in addition to raised ICP. None had the classical Bobble Head Doll Syndrome. One child presented acutely due to raised ICP. All underwent endoscopic surgery. The five patients underwent eight operations. Five primary endoscopic (cystoventriculostomy and cystocisternostomy) sugeries were done, in one due to bleeding and poor identification the procedure could not be completed. One needed a second endoscopic surgery and two had a shunt placed. The cyst was fenestrated into the right lateral ventricle and then through this fenestration the floor of the cyst/III ventricle was fenestrated into the prepontine cistern. There were side effects in two patients. One developed a contralateral III nerve palsy and the other developed "recurrence" due to closure of the fenestrations needing endoscopic refenestration. VP shunt was done later to 
manage CSF leak. The technique will be described in detail. The longterm results have been good.

Conclusions: The ideal therapy for SSAC is endoscopic cyst fenestration into the ventricle and the prepontine cistern. Cystoventriculostomy alone does not give the best results. Endoscopic cystoventriculostomy and cystocisternostomy give better results.

Spontaneous resolution: an end of line for suprasellar arachnoid cysts

Patricia Barrio, D. Santamarta, A. Mostaza, J. Pérez

Department of Neurosurgery. University Hospital of León, León, Spain

Introduction: Most arachnoid cysts are quiescent and remain asymptomatic throughout life. They can also progressively enlarge and, less frequently, disappear spontaneously.

Methods: We report a case of spontaneous resolution of a suprasellar arachnoid cyst in a child. The cyst was diagnosed incidentally during a transfontanellar ultrasonography study. MRI performed when the child was 11 months old revealed a suprasellar arachnoid cyst, which extended into the middle cranial fossa bilaterally and compressed the midbrain and the third ventricle. No neurological symptoms were detected at that time, pituitary function was normal and surveillance was adopted. The child has remained asymptomatic throughout his early few years. The size of the cyst has decreased noteworthy, being only evident in the right temporal fossa, as it is shown in a MRI performed at the age of seven years. Parents denied any trigger event as head trauma or Central Nervous System infection. The relevant literature was reviewed giving particular interest to possible mechanisms underlying the spontaneous resolution of arachnoid cysts.

Results: Since 1985 nineteen cases of spontaneous resolution have been reported, three of them in suprasellar location.

Conclusions: The lifelong clinical behaviour of arachnoid cysts cannot be reliably ventured. Spontaneous resolution may be more common than it is suggested in the literature. This unexpected and favourable outcome focuses the pathophysiology of arachnoid cysts on an altered pattern of CSF dynamics within the cyst due to hindered communication between the cyst content and the subarachnoid space. A conservative management is the most rationale approach for asymptomatic cases.

\section{4}

Intracranial lateralized dermoid cyst accompanied by a subcutaneous stigmata and no dermal fistula

Selami Çağatay Önal ${ }^{1}$, Tuncay Ateș ${ }^{1}$, İclal Gürses ${ }^{2}$, Rezzan ErguvanÖnal $^{2}$, Alpay Alkan ${ }^{3}$

${ }^{1}$ Department of Neurosurgery, ${ }^{2}$ Department of Pathology, ${ }^{3}$ Department of Radiology, İnonu University School of Medicine, Malatya, Turkey

Intracranial dermoids are rare primary tumors consisting \%0.04-0.7 of the whole intracranial tumoral lesions. Typical dermoid cysts are well delineated masses without contrast enhancement compatible with lipoid density on CT and MRI. They generally evolve on the fifth/ sixth weeks of fetal life with a midline configuration. They can be seen on both ends of the evolving central nervous system most dominantly on the supra and parasellar regions and posterior fossa. The patients are usually admitted with signs of increased intracranial pressure. Convulsive fits, focal neurological deficits, and aseptic meningitis are other symptoms of admittance. A sixteen-year-old boy is admitted to the ward with the history of convulsive fits. Radiology disclosed a left temporoparietal mass accompanied by a subcutaneous stigmata with no interconnection and no dermal fistula. Total surgical excision was performed and the patient is symptomfree for seven years. A lateralized dermoid cyst with an independent subcutaneous stigmata and no dermal fistula is extremely rare.
5

Current situation of nasal encephalocele in Myanmar and activity of Japanese medical cooperative team in Myanmar

Shigeki Ono ${ }^{1}$, Masahiro Kameda ${ }^{2}$, Kiyoshi Yamada ${ }^{3}$, Yoshihiro Kimata ${ }^{3}$, Michiyasu Suzuki ${ }^{4}$, Myat Thu ${ }^{5}$, Isao Date ${ }^{2}$, Shigeru Okada ${ }^{6}$

${ }^{1}$ Department of Neurosurgery, Kawasaki Medical School, ${ }^{2}$ Department of Neurological Surgery, Okayama University Graduate School of Medicine, Dentistry, and Pharmaceutical Sciences Department of Plastic Surgery, Okayama University Graduate School of ${ }^{3}$ Medicine, Dentistry, and Pharmaceutical Sciences, ${ }^{4}$ Department of Neurosurgery, Yamaguchi University Medical School, ${ }^{5}$ Department of Neurosurgery, Yangon General Hospital, ${ }^{6}$ Myanmar Japan Collaboration Project for Fostering Medical Human Resources

Myanmar has achieved rapid economic growth, these days, because of pushing forward with its reform and open policies. In contrast, Myanmar's medical system has been behind the curve due to long closed-door policy. We have organized and supported the medical assistance, such as enlightenment of medicine, public sanitation, establishment of emergency system and medical education to Myanmar more than 15 years, separated from Japanese government's activity. These days, we, Japanese pediatric neurosurgeons had a chance of medical exchange and visitation in Myanmar through the agency of Myanmar Japan Collaboration Project for Fostering Medical Human Resources. Head and spinal trauma, infection of central nervous system, and nasal encephalocele were major concerned fields for Myanmar neurosurgeons. Owing to the fact that the total number of neurosurgeons in Myanmar is now less than 10, Myanmar's neurosurgeons have not engaged in the treatment of subarachnoid hemorrhage or brain tumors. In this country, there are many pediatric nasal encephalocele patients in contrast with its low incidence in developed countries. Therefore, the treatment of nasal encephalocele in Myanmar is not enough in the aspect of reconstruction or cosmetic reasons. In this paper we make a first report of Myanmar's current situation of nasal encephalocele, its treatment, and the future prospects of collaboration for its treatment by Japanese neurosurgeons.

6

Surgical treatment of pediatric arachnoidal cyst

Ulrika Sandvik, Bengt Gustavsson

Department of Neurosurgery, Astrid Lindgren Childrens' Hospital, Karolinska University Hospital, Stockholm, Sweden

Arachnoidal cysts are benign anomalies, most commonly identified in childhood. They have been linked to delayed psychomotorical development, seizures, headache, hydrocephalus and cranial deformation. The role of surgery and choice of surgical method remains controversial. We have conducted a retrospective review of pediatric arachnoidal cysts that have been surgically treated during the last 12 years at Astrid Lindgren Childrens' Hospital, Karolinska University Hospital, Stockholm. 43 surgically treated cases were identified. Of these 25 were arachnoidal cysts located in the temporal fossa or over the convexity, the remaining were suprasellar cysts or cystic tumors. Of the 25 arachnoidal cysts, 13 experienced long term headache whereas 11 had an acute onset of symptoms (six epileptic seizures, four with radiological signs of hemorrhage into cyst.) EEG abnormalities were detected in six children out of which five had epileptic seizures. An neuroopthalmologic examination revealed papillary edema in one child whereas three had an impaired visual field. Two children were in neuropsychological examinations found to have impaired test results The mean preoperative size (calculated by neuronavigational software) of the arachnoidal cyst was $57,5 \mathrm{ml}$ and the mean postoperative size was $26,3 \mathrm{ml}$. In the whole population open fenestration was used in 19 cases, two endoscopic fenestrations were performed and eight cystoperitoneal shunts were placed. Five patients needed reoperations using another technique. All but three children were improved in their symptoms. Of those three, two were eventually diagnosed with benign intracranial hypertension. 
Multimodal treatment of AVM's in children: 13 years single center experience

Alexandru Tascu ${ }^{1}$, Radu Eugen Rizea ${ }^{1}$, Catalin Pascal $^{1}$, Adrian Iliescu ${ }^{1}$, Liviu Nuteanu ${ }^{1}$, Fery Stoica ${ }^{2}$, Andree Chefneaux ${ }^{3}$, Radu Mircea Gorgan ${ }^{4}$ ${ }^{1} 1^{\text {st }}$ Neurosurgery Clinic- Pediatric Department; ${ }^{2}$ Gamma-knife Department; ${ }^{3}$ Neuroradiological Vascular Invasive Department; ${ }^{4} 4^{\text {th }}$ Neurosurgery Department Emergency Hospital „Bagdasar-Arseni”, Bucharest, Romania

Introduction: AVM represents $1 / 10$ of cerebral vascular malformations in USA, (1/100.000/year cases for all ages). The overall annual risk of hemorrhage is $2-4 \%$ and generally affect the young population.

Material and method: Authors present 89 consecutive cases of AVMs in children (0-16 y.o.): 44 operated (49.4\%), 32 (36\%) treated with GKS, 13 $(14.6 \%)$ by embolization and 23 cases $(25.8 \%)$, with multiple therapeutical options, in the "Bagdasar-Arseni" Hospital, Bucharest, between 20002012 (13 years). The localization of AVMs was: superficial supratentorial 65 cases $(73.1 \%)$, deep supratentorial 13 cases $(14.6 \%)$, brainstem 1 case $(1.1 \%)$ and cerebellum 10 cases $(11.2 \%)$. The most common clinical symptoms was: hemorrhage 23 cases $(25.8 \%)$ and seizures 35 cases (39.3\%). The distribution in basis of the Spetzler-Martin Grading System (1986) was: grade I 21 cases (23.6\%), grade II 27 cases (30.3\%), grade III 23 cases $(25.8 \%)$, grade IV 13 cases $(14.6 \%)$ and grade V 5 cases $(5.6 \%)$. Results: The postoperative complications were: recurrent hemorrhage 10 cases $(11.2 \%)$ and obstructive hydrocephalus in 13 cases $(14.6 \%)$. The GOS at 6 months was: good recovery 38 cases $(42.7 \%)$, moderate disability 42 cases $(47.2 \%)$, severe disability 7 cases $(7,9 \%)$, persistent vegetative state 1 case $(1.12 \%)$ and death 1 case $(1.12 \%)$.

Conclusions: Generally intracerebral hemorrhage and seizures, are the initial symptoms in AVMs in children. Microsurgery represents the best treatment in grade I-IV. The large AVMs (grade IV-V) and AVMs in functional areas requires a multimodal treatment.

Intracranial liponeurofibroma - a new entity

Suhas Udayakumaran ${ }^{1}$, M. R. Bindu ${ }^{2}$, Dilip Panikar ${ }^{1}$, Geeta Chacko ${ }^{3}$

${ }^{1}$ Division of Pediatric Neurosurgery, Department of Neurosurgery,

${ }^{2}$ Department of Pathology, Amrita Institute of Medical Sciences and Research Centre, Ponekkara Kochi, India; ${ }^{3}$ Department of Pathology, Christian Medical College, Vellore, India

Background: Intralesional fat in a neurofibroma is a rare variant of neurofibroma. Cutaneous lipomatous neurofibroma has been described. Intracranial location of the entity has not been described.

Case report: An infant, a known case congenital neurofibromatosis with buphthalmos was referred to us with magnetic resonance imaging (MRI) showing a parasellar lesion for evaluation. In view of the unclear nature of the lesion, we decided to biopsy the lesion. The lesion could be excised subtotally only, in view of the extension along the trigeminal nerve. The histopathology was described as diffuse liponeurofibroma.

Discussion: Cutaneous lipomatous neurofibroma is a lately described rare variant of neurofibroma, initially described in 2002. Clinically, lipomatous neurofibroma has been reported to be preferentially located in the head and neck. To the best of our knowledge, intracranial location of lipomatous neurofibroma has not been described.

Conclusion: We report the occurrence and describe a rare entity of diffuse liponeurofibroma in a previously undescribed intracranial location in a child with segmental orbitotemporal neurofibromatosis.

Occipital encephaloventriculocele is a Dandy Walker variant and a double hit phenomenon

Suhas Udayakumaran, A. Parasuraman

Division of Pediatric Neurosurgery, Amrita Institute of Medical Sciences and Research Centre, Kochi, Kerala
Background: Encephalocele is a complex congenital malformation. Fourth ventricle as a content occipital encephalocele has been rarely reported.

Case report: A 10-month-old infant presented to us with occipital swelling. MRI demonstrated occipital encephalocele with predominantly cystic component. Intraoperatively the cyst contained CSF with active inflow through the hiatus in the skull. It also contained a small remnant of brain parenchyma. The encephalocele was repaired. Histopathology demonstrated ependymal lining with gliotic underlying tissue.

Discussion: Encephalocele is a complex congenital malformation. Fourth ventricle as a content occipital encephalocele has been rarely reported. We noted a constant association with Dandy-Walker syndrome in literature, leading us to postulate certain characteristic prerequisites leading to the abnormality. We demonstrate the pathological anatomy in occipital encephaloventriculocele and hypothesize the embryological events leading to it.

Conclusion: Occipital encephaloventriculocele involving fourth ventricle is a part of the DANDY WALKER spectrum with constant association with vermian agenesis. The phenomenon is a double hit phenomenon with occurrence of atresia of foramen of Magendie and formation of encephalocele being the distinct events.

\section{0}

Analysis of the structure of occurrence of congenital malformations of the nervous system

Galimzhan M. Yelikbayev, A. A. Yernazarov, T. A. Maimakov,Y. A. Annaorazov

Department of the General Surgery International Kazakh-Turkish University, Turkistan, Kazakhstan

The work is based on the results of the clinical examinations and surgical treatment of the 158 children with a CM of the CNS data of the Regional Anatomical Pathology Office of the 91 death children with $\mathrm{CM}$ of the CNS in 2010-2012. From treated 158 children with CM of the CNS considerable part was patients with hydrocephalus (118-74.7\%) and spinal hernias (36-22,8\%). Children with a dermal sinus (2), cerebrospinal hernias (1) and lipoma (1) were rare. The wide rare of the patients was children to 1 year (116 patients - 73,4\%). CM of the CNS was in 447 children, accounted $20,4 \%$ for all types of the CM (447). Hydrocephalus and spinal hernia were more than half of 68 $(74,7 \%)$. Anencephaly $10(11,0 \%)$, porencephaly $6(6,6 \%)$, craniocele $5(5,5 \%)$, microcephaly and cysts of the brain to $1,1 \%$. Associated anomalies were presented from two $(20,1 \%)$, three or more defects $(79,9 \%)$. Malformations of the nervous system were found in $70 \%$ cases - in the early stages of gestation. Surgical treatment was carried out $68(57,6 \%)$ patients with hydrocephalus and in $22(61,1 \%)$ in children with spinal hernias. Postoperative mort ability in $2(2,2 \%)$ cases in children with partial dorsal rahishizis. Congenital defects of the CNS are a major cause of infant mortality and child morbidity and disability. In a structure CM of the CNS wide rade congenital hydrocephalus, spinal hernias, anencephaly.

\section{Craniofacial}

\section{1}

Fronto orbital advancement and cranial vault reshaping for children with craniosynostosis

Azmi Alias ${ }^{1}$, Mohd Ali Mohd Zain ${ }^{2}$, Ahmad Ridzuan Arsyad ${ }^{2}$

${ }^{1}$ Department or Neurosurgery, ${ }^{2}$ Department of Plastic \& Reconstructive Surgery, Hospital Kuala Lumpur, Malaysia

Introduction: Premature closure of cranial sutures resulted in restriction to the growth of underlying brain, increase intracranial pressure and 
disfigurement of skull in a young children. We describe our experience in treating 14 children with syndromic and non-syndromic craniosynostosis at Pediatric Institute, Hospital Kuala Lumpur, Malaysia.

Methods: Fronto-orbital advancement and cranial vault reshaping was performed by a combined team from Department of Neurosurgery and Plastic Reconstructive Surgery, Hospital Kuala Lumpur from June 2006 until February 2013. The approach was made through a bicoronal incision. A bifrontal flap is raised followed by exposure of the temporoparieto-occipital bone until lambdoid. Bifrontal free bone flap and fronto orbital bandeau is subsequently elevated, followed by bilateral c-shaped temporo -parietal and midline occipital strip craniectomy exposing part of the saggital sinus. The bilateral temporo-parietal bone flap is out fracture and fronto orbital bandeau is fixed to midline and bitemporal bridge with non absorbable sutures in forward and slight kyphosis position.

Results: Most of the syndromic patient achieved good cosmesis with significant improvement of nasal congestion, orbital protrusion and more active. One patient with Crouzon Syndrome developed intraoperative massive blood loss and malignant brain swelling. She was stabilized and underwent delayed reconstruction at 5 days later and discharged home after 14 days. No mortality or other morbidities.

Conclusion: Fronto-orbital advancement and cranial vault reshaping effectively allow expansion of the intracranial volume to accommodate the growing brain, reduce intracranial pressure and provide additional esthetic appearance.

\section{2}

Proteus syndrome revealing itself after the treatment of a bilateral subdural haematoma

Yassine EL Hassani ${ }^{1}$, Karl Schaller ${ }^{1}$, Benoit Jenny ${ }^{1}$, Brigitte PittetCuenod $^{2}$, Armand Bottani ${ }^{3}$, Paolo Scolozzi ${ }^{4}$, Hulya Ozsahin Ayse ${ }^{5}$, Benédict Rilliet ${ }^{1}$

${ }^{1}$ Department of neurosurgery, Geneva University Hospitals and faculty of Medicine, Switzerland; ${ }^{2}$ Plastic, Reconstructive and Aesthetic Surgery Unit, Geneva University Hospitals and Faculty of Medicine, Switzerland; ${ }^{3}$ Service of Genetic Medicine, Geneva University Hospitals, Geneva, Switzerland; ${ }^{4}$ Division of Maxillofacial and Oral Surgery, Department of Surgery, Geneva University Hospitals and faculty of Medicine, Switzerland; ${ }^{5}$ Department of Pediatrics, Hematology Unit, Geneva University Hospitals and faculty of Medicine, Switzerland

Introduction: Hypertrophy of the calvarium has different aetiologies, among them the rare Proteus syndrome.

Case report: We report the case of a young girl initially treated for relapsing right then left large chronic subdural haematoma, who progressively developed craniofacial hypertrophy consistent with the diagnosis of Proteus syndrome. Calvarium hypertrophy was shaved and remodeled combining midface advancement, essentially for cosmetic purposes. During the first calvarium remodelling, important bleeding of the bone required large volume of blood replacement. Haemostasis workup revealed platelets aggregation anomalies. Blood and fibroblast PTEN mutation was not found. Bleeding issues during subsequent surgeries were controlled with tranexamic acid and desmopressin acetate.

Conclusion: Literature review of operated patients with Proteus syndrome did not reveal an association with platelets anomalies. A complete haemostasis workup following this unexpected haemorrhagic complication is recommended for this rare pathology.

\section{3}

Post-operative pyrexia in craniofacial surgery: How frequent is it? Pasquale Gallo ${ }^{1}$, Hammad Qureshi ${ }^{1}$, Anne L. S. Elserius ${ }^{1}$, Desiderio Rodrigues $^{1}$, Guirish A. Solanki ${ }^{1,2}$

${ }^{1}$ Department of Paediatric Neurosurgery, Birmingham Children's Hospital, Birmingham, UK; ${ }^{2}$ University of Birmingham, Birmingham, UK
Objectives: To assess the frequency of post-operative pyrexia in children undergoing craniofacial surgery and to audit our clinical practise.

Design: This was a retrospective study over a 16-month period (two study periods of 8 months each), as part of completing an audit loop. Post-op pyrexia was defined as $37.5^{\circ} \mathrm{C}$ or more. Subjects 84 children ( 58 boys and 26 girls) median age of 14 months (4-120), undergoing fronto-orbital advancement and reconstruction (FOAR), calvarial remodelling or dynamic distraction surgery for craniosynostosis between 2009 and 2012 were included.

Methods: Case notes, prescription charts, observation charts and laboratory results were reviewed. All children were given three antibiotic doses peri-operatively.

Results: $60\left(27<38^{\circ} \mathrm{C}, 33 \geq 38^{\circ} \mathrm{C}\right)$ cases of pyrexia occurred in 30 $\left(12<38^{\circ} \mathrm{C}, 18 \geq 38^{\circ} \mathrm{C}\right)$ calvarial remodelling cases, $24\left(13<38^{\circ} \mathrm{C}\right.$, $\left.11 \geq 38^{\circ} \mathrm{C}\right) \mathrm{FOAR}$ cases and $6\left(1<38^{\circ} \mathrm{C}, 5 \geq 38^{\circ} \mathrm{C}\right)$ dynamic distraction surgery cases during the first three post-operative days. The incidence was highest during the second day with $61 \%(51 / 84)$. Pyrexia $\left(\geq 38^{\circ} \mathrm{C}\right)$ in posterior distraction surgery occurred in $83 \%(5 / 6)$ with OR $4.3, \mathrm{p}=0.3$ (ns). Septic screen was done in 33\% (20/60) and positive in $20 \%(4 / 20)$. Antibiotics were continued in $45 \%(9 / 20)$.

Conclusion: Pyrexia occurs in 3 out of 4 children undergoing craniofacial surgery. Posterior distraction surgery has a greater association with high temperature. Septic screens were negative in $80 \%$ (16/20) within the first 3 days and use of antibiotics beyond three peri-operative doses does not confer additional advantage.

\section{4}

Treatment of plagiocephaly by authors' operative technique Kiril D. Georgiev ${ }^{1}$, Jivko K. Surchev ${ }^{1}$, Yavor P. Enchev ${ }^{2}$, Georgi K. Kounin $^{1}$

${ }^{1}$ Department of Neurosurgery, Medical University, Sofia, Bulgaria; ${ }^{2}$ Department of Neurosurgery, Medical University, Varna, Bulgaria

Background: The unilateral coronal craniosynostosis is rare condition, which causes typical deformation of the skull: ipsilateral flattening of the forehead and orbit and contralateral bulging of the forehead. The goal of the operative treatment is to correct the deformation to aesthetically acceptable skull contour and in many cases to decompress the brain. Aim: The purpose of the study was to evaluate retrospectively the efficacy of the suggested by the authors operative technique in patients plagiocephaly.

Material and methods: During the period 1982 - 2011 year, at the Department of Neurosurgery, Medical University Sofia, 14 children with plagiocephaly were examined and surgically treated. The average age of the patients was 9.3 months and they were followed-up retrospectively. The children were operated on with different surgical techniques, 1 child was treated by the method of Matson and Shillito, another - by lateral canthal advancement (by Hoffman and Mohr. At the rest (12) patients, the authors applied their modification of the technique of lateral canthal advancement. Results: The suggested by the authors operative technique was easily and rapidly performed and corrected the skull deformation to aesthetically acceptable appearance immediately after the procedure. 1 child underwent reoperation for aesthetical improvement 7 years after the first intervention and another - after 3 years.

Conclusion: The suggested by the authors operative technique is an excellent alternative for easy and immediate correction of plagiocephaly.

\section{5}

Closure of a large frontal bone defect with brain prolapse in a 4-year-old boy with M. Crouzon: The role of preceding posterior cranial vault expansion

Alexandra Huthmann, Wolfgang Wagner

Section of Pediatric Neurosurgery, Department of Neurosurgery, University Medical Center Mainz, Germany 
A 4-year-old boy with M. Crouzon presented with a large frontal bone defect and grotesque bulging of the brain, only covered by dura and scalp. The defect was the sequel of a frontoorbital advancement performed 4 years before in another hospital. The brain bulging made the wearing of a protecting helmet necessary. The posterior aspect of the head was very flat (brachycephaly). The boy had some mental retardation, but his neurological examination was otherwise normal. He attended the kindergarten, run, jumped, rode a bicycle, played with other children continously wearing the helmet. As it was impossible to redress the brain prolapse, we performed first a posterior calvarial augmentation, giving the brain more space posteriorly. After a few weeks, it was possible to cautiously push back the bulging brain in the awake patient without causing pain or other symptoms of increased intracranial pressure. One month after the posterior expansion, a frontoorbital re-advancement was done, closing the defect with calvarial bone and a titanium mesh. Thereafter, the frontal aspect was normally flat and solid without brain bulging; the boy was allowed to freely move around without helmet. There were no symptoms of increased intracranial pressure and the patient feels comfortable (follow-up 2 years). In a very special situation with brachycephaly and brain prolapse through a large frontal calvarial defect, a preceding posterior calvarial augmentation may allow to cautiously push back the brain and to close the frontal bony defect flush with the forehead.

16

Ultrasound a sure method in scull deformities

Gertrud Kammler ${ }^{1}$, Günter Delling ${ }^{2}$, Knuth Helmke ${ }^{3}$, Manfred Westphal ${ }^{1}$, Jan Regelsberger ${ }^{1}$

${ }^{1}$ Department of Neurosurgery, ${ }^{2}$ Institute of Osteopathlogy, ${ }^{2}$ Department of Paediatric Radiology, University Medical Center Hamburg Eppendorf, Germany

Object: The diagnosis of caniosynostosis is based on characteristic head deformity, dependent to the site of suture fusion. Some cases lack the typical features, so further imaging is required to differentiate between craniosynostosis and deformational plagiocephaly. To protect infants from radiation, either $\mathrm{x}$-ray or CT-imaging, near-field high frequency ultrasound has been evaluated in the diagnosis of synostotic sutures. Methods: In an initial study synostotic sutures of 26 infants, (2 to 7 months) were investigated sonographically. Its morphologic characteristics, bony thickness and suture width were analyzed with a high frequency ultrasound system (Siemens Elegra, 7.5 MHz linear scanner) by two independent investigators. The results were correlated to the sonographic findings of 100 healthy infants and compared to CTimaging. Additionally specimens were histopathologically analyzed to illustrate sonographic findings.

Results: Hyperechogenic bridging due to osseous thickening in synostotic sutures and hypoechogenic gaps in normal sutures were highly reliable findings sonographically. Partial suture fusion was seen in 21 patients, total fusions in five. Compared to CT-imaging (15 fused sutures, 11 with partial fusion) no synostosis was missed sonographically and its resolution found to be more than two times higher than CT-imaging (0.4 vs. $1 \mathrm{~mm})$. Conclusion: Ultrasound in the diagnosis of craniosynostosis turned out to be a highly reliable examination tool preventing infants from radiation. Since this initial study a standardized protocol in diagnosing craniofacial deformities has been introduced in our daily practice confirming ultrasound to be quick, easy to handle and less expensive thereby therapeutic implications can be made by this procedure immediately.

17

Neurodevelopmental and esthetic results in children after surgical correction of metopic suture synosthosis: a single institutional experience

Mathias Kunz ${ }^{1}$, Markus Lehner ${ }^{2}$, Alf Heger $^{2}$, Lena Armbruster ${ }^{1}$, Heike Weigand $^{3}$, Gerson Mast ${ }^{4}$, Aurelia Peraud ${ }^{1}$
${ }^{1}$ Department of Neurosurgery, Ludwig-Maximilians-University Munich, Munich, Germany; ${ }^{2}$ Department of Pediatric Surgery, LudwigMaximilians-University Munich, Munich, Germany; ${ }^{3}$ Department of Pediatric Neurology, Ludwig-Maximilians-University Munich, Munich, Germany; ${ }^{4}$ Department of Craniomaxillofacial Surgery, LudwigMaximilians-University Munich, Munich, Germany

Introduction: Metopic suture synosthosis leading to trigonocephalus is considered the third most frequent single suture craniostenosis. We present herewith the clinical and esthetic results in 25 consecutive pediatric cases operated for metopic suture synosthosis with special focus on the child's neurocognitive, motor and speech development.

Methods: 25 children (age 6 to 33 months; mean $10.4 \pm 6$ months) with trigonocephaly were operated between 2002 and 2012 with frontoorbital advancement including frontal bone cranioplasty and frontoorbital bandeau remodelling. Pre- and postoperative neurodevelopmental (motor, speech, cognition) and esthetic outcome are reported.

Results: 21 (84\%) boys and four 4 girls $(16 \%)$ were included in the present study. Mean follow-up period was $33 \pm 28$ months. 2 children $(8 \%)$ were diagnosed with a syndromic. Outcome analysis for esthetic results showed a high degree of satisfaction in 23 cases (92\%). Preoperative evaluation revealed to some extent deficits in 13 children $(52 \% ; 6$ mild, 4 moderate and 3 severe deficits). 12 children ( $48 \%$ ) were proven to have a normal preoperative neurocognitive development. In eight out of ten children with mild or moderate developmental restraints improved after cranial vault reconstruction. Two syndromic children and one child with fetal valproate syndrome also improved, but neurocognitive progress was comparably slower and major deficits were still apparent at last follow-up.

Conclusions: As shown in the present study surgical remodelling for metopic suture synosthosis not only corrects esthetic appearance but also improves neurodevelopmental outcome even in syndromic forms of the deformity.

\section{8}

Percentile-based anthropometric assessment of craniosynostosis

Marcus H. T. Reinges ${ }^{1}$, Hans-Peter Howaldt ${ }^{2}$, Petros Christophis ${ }^{1}$, Jan-Falco Wilbrand ${ }^{2}$

${ }^{1}$ Department of Neurosurgery, University Hospital Giessen and Marburg $\mathrm{GmbH} ;{ }^{2}$ Department of Cranio-Maxillofacial Surgery, Plastic Surgery, University Hospital Giessen

Introduction: Pre- and postoperative assessment during craniosynostosis correction is mainly based on subjective parameters. The aim of this study was to identify differences in standardized anthropometric parameters in children with craniosynostosis in comparison to the craniofacial norm.

Methods: Anthropometric parameters of $\mathrm{n}=103$ children ( 77 male, 26 female) with different single or multiple suture craniosynostoses were compared to normative percentiles generated by anthropometric measurements in $n=401$ healthy Caucasian individuals. Preoperative cranial circumference, width, length, sagittal, and transversal hemicircumference as well as the Cranial Index were evaluated.

Results: Most of the children included in this study were scaphocephal. Cranial circumference, cranial length and sagittal circumference were above the $97^{\text {th }}$ percentile for these parameters in most cases. Cranial width and transversal circumference, however, were normal in scaphocephaly. Transversal and sagittal circumference were enlarged in trigonocephal children (mostly above the $90^{\text {th }}$ percentile), while cranial circumference tended to be below the median.

Conclusions: Although these results might not be suitable to draw definite conclusions, a percentile-based assessment of cranial deformation due to premature sutural fusion could objectify perioperative estimations. 
19

Guides to facilitate optimal directions for cranial distractor implantation

Niina Salokorpi, Willy Serlo, Juha-Jaakko Sinikumpu

Departments of Neurosurgery and Pediatric Surgery, Oulu University, Oulu, Finland

Cranial expansion with distractors has became popular. The procedure is usually safe, but complications occur such as infections and skin erosions One technical problem is the correct placement and the correct direction of the distractors. It can be challenging to place the distractors in a parallel direction on the round and sometimes asymmetric head. This especially actual in bigger children where more than two distarctors are required for proper stability. If the distarctors end up in an unparallel direction, the distractors can get "stuck" as the vectors diverge. In order to handle this, we constructed guides that can easily be fitted to the distarctor during fixation of the distarctor. The guides can be placed in either direction, or even in both directions, of the distractor. During 6 procedures during $1 / 2$ year we have used these guides, and avoided malplacement/ unparallel direction of the distractors.

\section{0}

3D-Stereophotogrammetric analysis of broad median craniectomy, forehead tilting, occipital barrel staves and green stick fracturing of temporal bone segments for correction of premature sagittal craniosynostosis

Tilmann Schweitzer ${ }^{1}$, Christian Linz ${ }^{2}$, Beatrice Jager ${ }^{1}$, Jürgen Krauss ${ }^{1}$

${ }^{1}$ Section of Pediatric Neurosurgery, Department of Neurosurgery, University of Würzburg, Germany; ${ }^{2}$ Department of Cranio,- Maxillo,-Facial Surgery, University of Würzburg, Germany

Introduction: Different methods for operation of premature sagittal craniosynostosis exist. Objective analysis of operative changes is difficult: Two dimensional methods such as photodocumentation or calipers remain two dimensional and dependant from the examiner as well as a moving child. Three-dimensional analysis performed by CT scans includes radiation exposure and the need for sedation with every examination. 3D stereophotogrammetry enables us to longitudinally analyse the course of operated but also not operated craniosynostotic children.

Methods: We analysed 20 children (mean 7.3 months, 3 female, 17 male) with isolated sagittal craniosynostosis before and after operation by $3 \mathrm{D}$ stereophotogrammetry. The operative procedure, performed at 7.3 months of age consisted of a broad median craniectomy including both proximal parts of the coronal and lambdoid suture, active forehead tilting, occipital barrel staves as well as green stick fracturing of both temporal bone segments. To align the $3 \mathrm{D}$ data sets in virtual space, a coordinate system was created based on anatomically defined reference points. Results: 3D-Analysis (Analytics 3.0 Cranioform ${ }^{\mathbb{R}}$, Alpnach, Switzerland) demonstrated a significant increase in width, CI-index, head and coronal circumference as well as intracranial volume and cranial base width postoperatively. Head length was the only parameter to decrease significantly after surgery. Parameters of asymmetry such as comparison of anterior or posterior volume quadrants and $30^{\circ}$ diagonal difference showed no significant changes.

Conclusion: Stereophotogrammetry enables a fast, radiation-free and objective analysis in all forms of skull anomalies. The concept of a broad median craniectomy and active forehead tilting brings good cosmetic results.

\section{1}

Head orthesis in positional plagiocephaly and brachycephaly

Tilmann Schweitzer, Beatrice Jager, Jürgen Krauß

Section of Pediatric Neurosurgery, Department of Neurosurgery, University of Würzburg, Germany
Introduction: Treatment options for positional skull deformities of infants' have led to ongoing discussions. While many pediatricians tend to favour positioning therapy and physiotherapy alone there is a rising number of caregivers who offer a supportive head orthesis.

Methods: We analysed 51 children (mean age 6 months) with positional plagiocephaly and 30 children (mean age six months) with positional brachycephaly before and after helmet therapy by 3D stereophotogrammetry. As control group we analysed 37 children (mean age 6.4 months) with no visible asymmetry at comparable time points. We analysed length, width, cephalic index, oblique parameters, ear shift and head circumference. By defining separate volume sections we were able to quantify the degree of asymmetry in both posterior and anterior quadrants of the head.

Results: Compared with the control group, the plagiocephalic heads remained more brachycephalic, but closely approximated the normal shape under molding therapy. The striking volume difference between the left and right posterior sections in the plagiocephalic children (the mean volume of the flattened side being $21 \%$ smaller than the one on the contralateral side) improved as well (to a residual difference of mean $8 \%$ ) and ended up with a value close to the control group (mean $6 \%$ ).

Conclusions: 3D stereophotogrammetry enables a three dimensional, objective, fast and radiation free analysis of head morphology and asymmetries. We demonstrate impressive improvements of positional plagiocephaly under molding therapy. In normal looking infants we describe the extent of unperceived asymmetry.

\section{2}

"To shunt or not to shunt" in Frontoethmoidal encephalomeningocele with intracranial abnormalities Wihasto Suryaningtyas, Muhammad Arifin Section on Pediatric, Department of Neurosurgery, Faculty of Medicine, Airlangga University, Soetomo General Hospital, Surabaya, Indonesia

Introduction: Approximately $20-25 \%$ of Frontoethmoidal encephalomeningocele (FEEM) cases harbor one or more intracranial abnormalities. In the past, almost all CSF related abnormalities were shunted. Recently, we made an effort to selectively choose the patient that would be benefited from shunting. The purpose of this study was to examine the efficacy of the decision algorithm.

Method: Medical records of FEEM patients with intracranial abnormalities were reviewed. The efficacy of the decision algorithm was examined. The primary outcome will be 1) the incidence of internal leakage or CSF related wound dehiscence. and 2) the incidence of new or progressive neurological deficit. Other outcome will be the rate or ratio of shunted and non-shunted cases.

Results: Medical record of 42 FEEM patients with intracranial abnormalities were reviewed. Male was predominate (27 vs 15 patients). The average age was 6.3 year old (ranging from 6 months to 23 years old). Types of intracranial abnormalities found were hydrocephalus ( 9 cases), arachnoid cysts and porencephalic cyst (20 cases), ventricular malformation (25 cases), agenesis of carpus callosum (4 cases), and agenesis of septum pellucidum ( 5 cases). We placed a shunt along with the repair of FEEM on 7/42 patients. No leakage was observed for these patients. No new or progressive neurological deficit was noted on all patients.

Conclusion: The decision algorithm for placing a shunt in FEEM with intracranial abnormalities is proven effective in reducing complication and avoiding unnecessary burden from shunt for those without the need for a CSF diversion.

23

Minimally invasive spring-assisted cranioplasty for sagittal suture synostosis

Marie-Lise C. van Veelen ${ }^{1}$, Caroline Touw ${ }^{1}$, Irene M. Mathijssen ${ }^{2}$ 
${ }^{1}$ Department of pediatric neurosurgery, Erasmus University Medical Centre Rotterdam; ${ }^{2}$ Department of Plastic and reconstructive surgery, Erasmus University Medical Centre Rotterdam

Introduction: Correction of sagittal suture synostosis has a cosmetic and functional purpose. Minimally invasive spring-assisted cranioplasty aims to address both purposes.

Methods: The results of the first 59 patients treated with this technique at our institution, between January 2010 and December 2012, have been analysed. The technique consists of two small incisions perpendicular to the sagittal suture. These incisions provide sufficient access to perform two parasagittal craniotomies and place two distractive springs.

Results: Cephalic index (CI) at presentation was 67 (sd 3.9), after surgery $\mathrm{CI}$ increased to 75 (sd 4.3), at one year after surgery CI decreased to 74 , after that CI remained stable at 74 (sd 3.3). Head circumference (HC) decreased from $1.8 \mathrm{sd}(\mathrm{sd} 1.5)$ at presentation to $0.6 \mathrm{sd}(\mathrm{sd} 0.9)$ one year after surgery, it than remained stable. Mean blood loss was $78 \mathrm{ml}$ (sd 83). One patient had a dural tear and one patient had insufficient distraction due to an incomplete craniotomy.

Conclusion: Spring-assisted cranioplasty requires only two small incisions and is at least as effective as other techniques with respect to the cephalic index. Head circumference is comparable to other techniques as well. Blood loss, operative time and complication rate are reduced. Disadvantages are the need to remove the springs in a second intervention and the fact that expansion of the spring is not controllable after placement. This requires careful planning to adjust the spring and/or the craniotomy to the patient's specific features.

\section{Dysraphism/Tethered Cord}

\section{4}

Heteropagus twin removal using intra-operative monitoring: A case report

Azmi Alias ${ }^{1}$, Zakaria Zahari ${ }^{2}$, Fadzlishah Johanabas Rosli ${ }^{3}$

${ }^{1}$ Consultant Neurosurgeon, Neurosurgery Department Hospital Kuala Lumpur; ${ }^{2}$ Consultant Paediatric Neurosurgeon \& Head of Department, Paediatric Surgery Department Hospital Kuala Lumpur; ${ }^{3}$ Neurosurgery Registrar, Neurosurgery Department Hospital Kuala Lumpur

Introduction: Heteropagus-or parasitic-twins, are conjoined twins where the severely defective twin is dependent on the intact twin for survival. The estimated incidence is about 1 in 1 million live births. Pygopagus twinning is a spectrum where the twins are conjoined in the lumbosacral region. We present a case of a baby girl with an accessory lower limb in association with spinal dysraphism, and the successful separation of the heteropagus twin with the help of intra-operative neurophysiology monitoring.

Method: The child was born with a fully-formed and autonomously moving accessory lower limb on her back, with three other primitive appendages. The surgery was aimed at removing the heteropagus twin without damaging the intact twin's lower limbs, and bladder and bowel functions. This was achieved with intra-operative monitoring of these target organs' skeletal musculature using electromyography (EMG). The child had lumbosacral dysraphism and myelomeningocele, and the accessory limb received its own innervation at the level of the right anterior tibialis. The nerve was excised without compromising the intact twin's nervous system, and the myelomeningocele was successfully repaired. Results: The heteropagus twin was successfully removed en bloc, and the surgical wound was closed primarily, with preservation of the surviving twin's lower limbs and bladder and bowel functions. The surgery was complicated with wound breakdown, but with vigilant care, the patient was eventually discharged well.

Conclusion: Intra-operative neurophysiology monitoring played an important role in preserving the surviving twin's nervous functions, as we were able to delineate the innervation of the accessory limb.

\section{5}

Tethered cord syndrome and occult spinal dysraphism. Clinical features and surgical results

Oscar Garcia Gonzalez

Division of Pediatric Neurosurgery, Hospital Regional de Alta Especialidad del Bajio, Leon, Mexico

Introduction: Progressive neurological deterioration localized to the lower spinal cord resulting from traction on the conus medullaris has been termed TCS. Features associated with the syndrome include the dysraphic spine, cutaneous stigmata, vertebral-orthopedic abnormalities, neurological deterioration of the lower spinal cord including bowel and bladder dysfunction. Mechanical traction on the spinal cord may be a cause of progressive symptoms appearing after hypoxic damage within the conus medullaris.

Patients and methods: The clinical features and outcome in 14 pediatric patients whit TCS treated surgically at the Hospital Regional de Alta Especialidad del Bajio between 2009 - 2012 was studied retrospectively. 9 children with spinal lipomas and closed spinal dysraphism, 2 children whit dermal sinus, one of them infected, 1 children whit myelocistocele, and 2 children whit tight filum terminale were included.

Results: During the period in 7 boys and 7 girls aged from 2 months to 4 years (mean age 12.9 months) detethering procedures were performed. Most of the patients were asymptomatic prior to surgery and $20 \%$ of the patients presented whit neuro-orthopedic syndrome and urinary dysfunction. CSF collection, occurred in 3 cases and infection in 2 cases; none of these complications caused permanent morbidity. Of all signs and symptoms, muscle weakness and bladder dysfunction responded most favorably to surgical treatment.

Conclusions: Prophylactic detethering appears to be effective among asymptomatic patients. Early surgical detethering seems to be more effective in improving neurological symptoms. Once symptomatic, the surgical detethering in TCS patients tend to stop further progression of symptoms and even improved.

\section{6}

Outcome of spinal cord untethering surgery in adults

Holly S. Gilmer, Sonja C. Young

Oakland University, William Beaumont School of Medicine, Royal Oak, MI, USA

Adult spinal dysraphism involves occult tethered spinal cord as well as tethered cord related to known spina bifida (SB). Over the last 5 years, 23 patients over 21 years of age have undergone initial spinal cord untethering at our institution. Their ages ranged from 21 to 52 at the time of surgery, with an average age of 32.2 years. The group consisted of 13 females and 10 males. Eight patients had known SB, and the other 15 patients had newly-diagnosed tethered spinal cord. The most common presenting complaint was back pain $(n=22)$, although the same number of patients also had symptoms of neurogenic bowel and/or bladder. Presenting symptoms also included headache, neck pain, and upper and lower extremity weakness. Length of follow-up currently ranges from 3 weeks to 3 years, 9 months. Back pain and neck pain resolved or improved in all cases $(100 \%)$. Bowel and bladder dysfunction resolved completely in 17 cases $(74 \%)$. Strength improved in 16 patients $(70 \%)$. One of the known SB patients had transient lower extremity weakness, which resolved within a week. Another of the known SB group developed numbness in her feet, which has not resolved yet (6 weeks post-op). Other complications were 2 pseudomeningoceles requiring reoperation for repair $(9 \%)$, and 1 wound dehiscence with infection (4\%). Our results suggest that spinal cord untethering in adults is beneficial in relieving symptoms, even those that may be long-standing. In experienced hands, spinal cord untethering may be accomplished in adults without unacceptable risk as well. 
27

Tethered cord in adulthood: Surgical outcomes of lipomyelomeningocele in an fourteen patients

Flavio Giordano, Stefano Ricci, Barbara Spacca, Michele Parolin, Regina Mura, Luigi Sardo, Mirko Scagnet, Federico Mussa, Pierarturo Donati, Massimiliano Sanzo, Lorenzo Genitori

Department of Neurosurgery, "Anna Meyer" Pediatric Hospital, Firenze, Italy

Lipomyelomeningoceles (LMMs) are usually diagnosed and treated in childhood though their surgical indication is still debated. Late surgery in adults with symptoms and signs of tethering cord syndrome is rare but sometimes performed. Since 1994 to 2013 fourteen symptomatic adults older than 16 years old with LMMs underwent spinal untethering: 4 males, 10 females; mean age 3,3 years (17,5-57,2 yrs). Clinical findings were lower limb weakness (9) and hypoesthesia (6), chronic back and leg pain (7) bladder dysfunction (7), fecal incontinence (4). Six subjects had been submitted to previous surgery in younger age. All patients received spinal untethering with application of artificial dural substitute. Mean follow-up was 15,7 months (5-45 mos). Seven patients improved mostly in pain (6/7), followed by motor weakness (6/9), and bladder dysfunction (3/7). Four patients did not refer any amelioration after surgery. Three patients worsened after surgery because of bladder dysfunction (3), motor impairment (2), fecal incontinence (1) and pain (1). Surgical morbidity occurred in one patient because of CSF leak that needed surgical repair. Two patients showed late retethering syndrome during follow-up. Late surgery of LMMs in adults seems to be mostly efficacious in control of pain and improvement of motor function. Chronic and long-lasting urological impairment may be stabilized but not improved though worsening must be considered. Altough the number of adults is not comparable, surgical morbidity in this series $(27.3 \%)$ is higher than in the series of pediatric LMMs of the same institution (13\% in 61 operated cases).

\section{8}

Neurosurgical treatment of Diastematomyelia in a 2-year-old child Miroslav Gjurasin ${ }^{1}$, Goran Krakar $^{2}$, Davor Bojic ${ }^{3}$, Vlasta Djuranovic ${ }^{2}$, Tonci Grmoja ${ }^{4}$, Josip Marjanovic ${ }^{4}$, Ljiljana Popovic ${ }^{5}$, Djurdjica Moscatello ${ }^{5}$

${ }^{1}$ Department of Neurosurgery, ${ }^{2}$ Department of Neuropediatrics, ${ }^{3}$ Department of Pediatric Orthopedics, ${ }^{4}$ Department of Pediatric Radiology, ${ }^{5}$ Department of Pediatric Anesthesiology, Children's Hospital Zagreb, School of Medicine University of Zagreb, Zagreb, Croatia

We present the treatment of a 2-years-old female child with diastematomyelia, or cleft spinal cord. Neuropediatric and orthopedic assessment were performed because of progressive walk deterioration and trunk asymmetry. MR of spinal canal revealed split spinal cord at L1L3 level, with two separated hemicords each in separate dural and arahnoid sacs, with midline bony septum at L3 level, that completely divided spinal canal. Low position of medullary conus at S1 level was associated with tight filum. We performed L1-L2 osteoplastic laminotomy and L3 laminectomy. After bony septum removal and dural opening, we exposed cleft spinal cord with two hemicords. The caudal end of dural septum was found to be in immediate contact with the distal spinal cord exactly at the site of reunion of two hemicords, i.e. distal L3 level. This was the point of spinal cord stretching. The cranial end of dural septum was not in contact with the proximal spinal cord at the point of splitting into two hemicords. Instead, the firm fibrous midline subdural band extended from the cranial end of the dural septum at the cranial level L3 toward cranially, between two hemicords, and was firmly attached to inner dural layer at the level L1. The septum and band were removed, and the tight filum identified and resected through a small additional flavectomy at L5S1 level, allowing the distal spinal cord to be slightly released toward cranially. We present intraoperative images, analyze operative approach, and emphasize possible pitfalls that we detected performing this rare operation.

29

A distinctive rare dysraphism with mostly favourable prognosis: limited thoracic myeloschisis

Amin Hashemi ${ }^{1}$, Angelika Seitz ${ }^{2}$, Michael Elsässer ${ }^{3}$, Joachim Pietz ${ }^{4}$, Andreas Unterberg ${ }^{1}$, Heidi Bächli ${ }^{1}$

${ }^{1}$ Clinic of Neurosurgery, University Hospital, Heidelberg; ${ }^{2}$ Clinic of Neuroradiology, University Hospital, Heidelberg; ${ }^{3}$ Department of Gynecology and Obstetrics, University Hospital, Heidelberg; ${ }^{4}$ Department of Pediatric Neurology, University Hospital, Heidelberg

Objective: Limited dorsal myeloschisis (LDM) is a rare and distinctive form of spinal dysraphism. It is most frequently located in the lumbar segments with an incomplete segmental closure of the neural folds and without an overtly exposed neural plate.

Methods: We describe two cases of segmental thoracal myelochisis. The first case (one day old male) shows a distinct nonsaccular skin lesion on his back after birth with no neurological deficits. Intrauterine ultrasound was uneventful. Postpartal MRI showed a limited dorsal myeloschisis with tethering of the spinal cord at the site of the stalkcord junction (TH 5/6). The second case (15 months old female) was born with a right hemiparesis and Horner's syndrome. On MRI an extrapleural meningocele in continuity with the thecal sac and a diastematomyelia was seen with a neural stalk connecting the spinal cord ventro/laterally.

Results: The first infant was operated soon after birth with an untethering of the spinal cord and resection of the fibroneural stalk without postoperative neurological deficits. The second infant was not yet operated as no significant tethering could be diagnosed and expected improvements of neurological deficits were limited.

Conclusions: Limited myeloschisis is a less extreme form of primary neurulation failure. Distinction to other pathologic entities such as myelomeningocele and early diagnosis is important as outcome and surgical procedure may be quite different and unnecessary abortions could be avoided. We propose furthermore to modify the definition of limited dorsal myeloschisis to include also a ventro-lateral form of this entity.

\section{0}

Adult thoracic myelomeningocele without neourological deficits Vaner Köksal

Recep Tayyip Erdogan University School of Medicine, Department of Neurosurgery, Rize, Turkey

Introduction: Spina bifida is the most frequently encountered congenital anomaly of pediatric neurosurgery. The most severe form is myelomeningocele. According to Shore et al., 3.7\% of aperta sipina bifidas have been encountered in cervico-thoracic region. Myelomeningocele has been no doubt is the most frequently encountered and slow progressing form of the congenital malformations compatible with life.

Case Report: In 20-year-old male case, the hard sac in the brainstem had no neurologic deficit except from the cosmetic bad appearance. The patient described that he experienced some electrifications spreading through up and down when hitting somewhere over the sac. On MR images of the case, there were bands extending from spinal cord to skin within the myelomeningocele sac. Because of those findings, the patient was decided to be operated.

Discussion: In general, this malformation cord ended upon the skin surface. A thin fragile skin covers upon them. However, in the case we presented, it has been covered with a thick skin in contrast to this and there was no BOS fistula formation probability upon the sac.

Result: Dysfunctional neural tissue was seen in myelomeningocele sac apex during the surgery of the case. Fibrotic bands extending the location 
of the apex of the sac was resected. The base of the sac fully dissected. For the pathologic analysis, it was evaluated in accordance with the neural supporting tissue. Thoracic myelomeningocele case that grew in maturity without neurologic sequel has been desired to be presented with different radiologic images.

\section{1}

Is it congenital? Is it a result of the tethered cord during the growth? Adult lumbar block vertebrae

Vaner Köksal ${ }^{1}$, Ibrahim Suat Öktem ${ }^{2}$

${ }^{1}$ Recep Tayyip Erdogan University School of Medicine, Department of Neurosurgery, Rize, Turkey; ${ }^{2}$ Erciyes University Medical Faculty, Department of Neurosurgery, Kayseri, Turkey

Introduction: Tethered cord is a syndrome characterized with conus medullaris ending under L2 level, thickened fatty filum terminale and neurologic deficits. As well as it can be unaccompanied, it can generally be accompanied by midline closure anomalies such as lipomyelomeningocele, split cord malformation and dermal sinus. Whereas midline closure anomalies have been the most common reason for tethered cord syndrome in childhood, intradural masses, thickened fatty filum terminale and myelomeningocele operations that have been had before have been the reasons for adults. Scoliosis in $25 \%$ rate occurs especially in spinal vertebrae. During the growth stage, spinal vertebrae has been accepted as occurring through the effort of adaptation to the tethered cord.

Case report: On magnetic resonance images of the 57-year-old female case obtained through lumbar pain complaints, split cord malformation on $3^{\text {rd }}$ lumbar vertebrae level and tethered cord were visualized. There was no scoliosis in physical examination. However, lumbar $3^{\text {rd }}$ and $4^{\text {th }}$ vertebrae corpuses were determined as fusion.

Discussion: In our clinical experiences, congenital fusions in the spine or block vertebra appearance have frequently observed in cervical region in Klippel-feil syndrome. There has been no congenital fusion in lumbar region of vertebrae in the literature. Moreover, if it is not congenital, it can be evidence related to its occurrence because of the strain in phylum on sagittal plane with a mechanism similar to scoliosis in vertical axis accompanying to the tethered cord. We have desired to present this case that we have considered as being a different spinal deformity.

\section{2}

Neurosurgical aspects of urinary bladder reinervation in spina bifida patients

Zdenek Mackerle $^{1}$, Eva Brichtova ${ }^{1}$, Pavel Zerhau ${ }^{2}$

${ }^{1}$ Department of Pediatric Neurosurgery, ${ }^{2}$ Department of Pediatric Urology, The University Hospital Brno, Brno, Czech Republic

Neurogenic bladder dysfunction in spina bifida patients represents a major medical and social problem. The actual research is rather casually focused on electric stimulation or modulation of pelvic organs. A significant progress was achieved by stimulation of sacral spinal cord ventral roots (SARS). Another promising alternative of micturition is reflex arc remodelling (artificial skin-CNS-bladder pathway), firstly mentioned by Xiao. Authors would like to compare several methods of reinervation of the urinary bladder with emphasis on the Xiao procedure. The pros and cons are figured and the pitfalls of such challenging surgery are accented. The data are drawn from the running animal experiment. The most promising method of the urinary bladder reinervation is considered the co called Xiao procedure. The proper surgical procedure has to be electrophysiologically controlled and necessitates the experienced microsurgical technique, but it is definitely possible to suture the spinal nerve roots as to create the functional anastomosis. The main controversy is the real practical outcome, how powerful spontaneous micturition can one anticipate after the artificial stimulus. The loss of motion in the (hinder) leg is not so significant in animals in our experiment but could be of greater importance in previously disabled children. The artificial reflex arc remodelling for the neurogenic bladder dysfunction is a great microsurgical challenge with very promising aspects. On the other hand there is still several unanswered questions concerning the surgical technique and especially the clinical outcome.

Financial support: IGA MZ CR NT13871-4

\section{3}

Surgical treatment and outcome in patients with myeloschisis

Hirokazu Nakatogawa, Takamichi Yamamoto, Kyo Togasawa, Ayataka Fujimoto, Chikanori Inenaga, Tokutaro Tanaka

Department of Neurosurgery, Seirei Hamamatsu General Hospital, Shizuoka, Japan

Rationale: Spina bifida with myeloschisis is the most severe form in these malformations, and occasionally gives us difficult situations with complications. Then myeloschisis is always a challenging issue for pediatric neurosurgeons. Our surgical approach and complications in treatment of myeloschisis are reviewed in cases of our hospital.

Methods: Nine neonates with myeloschisis were surgically treated from January 2008 through March 2013. The outcome and perioperative complications were reviewed retrospectively.

Results: Eight patients underwent a ventriculo-peritoneal (V-P) shunting procedure before or after the repair of myeloschisis. Among these patients, four of them were treated with a ventricular access device (VAD) before V-P shunting, and the others took delayed shunting without a VAD. All of eight patients did not show any infection of V-P shunting systems. However, one patient demonstrated shunt malfunction and then revision of the system was necessary. Two patients got wound dehiscence and underwent repair with debridement. Eight out of nine cases (88\%) have patency of the V-P shunting system.

Conclusion: VADs may not be necessary before V-P shunting in patients with myeloschisis. We do not regularly place VADs at this moment and wait for about two weeks to see an exact timing of V-P shunting. Skin closure at the end of surgical procedure is sometimes difficult because of a large defect of the skin after myeloschisis repair, and may need participation of plastic surgeons to get a better outcome.

\section{4}

Wound care in children operated for tethered cord syndrome Farideh Nejat ${ }^{1}$, S. Shahjouei ${ }^{1}$, Mostafa El Khashab ${ }^{2}$

${ }^{1}$ Children Hospital Medical Center, Tehran University of Medical Sciences, Tehran, Iran; ${ }^{2}$ Hackensack University Medical Center, New Jersey, US

Background: Tethered cord includes several congenital abnormalities like simple tethered cord syndrome with thick filum, lipomyelomeningocele, split cord malformation and dermal sinus tract. Postsurgical CSF leak, wound dehiscence or subcutaneous CSF collection is a serious problem but there is no definite protocol to decrease the risk of CSF leakage. Method: This is a randomized clinical trial performed in Children Hospital Medical Center in Tehran. We have considered four protocols to evaluate their effects on the wound care which includes prone position, acetazolamide both of them and finally none of them were considered. Result: During 2 years 120 patients with tethered cord syndrome were operated for cord untethering that were included in one of four groups randomly. They were aged from 1 month to 13 years at the time of operation. All surgeries have been done with one neurosurgeon aiming good coverage of neural tissue in five layers, watertight dural closure with or without patch graft and good facial closure. Routine pressure dressing was used in all patients. Protocol failure was considered if wound dehiscence, leakage or CSF collection needed tapping was observed. All patients were followed at least for 3 months. The data of all four groups were collected and compared. 
Conclusion: Wound problem including dehiscence and CSF leakage are serious problems subsequent to tethered cord surgery. There is no established protocol to prevent these complications. Most surgeons manage the wound according to their experience. We hope this study can find an answer to this problem.

\section{5}

Tethered Cord Syndrome - with special emphasis on normal level conus and filum terminale with normal appearance

Mehmet Selçuki ${ }^{1}$, Ahmet Sükrü Umur ${ }^{1}$, Mustafa Barutçuoglu ${ }^{1}$, Nurcan Umur $^{2}$, Mesut Mete ${ }^{1}$, Deniz Selçuki ${ }^{3}$

${ }^{1}$ University of Celal Bayar, School of Medicine, Department of Neurosurgery; ${ }^{2}$ Institute of Health Sciences, Celal Bayar University; ${ }^{3}$ University of Celal Bayar, School of Medicine, Department of Neurology, Manisa, Turkey

Among 213 tethered cord patients, 68 patients with conus medullaris at normal level and without accompanying spinal developmental malformations were included in this study. Symptoms and signs, findings in investigations and observations during surgical procedure were evaluated. The results of the evaluation are discussed and compared with related literature with special emphasis on normal level conus medullaris and filum terminale with normal appearance. Consequently three main presentations of tethered spinal cord have been noticed. They are namely incontinence of urine, scoliosis and perineal/sciatic pain. After all, we have concluded that tethered spinal cord patients had benefit from untethering surgical procedures while pain as a symptom, was noticed to be taken advantage of the intervention mostly.

\section{6}

Dermal sinus tract of the spine. Clinic experiences in 13 year Mehmet Selcuki, Ahmet Sukru Umur, Seymen Ozdemir, Mustafa Barutcuoglu, Mesut Mete, Yusuf Kurtulus Duransoy, Nurcan Umur Department of Neurosurgery, Celal Bayar University School of Medicine, Manisa, Turkey

Objective: Spinal dermal sinus tract(DST) is a rare entity in the neural tube closure defects which usually comes to clinical attention by cutaneous abnormalities, neurologic deficit, orthopedics anomalies and infection. We performed this study to assess presentation patterns, associated anomalies, radiologic, urologic, hystopathological aspect and surgical findings in 15 childrens afflicted with spinalDST in our clinic follow-up 15 years experiences.

Methods: Medical records of 15 childrens treated for spinalDST in our clinic from 2000 to 2013 were reviewed.

Results: Patients ages ranged from 10 days to 36 years. Dermal sinuses were located most frequently in the lumbar and lumbosacral regions. The most common causes for referral were abnormal skin and associated anomalies examples orthopedics findings and infection. The most common magnetic resonance imaging findings were tethered cords. All patients underwent complete resection of the tract and repair of associated abnormalities.

Conclusion: Dermal sinus tract is an innocuous-appearing spinal dysraphism that may contribute to devastating morbidities if not timely addressed. In order to prevent complications, timely surgical intervention including complete resection of sinus tract with intradural exploration and correction of associated abnormalities is of utmost importance. If any symptom is observed surgery should be done as soon as possible. For asymptomatic sinuses identified by the pediatrician during a regular visit prophylactic surgery is recommended as soon as the child can tolerate operation.

There are a few large series in the literatures Radhamanesh F(2010) 35 cases, Ackermann LL (2003) 28cases, Elton S (2001)23 cases, Jindal A (2001) 23 cases. We present in this study to you 15 cases within $15 y$ years with 15 cases.
37

Tethered cord syndrome associated with diplomyelia and myelomeningocele manqué: Case report

Ozkan Tehli, Yusuf Izci, Ilker Solmaz, Cahit Kural

Department of Neurosurgery, Gulhane Military Medical Academy, Ankara, Turkey

Although tethered cord syndrome (TCS) is rare in childhood, some associated malformations may be seen in these patients. The aim of this report is to present a case of pediatric TCS associated with myelomeningocele manqué ve diplomyelia. 10-year-old female child presented with hypertrichosis, red skin color in the back and weakness and numbness in both legs. After the radiological evaluation, diplomyelia at L1 level and myelomeningocele manqué at L2 level was determined. The spinal cord was ended at L4 level. The child underwent surgical treatment and laminotomy between L1-L5 was performed. The fibrous band at L1 level was cut and the myelomeningocele manque at L2 was repaired. The filum terminale was also cut and the spinal cord was released. There was no complication in the postoperative period and the complaints of the patient was improved gradually after surgery. The children with TCS should undergo a detailed radiological examination and all associated anomalies should be detected. The surgery should be planned in order to repair all malformations which are associated with TCS. Otherwise the neurological findings did not resolved after an inappropriate surgery.

\section{8}

Diagnostic results of spinal hernia in children under 1 year

Galimzhan M. Yelikbayev

Department of the General Surgery International Kazakh-Turkish University, Turkistan, Kazakhstan

The relatively high detection of spinal hernia, clinical severity and poor treatment outcomes for a number of its forms make the problem of diagnosis and treatment to date and today. Work based on the results of the clinical examination of 30 children with the spinal hernias. Age of patients ranged from 1 day to 1 year. Children from birth to 1 month amounted to $-17(56,6 \%)$. Among patients with spinal hernias most were children with meningomyeloradikulocele (63.8\%). Hernias often had a wide base and a leg, so that they hung down and were like hanging (73.1\%) As the child's size herniation increased. And some children slowly (6), others more rapidly (24). Integuments herniation in $93.3 \%$ of cases were thin, strained and combined with hydrocephalus. Sometimes there is a maceration of the skin due to constant friction of clothing, joined inflammatory changes $(26.6 \%)$. Lower flaccid paraparesis occurred in $63.3 \%$ of cases, and paraplegia occurred in $16.6 \%$ of cases. More than $70 \%$ of patients with myelomeningocele arose combined incontinence. In children younger than 1 year at the spinal hernias observed neurologic lower extremities and the combination with other anomalies and malformations Improved results of treatment of hydrocephalus has been the determining factor of long-term positive results of treatment of spinal hernias in children.

\section{9}

A modern approach to the diagnosis and treatment of the children with congenital dermal sinus

Galimzhan M. Yelikbayev

Department of the General Surgery International Kazakh-Turkish University, Turkistan, Kazakhstan

The dermal sinus refers to congenital deformities, representing one of the subtypes of the "closed" spinal dysraphism. The dermal sinus is rare the only form of spinal dysraphism. Analyzed the results of clinical examination and surgical treatment of 24 children with congenital dermal sinus. Congenital dermal sinus in $91.7 \%$ was observed in the lumbosacral and $1 /$ 3 of the patients ranged in age from 7 to 11 years. In $83.3 \%$ of cases met 
cutaneous manifestations of disease. In 5 cases of dermal sinus combined with diastematomyelia, two observations with a lipoma. Movement disorders manifested flaccid paraparesis lower $(58.3 \%)$, tetraparesis $(8.3 \%)$ with hypostasis lower extremities $(25 \%)$. Violation of the pelvic organs were detected in $50 \%$ of children with $66.7 \%$ of patients had orthopedic disorders in the form of curvature of the spine, clubfoot with gait disturbance. In $91.7 \%$ of patients carried out an MRI study. Surgery was performed in $95.8 \%$ of children. After surgical treatment have improved in $81.8 \%$, stabilization - at $18.2 \%$, deterioration in one case. In $70.8 \%$ of children regress occurred in first week after surgery. Neurological symptoms in patients with dermal sinus is due to three causes: congenital dysplasia of the spinal cord, the presence of space-occupying lesions in the spinal canal and inflammation. In children with congenital dermal sinus before surgery should be comparing the clinical and electrophysiological data, will determine the optimal amount of the operation. Surgery in children with congenital dermal sinus is effective when carried out under endoscopic control with magnifying optics and endoscopy.

\section{0}

Prenatal correction of spina bifida using a minimally invasive (fetoscopic) approach: case report

Nelci Zanon ${ }^{1}$, D. A. L. Pedreira ${ }^{2}$, R. M. Sa ${ }^{3}$, G. L. Acacio ${ }^{4}$, E. C. Ogeda ${ }^{5}$, A. I. B .S. Dias ${ }^{6}$, T. B. Uras

Fetal and Perinatal Center, Samaritano Hospital, Sao Paulo; ${ }^{3}$ Federal University of Rio de Janeiro; ${ }^{4}$ Taubate University, Sao Paulo

Introduction: The antenatal correction of MMC has become the gold standard after the publication of the MOMS study. The endoscopic correction has been studied as a lower maternal risk alternative.

Case report: OFW, 33-year-old, pregnant first child, planned pregnancy but no folic acid used. Fetal lumbosacral myelomeningocele (L5 to S1) with cerebellar herniation was diagnosed at 25 weeks. Fetal karyotype was normal and fetal surgery was performed at 27 weeks. General anesthesia was used and three trocars were ultrasonographically placed. After partial $\mathrm{CO}_{2}$ insuflation of the uterine cavity and fetal positioning, the skin around the $3.0 \times 2.0 \mathrm{~cm}$ defect was dissected and the transition zone was excised. A dural patch was fitted to cover the wound, and was sutured to the adjacent normal skin. $\mathrm{CO}_{2}$ was removed and the uterine cavity was washed, and refilled with warmed saline. After 3 days she was discharged. After 14 days, premature rupture of membranes occurred, but Ultrasound and MRI showed upward displacement of fetal cerebellum. She was hospitalized for one week, and cesarean section was performed at $30 \mathrm{w} 4 \mathrm{~d}$. The male, $2600 \mathrm{~g}$ newborn was submitted to immediate correction of a small meningocele (without cord or nerve roots inside) at the point of patch's suture dehiscence. Postnatal MRI showed the cerebellum above the foramen Magnum and motor assessment 1 level below the lesion (sacral). He was discharged after an aneventfull neonatal outcome.

Discussion: In Germany, 80 patients have already undergone endoscopic correction. The neonatal outcomes have been similar to the MOMS study. This was the first case of endoscopic correction done outside of Germany. The technique looks promising, but long term outcome are essential to draw any conclusions.

\section{Epilepsy}

\section{1}

Temporal lobectomy to manage intractable temporal lobe epilepsy in children

Mostafa El Khashab, Arno H. Fried

Hackensack University Medical Center, Hackensack, New Jersey, United States

Standard temporal lobectomy developed parallel to finding temporal lobe epilepsy in patients with intractable seizure and electrophysiologic, neurologic, neuropsychological, and imaging data that indicate temporal lobe as the origin of the seizures. The preoperative work-up and patient selection process should be carried out at center experienced in surgical treatment of epilepsy. Our series of 12 pediatric patients with intractable seizure is reported here. Patients aged from 1.5 to 21 years (mean=13.7), without any sex predominance. We used subdural grid in $67 \%$ of our patients. Craniotomy was performed and anterior temporal lobectomy was done in 6 patients in right and 6 in left sides. Hospitalization period was 5-56 days (mean=25). Wound swelling, subdural hematoma and seizure were found in $2,1,1$, patients respectively. Seizure was controlled very well in most patients. Overall, patients with medically resistant TLE are better managed with epilepsy surgery rather than continued medical treatment. Temporal lobectomy is well established as a safe and effective treatment for medically intractable TLE.

42

Morphological and functional changes in the human hippocampus in temporal lobe epilepsy

Thomas M. Freiman ${ }^{1}$, Kathrin Leicht ${ }^{1}$, Michael Frotscher ${ }^{2}$, Vera van Velthoven ${ }^{1}$

${ }^{1}$ Department of Neurosurgery, University Medical Centre, AlbertLudwigs-University, Freiburg, Germany; ${ }^{2}$ Department of Neuroanatomy, Institute of Anatomy and Cell Biology, Albert-Ludwigs-University, Freiburg, Germany

Temporal lobe epilepsy is characterized by a sclerosis of the hippocampus (Ammon's horn sclerosis) with a specific loss of pyramidal cells in CA1 and mossy cells in the hilus. Excitatory granule cells survive, but show a reorganization of their axonal connections. Their axons, the mossy fibres, lose their target cells due to mossy cell death and sprout backwards into the granule cell layer. It was suggested so far, that this leads to a recurrent, excitatory circuit. The aim of this study was to examine the target cells of mossy fibre sprouting, and determine whether mossy fibres impinge only on granule cells or also on other neurons, in particular inhibitory interneurons (basket cells). Mossy fibres were traced with neurobiotin. In addition double immunohistochemistry against synaptoporin (mossy fibres) and parvalbumin (basket cells) was used. Synapses were examined with electron microscopy, labelled in addition with post-embedding gamma-aminobutyric-acid (GABA)-immunogold. In human epileptic hippocampi sprouted mossy fibres innervated not only excitatory granule cells but also inhibitory interneurons. In addition, we show that the inhibitory axonal plexus around granule cells is preserved and that the number of inhibitory axon terminals exceeds the number of excitatory sprouted mossy fibre terminals on granule cells. Sprouting of mossy fibres does not simply results in an excitatory circuit of granule cells because recurrent mossy fibres also innervate inhibitory interneurons. This might lead to increased inhibition and synchronization of granule cells because the extensive inhibitory axonal plexus is preserved and shows an additional innervation through sprouted mossy fibres.

\section{3}

Intraoperative continuous interictal epileptiform discharge rate during functional hemispherotomy in children

Benoit Jenny ${ }^{1}$, R. Tyrand ${ }^{1}$, Shahan Momjian ${ }^{2}$, Yassine El Hassani ${ }^{2}$, M. Seeck ${ }^{1}$, Karl Schaller ${ }^{2}$, C. Boëx ${ }^{1}$

${ }^{1}$ Department of Neurology, ${ }^{2}$ Department of Neurosurgery, University Hospitals of Geneva, Geneva, Switzerland

Introduction: Functional hemispherotomies represent are effective to treat intractable epilepsy. Changes of epileptic discharges during surgical disconnection are poorly understood.

Goals: We describe here the effects of surgeries' step on interictal epileptiform discharges (IEDs) and whether changes in IEDs rates (IEDs/min) 
could be used as intraoperative neurophysiological markers of surgical efficiency.

Methods: Intraoperative electroencephalogram was obtained from 4 children during functional hemispherotomy. IEDs/min were monitored using an automated epileptiform discharge detector. The changes in IEDs rates medians were assessed by a Wilcoxon rank sum test. Hemispherotomy always started with temporal-stem and temporo-mesial followed by corpus callosum and fronto-basal disconnection.

Results: Continuous recording of epileptic discharges was obtained during the entire surgery. The first patient (5y/o boy), IEDs/min were reduced after temporo-mesial disconnection $(\mathrm{p}<0.001)$, remained seizure free at one year (Fig1A). The second patient, (10y/o boy) had reduction in IEDs/min $(\mathrm{p}=0.01)$, was seizure free after surgery (Fig1B). The third patient, (6 m/o girl) showed no reduction in IEDs, required 2 further surgeries to become seizure free $(\mathrm{p}=0.2$, Fig1C). The last patient, ( $8 \mathrm{~m} / \mathrm{o}$ boy) showed marked reduction of IEDs $/ \mathrm{min}$ but with increased spike activity towards the end of surgery $(\mathrm{p}<0.001)$ (Fig1D), had post-operative epileptic irritative activity but was seizure free at one year follow-up.

Conclusion: We have shown that continuous intraoperative monitoring of IEDRs may provide an interesting tool to assess seizure outcome and completion of disconnection during hemispherotomy. It provides also information on epileptic discharges during surgery and can be used even in children less than one-year-old.

\section{4}

\section{Efficiency of CSF shunting in treatment for epilepsy}

Villiam A. Khachatryan, Alexander V. Kim, Konstantin A. Samochernykh Russian Polenov Neurosurgical Institute, Saint-Petersburg, Russia

Background: Epilepsy is relatively prevalent in children with hydrocephalus of various etiologies. There are many reports in which these issues have been extensively discussed, but their results are still diverse and they remain controversial in many aspects.

Material and Methods: Treatment of 128 epileptic patients, subjected to 155 primary (101 patient) and repeated (2-6) surgical procedures (27 patients) were analyzed. The dynamics of epileptic manifestations after hydrocephalus had been corrected was studied. Lumboperitoneostomy and ventriculoperitoneostomy were performed. Treatment results were evaluated through dynamics of clinical-electroencephalographic manifestations of epilepsy. MRI and CT findings, dynamic CSF studies were used to assess severity of hydrocephalus.

Results: Seizures were frequent (more than 1 per week, EEG findings of epileptic activity). All the patients suffered marked hydrocephalus. CSF hypertension was found $40.5 \%$, disturbed CSF resorption in $70.3 \%$ of the cases. CSF-shunting operations resulted in cessation of seizures in $53.6 \%$ of the patients. The better results were obtained in the group of generalized seizure cases, and in the patients with CSF hypertension or stable CSF resorption disturbances $(\mathrm{P}<0.05)$. In these cases shunt occlusion resulted in seizure recurrence, and restoration of CSF flow through the shunt - in less frequent seizures or absence of them.

Conclusion: The role of disturbed CSF circulation in pathogenesis of epilepsy and the problems of choosing treatment tactics for drug-resistant hydrocephalus-accompanied epilepsies are discussed.

\section{5}

\section{Experience of Peri-insular Anterior Quadrantectomy (PIAQ)} for intractable sub-hemispheric pediatric epilepsy

Kim Ju Seong ${ }^{1}$, Park Eun Kyung ${ }^{1}$, Yeom In Sun ${ }^{1}$, Bae Hyoun Je ${ }^{1}$, Kim Da Young ${ }^{1}$, Shim Kyu Won ${ }^{1}$, Kang Seok Gu ${ }^{1}$, Yang Kook Hee ${ }^{2}$, Park Seung $\mathrm{Woo}^{3}$, Kim Dong Seok ${ }^{1}$

${ }^{1}$ Department of Pediatric Neurosurgery, Severance Children's Hospital, Seoul, Korea; ${ }^{2}$ Department of Neurosurgery, National Health Insurance Service Ilsan Hospital, Koyang, Korea; ${ }^{3}$ Department of Neurosurgery, Kangwon National University Hospital, Chuncheon, Korea
Introduction: The operative techniques of peri-insular anterior quadrantectomy (PIAQ) was illustrated for patients with intractable subhemispheric pediatric epilepsy.

Methods: Two patients underwent peri-insular anterior quadranatectomy at Severance Children's Hospital. One patient was 11-year-old male. First brief tonic seizure occurred at 10-month-old and put on AED medication. However, seizure was not controlled for years. He tried vagus nerve stimulation and Ketogenic diet at another tertiary hospital but efforts were not effective. His epileptogenic focus was suspected right frontotemporal area on intensive presurgical evaluation. The other patient was 6-monthyear old girl without any perinatal complication. She started neonatal seizures of eyeball deviation and eye blinking since the $2^{\text {nd }}$ day of her life. She was diagnosed with infantile spasm and started on AED. In our presurgical evaluation, suspicious cortical dysplasia on left hemisphere and epileptogenic focus was suspected in left frontotemporoparietal areas. The anterior quadrantectomy was modified form of functional hemispherotomy with the goals of isolating the epileptogenic focus by disconnecting frontal and temporal areas.

Results: Despite of short-term post-operative follow-up periods, one patient achieved dramatic seizure control. However, the other patient had ongoing intractable epilepsy. That patient was underwent additional operation (functional hemispherotomy) before the discharge. After the $2^{\text {nd }}$ operation, $\mathrm{He}$ was free of seizure. Both cases did not have any surgical complications. Conclusions: Under the intensive pre-surgical evaluation and definitive surgical margin has been prepared, peri-insular anterior quadrantectomy is an effective disconnection surgery for sub-hemispheric pediatric epilepsy patients.

\section{6}

Experience of vagus nerve stimulation for medically refractory epilepsy in children and adolescents

Takamichi Yamamoto, Ayataka Fujimoto, Hirokazu Nakatogawa, Kyo Togasawa, Chikanori Inenaga, Tokutaro Tanaka

Department of Neurosurgery, Comprehensive Epilepsy Center Seirei Hamamatsu General Hospital, Hamamatsu, Japan

Rationale: Vagus nerve stimulation (VNS) was finally approved in 2010 by the Japan Ministry of Health, Labor and Welfare. VNS has been used worldwide for the treatment of medically refractory epilepsy if cranial surgery such as focus resection is not justified. There are no restrictions in terms of age and epilepsy syndromes in Japan.

Methods: Twenty patients under the age of 18 years old ( 3 - $18 \mathrm{y}-\mathrm{o}$, mean age $12.7 \mathrm{y}-\mathrm{o})$ underwent implantation of the VNS Therapy System from the official approval through December 2012 in our hospital. Indications for VNS were multifocal epilepsy (7 patients), generalized epilepsy (4), failed cranial surgery (8), and other reasons (1).

Results: Two patients $(10 \%)$ became free from seizures. Six patients $(30 \%)$ of them showed more than $50 \%$ seizure reduction. Less than $50 \%$ seizure reduction was seen in 8 patients $(40 \%)$, and no change in terms of seizure frequency was 4 patients $(20 \%)$. All patients well tolerated this treatment and none of them asked discontinuance even though patients were children or adolescents. Patients did not get any serious complications and surgical site infections in this series.

Conclusions: VNS is safe as previously reported, and demonstrated the same effectiveness for pediatric and adolescent patients with medically refractory epilepsy. We believe more patients in Japan should be treated by VNS if they are not good candidates for cranial surgery.

\section{Functional}

47

Impact of neurosurgical intervention for severe spasticity (ITB \& SDR) on spasticity related pain

Liana Beni-Adani ${ }^{1}$, Ron Lamdan ${ }^{2}$, Yael Harel ${ }^{1}$, Nava Gelkopp ${ }^{3}$, Aviva Fatal $^{1}$, Liora Sagie*, Hila Ben-Pazi ${ }^{3}$ 
Dana Children's Hospital ${ }^{1}$ (TASMC) and Tel-Aviv University, Hadassah Hospital and Hebrew University ${ }^{2}$, and Sharee Zedek Hospital ${ }^{3}$, Jerusalem

Introduction: Patients who are considered for a neurosurgical procedure to reduce spasticity (ITB Pump/SDR) may be divided to 2 Main Groups: a. Severely impaired individuals, "Low Function" (GMFCS V), in whom the goals of surgery would be to provide comfort, improve positioning and ease of care; b. "High Function" individuals, in whom the goals of treatment would be improving function. Patients from both groups may suffer from spasticity related pain; therefore decrease in pain would be an important step to improve quality of life.

Patients and methods: In a prospective series of spastic patients with GMFCS 2-5, 10 patients underwent Selective Dorsal Rhyzotomy (SDR) and 40 ITB pump implantation for severe spasticity. We looked at different clinical parameters including pain and discomfort before and after neurosurgical intervention.

Results: Pain/discomfort either during active/passive movements, or at rest (related to spasms) were important complaints (of patients or caregivers) in $84 \%$ patients $(42 / 50)$. In $44 \%$ pain and discomfort were the major complaint. In $38 / 42$ patients $(90 \%)$ ) there was significant improvement in pain and patients or caregivers reported facilitation of transfers, handling or physiotherapy due to decrease in pain during these activities. Conclusion: Decrease in pain was an achievable goal in most of the "low function" patients facilitating improved sitting, handling and transfers. In high function patients decrease in spasms or spasticity related pain enabled more intensive physiotherapy and exercise to maintain range of motion. This parameter in itself affected positively the quality of life in $90 \%$ of operated patients.

\section{8}

Electrophysiological monitoring applied to pediatric neurosurgery. A prospective study of 34 consecutive cases

José Aloysio CostaVal, Leopoldo Mandic Furtado, Etelvino Coelho Mota Pediatric Neurosurgery, Biocor Instituto, Vila da Serra Hospital, Nova Lima, Brazil

Introduction: The aim of this paper was to analyze the usefulness of electrophysiological monitoring in pediatric neurosurgery procedures in a tertiary care hospital.

Methods: In this study we analyzed 34 neurosurgical procedures performed with the aid of electrophysiological monitoring in 34 consecutive patients from January 2011 to December 2012. We considered the following variables: patients' age and gender, diagnosis, type of procedure, type of electrophysiological monitoring, intraoperative monitoring usefulness, surgery duration, mean hospital stay time and mortality rate. The electrophysiological monitoring was performed by the same neurologist.

Results: Most patients were girls: 15 (44\%). The surgical procedures performed were: anchored spinal cord surgery: 14 cases $(42.1 \%)$; infratentorial tumors: 7 cases $(20.5 \%)$; supratentorial tumors: 3 cases $(9 \%)$; optical nerve tumor: 1 case $(3 \%)$; optic nerve decompression for fibrous bone dysplasia: 01 case (3\%); selective dorsal rhizotomy: 5 cases $(14.7 \%)$; spinal cord tumors: $2(6 \%)$; syringomyelia: 1 case $(3 \%)$. Monitoring proved to be useful in $90 \%$ of the procedures, and it did not influence the surgical approach in $3 \%$ of the cases. We found a monitoring-related morbidity rate of $3 \%$. There was no relationship between the age and accuracy and safety.

Conclusion: Electrophysiological monitoring was useful in most pediatric neurosurgery procedures and seems to safe to patient.

\section{9}

Results of selective motor fasciculotomy in spastic upper limbs due to cerebral palsy

Anirudh K. Purohit ${ }^{1}$, Aneel K. Puligopu ${ }^{1}$, Srikanth S. Reddy ${ }^{1}$, Naveen K. Balne $^{2}$
${ }^{1}$ Department of Neurosurgery, ${ }^{2}$ Department of Physiotherapy, Nizams Institute of Medical Sciences, Hyderabad, India

Background/Objectives: To assess the outcome of selective motor fasciculotomy (SMF) in relieving upper limb harmful resistant spasticity and thereby to improve motor functions in persons with cerebral palsy. Design: Randomized Clinical Trial.

Participants and Setting: Thirty people having cerebral palsy with mean of age 12.66 years with upper-limb resistant spasticity.

Materials/Methods: The participants had spastic hemiplegia, triplegia and quadriplegia ( $\mathrm{n}=11,9,10$ respectively), were assessed using Modified Ashworth Scale, Selective Voluntary Control Grade (SVC), Wee FIM Scale and hand function evaluation. SMF was performed on musculocutaneous nerve $(n=15)$, median nerve $(n=35)$ and ulnar nerve $(n=3)$ for elbow flexors, pronators $\&$ radial wrist flexors and ulnar wrist flexors spasticity respectively. Pre and post op therapeutic exercises were performed.

Procedure: Under general anesthesia without muscle relaxants respective nerves were exposed. The component fascicles of the respective nerve branches were dissected, stimulated with bipolar current to identify the muscle and to observe the hyperactive response. Approximately $1 / 3^{\text {rd }}$ to $2 / 3^{\text {rd }}$ fascicles were ablated.

Results: Statistical analysis using Wilcoxon Signed Ranks test showed significant reduction in spasticity (p-0.0031) and; improvement in SVC ( $\mathrm{p}-0.0019)$, hand functions (grasp to hold a rod) and Wee FIM self care domain in 10.26 (range 4 to 30 ) months. There was no recurrence in spasticity and complication following surgery.

Conclusions: The SMF of musculocutaneous, median and ulnar nerves significantly reduces spasticity in the affected muscle groups and thereby improves the self-care (motor) functions in selected people with cerebral palsy who have harmful resistant spasticity. The spasticity does not recur and procedure is safe.

\section{0}

White matter repair and functional recovery after intra-arterial delivery of human NPC in rodent neonatal hypoxia-ischemia

Tenille Smith ${ }^{2}$, Laurie Chicha ${ }^{1}$, Sarah Rosenblum ${ }^{2}$, Raphael Guzman ${ }^{1,2}$ ${ }^{1}$ Neurochirurgische Klinik \& Brain Ischemia and Regeneration Group, Universitätsspital Basel; ${ }^{2}$ Department of Neurosurgery, Stanford University, School of Medicine, Stanford, United States

*Presenter

Aims: White matter damages in the immature brain are a hallmark of neonatal hypoxia-ischemia (HI) and cerebral palsy, often causing lifepersisting sensory-motor and cognitive deficits in the growing child. Preclinical stem cell transplantation has demonstrated endogenous repair mechanisms including neurogenesis. However the specific impact on myelination in the developing brain has not been adequately characterized. Using a neonatal rat $\mathrm{HI}$ model, we investigated the effect of human neural progenitor cell (NPC) transplantation on myelination and functional recovery.

Methods: Rats underwent common carotid artery ligation followed by hypoxia on postnatal day 7 (P7). At P10, luciferase-expressing NPCs were intraarterially injected in immunosuppressed neonates, allowing their monitoring via in vivo bioluminescence. NPC survival and differentiation as well as endogenous myelination were monitored by immunohistochemistry at 10/30 days post-treatment. RT-qPCR was performed on stroked hemispheres in NPC- and saline-treated groups. Functional recovery was assessed at $\mathrm{P} 40$.

Results: BLI and histology demonstrated homing of NPC to the stroked hemisphere at 3/10/30 days after treatment. Myelin-protein staining demonstrated enhanced myelination in the corpus callosum and the striatum at 10/30 days after celltreatment. Increased expression of transcripts specific for myelination, cell proliferation and immunomodulation were observed in the stroked hemisphere of celltreated animals. Neonates transplanted 
with NPC demonstrated better performance on elevated plus maze (EPM) and novel object recognition (NOR).

Conclusions: Although stem cell therapy is already accepted as a promising therapeutic approach for children with $\mathrm{HI}$ injuries, we here suggest one potential mechanism of action is the functional repair of white matter tracts.

\section{1}

Midline cavum: The normal, the abnormal and the pathological - A neuropsychological link to the enigmatic continuum

Suhas Udayakumaran ${ }^{1}$, Sandhya Cherkil ${ }^{2}$

${ }^{1}$ Division of Pediatric Neurosurgery, ${ }^{2}$ Department of Clinical Psychology, Amrita Institute of Medical Sciences and Research Centre, Kochi, Kerala

Background: Cavum septum pellucidum (CSP) and Cavum Vergae (CV), formerly called the fifth and sixth ventricles of the brain are poorly understood midline structure.

Case report: A 13-year-old boy presented to us with episodic headache, vomiting and imbalance of around 1 week duration. On examination he had bilateral cerebellar signs. MRI showed bulging cavum with CISS sequence showing foramen of Monro obstruction. Assuming intermittent foramen of Monro obstruction to be the cause, he underwent endoscopic fenestration of the septum. He was relieved of his symptomatology immediately after the surgery. At 6 months follow-up, the symptoms have improved completely with corroboratory imaging findings. His immediate preoperative status could be explained by the imaging findings. However interestingly enough, his preoperative clinical psychology assessment revealed severe cognitive disturbances of executive functions, memory, attention, and visuospatial perception which were not a part of the presenting complaints. Behaviorally it was reported that he lacked motivation to socialize, and did not take effort in cognitively demanding tasks. Postoperatively he showed marginal improvement in cognitive findings, in contrast to the dramatic improvement in the preoperative presenting symptoms Discussion: In this article the authors try to unravel the entity of midline cavum and its relevance in normal subjects (20-25\%), behavioral issues, psychiatric illness and as a structural abnormality.

Conclusion: The cavum and its role in normal subjects, contribution to psychological abnormalities and pathological presentation remains a grey zone but it is clear that the presentation represent an enigmatic continuum with varying cognitive and behavioral involvement.

\section{2}

\section{Abnormal flexion reflex in lesions of the pyramidal tract}

Galimzhan M. Yelikbayev, K. Bralov, Y. Annaoravoz

Department of the General Surgery International Kazakh-Turkish University, Turkistan, Kazakhstan

We have developed a new method for diagnosing lesions of pyramidal tract, causing jog pathological flexion reflex. In the process of diagnosis of lesions of pyramidal tract, which includes flexion exercise on pathological reflexes, quick irritation on the plantar surface of the foot, starting at the base of the toes to the heel, and that causes a bending of the fingers and nodding 2-5 nail bone. The effectiveness of the method is the wide scope of reflex zones (over the entire surface of the plantar side), and a stable, bringing about a distinct flexion (bending) of the toes at 1-5 pyramidal insufficiency (abnormal reflex). When we developed a new method of determining the plantar flexor reflex irritation covers both the reflex zones 3-4 cutaneous peripheral nerves (n. Plantaris medialis et lateralis, from branches $n$. Tibialis, rr. Cutaneus cruris medialis et $n$. saphenus and N.Cutaneus dorsalis et lateralis). Chafe receptors and impulses in their respective segments of sensory - motor neurons in the spinal cord and are responsible stereotypical in all cases, in the absence of the brake controller CNS. Irritation of a nerve in each individual signature of each time extensor effect does not. that confirms the importance of the overall reaction. Method developed and was many clinical examinations in the neurosurgery department. Clinical data proved instrumental diagnostic methods (CT, MRT).

\section{Hydrocephalus}

\section{3}

Long-term follow-up in children with VP shunt

Fernanda Andrade, Hamilton Matushita, Daniel D. Cardeal, Manoel Teixeira

Hospital das Clinicas, University of Sao Paulo, Sao Paulo, Brazil

Introduction: The placement of VP shunts is the most common procedure in pediatric neurosusrgery. Preoperative and postoperative care, standard techniques to VP shunt insertion are essential to avoid major complications as infection and mechanical malfunction. Patients without or few system revision may have long survival. About 1500 patients are currently followed at Hospital das Clinicas, pediatric neurosurgery section. Forty-five percent of them are patients with hydrocephalus and around $55 \%$ of surgical procedures are related to hydrocephalus.

Methods: Thirty-eight patients with hydrocephalus and long-term followup (more than 18 years) are selected. Data collected were the cause of hydrocephalus, age at first surgery, number of revision.

Results: Fourteen patients underwent only one VP shunt placement and nine required only one shunt revision, usually due to mechanical malfunction. Seven patients need more than 2 procedures to shunt revision, most of them are related with shunt infection. Twenty patients had congenital malformations and 13 patients had association with spinal or cranial dysraphim. Congenital malformations need shunt revision in 14 cases and all three patients with cranial dysraphism had more than one shunt procedure. Only one patient with spinal dysraphism need 3 procedures due to shunt infection; other 2 patients had mechanical malfunction and need one more procedure; 7 patients had no shunt revison. Age at first procedure was not related with shunt revision.

Conclusion: Long survival for patients with hydrocephalus is possible and was related with no or few shunt revisions in our revision.

\section{4}

Forgotten hydrocephalus valves

Alfred Aschoff

Neurochirurgische Universitätsklinik Heidelberg

Introduction: 290 hydrocephalus valves have been concepted, designed, patented or produced since 1949; about 160 are available on the marked in 2013. Where are the 130 missing designs?

Methods: We analyzed 530 articles related to the history of hydrocephalus, all available catalogues of shunt-producers inclusively of old editions in the seventies, $>60$ patents, and personal communications of Pudenz, Holter, S \& C.Hakim, Schulte, Portnoy, Scott, Shillito, Raimondi, Voth, Schubert, Kuffer, Grotenhuis, Paes, Hokanson, Nissels, Serlo, Chhabra, Miethke and many coworkers of shunt companies.

Results: The ball-valve of Nulsen and Spitz was often published, the same is true for the classical designs of Holter and Pudenz/Schulte. In contrast, the early adjustable designs of V.Bush and Kuffer/Strub, the gravitational valves of Holter, Portnoy and Sainte-Rose, the variable resistance valve of MBB/Hildebrand, the CSF-pump of Mathews, the distal slit valve of Ames, the Indian flap-valve of Upadhyaya, are forgotten or citated by a few experts only. We present examples of either interesting designs or typical clones.

Conclusion: 1. Rare valves may confuse neurosurgeons in revisions. 2. The time lag between first concepts, serial production and general acceptance can reach 60 years. 


\section{5}

Neuronavigation in pediatric neurosurgery - indications and use of the Axiem magnetic navigation system

David Breuskin, Christoph Tschan, Joachim Oertel

Department of Neurosurgery, Saarland University, Homburg/Saar, Germany

Introduction: Intraoperative safety and certainty of localisation is essential to any surgical procedure. In certain cases of pediatric neurosurgery, we used magnetic navigational systems or standard navigation to optimise trajectory planning for minimally invasive surgery.

Materials and methods: From January 2011 until April 2013, 61 children (mean age 10,4 y) underwent navigation-assisted surgical procedures. 21 were treated using the Axiem magnetic navigation. Tumour resection accounted for $43 \%, 16 \%$ for etV, $7 \%$ arachnoid cysts, $15 \%$ for cystostomy, $10 \%$ for vascular malformations and $9 \%$ for difficult shunt placements including aqueductoplasty. Indications for the magnetic navigation were procedures in newborns where sharp fixation was unfavourable, cases where intraoperative movement of the head was required as well as placement of catheters in the fourth ventricle. Intraoperative screenshots were compared to postoperative neuroradiologic imaging.

Results: In all cases, preoparative planning could be implemented and led to fast and secure surgical procedures. Precision was equal to both systems and proved effective and successful when compared to postoperative imaging. The magnetic system allowed navigation through metallic endoscopic work sheets. In case of head movement, it remained precise. Flexible navigation within catheters allows exact placement of the tip in the ventricular system, especially in the fourth ventricle during aqueductoplasty.

Conclusion: Magnetic navigation proved to be as precise as standard navigation. Safety was improved and supported minimally invasive treatments. Though its costs make it more expensive than standard systems, the benefits make it essential for certain indications and widens the spectrum of neuronavigation in pediatric neurosurgery.

56

Pediatric neuroendoscopy: A review of seventeen years experience José Aloysio CostaVal, Leopoldo Mandic Furtado

Pediatric Neurosurgery, Vila da Serra Hospital, Nova Lima, Brazil

Introduction: The objective of the study was to analyze the neuroendoscopy experience in the pediatric population of a neurosurgical tertiary center.

Material and methods: A retrospective study analyzed the neuroendoscopic procedures performed in 400 patients between January of 1997 and December of 2012. The variables analyzed were gender, hydrocephalus etiology, associated comorbidities, type of endoscopic procedure performed, intraoperative time, length of hospital stay and mortality/morbidity rates. All patients were 18 years old or younger.

Results: A total of 600 neuroendoscopic procedures were performed during this period with $422(70.3 \%)$ in the pediatric population. In this final cohort, there was a male predominance $(61 \%)$. Endoscopic third ventriculostomy (ETV) was the most common procedure (77.3\%) followed by septostomy (21.6\%) with an overall mortality rate of $1.25 \%$ Obstructive hydrocephalus was observed in $79.8 \%$ of the cases, aqueduct stenosis in $36.13 \%$, tumoral lesions in $22.2 \%$ and intraventricular hemorrhage in $14.8 \%$.

Conclusion: This study shows the evolution of a tertiary pediatric neurosurgery service in neuroendoscopic treatment of hydrocephalus, corroborated by its increasing variety and complexity of cases and with a low mortality rate.
57

Neuroendoscopy applied magnetic neuronavigation in the treatment of complex hydrocephalus and intracranial cysts in a prospective study with 14 patients

José Aloysio CostaVal, Leopoldo Mandic Furtado

Pediatric Neurosurgery, Vila da Serra Hospital, Nova Lima, Brazil

Introduction: conventional endoscopic navigation in complex hydrocephalus is very difficult due to distortion of the normal anatomy and the neurosurgeon's difficulty in recognizing the structures. Magnetic neuronavigation could be a valuable tool helping the procedure.

Methods: In this prospective study of patients operated from January 2011 to December 2012 we analyzed all complex hydrocephalus and intracranial cysts operated with neuronavigation coupled with neuroendoscopy. We evaluated the following variables: patient distribution in age, gender, hydrocephalus etiology, type of endoscopic procedure employed, morbidity rate, surgery duration and mean hospital stay. We took off this study all those patients submitted to conventional neuroendoscopy.

Results: We performed 21 surgical procedures in 14 patients. They had the same gender distribution and mean age of 7 years. The isolated fourth ventricle was observed in 2 patients (14.3\%); complex hydrocephalus in 8 cases (57\%); Dandy Walker syndrome in 2 cases (14.3\%) and arachnoid cyst in 2 cases $(14.3 \%)$. Brain shift impacted the efficacy in a procedure $(0.2 \%)$. There was no mortality.

Conclusion: Magnetic neuronavigation associated with neuroendoscopy seems increased the efficacy and safety of endoscopic procedures in the population studied.

\section{8}

\section{Osteopetrosis patients during pediatric neurosurgery practice}

Mostafa El Khashab

Hackensack University Medical center, New Jersey, United States

Osteopetrosisa is characterized by osteoclastic malfunction that results in increased bone density and consequences such as bone marrow insufficiency, bone fragility and neurological symptoms due to increased skull thickness. The disease has some known neurological manifestatiwas that brings the child to neurosurgery clinics. Here we report our esperience with this disease and describe some patients whose disease was discovered firstly with neurological presentations. Intracranial hypertension symptoms were the first presentation of osteopetrosis in several children that managed with shunt procedure or conservative treatment; most had signs and symptoms of hydrocephalous and some had pseudotumor cerebri. Visual disturbance was a common finding. The common cause of death in our patients were medical complications of this disease including sepsis. Discovering osteopetrosis with neurological mnaifestations is very rare and mostly neurological complications are found during follow-up of child with osteopetrosis. Extensive investigation of a child with new onset hydrocephalus is mandatory to prevent more serious complications of underlying disease.

\section{9}

Indications for valve-pressure adjustments of gravitational assisted valves in patients with idiopathic normal pressure hydrocephalus Leonie Gölz, Johannes Lemcke, Ullrich Meier

Department of Neurosurgery, Unfallkrankenhaus Berlin, Germany

Background: Modern ventriculoperitoneal shunts have programmable valves to adjust valve-pressure according to the patients' individual needs. The aim of this retrospective analysis is to evaluate indications for valvepressure adjustments in Idiopathic Normal Pressure Hyrocephalus (iNPH).

Methods: Patients operated between 2004-2011 diagnosed with iNPH were included. Kiefer-Scale was used to classify each patient. 
Follow-up exams were conducted three, six, and 12 months after shuntimplantation and yearly thereafter. Initial valve-pressure was 100 or $70 \mathrm{mmH}_{2} \mathrm{O}$. Planned and reactive adjustments of the valvepressure were carried to optimize therapy and to avoid over- and underdrainage.

Results: 180 reductions of the valve-pressure took place. Most patients $(89 \%)$ needed one or two adjustments of their valve-pressures for optimal results. In $41 \%$ an improvement of symptoms was observed. Gait disorder was improved most often after valvepressure adjustments (32\%). 18 times an elevation of valve-pressure was necessary because of headaches, vertigo or the development of subdural hygroma. Optimal valve-pressure for most patients was around $50 \mathrm{mmH}_{2} \mathrm{O}(36 \%)$.

Conclusion: Shunt therapy in iNPH should aim at valve-pressure settings between $30-70 \mathrm{mmH}_{2} \mathrm{O}$. Reactive adjustments of the valve-pressure are useful for therapy of over- and underdrainage symptoms. Planned reductions of the valve opening pressure are effective even if postoperative results are already satisfactory.

\section{0}

\section{Electromagnetic navigated endoscopy in children in the first year} of life

Elvis J. Hermann, Florence Choumin Nguemaleu, Yvonne Mondorf, Joachim K. Krauss

Department of Neurosurgery, Medical School Hannover, Hannover, Germany

Background: In navigated endoscopy usually sharp head fixation is needed. In children younger than 1 year of age sharp head fixation is associated with the risk for skull fracture and resulting complications.

Objective: Here, we demonstrate the advantage of the use of an electromagnetic navigation system in children younger than 1 year of age for endoscopic procedures obviating the need of sharp head fixation.

Methods: Five children ( 4 boys, 1 girl) between 12 days and 11 months (mean age: 5,9 months) underwent electromagnetic navigated endoscopy for treatment of cystic cerebral lesions based on MR imaging. The patients were registered for navigation in supine position and after confirming accuracy they were repositioned for endoscopic surgery. The head was lightly fixed by a tape to the horseshoe-headholder without rigid head fixation. Navigation was performed after virtual planning using a stylet introduced into the endoscope.

Results: In all 5 patients electromagnetic navigation-guided endoscopy was successful without complications due to the navigation system. Navigation accuracy was between 2 and $4 \mathrm{~mm}$. The electromagnetic navigated approach allowed to achieve the goal of surgery in all five patients and to realize the planned fenestration of the cystic lesion. It was considered an easy-to-use and very helpful technique in all procedures.

Conclusion: Electromagnetic navigated endoscopy in children under 1 year of age is a safe method enabling endoscopic treatment of complex cystic formations without the need for sharp head fixation.

\section{1}

Neurocutaneous melanosis associated with hydrocephalus and syringomyelia - Case report and literature review

Wing-Mann Ho, Claudius Thomé, Ilse Laimer

Department of Neurosurgery, University Hospital Innsbruck, Austria

Neurocutaneous melanosis ( $\mathrm{NCM})$ is a rare noninherited embryonal neuroectodermal dysplasia characterized by increased proliferation of melanin-producing cells in the skin, brain and leptomeninges. Neonates with NCM may develop hydrocephalus, seizures, cranial nerve dysfunction and involvement of the spinal cord and roots. Once there are neurological symptoms, the prognosis is poor. We present the case of a male neonate born with multiple congenital melanocytic naevi. Within one year he developed an arachnoid cyst in the posterior fossa with consecutive hydrocephalus and a ventriculoperitoneal Shunt was placed. Three years later the patient demonstrated progressive paralysis and MRI revealed a multi-septated holocordal syringomyelia. Suboccipital decompression with $\mathrm{C} 1$ laminectomy was performed. Imaging showed enhancing thickened leptomeninges, which appeared as strongly pigmented membranes intraoperatively. Histopathological analysis confirmed a leptomeningeal melanosis. Due to later progression of paralysis, intermittent breathing difficulties and increasing syringomyelia, revision surgery with prolongation of laminectomy, adhesiolysis and partial exstirpation of the cerebellar tonsils was performed. A few months later the child died of respiratory failure. About 100 cases of NCM have been reported in total. The prognosis of symptomatic NCM is very poor with progressive deterioration and early death. Chemotherapy and radiotherapy were shown to have only little effect on the rapid course of NCM, so that there currently are only palliative treatment options. Hydrocephalus, syringomyelia and lesions in the brain tissue were described in 2 cases in the literature. In our patient only the leptomeninges were affected. Nevertheless, CSF drainage was severly impaired, which could not be effectively corrected surgically.

\section{2}

Endoscopic fenestration of pediatric intraventricular arachnoid cysts: Report of 2 children

Yusuf Izci, Ozkan Tehli, Ilker Solmaz

Department of Neurosurgery, Gulhane Military Medical Academy, Ankara, Turkey

Intraventricular arachnoid cysts cause macrocephaly and neurological disturbances in children. The traditional treatment is cystoperitoneal shunting, but recently endoscopic techniques are in use for the definitive treatment of this rare disorder. The aim of this report is to present our experience on the endoscopic management of intraventricular arachnoid cyst in 2 children. First patient is a 2,5-year-old male child who previously underwent ventriculoperitoneal shunting for the treatment of hydrocephalus and left lateral ventricle arachnoid cyst. The shunt is failed and the cyst gradually enlarged with worsening clinical condition of the patient. He underwent endoscopic fenestration of the arachnoid cyst from the upper and lower parts of the cyst. He improved after surgery. Second patient is a 3-year-old male child who previously underwent shunting for the treatment of left lateral ventricle giant arachnoid cyst. The shunt is failed and the cyst continued to enlarge. Right hemiparesis occurred during this period and he underwent endoscopic fenestration of the arachnoid cyst from the lateral side of the cyst. His hemiparesis improved after endoscopic surgery. Endoscopic fenestration is a treatment option for intraventricular arachnoid cysts in children. This technique should be performed by the experienced surgeons and the patients should be followed-up with short intervals in order to assess the success of the fenestration.

\section{3}

\section{Gravitational valves - higher opening pressure results in less} ventricle collapse

Beatrice Jager, Jürgen Krauss, Hartmut Collmann, Tilmann Schweitzer Section of Pediatric Neurosurgery, Department of Neurosurgery, University of Würzburg, Würzburg, Germany

Introduction: There is an ongoing discussion on shunt systems and pressure levels in hydrocephalus therapy. In the very young usually a 
low pressure level is chosen. Since 1998 we are using gravitational valves (Aesculap, Miethke GAV) with an opening pressure of 10/40 cm. For treatment of slit ventricle syndrome or pseudotumor cerebri we chose an opening pressure of $15 / 50 \mathrm{~cm}$.

Methods: We report on 193 consecutive cases of implanted GAV 10/40 and 41 cases of GAV 15/50 from 1998 to 2012.

Results: We saw 4 cases $(2.1 \%)$ of bilateral ventricle collapse and 10 cases $(5.2 \%)$ of unilateral ventricle collapse using GAV 10/40. In GAV $15 / 50$ valves we had no bilateral ventricle collapse and 5 $(12.2 \%)$ children with unilateral ventricle collapse. Reoperation rate for valve failure was similar in both groups of GAV 10/40 and GAV $15 / 50$ (18,7 \% vs. 14,6 \%). Downgrading of the valve was necessary in 8 cases $(4,1 \%)$ for GAV $10 / 40$ and 2 times (4,9\%) for GAV $15 / 50$ valves

Conclusion: In the very young with hydrocephalus valves with higher opening pressure levels offer effective treatment while preventing the development of collapsed ventricles and its possible deleterious side effects. We therefore believe that valves like the GAV 10/40 and GAV 15/50 are a good choice in treatment of pediatric hydrocephalus.

\section{4}

Ureaplasma urealyticum - an undetected enemy in posthemorrhagic hydrocephalus of preterms

Elke Januschek $^{1}$, Judith Jochim ${ }^{2}$, Nader Gordjani ${ }^{2}$, Peter T. Ulrich ${ }^{1}$

${ }^{1}$ Department of Neurosurgery, ${ }^{2}$ Department for child and adolescent medicine, Klinikum Offenbach GmbH, Offenbach, Germany

Introduction: We report a case of a preterm with posthemorrhagic hydrocephalus $(\mathrm{PH})$ and prolonged high protein count $(\mathrm{PC})$ in the cerebrospinal fluid (CSF) without germ-proof in routine lab tests. At last in pcr test ureaplasma urealyticum were found as the cause of infection.

Case report: S. was born as a premature third triplet at 24 weeks of gestation $(605 \mathrm{~g})$ and developed an intraventricular hemorrhage on day 4. Several days later a dilatation of the ventricles was observed and an external ventricular drainage (EVD) was implanted. The initial cell number was 779 with a PC of $4773 \mathrm{mg} / 1$. Repeated replacements of the ventricular drainage and reservoir under antibiotic therapy could not normalize the protein, lactat and glucose counts, nor was a germ isolated. The PC increased to a maximum value of $14.474 \mathrm{mg} / \mathrm{l}$. After expanding the liquor studies ureaplasma urealyticum was finally detected. Chloramphenicol was given in a dosage of $700 \mathrm{mg} / \mathrm{kg}$ over 7 days, after the preterm failed to respond to doxycycline. Only after switching the antibiotic eradication succeeded. A change of the unishunt was not required. Later, a valve was interposed. Overall it was a highly complex case history with many accompanying diseases, epilepsy and psychomotor retardation.

Conclusion: In case of PH in preterms with sustained high PC, without germ-proof in routine lab tests and only mild signs of meningitis the investigation of the CSF for ureaplasma urealyticum is recommended. In our case the therapy with doxycyclin failed, the treatment with chloramphenicol was successful. The explantation of the shunt system was not required.

\section{5}

Pitfalls in the surgical treatment for posthemorrhagic hydrocephalus in premature infants: an experience report

Gertrud Kammler ${ }^{1}$, Pedram Emami ${ }^{1}$, Susanne Schmidtke ${ }^{2}$, Manfred Westphal ${ }^{1}$

${ }^{1}$ Department of Neurosurgery, ${ }^{2}$ Department of Pediatrics, Section Neonatology, University Medical Center Hamburg-Eppendorf

Introduction: Premature infants with posthemorrhagic hydrocephalus bear a high risk of complications when treated with VP (ventriculo- peritoneal) shunt. The aim of this study was to assess surgical complications in this cohort.

Methods: From 2009 to 2013, 298 premature infants with VLBW (very low birth weight) and ELBW (extremely low birth weight) were born in our level I neonatology unit; 11 suffered from posthemorrhagic hydrocephalus Papile grade III or IV with ventricular dilatation and intracerebral bleeding. Their charts were reviewed concerning gestations age, birth weight, head circumference, time of surgical procedure and complications.

Results: The mean gestational age was 26 weeks, the mean birth weight was $870 \mathrm{~g}$. All patients underwent serial lumbar punctures preoperatively. Implantation of subcutaneous reservoir was performed in four patients, six were treated with a valveless catheter temporarily, 1 was additionally treated endoscopically, and 11 received a VP shunt. Two patients showed recurrent tense fontanelles after shunt implantation and underwent revision surgery. 1 sustained overdraining with intracranial bleeding, two had circumscribed wound dehiscence, caused by suture material and three suffered from shunt infection. All patients underwent ultrasound and 1 had an MRI.

Conclusions: The optimal timing of surgical treatment and the decision on the appropriate device and the procedure is always a challenge in premature infants and should be a carefully planned, case-by-case decision involving both the neurosurgeon and the neonatologist.

66

Hydrocephalus: a symptom, a syndrome, a disease

Villiam A. Khachatryan

Russian Polenov Neurosurgical Institute, Saint-Petersburg, Russia

Aim: To precise the existing terminology, classification and the concept of diagnose-making and treatment of hydrocephalus.

Material and methods: 3500 patients since 1980 to 2012, 2448 CSFshunting procedures, 646 Torkildsen's operations, 136 ventriculostomies, 68 glomectomies and ventriculosubgaleal anastomoses.

Results: At the initial stages of hydrocephalus the main pathogenetic role is played by the primary cause of the disease, with hypertensive syndrome dominating clinically; abolishing the cause or balancing CSF circulation, i.e. etiopathogenetic treatment, are effective. At the late stages the vicious circle is noted to form, the basis of which is enlargement and deformity of CSF pathways with ventriculomegaly, dural sinuses deformity and occlusion, lowered perfusion pressure, and decreased brain elasticity. This system may be responsible for further progress of hydrocephalus together with the suggested etiologic factor or without it. At the late stages hydrocephalus gets to become a disease. In 294 patients CSF space enlargement was vicarious and not participating in pathogenesis of the disease, denoting brain atrophy solely. These were the patients under 2 or over 60, and those post intoxication.

Conclusion: It is expedient to classify hydrocephalus as a symptom, not manifesting clinically and requiring no correction, hydrocephalus as a syndrome, with the etiologic factor taking an important pathogenetic role, intracranial hypertension prevailing clinically, and etiopathogenetic treatment required, and hydrocephalus as a disease, when a pathologic system is being formed, and to treat means to shunt CSF. One should recognize hydrocephalus before CSF-shunting procedures, and drainage-depending hydrocephalus.

\section{7}

Intra-abdominal huge pseudocyst following ventriculoperitoneal shunt procedure

Vaner Köksal $^{1}$, A. S. Calapoglu ${ }^{2}$, A. Kanat ${ }^{1}$

${ }^{1}$ Recep Tayyip Erdogan University School of Medicine, Department of Neurosurgery, Rize, Turkey; ${ }^{2}$ Recep Tayyip Erdogan University School of Medicine, Department of pediatric surgery, Rize, Turkey

Introduction: All neurosurgeons struggle with shunt malfunctions and their complications. It is well known that the placement of a ventriculoperitoneal shunt (VPS) is still amongst the most frequently 
performed operations in the management of hydrocephalus. We investigated the literature and found many cases with abdominal pseudocyst, however no case with such a huge cyst as in our case has not been reported. So we decided to present this unusual case.

Case report: A 6-year-old male case admitted with abdominal discomfort and distention without neurologic deficit. The patient was paraplegic and was operated for myelomeningocele after birth. The computed abdominal tomography revealed that whole abdominal organs were covered with huge cysts, and peritoneal catheter of the shunt was inside of cyst. Laparotomy performed jointly by neurosurgeons and pediatric surgeons, exposed the catheter and removed from the cyst. Front wall of the cyst were completely damaged and the peritoneal catheter of the shunt was placed to a different region. During the follow-ups, no abdominal distention formation was observed again.

Discussion: One of the reason of shunt dysfunction is the abdominal pseudocysts formation. It has been a rarely encountered complication in pediatric neurosurgical practice. Although there have been no specific evidences related to the intra-abdominal cerebrospinal pseudo-cyst development, the hypothesis of inflammation development against the catheter and BOS has been accepted. The adhesions caused by the sterile inflammation have resulted in cystic cavity formation which does not have a real wall.

\section{8}

A rare reason of the congenital communicating hydrocephalus Hurler Syndrome Vaner Köksal ${ }^{1}$, A. Șen ${ }^{2}$, B. Erdivanlı ${ }^{2}$

${ }^{1}$ Recep Tayyip Erdogan University School of Medicine, Department of Neurosurgery, Rize, Turkey; ${ }^{2}$ Recep Tayyip Erdogan University School of Medicine, Department of anesthesia, Rize, Turkey

Mucopolysaccharidosis (MPS) is a rarely-seen, autosomal recessive inherited, multi-systemic lysosomal storage disease emerging as result of the specific lysosomal enzyme deficiency that degrades the mucopolysaccharides (glycosaminoglycan). Clinical findings emerged as result of the sub-endothelial deposition of non-degraded glycosaminoglycan in different systems. Clinical findings such as abnormal facial appearance, big tongue, macrocephaly, hepatosplenomegaly, bone deformities, corneal blurring, and mental retardation occurred in MPS. Especially skeleton deformities contingent upon the skeleton dysplasia were prominent in the clinical table. Also angina pectoris attacks due to the thickness of coroner can be expected. Emergence of the clinical symptoms in those cases which have had a normal appearance on birth have taken 6-12 months and average lifetime has been generally presented as between 5-10 years due to the cardiopulmonary insufficiency. It has been specified in cases that cerebrospinal fluid absorption was damaged depending upon the metabolites depositing in pia-arachnoid dura. Therefore, a slowly progressive chronic hydrocephalus table has occurred. It is generally diagnosed before the 18th month in children. The case we presented were genetically researched when the macrocephalic appearance was noticed when he was 3-month old and MPS type 1 was diagnosed (Hurler syndrome). In subsequent long-standing follow-up, the case was observed as having a slow increase around the head. There was no medical intervention until the age of 8 . The female case who had appearance like a 2-3 year old child was implemented ventriculo-peritoneal shunt when the case was determined as having a radiologically specific hydrocephalus upon the headache and learning disability complaints.

69

Fracture and subsequent skin perforation of the peritoneal catheter of a VP-shunt in a young adult

Julia Masomi ${ }^{1}$, Sven Kantelhardt ${ }^{1}$, Wolfgang Wagner $^{2}$

${ }^{1}$ Department of Neurosurgery, ${ }^{2}$ Section of Pediatric Neurosurgery, University Medical Center Mainz, Germany
Complications related to the peritoneal catheter in VP-shunts are fracture, disconnection, migration, perforation of organs etc. We report a case of shunt fracture and subsequent skin perforation at the chest. A 24-year-old male dysrhaphic patient with VP-shunt presented with a wound at the chest with secretion of cerebrospinal fluid. X-ray revealed shunt fracture with a sharp catheter end perforating the skin. The perforating part of catheter (with intense calcifications) was removed, the shunt ligated and the skin closed. Six months later, the patient presented with a new shunt fracture, skin perforation and CSF fistula, several centimeters more proximal. He underwent a second revision; the catheter was ligated now at a retroauricular level, where there was no relevant shunt calcification. The clinical course thereafter was uneventful (follow-up 8 months). There are a few reports in the literature on intenstinal or bladder perforation of a peritoneal catheter with subsequent extrusion through the anus, the urethra or even the mouth. There is only one publication describing a skin perforation at the scrotum in a 45 days old infant, operated on 6 weeks before [Saeidi Borojeni et al. 2012]. In this case, however, the shunt was not fractured. There exists a known risk of fracture of a calcified peritoneal catheter in long standing VP-shunts. The perforation of the skin by the fractured catheter, however, seems to be extremely rare and, to be best of our knowledge, this is the first case reported in literature.

70

Long-term outcome of shunting for the treatment of arachnoid cysts in children under 2 years of age

Masato Nagasaka ${ }^{1,2}$, Mihoko Kato ${ }^{1,2}$, Hirokatsu Osawa ${ }^{1}$

${ }^{1}$ Department of Neurosurgery, Aichi Children's Health and Medical Center, ${ }^{2}$ Department of Neurosurgery, Central Hospital, Aichi Prefectural Colony

Introduction: The purpose of this study was to assess long-term outcome of children with arachnoid cyst(AC) who underwent shunting under 2 years of age.

Methods: Between 1984 and 2009, thirty patients (19 males and 11 females) with symptomatic AC underwent surgery at the authors' institution. The mean age of the first operation was 9.2 months. The median follow-up period was 10.9 years.

Results: Presentaion: macrocephaly (head circumference (HC $>+2 \mathrm{SD})$ in 14 , increasing $\mathrm{HC}$ in 13 , cyst enlargement in 8 , developmental delay in 11 and so on. Locstion: six cysts $(20 \%)$ in sylvian fissure, six in interhemispheric fissure, six in intra- or paraventricle, six in infratentorial, three at suprasellar, one at quadrigeminal and two cysts spreading supraand infratentorial. Twenty children $(67 \%)$ received shunting (17 cystoperitoneal shunt, 3 ventriculoperitoneal shunt, 3 of 20 combined with fenestration) as first-line procedures and remainig 10 patients were operated on with craniotomy or endoscopic fenestration without shunting. Eight out of 10 without shunting were forced to be inserted shunt later. After all 28 patients needed shunting for the treatment of cysts, hydrocephalus, and subdural fluid collection. Nine out of 20 with shunting had not received additional surgery during follow-up periods (mean; 10.5 years). Follow-up image demonstrated almost cyst disappearance in 18. Intellectual status was normal in 16, borderline in 5, retardation in 9.

Conclusion: In terms of long-term outcome, cystoperitoneal shunt is a safe and effective procedures for ACs in the children under 2 years of age.

\section{1}

Treatment results and outcomes of obstructive hydrocephalus associated with unidentified bright object in neurofibromatosis type 1

Tatsuki Oyoshi, Hirofumi Hirano, Shingo Fujio, Hiroyuki Uchida, Manoj Bohara, Hiroshi Tokimura, Kazunori Arita

Department of Neurosurgery, Graduate School of Medical and Dental Sciences, Kagoshima University, Kagoshima, Japan 
Introduction: Treatment outcomes of obstructive hydrocephalus $(\mathrm{OH})$ with unidentified bright object (UBO) in neurofibromatosis type 1 (NF1) are still unknown. We reviewed the treatment outcomes in children with the coexistence of $\mathrm{OH}$ and UBO in NF-1 who underwent ventriculoperitoneal shunt (VPS) or endoscopic third ventriculostomy (ETV) over the last decade.

Methods: The patients with the coexistence of OH and UBO in NF-1 treated and followed at Kagoshima University Hospital between 2000 and 2012 were reviewed. All the patients were examined with a 1.5T MR imaging and focal areas of high signal intensity on T2-weighted images were confirmed.

Results: Four patients were diagnosed as OH with UBO in NF-1. The mean age at surgery was 15.2 (range 9-26) years. ETV was performed in 2 patients and VPS in 2 patients. MR images of all the patients demonstrated $\mathrm{OH}$ caused by aqueductal stenosis and UBO in the basal ganglia, cerebellum, pontine tegmentum and periaqueductal region. Preoperative symptoms were headache, convulsion and abnormal behavior. One patient was followed using MR imaging 7 years before the onset of hydrocephalus symptoms, which confirmed normal ventricle and aqueduct without $\mathrm{OH}$. Due to enlarged lateral ventricle with narrow third ventricle, VPS was performed in this patient, but not etV. All the patients were doing well during follow-up (mean 68, range 6-149 months).

Conclusion: The treatment outcomes of $\mathrm{OH}$ associated with UBO in NF1 were good using either VPS or etV. However, the treatment procedures, VPS or etV, should be selected based on the obstructive patterns.

\section{2}

\section{Safety and efficiency of endoscopic neurosurgery in children -} experience with 164 procedures

Meike K. Petri ${ }^{1}$, Christoph A. Tschan ${ }^{1}$, Henry W. S. Schroeder ${ }^{2}$, Sonja Vulcu ${ }^{1}$, Michael R. Gaab ${ }^{3}$, Joachim Oertel ${ }^{1}$

${ }^{1}$ Department of Neurosurgery, Universitätsklinik des Saarlandes; ${ }^{2}$ Department of Neurosurgery, Ernst Moritz Arndt University, Greifswald; ${ }^{3}$ Department of Neurosurgery, Nordstadtkrankenhaus Hannover

Object: Endoscopic surgery in children has particular characteristics and is associated with different rates of success. In this study we would like to figure out the ideal indications and safety of neuroendoscopic procedures in children.

Methods: Success rate of 164 procedures was evaluated according to pathology, type of procedure and influence of patients' age. Endoscopy was used in 54 brain tumours, 33 aqueductal stenosis, 29 cystic lesions, 21 various malformations, 7 haemorrhagic and 7 isolated ventricles. Data were analysed focused to clinical and radiological success as well as shunt dependence.

Results: The follow-up period ranged from 6 months to 6 years. The success rate of CSF circulation restoration was $72 \%$. An intraoperative switch to microsurgery was necessary in 3 of 164 operations. Patients younger than 6 months at the time of surgery became more frequently shunt dependent than older children. The best outcome and the lowest complication rate were obtained in endoscopy cyst fenestration procedures. Conclusion: Based on this data analyses acute occlusive hydrocephalus is best indication for neuroendoscopy in children. Summarized, endoscopy can be considered efficient and save in children.

\section{3}

Endoscopic third ventriculostomy for post-inflammatory hydrocephalus: Is it worth to try?

Alaa A. Raouf

Department of Neurosurgery, Alexandria University, Egypt

Introduction: Hydrocephalus is a very common disease in developing countries. Till the recent introduction of cranial endoscopy and In spite of their frequent complications, shunts were considered as the commonest treatment option for this disease condition. Endoscopic third ventriculostomy - especially for obstructive hydrocephalus - opened a new era of treatment that is free of the lifelong shunt dependency. Better results were obtained with obstructive rather than with post inflammatory hydrocephalus or in cases with Chiari malformation. Recent reports of trying this procedure in post inflammatory hydrocephalus are increasing. Methods: We tried endoscopic third ventriculostomy as a first line therapy in 35 children with hydrocephalus proved to be secondary to intracranial infection. Patients with non settled inflammatory etiology and those with complex septated hydrocephalus were excluded from the study. The rigid Gaab system was used in all cases.

Results: The aqueduct was obstructed in 11 patients. The overall improvement was obtained in $55.9 \%$. Cases with aqueductal obstruction showed procedure success in $81.9 \%$. Procedure related complications were not encountered.

Conclusion: ETV is a simple safe and effective method in treating not only obstructive but also post inflammatory hydrocephalus especially when the aqueduct is obstructed. An overall fifty percent improvement in our study and even more in other series encourage even the trial of getting rid of the lifelong shunt complications and suffering.

\section{4}

Endoscopy-, ultrasound- and laser-assistance in pediatric MIN: Application in preterm twins

Klaus D. M. Resch ${ }^{1}$, Peter T. Ulrich ${ }^{1}$, Georg Frey ${ }^{2}$

${ }^{1}$ Neurosurgery, ${ }^{2}$ Neonatology, Klinikum Darmstadt, Germany

Introduction: Consequently realization of the "key-hole concept" is still a challenge due to many technical problems. We investigated the combined use of ultrasound, endoscopy and laser in a first series. Application in preterm twins, a regular and a complex case, shall illustrate the benefit of this concept.

Material and Methods: The Almikro-endoscopy system with HD video tower, the ALOKA Alfa 7 high-end ultrasound equipment and the LISA Revolix Th:YAG-Laser was used in different combination in a series of 30 complex cases, among which in preterm twins with connatal hydrocephalus it was applied.

Results: In the preterm twins this concept was successfully realized: one case had regular clinical course the other showed a very complex course. In the latter the difficult multicystic problems were solved. Obviously without this concept these danger reaction with rising ICP could not be controlled. Conclusion: Realization of the "key-hole concept" needs combination of techniques that mutual compensate the weaknesses. Endoscopy, ultrasound and laser complete one another. Precise planning and training are mandatory for clinical use and good results. This concept seams promising for preterm complex hydrocephalus cases.

\section{5}

The ShuntScope - new technique for catheter placement in complex cases of hydrocephalus in pediatric neurosurgery

Mohamed Salah, Joachim Oertel, Christoph A. Tschan

Department of Neurosurgery, Saarland University Hospital and Saarland University Faculty of Medicine, Homburg/ Saar, Germany

Objective: The long-term preserving of ventriculoperitoneal shunt function depends on the correct placement of the ventricle catheter. Nearly $4 \%$ of ventricular catheters are misplaced. There are already many tools to optimize the insertion, such as neuronavigation or stereotactic techniques. Methods: We used the new semirigid ShuntScope (Karl Storz, Germany) for the catheter placement in cases of slit ventricles, pseudotumor cerebri, cystic lesions and in cases of aqueductoplasty. The study was carried on 33 patients (19 males, 14 females), mean age 40 (5 to 78 years, 7 children). 24 patients presented with slit venticles or difficult anatomic ventricular configurations. The ShuntScope was used diagnostic and therapeutic. The small outer diameter of $1 \mathrm{~mm}$ allows to pass through 
the catheter under directly endoscopic view with a very good image resolution of 10000 Pixels.

Results: The main value of this new technique was the ability to place the catheter tip accurately within distorted or small ventricles. Even controlled and guided placements in the fourth ventricle were possible in cases of aqueductoplasty. The semirigid endoscope allowed a safe intraoperative correction of the catheter placement due to a cover sheet function of the ventricular catheter. The postoperative neuroradiological imaging revealed the catheter tip placement analogous to the intraoperative findings and video recording analysis. No bleeding complications or infections occurred.

Conclusion: The ShuntScope is very useful for safe catheter placement in complex cases of hydrocephalus. With this new technique misplacements of shunt catheters are completely avoidable. The ShuntScope technique should be implemented in Pediatric Neurosurgery.

\section{6}

Neuroendoscopy in the management of paediatric hydrocephalus outcome analysis in 94 procedures

Sérgio Salvador ${ }^{1,2,3}$, Joana Oliveira ${ }^{1,2}$, Josué Pereira ${ }^{1,2}$, Rui Vaz ${ }^{1,2}$

${ }^{1}$ Serviço de Neurocirurgia do Hospital de São João, Porto, Portugal;

${ }^{2}$ Faculdade de Medicina da Universidade do Porto, Porto, Portugal;

${ }^{3}$ Faculdade de Ciências de Saúde da Universidade Lúrio, Nampula, Moçambique

Introduction: Nowadays, the endoscopic third ventriculostomy (ETV) is the treatment of choice for obstructive hydrocephalus, but the outcome stills controversial with regard to age and cause.

Methods: Between 1998 and 2011, 94 procedures (in 91 patients) were performed in pediatric patients $(56.4 \%<2$ years of age), with a malefemale rate of $2: 1$. There were no patients lost to follow-up, in period since march 19th 1998, until December 31st of 2012 (13 to 165 months). Where include all consecutive paediatric cases of obstructive hydrocephalus, submited to etV. Successful etV was defined as resolution of symptoms with shunt independence.

Results: ETV was successful in $67.0 \%$ of all the patients (tumors $95.8 \%$, Chiari malformation $40.0 \%$, aqueductal stenosis $66.7 \%$, post-infectious $50 \%$, post-hemorragic $42.9 \%$ and VP shunt malfunction $56.0 \%$ ), and $57.7 \%$ in younger than 2 years. $26.6 \%$ needed VP shunt before 1 month after etV and $6.4 \%$ needed VP shunt after 1 month after etV. Three patients were subjected to a new etV for closure of stoma, on average 3.3 years ( 2 months to 9 years) after the first procedure with $100 \%$ effectiveness. There were no post-operative infections.

Conclusions: ETV is a safe and effective treatment for obstructive hydrocephalus, even in case of disfunction of VP shunt. Although the poor etV outcome in the very young group, it represent more than $50 \%$ of shunt disease free children. We recommend etV as the first option in obstructive hydrocephalus, in all the etiological groups no matter the age.

\section{7}

\section{Evaluation of endoscopic intraventricular fenestrations} in combination with other endoscopic procedures

Sebastian Senger, Sonja Vulcu, Joachim Oertel

Department of Neurosurgery, Saarland University Medical Center and Saarland University Faculty of Medicine, Homburg Saar, Germany

Background: Tumors and cysts of the inner liquor system or those in direct adjacency to it are a surgical challenge due to their localization. These formations often go in hand with hydrocephalic symptoms caused by occlusion or compression of the liquor circulation. The endoscopic transventricular surgery is supposed to handle both, hydrocephalic treatment via third ventriculostomy or cyst fenestration and adequate minimal invasive tumor surgery in one procedure.

Methods: 75 Patients (34 male, 31 female) from the age of 1 to 71 underwent endoscopic ventriculcisternostomy or ventriculoseptostomy in combination with the resection or biopsy of an intraventricular cyst or tumor. All patients suffered from hydrocephalic symptoms or neurological deficits caused by tumors/cyst of the inner liquor system or by tumors in direct adjacency.

Results: The operation was performed via a single burr hole almost in every case. Only in one cases a second burr hole was necessary to reach the tumor. In no cases a conversion to open microsurgery was required. Bleedings occurred during biopsy in some cases, always under intraoperative haemostasis.

Conclusion: As described above the endoscopic treatment of these tumors is a safe and reliable technique. Due to its small access point and enhanced intraoperative view endoscopy fulfills all requirements for a minimal invasive and safe operation technique. It will be even improved in the next years with new developments in endoscopic tools and intraoperative navigation.

\section{8}

Telemetric long-term ICP monitoring in children

Christoph A. Tschan ${ }^{1}$, Sebastian Antes ${ }^{1}$, Wolfgang Wagner ${ }^{2}$, Joachim Oertel $^{1}$

${ }^{1}$ Department of Neurosurgery, Saarland University Hospital and Saarland University Faculty of Medicine, Homburg/Saar, Germany; ${ }^{2}$ Department of Neurosurgery, Section of Pediatric Neurosurgery, University Hospital of Mainz, Mainz, Germany

Introduction: Monitoring of intracranial pressure (ICP) is usually performed during hospital stay by using percutaneous intraparenchymal catheters or ventricular drains. First prototypes of wireless measurement devices have been developed in the late Sixties. However, almost all devices in the past could not assert themselves due to technical restrictions and measuring inaccuracies. In January 2010, a new promising telemetric ICP monitoring system was commercially introduced to the market. Here, the first long-term experiences in children are presented.

Methods: The telemetric intraparenchymal pressure probe (RAUMEDIC Neurovent P-tel) was applied in 26 children (mean age: 10.5 years, range: 1.5 to 18.0 years). In 24 children long-term ICP home-monitoring was performed. Main indications for ICP measurements were suspected hydrocephalus, exclusion of shunt dysfunction, monitoring after shunt ligature and patency control after neuroendoscopy. Additionally, telemetric technique supported individual postoperative valve adjustments in shunt-treated children.

Results: Mean duration of surgical insertion was 18 mins. ICP monitoring was averagely performed for 61 days (range: 8 to 209 days). In all 26 children, there have been no periprocedural complications. Pathological ICP values could be detected in 17 patients entailing further neurosurgical therapy. Overall, the telemetric system was well tolerated and easy to handle by parents and children. The zero drift of the telemetric pressure probe was between -2 and $+2 \mathrm{mmHg}$.

Conclusions: The new telemetric system allows safe and reliable longterm ICP measurements in pediatric neurosurgery. The possibility of performing uncomplicated home-monitoring increases decisively the tolerance of patients and parents.

\section{9}

Recurrent signs of elevated intracranial pressure in a 14-year-old girl with a pineal cyst without hydrocephalus: Correlation of clinical symptoms with increases of intracranial pressure measured by a telemetric ICP probe during non-invasive home monitoring Wolfgang Wagner ${ }^{1}$, Alexandra Huthmann ${ }^{1}$, Caroline Hofmann ${ }^{2}$ ${ }^{1}$ Section of Pediatric Neurosurgery, Department of Neurosurgery, ${ }^{2}$ Section of Neuropediatrics, Childrens' Hospital, University Medical Center Mainz, Germany 
A 14-year-old healthy girl presented with recurrent attacks of severe headaches, dizziness and collapse. These symptoms occurred without warning signs, lasted a few seconds or minutes and resolved without special treatment. A cranial MR showed a pineal cyst with slight compression of the (still patent) aqueduct and no hydrocephalus. Neurological and fundoscopic examination were perfectly normal. Control MR three months later and repeated fundoscopy were identical. Extended neurologic examination during a three weeks hospital stay excluded other causes of the attacks. A telemetric ICP probe (Raumedic AG, Helmbrechts, Germany) was implanted. Long-term ICP monitoring at home revealed that the described attacks were regularly accompanied by elevated ICP values $(>30 \mathrm{~mm} \mathrm{Hg})$; as for the rest, ICP was normal $(<10 \mathrm{~mm}$ $\mathrm{Hg}$ ). Assuming that an intermittent occlusion of the aqueduct lead to ICP increases without ventricular enlargement, we performed a microsurgical fenestration of the pineal cyst via a supracerebellar infratentorial approach. Postoperative MR showed a collapse of the cyst and an absolutely free aqueduct. The described attacks completely disappeared (follow-up 3 months). ICP monitoring at home no longer showed ICP increases. Surgery of small pineal cysts with a patent aqueduct is not generally recommended. However, it is known that fenestration of such cysts may relieve symptoms of increased ICP even in patients without hydrocephalus. This case study demonstrates for the first time by long term ICP home monitoring pressure increases without ventricular enlargement leading to clinical attacks that completely disappeared after microsurgical fenestration of the cyst.

\section{0}

Could hydrocephalic infants benefit from ventriculo-peritoneal shunt with an "ultra-high" pressure valve with respect to prevention of both future slit ventricle syndrome and proximal obstruction? 12 years experience in the pediatric neurosurgery center in Shanghai, China

Bo Xiao, Jiang-Gang Liu, Yu Liu, Li-Hao Fang, Ruo-Ping Chen

Neurosurgery Department, Shanghai Children's Hospital, China

Object: The aim of the present study was to determine the association between the pressure of valve implanted in pediatric hydrocephalic patients $\leq 1 \mathrm{yr}$ and future slit ventricle syndrome (SVS)/shunt proximal obstruction (SPO)

Methods: We conducted a retrospective cohort review of hydrocephalic cases with their first VP shunt inserted $\leq 1 \mathrm{yr}$ (since 2001 to 2007) and follow-up for $\geq 6$ years. Additional exclusive criteria for patient selection were set. Patients included were categorized into 3 groups based on their initial valve pressure: Group A $\left(50 \sim 90 \mathrm{~mm} \mathrm{H}_{2} \mathrm{O}\right)$, Group B $(100 \sim$ $150 \mathrm{~mm} \mathrm{H}_{2} \mathrm{O}$ ) and Group $\mathrm{C}\left(\geq 150 \mathrm{~mm} \mathrm{H}_{2} \mathrm{O}\right.$ achieved using HAKIM ${ }^{\circledR}$ Programmable Valve). Criteria of SVS/SPO were given. Cortical mantle thickness on follow-up radiological imagings were measured.

Results: A total of 103 cases were included (47, 32 and 24 cases in Group A through $\mathrm{C}$, respectively). Complication rate of SVS/SPO in Group C was significantly lower than that in other 2 groups with both $p<0.05$. When valve pressure was $\geq 150 \mathrm{~mm} \mathrm{H}_{2} \mathrm{O}$, positive likelihood ratio for future SVS and SPO was $0.32(\mathrm{p}<0.05)$, while it was $0.66(\mathrm{p}>0.05)$ when valve pressure was $\geq 100 \mathrm{~mm} \mathrm{H}_{2} \mathrm{O}$. There was no significant difference among patients in Group A, B, and C with respect to their cortical mantle thickness. Conclusion: Pressure of valves implanted in those patients was highly associated with incidence of SVS/SPO in an inverse way. To avoid complications of SVS/SPO, initial pressure set $\geq 150 \mathrm{~mm} \mathrm{H}_{2} \mathrm{O}$ was recommended.

\section{Infection}

\section{1}

Intracranial echinococcosis in pediatric patient - Case report and illustrative video

Cristina B. Bleil ${ }^{3}$, Jorge W. J. Bizzi ${ }^{1}$, André Bedin ${ }^{2}$, Fabiano P. Soares ${ }^{3}$, Maira C. Velho ${ }^{3}$, Rodrigo Roncaglio ${ }^{3}$, Marcelo C. Vione ${ }^{3}$
${ }^{1}$ Chief of Pediatric Neurosurgery Department, Hospital da Crianca Santo Antonio, Porto Alegre, Brazil; ${ }^{2}$ Neurosurgeon at Pediatric Neurosurgery Department, Hospital da Crianca Santo Antonio, Porto Alegre, Brazil; ${ }^{3}$ Resident at Pediatric Neurosurgery Department, Hospital da Crianca Santo Antonio, Porto Alegre, Brazil

Introduction: Cystic equinococcosis is a parasitic disease, typically seen in rural areas. It is an endemic zoonotic infection in Southern Brazil and must be considered in differential diagnosis of intracranial cystic lesions. We present a case of a 9 yo male with a large left front-parietal hydatid cyst and its treatment

Case Report: Male, 9 yo, from rural area, was referred to neurosurgical evaluation at Regional Pediatric Hospital due to intracranial mass lesion on a CT scan. The child suffered initially from impaired learning and later with gait disturbance and mild right arm paresis. Initial cranial CT scan evaluation showed a left front-parietal cystic lesion with midline shift. Abdominal CT scan showed a right lobe cystic hepatic lesion. Cranial MRI demonstrated a hypointense T1/hyperintense T2 lesion, with mild gadolinium peripheral enhancement, measuring $7,9 \times 7,8 \mathrm{~cm}$. The patient underwent surgical removal according to Dowling technique without any complication. Postoperative recovery was uneventful. Nine days after, the child was submitted to hepatic cyst resection and was released from hospital in 18 days. Anatomopathological analysis confirmed initial hypothesis of Hydatid Cyst. Patient fully recovered from neurological symptoms.

Conclusion: Echinococcosis is still common in countries underdeveloped. Clinical treatment with antihelmintic agents is less effective, reserved for recurrent or inoperable cysts. Transoperative cyst rupture has high risk of anaphylaxis. Cyst resection is the treatment of choice. Dowling-Orlando technique and its variations, such as PAIR (percutaneous aspiration, injection and reaspiration) are useful, low cost and effective surgical strategies.

\section{2}

The use of neuroendoscopy in the treatment of repeated ventriculitis Daniel D. Cardeal, Leonardo M. Sousa, Jr., Fernanda G. Andrade, Hamilton Matushita

Hospital das Clinicas, University of Sao Paulo

Introduction: Ventriculoperitoneal shunt (VPS) is the main form of treatment of hydrocephalus in children. The VPS infections may be difficult to treat. Most neurosurgeons choose to remove the shunt and use systemic or intraventicular antibiotics and external ventricular shunt (EVS) before implanting a new VPS. In some cases, ventriculites become refractory requiring long period of antibiotic treatment. We evaluated the use of neuroendoscopy in the treatment of refractory ventriculites.

Methods: Seven children with repeated ventriculitis were submitted to neuroendoscopy with exhaustive "washing" of ventricular cavity. We performed endoscopic fenestration in cases of multiple septations and, if possible, ventriculostomy with choroid plexus coagulation. We analyzed the cerebrospinal fluid and evolution of the infection after the procedure.

Results: Six patients were under 15 months of age and one was 12 years old. Six patients had ventriculitis related to VPS infection and one as a complication of meningitis. The etiologic agent was identified in 4 cases: 2 cases staphylococcus aureus, 1 case staphylococcus epidermidis and 1 case pneumococcus. Empirical antibiotics were the choice in cases of negative cultures. The cases were treated for about 3 weeks with EVS and systemic antibiotics without satisfactory improvement of the CSF. There were no procedure-related complications. The follow-up ranged from 4 months to 1 year. In all cases there was an improvement of the CSF after the procedure. Six patients were discharged; one patient died due to clinical complications.

Conclusion: Neuroendoscopy is a safe procedure and helps the treatment of refractory ventriculitis decreasing hospitalization and antibiotic treatment. 
83

Long, subcutaneous tunnelled external ventricular drains, a novel approach to reduce infection rates and inadvertent dislodgement. A retrospective study

Christian D. E. Collins ${ }^{1}$, John C. Hartley ${ }^{2}$, Dominic N. P. Thompson ${ }^{3}$, Aabir Chakraborty ${ }^{3}$

${ }^{1}$ Medical Student, University of Southampton; ${ }^{2}$ Department of Microbiology, Great Ormond Street Hospital, London, UK; ${ }^{3}$ Department of Neurosurgery, Great Ormond Street Hospital, London, UK

Introduction: Short-tunnelled EVD insertions are associated with complication rates of up to $30 \%$. We hypothesise that this rate can be reduced by the use of long-tunnelled EVDs (LTEVD). This study presents our experience with LTEVDs.

Methods: A retrospective case notes audit of patients who had insertion of LTEVDs from 2008 to 2012 was performed. Reason for insertion, infection rate following LTEVD insertion, duration of insertion and inadvertent dislodgement rates requiring reinsertion, were calculated. The number of patients who required an additional procedure to remove the LTEVD not associated with any other procedure such as internalisation of VP shunt was calculated. Data were compared to other published series.

Results: 181 LTEVDs were inserted. The mean age was 6.6 years (range $0-15.5$ years). Reasons for insertion included intra-ventricular haemorrhage $(47 \%)$, infection $(27 \%)$, tumour related hydrocephalus $(7.2 \%)$, as a temporising measure $(17 \%)$ and trauma $(2.2 \%)$. The infection rate was $2.76 \%$. Mean duration of insertion was 10 days (range $0-42$ days). 4 LTEVDs $(2.2 \%)$ were inadvertently dislodged requiring reinsertion. 13 patients $(7 \%)$ required removal of EVD alone. There was a significant difference $(\mathrm{P}<0.05)$ when comparing our infection rate to 15 publications of infection rates in short-tunnelled EVDs, however there was no difference when comparing our data to 3 publications using LTEVDs. The literature suggests an EVD dislodgement rate of $8.1 \%$. Conclusions: LTEVDs reduce infection rates, have low dislodgement rates requiring an extra procedure, but require $7 \%$ more procedures for removal when compared to short-tunnelled EVDs. LTEVDs should be considered in all patients where temporary CSF diversion is required.

\section{4}

Pediatric parafalcine empyemas: a rare entity, case description and review of the literature

Franziska Niklewski ${ }^{1}$, Athanasios K. Petridis ${ }^{1}$, Jasmin Al Hourani ${ }^{1}$, Klaus Blaeser ${ }^{1}$, Georgios Ntoulias ${ }^{1}$, Andrej Bitter ${ }^{1}$, Thorsten Rosenbaum $^{2}$, Martin Scholz ${ }^{1}$

${ }^{1}$ Department of Neurosurgery, Academic Teaching Hospital of University Duisburg-Essen, Wedau-Kliniken, Duisburg, Germany; ${ }^{2}$ Departement of Pediatrics, Academic Teaching Hospital of University Duisburg-Essen, Wedau-Kliniken, Duisburg, Germany

Introduction: Subdural intracranial empyemas and brain abscesses are a rare complication of bacterial sinusitis and must be treated immediately. Parafalcine abscesses in children are rarely described and seem to show differences in their treatment compared to other brain abscesses. We present two pediatric cases, in which children developed a falcine abscess as a complication of sinusitis and introduce our department's treatment management. In addition we performed a review of literature concerning the management of pediatric falcine empyemas.

Methods: We searched pubmed for articles referring to brain abscess (9694) and continued our research looking for „,brain abscesses in children“(2056) and ,interhemispheric abscesses in children“(13). Another path was looking for subdural empyemas (921), subdural empyemas in children (331). Four articles concerning to parafalcine subdural empyemas in children were found. We describe two cases of parafalcine abscesses in children as a complication of sinusitis frontalis.
Results: Intracranial evacuation of pus seems to be the surgical treatment of choice in parafalcine subdural empyemas and abscesses in children. An additional therapy with antibiotics is needed. In our cases both of the children showed a recurrent empyema after the first surgical treatment and antibiotic therapy. A second surgical evacuation was necessary. The antibiotic therapy was given for three months.

Conclusion: Short time follow-up imaging is necessary irrespective of infection parameters in blood and patient's clinical condition. Especially in parafalcine abscesses a second look may be an option and surgical treatment with evacuation of pus is the treatment of choice if abscess remnants are visualized.

\section{Oncology}

\section{5}

IDH1 mutations with relevance to $1 \mathrm{p} / 19 \mathrm{q}$ loss and MGMT promoter methylation in pediatric glioma patients

Jun-ichi Adachi, Takaaki Yanagisawa, Tomonari Suzuki, Kohei Fukuoka, Mitsuaki Shirahata, Kazuhiko Mishima, Ryo Nishikawa

Department of Neuro-Oncology/Neurosurgery, Saitama Medical University International Medical Center, Saitama, Japan

Purpose: Isocitrate dehydrogenase 1 (IDH1) mutations are genetic alteration with a high prevalence $(50-70 \%$ of cases) in WHO grade II or III gliomas and secondary glioblastoma. The acquisition of IDH1 mutations occurs early in the development of a glioma from a glial progenitor cell that can give rise to both astrocytes and oligodendrocytes. These results, however, are based on studies of adult gliomas, but not pediatric gliomas. In this study, we examined IDH1 mutations in pediatric glioma patients with the following goals: 1) to compare the prevalence of IDH1 mutations in pediatric gliomas with those of adult ones; 2 ) to evaluate the relationship between IDH1 mutations and loss of $1 \mathrm{p} / 19 \mathrm{q}$ or $\mathrm{O}_{6}$-methylguanineDNA methyltransferase (MGMT) promoter methylation.

Material and methods: We analyzed the mutational hot spot, codon 132 of IDH1 in 26 newly diagnosed pediatric gliomas. Real-time PCR system followed by high-resolution melting (HRM) analysis were used to detect IDH1 mutaions. We estimated loss of $1 p / 19 q$ and MGMT methylation status by FISH and methylation specific HRM, respectively.

Results: Of the 26 cases, IDH1 was mutated in only one glioma (anaplastic astrocytoma). This case was accompanied with methylated MGMT. Four pediatric oligodendrogliomas with co-deletion of $1 p / 19 q$ had no IDH1 mutations and no methylated MGMT.

Conclusions: The frequency of IDH1 mutations in pediatric gliomas was significantly low irrespective of tumor grades or histology. All 1p/19q codeleted adult oligodendrogliomas showed IDH1 mutations, whereas pediatric oligodendrogliomas did not. These results suggest that there is a difference between pediatric gliomas and adult ones in genetic significance of IDH1 mutations in gliomagenesis.

\section{6}

Neurosurgical management of neurenteric cysts in children Ibrahim H. Al-Ahmed, Mohamed Boughamoura, Peter Dirks, Abhaya V. Kulkarni, James T. Rutka, James M. Drake

Division of Neurosurgery, The Hospital for Sick Children, The University of Toronto

Background: Neurenteric Cysts are endothelium-lined structures of presumed endodermal origin. There have been few pediatric series of intracranial neurenteric cysts reported previously. We present our experience in the management of these lesions.

Methods: A retrospective chart review of all patients identified with neurentric cysts between 1977-2007 was carried out. Demographics, details of clinical presentation, surgical therapy, and outcome data was extracted. 
Results: Six girls and 5 boys with average age of 4.6 yrs (range $0-14$ yrs) were identified. Limb weakness was the most common presenting symptom. Location of the cysts was cervical in 3 cases, cervicothoracic in 3 cases and thoracic in 3 cases. One cyst was anterior to the pons with extension to the left cerebellopontine angle (CPA) and one case was at the craniocervical junction. Five patients had an apparently initial complete excision with one recurrence. Four patients were initially treated with incomplete excision and/or cyst drainage usually into an Ommaya reservoir. A newborn with a large cervico-thoracic cyst died of multiple congenital anomalies without any intervention. All other patients are alive at last follow-up. One patient with a presumed radiologically identified cyst which remained stable was simply followed. Several patients required multiple procedures and one patient developed hydrocephalus after aseptic meningitis and was shunted.

Conclusion: Neurenteric cysts are rare in the pediatric population. Total resection, if possible provides the best long term outcome. Incomplete excision may lead to multiple procedures, including cyst drainage, but can lead to long-term stability.

\section{7}

\section{Papillary tumor of the pineal region}

Romina A. Argañaraz, Graciela N. Zúccaro

Department of Neurosurgery, Hospital Nacional de Pediatría "Juan P. Garrahan", Buenos Aires, Argentina

Papillary tumors of the pineal region (PTPR) represent a rare and histologically distinct subgroup of tumors originating in this area included in the 2007 WHO classification as grade II/III due to local recurrence. Between 1988 and 2012, we operated on 98 pineal tumors, three of which, initially misdiagnosed, turned out to be PTPR.

Case1: A 8-year-old girl consulted for tremor. On MRI a heterogeneous contrast-enhancing mass was found in the pineal region. The tumor, diagnosed as papillary pineocytoma, was totally removed in 2002. In 2004 , the tumor recurred and was subtotally removed. Radiotherapy was indicated. The girl died 11 years after diagnosis due to tumor recurrence. Case 2: A 4-year-old girl with ICP. The MRI showed heterogeneous tumor in the pineal region and hydrocephalus. ETV and biopsy were performed. Diagnosed as a papillary ependymoma, the tumor was subtotally resected. Radiotherapy was indicated. She had been tumor free for 5 years when tumor recurred with metastasis. she underwent chemotherapy, but died 4 years later.

Case 3: A 12-year-old boy with ICP and visual disturbances. On MRI, hydrocephalus and a heterogeneous mass in the pineal region were seen. After etV and biopsy, undifferentiated tumor was diagnosed and radiotherapy was indicated. After 3 years the tumor recurred and was subtotally removed. Diagnosis was PTPR and radiosurgery was indicated. Due to tumor regrowth, he received chemotherapy over 3 years. Ten years after initial diagnosis, the boy is alive without sequelea but with stable tumor. Conclusion: PTPR is extremely rare, but should be considered in the differential diagnosis of pineal tumors. Patients should be carefully followed-up, because prognosis is still unknown and local recurrence is frequent.

\section{8}

Ependymomas in pediatric population in Southern Brazil - Eighteen years experience

Cristina B. Bleil ${ }^{3}$, Jorge W. J. Bizzi ${ }^{1}$, André Bedin ${ }^{2}$, Fabiano P. Soares ${ }^{3}$, Maira C. Velho ${ }^{3}$, Rodrigo Roncaglio ${ }^{3}$, Marcelo C. Vione ${ }^{3}$

${ }^{1}$ Chief of Pediatric Neurosurgery Department, Hospital da Crianca Santo Antonio, Porto Alegre, Brazil; ${ }^{2}$ Neurosurgeon at Pediatric Neurosurgery Department, Hospital da Crianca Santo Antonio, Porto Alegre, Brazil; ${ }^{3}$ Resident at Pediatric Neurosurgery Department, Hospital da Crianca Santo Antonio, Porto Alegre, Brazil

Introduction: Ependymomas represents $7 \%$ of all central nervous system neoplasms in pediatric population. Despite the advances in technology, neuroimaging and neuroncology the treatment of ependymomas remains a great challenge. Many controversial issues are still debated, such as the influence of histological grade, biological markers, adjuvant therapy, proliferation index, location of the tumor. The aim of this study is to analyzethe epidemiologic features and outcomes in children treated in our institution. Methods: We reviewed medical records of 40 children diagnosed with ependymomas at the first surgical resection treated at Hospital da Crianca Santo Antonio between august 1995 to January 2013. Statistical analysis was performed with SPSS ${ }^{\circledR} 20$, using Fischer and chi-square tests. Results: Patients were predominantly male $(60 \%)$, average age at diagnosis was 4 yo. All of them underwent surgery, and gross total resection was achieved in $57 \%$ patients; there were $50 \% \mathrm{WHO}$ grade II and $50 \% \mathrm{WHO}$ grade III at the time of the first resection. Major presentation was intracranial hypertension due to obstructive hydrocephalus in $45 \%$ of patients. During follow-up, 50\% needed hydrocephalus treatment. Four patients with WHO grade II had progression and 1 had spinal metastasis. Followup was from 1 month old to 17 yo (41,4 months). There was no difference between survival analysis and WHO grade classification. Medium survival time in patients WHO grade II who died during analysis was 19 month versus 39,5 month in patients WHO grade III.

Conclusion: Despite efforts in improving neurosurgical and oncology treatments, ependymomas has limited prognosis in pediatric population.

\section{9}

Surgical treatment of medulloblastoma in children

Cristina B. Bleil ${ }^{3}$, Jorge W. J. Bizzi ${ }^{1}$, André Bedin ${ }^{2}$, Fabiano P. Soares ${ }^{3}$, Maira C. Velho ${ }^{3}$, Rodrigo Roncaglio ${ }^{3}$, Marcelo C. Vione ${ }^{3}$, Carolina Klovan ${ }^{4}$ ${ }^{1}$ Chief of Pediatric Neurosurgery Department, Hospital da Criança Santo Antonio, Porto Alegre, Brazil; ${ }^{2}$ Neurosurgeon at Pediatric Neurosurgery Department, Hospital da Criança Santo Antonio, Porto Alegre, Brazil; ${ }^{3}$ Resident at Pediatric Neurosurgery Department, Hospital da Criança Santo Antonio, Porto Alegre, Brazil; ${ }^{4}$ Physician

Introduction: Medulloblastomas are known to be the one of the most frequent intracranial neoplasm in children. Prognostic factors are related to surgical resection and adjuvant treatment. The aim of this study is to review a single surgeon experience over fifteen years.

Methods: We reviewed the medical records of 50 patients with medulloblastoma operated during the period 1995 to 2009 in Santo Antônio Children's Hospital. Using a protocol, we observed the clinical and demographic factors of the disease, with its corresponding influence on patient survival. Overall survival was measured by the Kaplan-Meier curve and statistical significance of the variables was observed by Fisher and Qui-square tests.

Results: The mean age of diagnosis was 80.6 months $( \pm 42.9)$, occurring predominantly in males $(57.4 \%)$. The presentation of the disease proved by increased intracranial pressure $(97.9 \%)$ and cerebellar syndrome $(57.4 \%)$. Complete surgical resection of the tumor was possible in $80.4 \%$ of cases. The overall survival at three years was $49.5 \%$. In patients whose tumor resection was complete the overall survival rate was higher than in patients with parcial resection $p=0,002$ ) Most common surgical complications were cranial nerve palsy and cerebrospinal fluid leak.

Conclusion: The aggressive surgical treatment, aiming total resection of the tumor proved to be the main factor for favorable prognosis.

90

Leptomeningeal enhancement as a diagnostic and prognostic indicator in atypical Teratoid/Rhabdoid tumor

Ira Bowen ${ }^{1}$, Yasser Jeelani ${ }^{2}$, Stephanie Da Silva ${ }^{2}$, J. Gordon McComb ${ }^{2}$, Mark D. Krieger ${ }^{2}$

${ }^{1}$ West Virginia School of Osteopathic Medicine, Lewisburg, WV; ${ }^{2}$ Division of Neurosurgery, Children's Hospital of Los Angeles, Los Angeles, CA Department of Neurological Surgery, Keck School of Medicine, University of Southern California, Los Angeles, CA 
Introduction: Atypical Teratoid/Rhabdoid Tumors (AT/RT) are a rare primary CNS tumor of childhood. Although they can be indistinguishable from medulloblastomas on imaging, AT/RTs tend to have a poorer prognosis.

Methods: This IRB-approved study retrospectively reviewed children with AT/RT between 2000 and 2012 at our institution. Pre and postoperative MRIs were evaluated for tumor size and location, leptomeningeal enhancement, extent of resection, and presence of calcification, cysts, hemorrhage, and necrosis. Results were compared with 83 age matched patients with medulloblastoma. Magnetic Resonance Spectroscopy was available for 9 of the AT/RT patients.

Results: 24 children had an average age at diagnosis of 4.8 years (range 3 $211 \mathrm{mo}) ; 11 / 24$ (46\%) were female. Mean overall follow-up for the 2 groups was 23 months for AT/RT and 42 months for medulloblastoma. The average overall survival (OS) for AT/RT patients was 25 months vs. 41 months for medulloblastoma patients $(\mathrm{p}=0.03)$. The OS for males with AT/RT was 17 months vs 39 months for females $(\mathrm{p}=.05) .8 / 24(33 \%)$ AT/ RTs presented with leptomeningeal enhancement vs $8 / 83(9 \%)$ in medulloblastomas. No significant difference was seen in the OS in AT/RT patients presenting with leptomeningeal enhancement vs those without. Conclusion: AT/RTs are more likely to have leptomeningeal enhancement at presentation compared with medulloblastoma. As expected, prognosis for AT/RT was statistically worse than for medulloblastoma. While leptomeningeal enhancement was more likely to occur in AT/RT it did not prove indicative of a poorer prognosis.

\section{1}

Chorionic gonadotrophin producing brain germinoma as a cause of precocious pseudopuberty

Eva Brichtova ${ }^{1}$, Zdenek Pavelka ${ }^{2}$, Olga Magnova ${ }^{3}$, Leos Kren ${ }^{4}$, Tomas Svoboda $^{5}$, Karel Zitterbart ${ }^{2}$, Jaroslav Sterba ${ }^{2}$

${ }^{1}$ Department of Pediatric Neurosurgery, ${ }^{2}$ Department of Pediatric Oncology, ${ }^{3}$ Pediatric Department, ${ }^{4}$ Institute of Pathology, ${ }^{5}$ Department of Neurosurgery, University Hospital Brno and Faculty of Medicine, Masaryk University, Brno, Czech Republic

Intracranial germ cell tumors represent $3-5 \%$ of primary central nervous system tumors in children, in 50-60\% consist of germinomas. The authors present a case report of 6-year-old boy with a history of Langerhans cell histiocytosis with subsequent brain germinoma, clinically manifested by precocious pseudopuberty on the basis of chorionic gonadotrophin ectopic secretion. The germinoma was verified histologically after stereotactic biopsy and classified as "pure germinoma". The biopsy was performed due to atypical and rare, but possible tumor localization in basal ganglia on the right and serum chorionic gonadotrophin values below the diagnostic level for secretoric non-germinomas. Due to the presence of two different malignancies, the patient underwent genetic examination including suppressor and DNA repair genes mutations screening. The child started treatment according to ACNS 0232 protocol. The chemotherapy by BDCA/VP16 and CDDP/cyclophosphamide cycles were followed by response differentiated radiotherapy. To blockade testosterone effect on target receptors, anti-androgen cyproterone acetate was deployed. Due to the association with second malignancy the genetic background is possible and the prognosis remains uncertain. The case demonstrates the need for an interdisciplinary approach to diagnosis and treatment of such pediatric pateints. Differential diagnosis of chorionic gonadotrophin producing tumor of the central nervous system need to be considered in case of premature pseudopuberty.

\section{2}

Tumors in the cerebellopontine angle in children: Warning of a high probability of malignancy

Byung-Kyu Cho ${ }^{1,2}$, Ji Hoon Phi ${ }^{1}$, Kyu-Chang Wang ${ }^{1}$, In-One Kim ${ }^{3}$, Jung-Eun Cheon ${ }^{3}$, Jung Won Choi ${ }^{1}$, Seung-Ki Kim ${ }^{1}$
${ }^{1}$ Division of Pediatric Neurosurgery, Seoul National University Children's Hospital, Seoul, Republic of Korea; ${ }^{2}$ Department of Neurosurgery, The Armed Forces Capital Hospital, Gyeonggi-do, Republic of Korea; ${ }^{3}$ Department of Diagnostic Radiology, Seoul National University Children's Hospital

Background: Cerebellopontine angle (CPA) tumors are uncommon in children, and the pathological spectrum is different from that of adults. In this study, we reviewed the pathological diagnosis of pediatric patients with a CPA tumor to determine the pattern in this age group.

Methods: In a cohort of 267 patients with posterior fossa tumor, tumor locations were determined with preoperative magnetic resonance imaging (MRI). The pathological diagnosis, imaging characteristic, and treatment outcomes of patients with CPA tumors was reviewed and analyzed.

Results: Twenty-six patients $(9.7 \%)$ had a tumor in the CPA. The pathological spectrum was wide, from malignant intrinsic brain tumors to benign extra-axial tumors and sarcomatous lesions. Eighteen patients $(69 \%)$ had malignant tumors. The pathological nature was strongly linked to patient age. The mean age of malignant tumor group was significantly younger than that of benign tumor group. MRI findings that favored malignant histology included a plastic feature of the tumor, multiple signal voids, encasement of major arteries, widening of lateral recess, focal cerebellar edema, and hydrocephalus. The presence of seeding in the neuraxis also indicated malignant pathology. Especially, increased density on precontrast computed tomography was a strong predictor of malignant pathology. Malignant CPA tumors showed high surgical morbidity rate and grim long-term prognosis.

Conclusions: Patient age and tumor location are the two most important clues for the diagnosis of any brain tumor. Unlike in adult patients, clinicians should expect a high probability of malignant pathologies for pediatric CPA tumors, especially in infants and young children.

\section{3}

Reversible occlusion valve (On/Off) for intraventricular chemotherapy in shunt dependent brain tumor patients Christian Dorfer ${ }^{1}$, Andreas Peyrl ${ }^{2}$, Andrea Reinprecht ${ }^{1}$, Amedeo Azizi ${ }^{2}$, Monika Chocholous ${ }^{2}$, Irene Slavc ${ }^{2}$, Thomas Czech ${ }^{1}$

${ }^{1}$ Department of Neurosurgery, ${ }^{2}$ Department of Pediatrics and Adolescence Medicine, Medical University of Vienna, Austria

Introduction: In childhood malignant brain tumours intraventricular chemotherapy is part of current treatment strategies. In shunt-dependent patients a reversible occlusion On/Off shunt device to prevent drug clearance through the shunt was introduced by our group.

Methods: We retrospectively reviewed our pediatric brain tumour database for shunt dependent patients treated with intrathecal chemotherapy who had an On/Off device inserted in the shunt tubing in addition to a contralateral Ommaya reservoir. The technique has been previously reported (Czech et al., Ped Hemat Oncol 1997) The parents were familiarized with the occlusion mechanism.

Results: Between 1994 and 201217 children (m:f=12:5; mean age: 6.4 yrs, (range 1.5 to 15 ) had an On/Off device implanted. The diagnoses were medulloblastoma in eight, ependymoma in three, PNET in two, and GBM, plexuscarcinoma, ATRT, and infantile desmoplastic glioma, in one each. The insertion of the On/Off shunt and the reservoir were performed in separate procedures in thirteen patients and in one in four. No malfunction of the On/Off device occurred. In two patients the On/Off device was removed after the end of treatment in the setting of a valve revision. In one patient the On/Off-shunt was removed and converted to a $3^{\text {rd }}$ ventriculostomy. We encountered only one unintended occlusion of the On/Off device that was readily detected.

Conclusion: The On/Off device is a useful and safe adjunct in the management of shunt dependent children that require intrathecal chemotherapy. 
94

Brain tumours in infants: Experience with 38 cases

Dilip Dutta, Sandip Chatterjee

Park Clinic, Kolkata, India

Introduction: Brain tumours in infants form an unique group of tumours which convey a feeling of despair. It is to try and analyze our own experience of these rather rare tumours that we decided to review all our patients with brain tumours presenting below the age of one year. Material and methods: 38 patients were found with brain tumours in the period from 2000 to 2011. In 32, the tumour was supratentorial in location. The commonest presentations were increase in head circumference, irritability and failure to thrive. Unfortunately the majority of tumours had attained a fairly large size (greater than $5 \mathrm{~cm}$ in diameter) at presentation, presumably due to the compensatory capacity of the infant skull.

Results: The single largest group in the series were gliomas (15/38) out of which 12 were high-grade in nature. Teratomas $(5 / 38)$ and choroid plexus tumours $(6 / 38)$ were the next commonest in frequency. There were 4 infants with ATRT (atypical teratoid/rhabdoid teratomas), 3 with medulloblastomas, 2 with desmoplastic tumours of infancy, 2 with optic nerve tumours, and 1 with a craniopharyngioma. The prognosis was dismal with the high-grade gliomas as well as the ATRT tumours, in both cases all the babies were dead within 12-14 months of age. Of the two optic nerve tumours, one was operated upon, and one observed serially.

Conclusions: Tumours in infants represent a heterogenous group, not all of which have the same aggressive potential. Hence correct histological diagnosis is necessary to ascertain prognosis.

\section{5}

\section{Surgical experience in management of pineal region tumors} in Children's Cancer Hospital Egypt

Mohamed A. El Beltagy ${ }^{1}$, Mohamed Reda ${ }^{1}$, Alaa El Hadad ${ }^{2}$, Sameera Ezzat $^{3}$

${ }^{1}$ Neurosurgery Department, Children's Cancer Hospital Egypt (57357); ${ }^{2}$ Paediatric Oncology Department, Children's Cancer Hospital Egypt (57357); ${ }^{3}$ Research Department , Children's Cancer Hospital Egypt (57357)

Introduction: Optimal management decisions of pineal region tumors depend on the tumor pathology, germinomas are the most radio sensitive malignant tumors in central nervous system and can be cured without surgery, for benign pineal tumors complete resection is the procedure of choice and for malignant tumors radical resection is controversial.

Material and methods: 20 cases with pineal region tumors were operated upon in children's cancer hospital between 2008 and 2012. The operative decision, clinical outcome and complication were retrospectively reviewed to reach an ideal protocol for management.

Results: Out of 20 cases germ cell tumors represent 9 cases (45\%), pineoblastoma 8 cases (40\%), astrocytoma 2 cases $(10 \%)$ and one case of atypical rabdoid teratoid $(5 \%)$. patients age were ranging between 1.5 and 18 years with mean age 8.8 years. 13 cases were operated upon initially by open resection and gross total excision was achieved in 7 cases $(53 \%) .7$ cases were biopsied either endoscopic biopsy ( 5 cases) at time of endoscopic third ventriculostomy or stereotactic biopsy (2 cases). Accurate pathology was achieved in all biopsied cases. Following pathology results one case out of the biopsied required open resection aiming total excision (14.3\%). Cerebrospinal fluid diversion was performed in 19 cases, Endoscopic third ventriculostomy were done in all cases except 5 cases referred to us with ventriculoperitoneal shunt, out of the 14 cases of endoscopic third ventriculostomy 3 cases needed shunt later on with failure rate $21.4 \%$. Conclusion: Simultaneous endoscopic third ventriculostomy and biopsy is considered to be the ideal first step management for pineal region tumor with negative tumor marker. After which further management depend on pathology result.
96

The surgical aspects of cerebellar mutism syndrome in the pediatric age group

Mohamed A. El Beltagy ${ }^{1}$, Mostafa M. Atteya ${ }^{2}$

${ }^{1}$ Chief Neurosurgeon, Children's Cancer Hospital, Egypt; ${ }^{2}$ Assistant specialist of neurosurgery, Children's Cancer Hospital, Egypt

Introduction: Cerebellar mutism $(\mathrm{CM})$ is a unique syndrome that sometimes follows surgery of fourth ventricular tumors. Aim of the study is to discuss $\mathrm{CM}$ in cases operated through transvermian and telovelar approaches.

Methods: we reviewed the medical records of 80 children harboring fourth ventricular tumors treated at Children's Cancer HospitalEgypt (CCHE-57357) between 2008 and 2009. CM was assessed as regards onset, severity, duration, pattern of recovery, and tumor histopathology.

Results: CM occurred in 13 cases (16.2\%), 8 of which were medulloblastomas. Surgical approach was transvermian in 30 cases $(37.5 \%)$ and telovelar in 50 cases $(62.5 \%)$. Male to female ratio was 3:2. Mean age was 5.4 years ( $\mathrm{SD}=1.2$ years). In 30 cases operated through transvermian approach, CM occurred in 8 cases $(26.7 \%)$. Onset was acute postoperative in 7 cases $(87.5 \%)$, severe in 6 cases $(75 \%)$, of prolonged duration in 5 cases $(62.5 \%)$, and of partial recovery in 2 cases $(25 \%)$. On the other hand, in the 50 cases operated through telovelar approach, CM occurred in 5 cases $(10 \%)$, onset was 3-6 days post-operatively in 4 cases $(80 \%)$, and all of them were of the mild and transient type that resolved within 21 days $(100 \%)$. Recovery was complete in all 5 cases. Conclusions: The clinical pattern of $\mathrm{CM}$ is heterogeneous. It is more common, more severe, more acute, and of longer duration in the transvermian group than in the telovelar group. We initially worked through the transvermian approach then, we gradually shifted to telovelar approach which is our standard approach now.

\section{7}

Endoscopic assisted microsurgical resection of craniopharyngiomas in pediatric age group; Analysis and outcome of 50 cases Mohamed A. El Beltagy ${ }^{1}$, Mohamed R. Nagy ${ }^{2}$

${ }^{1}$ Assistant professor of neurosurgery, Kasr Al-Ainy School of Medicine, Cairo, Egypt, Chief Neurosurgeon, Children's Cancer Hospital Egypt; ${ }^{2}$ Lecturer of neurosurgery, Kasr Al-Ainy School of Medicine, Cairo, Egypt. Specialist of neurosurgery, Children's Cancer Hospital Egypt

Introduction: Simultaneous endoscopic and microsurgical resection is a novel way to approach craniopharyngiomas. Aim of the work is to discuss the usefulness of combined endoscopic and microsurgical approaches in treating craniopharyngiomas in pediatric age group. Methods: We retrospectively reviewed the clinical records and imaging data of 50 children harboring craniopharyngiomas treated at Children's Cancer Hospital-Egypt (CCHE-57357) between February 2008 and December 2011.

Results: The study included 50 patients with craniopharyngiomas. Female-to-male ratio was 2:3, with mean age of 7.6 years. Follow-up period 1-4.5 years. Pure microsurgical resection via pterional approach was used in 32 patients (64\%) while in 18 patients (36\%) endoscope was introduced for assisting microsurgical resection. Total removal of the tumor was achieved in 21 patients $(65.6 \%)$ in pure microsurgical resection group and 15 patients $(83.3 \%)$ in endoscopic assisted microsurgical group. 38 patients $(76 \%)$ required hormonal replacement therapy for diabetes insipidus; 27 patients $(84.4 \%)$ in pure microsurgical resection group and 11 patients $(61.1 \%)$ in endoscopic assisted microsurgical resection group. There were no mortalities in this series. There was no hypothalamic dysfunction in 29 patients $(90.6 \%)$ in pure microsurgical resection group and in all patients in endoscopic assisted microsurgical resection group. 
Conclusions: Endoscopic assisted microsurgical resection of craniopharyngiomas leads to higher rates of endocrine and hypothalamic function preservation with good clinical outcome while concurrently achieving comparable resections. It can be consolidated as effective and safe alternative for pure microsurgical resection in the treatment of these challenging tumors without adding any risk to the surgery.

\section{8}

Glioblastoma in childhood - Treatment and outcome of 9 patients Pedram Emami, Till Burkhardt, Jan Regelsberger, Manfred Westphal, Gertrude Kammler

Department of Neurosurgery, University Medical Center HamburgEppendorf

Introduction: While glioblastoma is very common in adults and is the most common primary malignant brain tumour in this age group, it remains a rare diagnosis in children. Postoperative treatment of Glioblastoma in adults is well standardized, but is under constant discussion in children and follows different types of study protocols (HITGBM A-D).

Methods: Treatment and outcome of 9 consecutive paediatric patients were retrospectively evaluated to determine the clinical course, median progression free survival and overall survival.

Results: 9 patients were diagnosed and treated between 2000 and 2013. The median age amounted to 9 years, all patients were younger than 16 . All but one of the patients underwent open surgery; complete resection was performed in 2 cases, subtotal resection in 6 cases. One patient underwent stereotactic biopsy due to the depth of the lesion. Combined radio-chemotherapy was performed in all cases, regarding the HIT-GBMC and -D and the HIT 2000 protocols as first-line therapy as well as Topotecan and Temozolomide as second line chemotherapy for recurrent disease. Median survival in this cohort was 16 months. Progression free survival was 3 months. Local tumour-recurrences were apparent in all cases. Median survival and progression-free survival in this small cohort did not correlate with extend of resection, depth of the lesion or the adjuvant regimen.

Conclusions: Survival of children with glioblastoma is being described as far better than in adults. Despite following the common study protocols, we cannot confirm these results in this cohort, most probably due to the low case number.

\section{9}

\section{The role of open surgery in treatment of pineal lesions} in the paediatric age group

Pedram Emami, Gertrud Kammler, Manfred Westphal

Department of Neurological Surgery, University Medical Center Hamburg-Eppendorf, Hamburg, Germany

Objective: Pineal region lesions are rare and diverse entities requiring therapy regimens depending on acute clinical symptoms and diagnostic findings, especially the histological diagnosis. Therefore gaining a sufficient and representative tissue sample remains one of the main goals of the operative procedure, so we focused on the latter as an essential part of the therapeutic strategy.

Patients and Methods: 47 persons younger than 18 years underwent 50 operative procedures in our institution from 1981 to 2012. We analyzed the initial clinical and tumour markers findings, operative techniques and complications, the treatment of hydrocephalus and the outcome retrospectively.

Results: 40 craniotomies (35 total, 5 subtotal resections), 7 stereotactic and 3 endoscopic biopsies were performed. 20 germ cell tumours, 15 pineal parenchymal tumours, 6 gliomas, 2 pineal cysts and 4 other entities were found. Markers were determined routinely in serum and also in CSF in some cases. Negative tumour markers couldn't exclude the presence of a germ cell tumour. The most common symptom was hydrocephalus, which was reversible after surgery. 3 cases of perioperative complication occurred during open surgery, none led to any neurological deficit. No complications were associated to the stereotactic or the endoscopic biopsies, but none of the latter led to a histological diagnosis.

Conclusion: Open surgery is not only an effective method to achieve a complete tumour removal, it may also be the more useful method to both treat the occlusive hydrocephalus and to come to a definite and reliable diagnosis with comparatively low risk of periopertive complications and favourable outcome.

\section{0}

Correlation between the clinical evolution and the histological subtype of medulloblastomas in a pediatric series operated between 2000 and 2013

Blandine Grassiot ${ }^{1}$, Pierre-Aurélien Beuriat ${ }^{1}$, Alexandru Szathmari ${ }^{1,4}$, C. Rousselle ${ }^{1}$, Didier Frappaz ${ }^{2}$, L. Claude ${ }^{2}$, A. Vasiljevic ${ }^{3,4}$, A. Jouvet ${ }^{3,4}$, Carmine Mottolese $\mathrm{e}^{1}$

${ }^{1}$ Service de Neurochirurgie pédiatrique E, Hôpital Pierre Wertheimer, Groupement hospitalier EST, Hospices civils de Lyon, Bron, France;

${ }^{2}$ Institut d'Hématologie et d'Oncologie Pédiatrique, Centre Léon Bérard, Lyon; ${ }^{3}$ Service de Pathologie et de Neuropathologie, Centre de Biologie et Pathologie Est, Groupement Hospitalier Est, Hospices Civils de Lyon, France; ${ }^{4}$ Centre de Recherche en Neurosciences de Lyon, INSERM U1028, CNRS UMR5292, Equipe Neuro-Oncologie et Neuro-Inflammation, Lyon, France

Introduction: The multidisciplinary approach of the treatment of the medulloblastomas improved the outcome of these children in last years. The prognostic is also related to the histologic subtype but the role of the histologic markers is not well defined. We studied the relationship between the histologic subtypes of medulloblastomas and the clinical evolution.

Methods: Between 2000 and 2012, 42 cases of medulloblastomas were treated in our service. The mean age was 7.9 years. All the children were treated by surgery (34/42 complete removal) and radiotherapy and/or chemotherapy according to the extension of the disease. Following the WHO classification, we distinguished three histologic subtypes: classical, desmoplasic and anaplastic. Each type was correlated with the clinical good or bad evolution (recurrence or death).

Results: Among the 42 cases of medulloblastomas, 32 were classical (10 metastatic at time of diagnosis), 7 were desmoplasic ( 2 metastatic) and 3 anaplastic (3 metastatic). The progression free survival (PFS) was respectively for each type, $78 \%, 57 \%$ and $60 \%$ with an equivalent treatment for all groups.

Discussion: Our study shows that the classical medulloblastomas have a better PFS. It confirms that the prognostic change with the histological type. It is necessary to follow this research in order to find histopathologic factors and permit to improve the prognostic, modulate the complementary treatment and then limit our sequels. It was also interested to add the molecular biology that permits to obtain new markers very useful for a new $t$ classification of medulloblastomas.

\section{1}

Split bone repair after complete resection of an unusual supraorbital manifestation of a desmoid tumor invading the sagittal sinus in a 19-months-old girl

Christian Hagemann, Philip O. S. Kunkel

Department of Pediatric Neurosurgery, Altona Children's Hospital, Hamburg, Germany

Introduction: Following minor head trauma a 14 months old girl developed progressive swelling on the forehead. Neurosurgical consultation 4 months later revealed a solid supraorbital lesion of $4 \times 5 \mathrm{~cm}$. Ultrasound and X-ray studies displayed signs of malignancy. CT- and MRI scans 
showed a contrast enhancing osteoclastic lesion invading dura mater and superior sagittal venous sinus. Biopsy revealed a desmoid tumor (aggressive fibromatosis). These rare soft tissue neoplasms predominantly arise in the extremities, abdominal cavity or thorax. Some cases of desmoid tumors originating in the skull base and mandible have been reported. Gross total resection represents the main treatment modality. In recurrent or unresectable cases a variety of adjuvant therapies are used.

Methods: In this case gross total resection was achieved via bicoronal incision and supraorbital frontal craniectomy followed by resection of invaded dura and sagittal venous sinus. Histology confirmed tumor free margins. After patch repair of the dura the osseous defect was closed in split bone technique harvesting bone from the right parietal region. Divided bone parts were attached using resorbable plates and screws.

Results: At 3, 6 and 12 months follow-up no signs of bone osteolysis or tumor recurrence in MRI studies could be detected.

Conclusions: Review of the literature yielded only two other cases of desmoid tumors arising from calvarial bone of pediatric patients, one associated with Gardner's syndrome. We here report a case were margin free desmoid tumor resection was possible. Simultaneously excellent cosmetic outcome could be accomplished applying a split bone technique in a young child.

102

The typicality of atypical pediatric meningiomas

Yasser Jeelani, Grace Young, Stephanie Da Silva, J. Gordon McComb, Mark Krieger

Division of Neurosurgery, Children's Hospital Los Angeles, Department of Neurological Surgery, Keck School of Medicine University of Southern California, Los Angeles, CA, Unites States of America

Introduction: Meningiomas, a rare entity in children, are conventionally graded based on their predilection towards aggressive or malignant behavior. Atypical Meningiomas (WHO II) form an intermediate group between typical (WHO I) and anaplastic (WHO III) forms, and are thought to have a tendency to be locally aggressive and recurrent, besides being more frequently associated with neurofibromatosis type 2 (NF-2). Methods: Under an IRB approved study, a retrospective review was performed on all such children who underwent primary surgical resection between $2002 \& 2012$.

Results: A total of 20 patients (12 male) were identified; 13 intracranial, 6 spine and 1 involving both. After primary surgical resection, 14 were pathologically identified as typical \& 6 as atypical. $5 / 14$ of the typical cases had a history of radiation exposure for a separate brain tumor, while the same was true for only $1 / 6$ of the atypical cases $(\mathrm{p}=0.61) .6$ cases with a prior history of NF-2 were all associated with typical meningiomas, with no history of NF-2 found in any of the atypical cases $(p=0.11) .8$ recurrences were observed after a mean follow-up of 4 years. While 2 occurred in patients that underwent only subtotal resection, the remaining 6 occurred in patients with radiologically determined gross-total tumor resection. Histologically, 5 of the total of 8 recurrences occurred in typical cases, with 3 occurring in atypical cases $(\mathrm{p}=0.64)$.

Conclusions: Pediatric meningiomas histologically classified as atypical do not seem to occur more frequently in children with a history of radiation exposure or in children with NF-2. The recurrence rates are also not significantly between atypical \& typical meningiomas.

\section{3}

A heterogeneous group, brain tumors in the first year of life a report of 25 patients in our institution

Gertrud Kammler ${ }^{1}$, Uwe Kordes ${ }^{2}$, Christian Hagel ${ }^{3}$, Jens Fiehler ${ }^{4}$, Manfred Westphal ${ }^{1}$

Departments of ${ }^{1}$ Neurosurgery, ${ }^{2}$ Paediatric Hematology and Oncology, ${ }^{3}$ Neuropathology, ${ }^{4}$ Diagnostic and Interventional Neuroradiology, University Medical Center Hamburg Eppendorf, Germany
Introduction: Children younger than 12 months of age represent $2 \%$ of all pediatric brain tumours and still have poor prognosis. The purpose of this description was to analyze the specific clinical features, diagnosis and survival in terms of treatment.

Methods: This study analyzed retrospectively 25 infants with brain tumours treated in our institution between 1998 and 2012. Their clinical records were examined concerning age at diagnosis, clinical symptoms, MRI-studies, surgical procedures, histology and adjuvant therapy.

Results: 25 infants, median age of 4 months were diagnosed. The main clinical feature was macrocephalus in up to $70 \%$. Focal neurological symptoms were seen in 5 patients, four patients were diagnosed prenatally. 24 patients underwent surgery. Histology showed 36\% (9) low grade, while $64 \%$ (16) were malignant tumours. The location favors the supratentorial compartment. Resection was complete in 8 , subtotal in 7 and partial in 3 patients. Three patients underwent biopsy. One was diagnosed by CSF, one patient was refused from diagnosis. One patient died perioperatively.

Conclusions: Tumours of the central nervous system in infants are rare and show a great spectrum of histological types. As pediatric braintumors are usually in the infratentorial compartment, the very early tumors of newborns seem to be derived from a different biological background. Most of the tumours have a massive size so that prognosis is still poor. The detection of congenital brain tumour does not preclude normal development but warrants early intervention. Given the wide spectrum of possible histologies, a considerable early multidisciplinary approach assures optimal outcome.

\section{4}

\section{Brainstem in surgery for cerebral tumors}

Villiam A. Khachatryan ${ }^{1}$, Vitaly A. Khilko ${ }^{2}$, Alexander V. Kim ${ }^{1}$

${ }^{1}$ Russian Polenov Neurosurgical Institute, Saint-Petersburg, Russia; ${ }^{2}$ Neurosurgical Department of City hospital \#2, Saint-Petersburg, Russia Aim: to elaborate an algorithm evaluating brainstem tumor involvement to choose surgical strategy.

Materials and methods: Results of examination and surgical treatment of 1005 patients with posterior fossa tumors of various site and histopathology, aged 1 to 72 , followed up for 3 to 20 years, were analyzed.

Results: Neuroectodermal tumors $-3 / 4$, grade II-IV $-2 / 3$. All brainstem tumor cases were divided into primary (intrinsic, exophytic) tumors (176 cases), secondary (181), parabrainstem (220), stem-deforming (238), and tumors not connected with the brain stem (190). Total or subtotal removal was carried out in $82 \%$ of the cases. Postoperative mortality (for 3 months) made $2.3 \%$. Retrospective analysis revealed the results to depend on degree of tumor removal, the latter depending on brainstem involvement by tumor. Different brainstem areas were manipulated on in 484 procedures of tumor removal. The analysis of postoperative course denotes that in vital brainstem areas, i.e. paraaqueductal grey matter, macula cerulea, hypoglossal and vagal triangles, involvement manipulations aimed at total tumor removal result in vital functional disturbances and thus are inexpedient. If functionally important brainstem areas, i.e. acoustic and vestibular fields, superior and inferior quadrigeminal tubercles, pyramids are involved, total tumor removal is accompanied by stable neurologic deficit and can be performed if additionally indicated only. In supra- and subcollicular triangle, raphe area, funicular bodies involvement, total tumor removal is quite expedient. Functional role of other brainstem areas is being precisely evaluated.

\section{5}

Outcome analysis of childhood supratentorial astrocytomas: a retrospective study of 33 repeated cases at a single institution Alexander V. Kim, Villiam A. Khachatryan

Russian Polenov Neurosurgical Institute, Saint-Petersburg, Russia

This research is based on the retrospective analysis of the records of the Russian Polenov Neurosurgical Institute between 2000 and 2012. 160 
patients aged 2 months to 17 years underwent surgery for supratentorial astrocytomas during this period. $33(20.6 \%)$ of them are operated repeatedly because of tumor recurrence. The boys/girls ratio was 1.06 .

Results: Primary (after first operation) tumors included low-grade astrocytoma - $102(63.7 \%)$, anaplastic astrocytoma - 39 (24.5\%), glioblastoma $-19(11.8 \%)$. Among 33 patients who were operated repeatedly grade I-II astrocytomas were in $14(42.4 \%)$ cases, anaplastic astrocytomas - 11 $(33.3 \%)$, glioblastomas $-8(24.3 \%)$. There were 6 cases of anaplastic transformation of low-grade astrocytomas to high-grade astrocytomas. The tumors of middle localization were in 15 (45.5\%) cases, involving 2 or more cerebral structures in $11(33.3 \%)$. Primary removal was total or subtotal in $145(90.6 \%)$ cases, partial in $15(9.4 \%)$. In the series under study total or subtotal tumor removal was achieved in 27 cases of 33 $(81.8 \%)$, partial $6(18.2 \%)$. The operations were repeated in 3 months to 12 years. The results of surgical treatment of supratentorial astrocytomas are discussed, depending on the age and preoperative state of the patient, character of tumor (grade, type of grow, etc), surgical approach and totality, staging, and type of adjuvant therapy, in order to understand pathogenesis of the repeated tumor development, the factors influencing the duration of progression-free period, criteria of the efficiency of repeated operations. An algorithm of complex treatment of recurrence patients of the given neoplastic group is planned to elaborate.

106

The efficacy of temozolomide in the terminal stage of medulloblastoma patients and their tumor biology

Akihide Kondo, Mario Suzuki, Osamu Akiyama, Ikuko Ogino, Kazuaki Shimoji, Masakzu Miyajima, Hajime Arai

Department of Neurosurgery, Juntendo University School of Medicine, Tokyo, Japan

Introduction: Medulloblastoma is a difficult pediatric tumor to cure. Occasionally, patients have to give up aggressive treatment and selected maintenance therapy such as oral chemotherapy using etoposide or temozolomide for some reason. At that point, it is important to select appropriate agents based on tumor biology individually. The fact is well known that the DNA methylation status of the $\mathrm{O}_{6}$-methylguanine methyltransferase (MGMT) gene increase the susceptibility of tumors to alkylating chemotherapeutic agents. Then, we studied the relationship between the methylation status of MGMT in primary medulloblastomas and the period of stable disease in our patients treated with temozolomide as their maintenance therapy.

Methods: We reviewed the period of stable disease in the patients treated with temozolomide as an agent for their maintenance therapy. We also studied the methylation status of MGMT in their tumors using immunohistochemical stainings and whole wide sequence technics with widely utilized methods. Results: There are no major complications in the terminal stage of medulloblastoma patients who used temozolmide as their maintenance therapy. The number was too small to show the statistically significant meaning that temozolimide prolonged the period of stable disease in these patients between the differences of methylation status of MGMT.

Conclusion: Further studies are needed to show that the methylation status of MGMT is the key for the selection of agents in the maintenance therapy of medulloblastoma. Temozolomide may be the one of the good candidate for the maintenance therapy for medullobalstoma because of its safety.

\section{7}

The use of intraoperative CT navigation and resection control in pituitary surgery promises a better intraoperative orientation and resection control in distinct pediatric indications

Stefan Linsler, Sebastian Antes, Joachim Oertel

Department of Neurosurgery, Saarland University, Homburg/Saar, Germany

Object: The safety of endoscopic skull base surgery can be enhanced by accurate navigation in preoperative CT and MRI. Here we report our initial experience of real-time intraoperative computed tomographyguided navigation surgery for pituitary tumors in childhood.

Methods: We report the case of a 15 -year-old girl with a huge STH-secreting pituitary adenoma with supra- and perisellar extension. Also the skull base was infiltrated. In this case we performed a transnasal transsphenoidal approach for debulking the adenoma and for optic chiasm decompression. We used a MRI neuronavigation (Medtronic Stealth Air System) which was registered via intraoperative CT scan (Siemens CT suite). Preexisting MR imaging studies (navigation protocol) were fused with the intraoperative CT scans to enable 3D navigation based on MR and CT imaging data. Intraoperative we did a further $\mathrm{CT}$ scan for resection control.

Results: The intraoperative accuracy of the Neuronavigation was excellent. There was an optimal accuracy with an adjustment of less than $1 \mathrm{~mm}$. The navigation was very helpful for orientation in the destroyed skull base, particularly within the sinus sphenoidalis and parasellar space. After opening the sellar region and tumor debulking we did a CT scan for resection control because the extent of resection was not credible evaluable in this huge infiltrating adenoma. Thereby we were able to demonstrate a sufficient decompression of the chiasma and complete resection of the medial part of the adenoma in the intraoperative CT images.

Conclusions: The use of intraoperative CT/MR imaging-guided neuronavigation for transsphenoidal surgery is a time-effective, safe, and technically beneficial technique.

\section{8}

Nasoethmoidal meningioma with cytogenetic features of tumor aggressiveness in a 16-year-old child: a case report followed up over 11 years

Stefan Linsler, Joachim Oertel, Stefanie Urbschat, Ralf Ketter

Department of Neurosurgery, Saarland University, Homburg/Saar, Germany

The case of a 16-year-old girl is presented who was admitted to our hospital because of severe frontal headache in January 2002. Magnetic resonance scans revealed a nasoethmoidal tumor on the right side, with erosion of the skull base and invasion of the right orbit. A needle biopsy specimen revealed the histological diagnosis of a fibroblastic meningioma (WHO grade II), with histochemically determined partial activity loss of alkaline phosphatase. The tumor was completely resected by a combined intracranial-transbasal and transnasal approach. In this case, we found a meningioma with deletion of the short arm of chromosome 1 through a translocation between chromosomes 1 and $11[\mathrm{t}(1 ; 11)]$ as well as additional chromosomal aberrations, including partial or complete monosomy of chromosomes 2, 6, 7, 11, 13 and 22, a cytogenetic pattern known to be associated with elevated tumor aggressiveness in meningiomas of adult patients. Accordingly, this juvenile meningioma may biologically correspond to a WHO grade II adult-type meningioma with an increased risk of recurrence. Therefore, we incorporated this patient into an intensified schedule of postoperative care. The clinical course of the child until today, which was determined by two further resection due to tumor recurrence as well as radiotherapy is presented.

\section{9}

Non-surgical management of atlantoaxial Langerhans cell histiocytosis. A case report

Long Ai Sha, David Low Chyi Yeu, Seow Wan Tew

Neurosurgical Service, Department of Pediatric Surgery, KK Womens and Children's Hospital, Singapore

Introduction: The treatment protocol for cervical langerhans cell histiocytosis $(\mathrm{LCH})$ is highly varied. Treatment modalities run the gamut from medical (e.g. immobilization, steroid, chemotherapy, radiotherapy) to surgery (biopsy, curettage, excision of tumor, fusion with instrumented fixation). Management largely bases on the consideration of clinical symptoms, neurological involvement, and bony stability. 
Results: We report a 6-year-old Chinese girl with histologically confirmed unifocal, monostotic Langerhans cell histiocytosis of $\mathrm{C} 1 / 2$. She presented with 1 month history of left neck pain with torticollis associated with bilateral cervical lymphadenopathy. No neurological deficit was elicited clinically. CT and MRI demonstrated fixed rotatory subluxation of $\mathrm{C} 1$; soft tissue erosion of clivus, odontoid process and anterior arch of atlas. The destructive lesion is extending into the right prepontine cistern. Radiologic-guided biopsy of right cervical lymph node confirmed CD1positive LCH. Bone marrow aspiration ruled out marrow involvement. Radionuclear bone scan was consistent with the unifocality of the disease. Halo frame fixation was applied. She was treated with prednisolone and vinblastine. Surveillance imaging showed stable disease with no significant reduction in the extent and size. Suboptimal response after a 3months' trial of vinblastine prompted initiation of cladribine. Subsequent imagings reveal reossification of the initial lytic lesions. Upon removal of halo frame at 10 month, there is no instability clinically and radiologically. She remained in remission upon completion of 7 cycles of cladribine. Conclusion: Symptomatic cervical LCH without myelopathy can be managed non-surgically with good short-term response.

\section{0}

Pediatric high-grade gliomas: a clinicopathological report of 28 cases Chenkai Ma, Yang Zhao, Xiaoguang He, Jie Ma

Department of Pediatric Neurosurgery, Xinhua Hospital, Shanghai Jiao Tong University School of Medicine

Introduction: High-grade gliomas are relatively uncommon central nervous system tumors in children, accounting for $8-10 \%$ of all pediatric brain tumors. Hence, the prognostic factors of pediatric patients with HGG remain unclear. To determine the favourable factors of survivals, we reviewed retrospectively clinicopathological features and outcomes of children treated for HGG in our center.

Methods: Twenty-eight patients diagnosed as HGG (including glioblastoma) from 2006 to 2012 were included this study. Clinical and pathological features as well as outcome of these patients were collected and evaluated. Statistically survival analysis and Cox regression was conducted by SAS. Results: The mean age of the included patients was 5.38 \pm 3.76 years. Sex ratio (male:female) is 4:3. Radiologically HGG were presented as uneven-enhanced. The tumors diagnosed as WHO III and IV stages were 13 and 15 , respectively, 15 of which were glioblastomas. The positive of $\mathrm{P} 53$ is $55.5 \%$. Sex, age, stage and $\mathrm{P} 53(\mathrm{P}=0.6514,0.7317,0.8877$ and 0.7411 , respectively) are not the risk factor of the survival of HGG. Conclusions: High-grade gliomas are rare malignant brain tumor in pediatric patients. Clinicopathological features fail to uncover the prognosis of the patients. To prolong the survival, adjuvant therapy should be taken.

\section{1}

Use of apparent diffusion coefficient mapping in medulloblastomas Neena I. Marupudi ${ }^{1}$, Deniz Altinok ${ }^{2}$, Luis Goncalves ${ }^{2}$, Dhanya Anand ${ }^{1}$, Steven D. Ham ${ }^{1}$, Sandeep Sood ${ }^{1}$

${ }^{1}$ Department of Neurosurgery and Pediatric Neurosurgery, ${ }^{2}$ Department of Pediatric Neuroradiology, Wayne State University School of Medicine and Children's Hospital of Michigan, Detroit, Michigan, United States

Introduction: Although conventional MRI is essential in evaluation of pediatric posterior fossa tumors, it still offers limited information regarding extent of tumor infiltration of adjacent critical structures. Because medulloblastomas demonstrate diffusion restriction on apparent diffusion coefficient map sequences (ADCms), we investigated whether ADCms predict brain stem and cerebellar peduncle infiltration by medulloblastomas, potentially defining surgical planes.

Methods: A retrospective review of patients undergoing surgical resection of pathologically confirmed medulloblastomas. Experienced pediatric neuroradiologist reviewed the MRIs/ADCms, assessing planes between tumor and cerebellar peduncles/brainstem. Independent evaluator documented surgical findings from operative reports for comparison to radiographic findings. Sensitivity, specificity, PPV, and NPV of the ADC maps to predict involvement of the right, middle, and left brainstem, and right and left cerebellar peduncles was calculated.

Results: 32 patients with medulloblastomas underwent surgical resection at our facility (2004-2012); 25 patients met inclusion criteria. Mean age at surgery was $8.2 \pm 4.2$ years. PPV of ADCms to predict tumor invasion of brainstem and cerebellar peduncles ranged from $61.5 \%$ to $100 \%$; NPV ranged from $66.7-88.9 \%$. Sensitivity approached $80 \%$ while specificity approached $84 \%$. ROC-area ranged from 0.73 at cerebellar peduncles to 0.79 at the brainstem.

Conclusion: ADCms are valuable in predicting the infiltrative/noninfiltrative planes along the tumor-brainstem interface in medulloblastomas. Inclusion and evaluation of ADCms in preoperative evaluation may play a role in surgical resection planning, influencing the surgeon's technical approach and how extensively the neurosurgeon may pursue a complete resection.

\section{2}

Disseminated oligodendrioglial-like leptomeningeal tumor in a child - Case report

Viviana Minichini ${ }^{1,2}$, Michal Rynkowski ${ }^{1}$, Henri Steyaert ${ }^{2}$, Isabelle Salmon ${ }^{3}$, Oliver De Witte ${ }^{1}$

${ }^{1}$ Department of Neurosurgery, Erasme Hospital, Universite Libre de Bruxelles (ULB), Brussels, Belgium; ${ }^{2}$ Department of Pediatric Surgery, Hopital Universitaire des Enfants Reine Fabiola (HUDRF), Brussels, Belgium; ${ }^{3}$ Department of Pathology, Erasme Hospital, Universite Libre de Bruxelles (ULB), Brussels, Belgium

Introduction: Disseminated oligodendroglial-like leptomeningeal tumors are very rare pediatric neoplasms of the CNS, which are commonly associated with cystic or nodular T2 hyperintense lesions usually located in the posterior fossa, basal brain region and/or the spinal cord accompanied by hydrocephalus. However, diffused leptomeningeal lesions may mimic tuberculous meningitis, neurosarcoidosis, vasculitis, secondary gliomatosis and unusual infectious meningitis in children, which should be considered in the differential diagnosis. Herewith, we present a very rare case of a child with a disseminated oligodendroglial-like leptomeningeal tumor with a differential diagnosis protocol.

Material and Methods: A 3-year-old girl was referred to our institution with a 2-month history of vomiting, headache and apathy. Brain and total spine MRI revealed hydrocephalus, diffuse leptomeningeal thickening of the basal brain region and in the posterior fossa, as well as leptomeningeal enhancement at the thoracic level. CSF analysis revealed no neoplastic cells and no evidence of CNS infection. A posterior fossa craniotomy with a cerebellar biopsy was performed, followed by VP shunt placement. Immunohistochemistry demonstrated strong reactivity for OLIG2 and synaptophysin. Proliferation index KI-67 was 10\%. FISH test demonstrated 1p loss/19q intact. Detailed histopathologic examination revealed disseminated oligodendroglial-like leptomeningeal tumor.

Conclusions: Disseminated oligodendroglial-like leptomeningeal tumors represent a pathological entity. Although rare, these tumors should be considered in a case of idiopathic meningitis, without any signs of primary brain or spinal cord neoplasm. Therefore, a surgical meningeal biopsy of contrast enhancing area should be considered as a diagnostic tool.

\section{3}

Infrantentorial ganglioglioma in a child - Case report

Viviana Minichini $^{1,2}$, Michal Rynkowski ${ }^{1}$, Isabelle Salmon ${ }^{3}$, Oliver De Witte ${ }^{1}$

${ }^{1}$ Department of Neurosurgery, Erasme Hospital, Universite Libre de Bruxelles (ULB), Brussels, Belgium; ${ }^{2}$ Department of Pediatric Surgery, Hopital Universitaire des Enfants Reine Fabiola (HUDRF), Brussels, Belgium; ${ }^{3}$ Department of Pathology, Erasme Hospital, Universite Libre de Bruxelles (ULB), Brussels, Belgium 
Introduction: Ganglioglioma is a very rare primary neoplasm comprising up to $10 \%$ of primary pediatric CNS neoplasms. Posterior fossa gangliogliomas represent less than $15 \%$ of these cases. Gross-total resection in the infratentorial region is often not possible and is associated with important morbidity. Regardless tumor's location the postoperative adjuvant therapy following subtotal resection of gangliogliomas is still controversial. Herewith, we present a case of a posterior fossa low-grade ganglioglioma with an unfavorable outcome along with a brief review of current literature on operative and adjuvant therapy.

Material and Methods: A 9-month-old infant was admitted with a large posterior fossa tumor and marked hydrocephalus. The patient was operated on twice with partial and gross total resection, respectively. The radiological follow-up including MRI and PET-scan following both operations was very unfavorable with a vast progression of a lesion. PET-scan demonstrated hypermetabolism. Pathological examination following both operations revealed a ganglioglioma (WHO Grade I) brain tumor with an index of proliferation less than 1\% (anti-KI67). The postoperative course was marked with extremely high progression of a lesion and a clinical deterioration. The patient died two weeks after the second operation. Conclusions: Ganglioglioma is an uncommon neoplasm of the CNS that rarely presents in the posterior fossa. Even though, in the majority of cases the course of the disease is benign, it may present very unfavorable outcome as in the presented case. The adjuvant therapy following subtotal resection is still controversial and there is certainly a need for further randomized studies in this field.

\section{4}

\section{Surgical indication and natural course of intracranial infantile myofibromatosis}

Tomoru Miwa ${ }^{1,2}$, Yuichiro Nonaka ${ }^{2}$, Shizuo $\mathrm{Oi}^{2}$, Hikaru Sasaki ${ }^{1}$, Kazunari Yoshida ${ }^{1}$

${ }^{1}$ Department of Neurosurgery, Keio University School of Medicine, Tokyo, Japan; ${ }^{2}$ Department of Neurosurgery, The Jikei University School of Medicine, Tokyo, Japan

Introduction: Infantile myofibromatosis (IF) are the most common fibrous tumor of infancy and early childhood. However, intracranial myofibromatosis cases are very rare. The lesions are said usually arise from the dura or sometimes intraparenchymal.

Methods: We report our unique one-month-old male infant case of intracranial IF, and then we reviewed the surgical indication and natural course of intracranial IF by literatures.

Results: In our case, intracranial IF showed spontaneous regression occurred at the same time as that of a lumbar lesion 3 months after biopsy, and magnetic resonance venography demonstrated the recanalization of transverse sinus due to the tumor regression. Among previous 17 cases of intracranial IF in the literature, 11 cases were performed total or subtotal removal and only 2 cases showed recurrence. Four of 5 cases with biopsy and observation (one patient died due to cardiac complications) exhibited spontaneous regression with no recurrence and the term was ranged from 7 months to 4 years (average 22 months).

Conclusion: Spontaneous regression is a characteristic of intracranial IF without visceral involvement. Although, diagnosis by biopsy is indispensable for intracranial IF, even if the tumor is very large and compresses the brain, or sinuses were occluded with no symptom, it is not necessary to remove it quickly. Because spontaneous regression can be expected, the first choice of management is close observation. Even if a tumor appears aggressive, management should not be performed in the same way as that for other intracranial tumors.

\section{5}

Profile of pediatric brain tumors at a tertiary care neuro-oncology ventre - Patterns of care and perioperative outcomes

Aliasgar V. Moiyadi, Prakash M. Shetty

Department of Neurosurgery, Tata Memorial Centre, Mumbai, India
Introduction: Management of pediatric brain tumors is challenging requiring multidisciplinary care. Optimization of surgery and perioperative care is essential. We describe our experience at a tertiary-care, referral, dedicated neuro-oncology setup in a resource-constrained setting.

Methods: 187 consecutive pediatric tumors ( $<18 \mathrm{yrs})$ operated between 20072012 were reviewed from a prospective database. Demographic, clinicoradiological, pathological and perioperative outcomes were analysed. Results: The commonest presenting features were raised ICP (58\%), neurological deficits $(50 \%)$ and seizures $(20 \%) .50 \%$ had undergone prior treatment, including 51 children who had undergone only a prior surgery and were re-operated at our centre for "completion" surgery. Histologically, glial tumors were the commonest (40\%), followed by embryonal $(23 \%)$ and craniopharyngiomas ( $8 \%$ ). "Completion" redo-surgery was more often performed in embryonal tumors (40\%), and craniopharyngiomas (40\%), than gliomas (30\%). Astrocytomas and ependymomas occurred more frequently in the supratentorial compartment, embryonal tumors (PNETs) were commoner in the posterior fossa. $60 \%$ were large at presentation ( $>4 \mathrm{~cm}$ ). Radical resections were achieved in $69 \%$. Major neurological morbidity was $13 \%$. Perioperative mortality rate was $7.5 \%$. This was significantly higher in the emergent surgeries $(23.3 \%$ vs $4.5 \%)[p=0.002]$. There was no difference in morbidity between first and redo surgeries.

Conclusions: Because of referral patterns we see a high percentage of redo surgeries. Optimizing surgery upfront (extent of resection as well as perioperative outcomes) is important to avoid unnecessary reoperations. Even when reoperation is required, morbidity is not prohibitive and with careful case selection, it is possible to get good outcomes.

\section{6}

Neurosurgical procedures in the semi-sitting position in children Guilherme R. Montibeller, Christoph A. Tschan, Joachim Oertel Department of Neurosurgery, Saarland University, Medical Center and Saarland University, Faculty of Medicine, Homburg/Saar, Germany

Objective: The semi-sitting position is a controversial issue in neurosurgery. Several authors consider this position dangerous, mainly because of the risk of venous air embolism. It offers several advantages, especially during surgeries in the posterior cranial fossa and cerebellopontine angle. In the pediatric population with frequent pathologies of the posterior cranial fossa it is an important possibility of patient positioning. The aim of this study is to observe the relevance of this technique during treatment of different pathologies in this area.

Methods: Between March 2011 and August 2012, 10 pediatric Patients were operated on pathologies in the posterior cranial fossa. All surgeries were performed in semi-sitting position by the senior author. Surgical reports, diagnostic imaging and intraoperative video-recordings were analysed. The possibility of recognition of anatomical structures, safety, as well as possible advantages of the technique was evaluated.

Results: The mean age of the operated children was of 10-years-old (range 5-17). Flush of blood and irrigation fluid out of the operative field through gravity allowed early anatomical structures recognition and the performance of bimanual microsurgery in all cases. No venous air embolism or other complications related to the positioning of the patients were observed. Bleeding during surgery was reduced.

Conclusions: The semi-sitting positioning of patients in pediatric age seems to be safe and provides during microsurgery in the posterior cranial fossa considerable advantages in some demanding situations. The reduced bleeding is important in this group of patients and could be observed in this series. The additional time required positioning the patient might shorten the operation time because of the facilitated dissection condition.

\section{7}

Secondary brain tumors after cranial irradiation in childhood

Yuta Murakami, Jun Sakuma, Yuka Matsumoto, Keiko Oda, Yusuke Kuromi, Masayuki Yamada, Masahiro Ichikawa, Takamitsu Tamura, Yugo Kishida, Taku Sato, Kiyoshi Saito 
Department of Neurosurgery, Fukushima Medical University, Fukushima, Japan

Cranial irradiation has been used to treat brain tumors or for central nervous system (CNS) prophylaxis of acute lymphoblastic leukemia (ALL). While the prognosis of brain tumors or acute childhood leukemia has improved, long-term survivors have an increased risk of developing CNS tumors, such as gliomas, meningiomas, or angiomas. We report 6 patients with secondary brain tumors after childhood cranial irradiation. Five patients received 18 or 24 Gy of cranial irradiation for ALL at their ages of 3 to 11 years old. After 8 to 18 years, they were suffered from cavernous angioma in 2, atypical meningioma in 3, and meningioma in 1. Another patient who received 40 Gy of irradiation at age 4 developed meningioma 21 years later. Except for one cavernous angioma, 5 tumors were surgically removed. Three cases with atypical meningioma were especially difficult to treat due to an aggressive and invasive biology of the tumors. Therefore we recommend systematic cranial imaging and long follow-up of survivors, who received cranial irradiation, to detect brain tumors before they progress.

\section{8}

Diagnosis and surgical treatment of brain tumors supratentorial localisation in children

Dilorom Murodova $^{1}$, Mahmud Ahmediev ${ }^{2}$

${ }^{1}$ Neurooncology, ${ }^{2}$ Pediatric Neurosurgery, Republican Scientific Center of Neurosurgery, Tashkent, Uzbekistan

One of the main trends of modern neurosurgery, is to minimize surgical trauma and improve the quality of life of patients after surgery.

The aim of the study: Comparative evaluation of the results of surgical treatment of brain tumors method using DT-MRI tractography.

Materials and methods: We analyzed the results of treatment of 42 patients who were operated under identical conditions. Surgery was performed under the control of intraoperative monitoring. For noninvasive and more complete removal of the tumor with maximum preservation of pathways using data mechanogram, which allows the surgeon to more accurately determine the amount of tumor resection. This technique made it possible to identify possible ways to access and surgical planning of tumor resection.

Results and discussion: These DT-MRI tractography, found that nearly half $(58.5 \%)$ of primary surgery ended with subtotal removal of the tumor, total removal was possible in $29.8 \%$ of cases, the biopsy in $12.7 \%$. However, when the data mechanogram intraoperative monitoring, tracking boundaries resection should be considered intraoperatively shift occurs in the brain. Thus, during the operation the displacement of the center of the white substance formed resection cavity, and the distance from the area of operations to the point of passing the white matter pathways is reduced. Conclusions: Removal of intracranial tumors under the control of intraoperative monitoring using data from DT-MRI tractography, the conditions of intraoperative accuracy and safety of surgery to minimize surgical trauma, helps reduce the risk of neurological deficit and the quality of life of patients.

\section{9}

\section{Microsurgical management of pediatric craniopharyngioma}

Tatsuki Oyoshi, Shingo Fujio, Mika Habu, Manoj Bohara, F.M. Moinuddin, Ryosuke Hanaya, Hirofumi Hirano, Hiroshi Tokimura, Kazunori Arita

Department of Neurosurgery, Graduate School of Medical and Dental Sciences, Kagoshima University, Kagoshima, Japan

Introduction: The optimal treatment of pediatric craniopharyngioma has been debated for many years. We reviewed the treatment results and outcomes in children who underwent microsurgical treatment for pediatric craniopharyngioma in our institute over the last decade.
Methods: Eight children among 47 patients with craniopharyngioma treated and followed at Kagoshima University Hospital between 2002 and 2012 were reviewed. We also assessed pathological findings of the removed specimens for both growth hormone receptor (GHR) and insulinlike growth factor-1 receptor (IGF-1R) using immunohistochemistry. Results: Eight children underwent microsurgical near-total removal. The mean age at surgery was 6.3 (range 2-10) years. Transcranial approach was performed in 6 patients, transsphenoidal approach in one, and both approaches in one patient. Gamma knife surgery (GKS) was performed in 4 patients less than 6 months after first surgery, 2 patients at recurrence more than one year after first surgery. Regional recurrences occurred in 3 cases, and additional GKS was performed in two patients, second microsurgical removal in one patient. Panhypopituitarism occurred in two patients, diabetes insipidus in two, after surgery. Severe obesity with a transient electrolyte imbalance occurred in one case. Two patients with GH deficiency underwent GH replacement therapy (GHRT). GHR and IGF-1R were expressed in tumor tissue. All patients had normal mental development.

Conclusion: Almost all children with craniopharyngioma after near-total removal had good outcomes, due to GKS and various hormone replacement therapies. However, the continuous radiological examination is needed for those patients, especially the patients with GHRT.

120

Cerebellar pontine angle oligodendroglioma: The first reported case in a child

Christopher Parks, Sandra Perez, Conor Mallucci

Department of Paediatric Neurosurgery, Alder Hey Hospital, Liverpool, United Kingdom

Introduction: Oligodendrogliomas have a reported incidence in children of $1-6 \%$. The vast majority arise in the cerebral hemispheres. We report the first paediatric case of a grade II oligodendroglioma arising in the cerebellar pontine angle (CPA).

Case: This 15-year-old boy presented with one month history of worsening torticollis, headaches, nausea and double vision. Examination demonstrated ataxia, nystagmus and a paresis of left trochlear nerve. Brain MRI showed a predominantly solid, left sided CPA tumour with some cystic and calcified areas and irregular enhancement. There was a degree of brainstem compression but MR of the spine was normal. A near total excision of the tumour was achieved during surgery limited by a difficult plane of cleavage around the cranial nerves and brain stem. Histology reported an oligodendroglioma and cytogenetically confirmed a $1 \mathrm{p} 19 \mathrm{q}$ deletion. Post-operatively the patient made a good recovery in terms of his presenting symptoms and did not exhibit any new neurological deficits. MRI performed 5 months after resection demonstrated growth of the residual CPA tumour requiring further surgery. Complete gross resection was achieved utilising intra-operative MRI.

Discussion: A few cases of CPA oligodendroglioma have been reported in adults but never previously in children. Prognosis of supratentorial oligodendroglioma in children is good with complete excision and is more positive with $1 \mathrm{p} 19 \mathrm{q}$ deletion but the natural history of CPA disease is unknown. Perhaps the early progression of residual indicates aggressive disease in this case.

\section{1}

Microsurgical resection of giant thalamic/hypothalamic pilocytic astrocytoma in children: a single center experience

Aurelia Peraud, Lena Armbruster, Mathias Kunz, Jörg-Christian Tonn Department of Neurosurgery, Ludwig-Maximilians-University Munich, Munich, Germany

Introduction: Standard treatment for pilocytic astrocytomas in surgically accessible areas of the brain is gross total resection being the strongest prognostic factor for progression free and overall survival. In contrast, 
microsurgical resection of thalamic/hypothalamic pilocytic astrocytoma is still a topic of controversial discussion due to the risk of significant procedure related morbidity. We present our experience with microsurgical resection of large thalamic/hypothalamic tumors.

Methods: 4 children were operated upon in 2011 and 2012, one girl and three boys with a mean age of 5.5 years (range 9 months to 11 years).

Results: Clinically, 3 children presented with progressive hemiparesis and one with headaches, fatigue, seizures and ocular bobbing. In two children, the tumor had been pretreated with chemotherapy with subsequent progression - they required shunt implantation prior to surgical resection. Tumors derived mainly from the thalamus in three and from the hypothalamus in one patient. Resection was accomplished via a subfrontal $(n=2)$, a transtemporal $(n=1)$ and an interhemispheric approach. Near gross total resection could be achieved in all cases. Postoperatively, all children had aggravated transient neurological deficits including hemiparesis, double vision, hypothalamic insufficiency. However, symptoms recovered within 12 months under intensive rehabilitation to reach nearly the preoperative status. Only the hypothalamic tumor of the youngest child showed progression within 4 months and he received chemotherapy according to the HIT protocol. Conclusions: Despite the risk of significant transient morbidity, microsurgical tumor resection of large thalamic and hypothalamic tumors is feasible with good clinical longterm recovery and tumor control.

\section{2}

Long-term survivor of stage 4 neuroblastoma with central nervous system recurrence

Jun Sakuma ${ }^{1}$, Yuka Matsumoto ${ }^{1}$, Kiyoshi Saito ${ }^{1}$, Shinichiro Ohara ${ }^{2}$, Hideki Sano ${ }^{2}$, Atsushi Kikuta ${ }^{2,3}$

${ }^{1}$ Department of Neurosurgery, Fukushima Medical University; ${ }^{2}$ Department of Pediatrics, Fukushima Medical University; ${ }^{3}$ Division of Pediatric Oncology, Fukushima Medical University Hospital Cancer Center

Neuroblastoma (NB) is the most common extracranial solid tumor of childhood. Central nervous system (CNS) metastasis occurs in $1-16 \%$ in patients with $\mathrm{NB}$, and it has been reported that its prognosis is poor, especially with stage $4 \mathrm{NB}$ with MYCN amplification. We report a 2 years 6 month old girl with a cerebellar metastasis of NB who had been diagnosed into stage 4 disease with MYCN amplification at 8 months old. Conventional chemotherapy, radiation, high dose chemotherapy with autologous peripheral blood stem cell transplantation and 13-cis-retinotic acid administration were performed as initial therapy. Eight months after the first complete remission, a solid tumor was found in her right cerebellar hemisphere in follow-up CT. Because she was asymptomatic, we selected stereotactic radiosurgery (SRS) with a peripheral dose of 20Gy under informed consent to her parents. However, the size of tumor was expanded by bleeding in a tumor, we resected this lesion and achieved second remission. The tumor was intraparenchymal lesion without any attachment of the adjacent dura. Postoperative course was uneventful, however, 21 months after the cerebellar relapse, mastoid antrum metastasis was detected. Due to the isolated bone relapse and fair general condition, aggressive chemotherapy and local radiation were performed. Following those therapies, she maintains a third complete remission for 93 months. The CNS metastasis of the NB with MYCN amplification is relatively rare and its prognosis is expected extremely poor. However, if the CNS relapsed lesion is localized, the survival time could be prolonged by the aggressive surgical resection.

\section{3}

Long-term outcome after surgery for brainstem tumors in children Francesco Sala, Daniele Nuzzi, Miriam Mulino, Barbara Masotto, Albino Bricolo, Carlo Mazza

Institute of Neurosurgery, Pediatric Neurosurgery, University Hospital, Verona, Italy

Introduction: Except for diffuse gliomas, focal brainstem tumors are amenable to surgical treatment. Yet the long-term follow-up of surgical series is rarely reported. We reviewed long-term results in 51 children (mean age 10 years, range 1-17) operated on for brainstem tumors between 1994 and 2007. Materials and methods: Reviews of clinical charts and outpatient clinic reports as well as phone interviews were used to collect clinical, neuroradiological, and quality of life data. Based on the pre-operative MRI, tumors were classified as focal $(n=20)$, exophytic $(n=23)$, diffuse $(n=6)$ or cervicomedullary $(n=2)$. Tumor removal was classified as total $(n=16)$, subtotal $(n=32)$ or partial/biopsy $(n=3)$. Pilocytic astrocytomas were the most common histological diagnosis $(n=29)$. Radiotherapy $(\mathrm{RT})$ and/or chemotherapy (CHT) were used in 16 patients.

Results: On February 2013, 7 patients are lost to follow-up, 13 are dead (all malignancies except 2), while 31 are alive at a mean follow-up of 137 months (range 64-219). Of these 31 patients, 8 have no evidence of disease, 21 have stable disease, 2 have small progression, and only two received adjuvant therapies following surgery. Eight patients are cognitively impaired and overall neurological impairment is absent/mild in 12 , moderate in 7 , while 5 are fully dependent on external support for daily activities. Currently, 9 patients are attending school, 12 are employed, 10 unemployed.

Conclusion: While the prognosis of diffuse and/or malignant brainstem tumors remains dismal, surgery alone warrants long-term survival in the majority of low grade tumors, especially pilocytic astrocytomas. Among survivors who became adults, about half are employed.

124

The nature of double concomitant myxopapillary ependymoma.

Report of a case

J. Francisco Salomão ${ }^{1}$, Cecília V. de Andrade $^{2}$, Antonio R. Bellas ${ }^{1}$, Tatiana Protzenko ${ }^{1}$

${ }^{1}$ Division of Pediatric Neurosurgery, ${ }^{2}$ Department of Pathology, Fernandes Figueira National Institute of Health for Children, Woman and Adolescent, Rio de Janeiro, Brazil

Mixopapillary ependymomas are almost exclusively seen at the conus medularis / filum terminale /cauda equine region and usually present as solitary space-occupying lesions. The authors report the case of a 14-yearold boy with double concomitant myxopapillary ependymoma, proximal and caudal on the filum terminale in which a totally gross removal was achieved in two stages. This form of presentation is rare and so far we know just two similar cases have been previously reported in children. The true nature of these lesions is controversial and while some argue they are independ lesions developing synchronously, other consider it related to metastatic seeding. A critical review on multiple spinal myxopapillary ependimomas is made.

\section{5}

Pineal region tumours: A single institution experience over 30 years Mano Shanmuganathan, Greg James, Kim P. Phipps, Dominic N. P. Thompson, Richard D. Hayward, N. Owase Jeelani, William F. J. Harkness, Kristian Aquilina

Department of Neurosurgery, Great Ormond Street Hospital for Children, London, United Kingdom

Introduction: Pineal region tumours are uncommon and account for less than $1 \%$ of all primary brain tumours in children.

Methods: Casenote/database review of all patients with pineal region tumours under oncological and neurosurgical service from 1978-2012. Demographics, treatment, histology, endocrine status, recurrence, educational status and survival were recorded.

Results: 89 patients were identified. Male to female ratio was 2:1 (4.6:1 for germ cell tumours). Median age at diagnosis was 8.4 years (range 0 15.8years). Commonest pathology was germ cell tumours (GCT) $(n=45)$ $>$ pineal parenchymal tumour $(n=21)>$ glioma $(n=10)$, atypical teratoid/ rhabdoid tumour (ATRT) $(\mathrm{n}=2) .14$ tumours were multifocal. $76 \%(68 /$ 89) were high-grade. $79 \%$ (70/89) underwent biopsy or resection. $80 \%$ $(61 / 89)$ received radiotherapy. No endoscopic procedures were 
undertaken prior to $1994 ; 73 \%$ of patients with hydrocephalus $(22 / 30)$ underwent permanent ventriculo-peritoneal (VP) shunting. After 1994, $50 \%(25 / 51)$ underwent endoscopic third venticulostomy (ETV); 12 of these had positive endoscopic biopsies. Pre-1994, only 1 patient with GCT had positive tumour markers; no surgery was carried out. Post-1994, $12(12 / 32)$ patients with GCT had positive tumour markers and no resection. Long term endocrine replacement was required in 19 patients. Survival was best for GCT and worst for ATRT.

Conclusion: This series from a single institution reflects evolution of paediatric pineal tumour management over the last 30 years. Endoscopy, tumour markers and progress in adjuvant therapy have led to substantial changes to the management pathway.

\section{6}

Optic pathway gliomas - from childhood to adulthood

Ben Shofty ${ }^{1,2}$, Felix Bokstein ${ }^{3}, Z_{\text {Zvi Ram }}{ }^{1}$, Liat Ben-Sira ${ }^{4}$, Sigal Freedman ${ }^{1}$, Anat Kesler ${ }^{5}$, Shlomi Constantini ${ }^{1,2}$

${ }^{1}$ Division of Neurosurgery, ${ }^{2}$ The Gilbert Israeli Neurofibromatosis Center, ${ }^{3}$ Neuro-Oncology Service, ${ }^{4}$ Pediatric Radiology Unit, ${ }^{5}$ NeuroOphthalmology Unit, The Tel-Aviv Medical Center, and the Sackler Faculty of Medicine, Tel-Aviv University

Background: Optic pathway gliomas (OPG) are considered relatively benign pediatric tumors. Adult OPG can be divided into two groups; adult patients with tumors diagnosed in childhood, and adult patients diagnosed during adulthood. In this study we have characterized the clinical course of patients belonging to these two groups.

Methods: We retrospectively collected clinical and imaging data for all adult OPG patients who have been monitored in our medical-center between 1990 and 2012.

Results: Twenty-two adult patients were included. Age distribution at diagnosis varied widely $(6 \mathrm{~m}-66 \mathrm{y})$, as did age at last follow-up (18y$74 \mathrm{y})$. Ten patients were diagnosed at adulthood, 12 in childhood. Of the patients that were diagnosed at childhood, 6 had radiological progression during childhood, and 3 of those suffered visual impairment. From this group of 6 patients, 1 had further radiological progression during adulthood accompanied by additional visual decline, 2 patients had additional visual decline during adulthood despite no signs of radiological progression. Of the 6 patients whose tumors were stable during childhood, all 6 remained stable during adulthood. Out of 10 patients diagnosed at adulthood, 6 patients suffered visual deterioration; in 5 of them a concomitant radiological progression was noted. Two patients were diagnosed with high-grade gliomas; both died of their disease.

Conclusion: OPGs may be active during childhood or adulthood. Those patients that experienced anatomical activity during childhood are prone to continue experiencing active disease during adulthood. A significant percentage of patients diagnosed with low-grade OPG at adulthood may suffer progression, or visual decline, or both.

\section{7}

A rare case of a symptomatic bilateral choroid plexus xantogranuloma in a boy treated with MRI guided laser ablation Zulma Tovar-Spinoza ${ }^{1}$, David Carter ${ }^{1}$, Klaus Werner ${ }^{2}$

Departments of ${ }^{1}$ Neurosurgery and ${ }^{2}$ Neurology. SUNY Upstate Medical University, Syracuse, NY

Intracranial xanthogranulomas are rare benign tumors that occur most commonly as choroid plexus xanthogranulomas, which rarely become symptomatic. Exact etiology is still a unclear. 17 cases of intracranial have been reported in the literature, only one pediatric. We present the case of a 4 y old boy with a history of intractable severe headaches on recumbent position that would wake him up screaming from sleep. Complete headaches evaluation including measuring intracranial pressure, MRI, MRA, MRV, neuro-opthalmologic was performed. The patient did not respond to medications. Headaches will only alleviate with the head in 45 degrees positioning. MRI showed a mirror-looking choroid plexus xantogranulomas on the bilateral occipital horns. The lesions were biopsied and treated minimally invasive with MRI guided laser ablation. The patient became headache free. To our knowledge this is the second case of a symptomatic bilateral choroid plexus xantogranuloma reported in a child and the first one treated with thermal laser ablation.

\section{8}

Irradiation-induced meningiomas following therapy of brain tumors in childhood

Wouter Verfaillie, J. Menten, B. Depreitere, Johannes van Loon, Steven De Vleeschouwer, J. Goffin, Frank Van Calenbergh

Department of Neurosurgery and Radiotherapy, Universitary Hospitals Leuven, Belgium

Introduction: Cranial radiotherapy may cause several late complications. Secondary tumors are a late and serious complication.

Material and methods: Since 1995, we prospectively identified all patients presenting at our department with possible irradiation-induced meningiomas who received radiotherapy before the age of 17 .

Results: 19 patients with 23 meningiomas were identified. Age varied between 2 and 16 years when receiving radiotherapy. The interval between radiotherapy and diagnosis of the secondary meningioma was 12 to 34 years (average 23). The primary tumors were low-grade gliomas (14), ependymoma (1) and medulloblastomas (4). There were multiple meningiomas in 2 patients. Meningiomas presented with epileptic seizures (4), neurologic deficit (3), vomitus (2). 16 Meningiomas were asymptomatic and discovered on a surveillance scan. The meningiomas were all WHO grade I except one WHO grade II. Other post-irradiation complications were documented: skin lesions, white matter disturbances and cavernous angiomas. One patient died due to tumor progression in the fossa posterior despite 2 surgical interventions and chemotherapy. A second patient died of a new brain lesion, 11 years after meningioma resection, due to an irradiation-induced glioma. No serious complications in relation to the meningioma occurred in the other patients.

Conclusion: An irradiation-induced meningioma seems to be a frequent complication after radiotherapy, although the exact risk cannot be calculated from this series. Radiotherapy turns out to be effective in treating the primary tumor in this population. Patients treated with brain irradiation in childhood are candidates for lifelong and regular surveillance scanning.

\section{9}

Individualized treatment of childhood large craniopharyngioma in peroperative period: Cases report and literature review Yang Zhao, Jie Ma

Department of Pediatric Neurosurgery, Xinhua Hospital, Shanghai JiaoTong University School of Medicine, Shanghai, China

Introduction: Craniopharyngiomas are rare tumors. They in the pediatric population exhibit particularly insidious characteristics, namely the frequent large size, the possible association with hydrocephalus and a high recurrence rate. More consideration should be applies to the postoperative management, which may be weighted by serious complications. Total tumor removal is associated to a higher rate of short-term and longterm complications. We discuss the individualized treatment of childhood large craniopharyngioma in peroperative period on the basis of our experiences.

Material and Method: Twenty three patients with large craniopharyngioma were admitted and had operations between 2008 and 2011. We review and analyze the gender, age, computer tomography (CT) / magnetic resonance imaging features, surgical approaches and complications in the peroperative period.

Results: Sixteen boys and seven girls are included and mean age is 5.07 years (aged 7 months to 14 years). All cases combined with hydrocephalus. 
Fourteen patients had calcification lesions in CT scanning. All these patients were treatmented by using microsurgeries with total resection in 10 cases, subtotal resection in 5 cases and part resection in 1 case. The surgical approaches contained pterion approach (6 cases), anterior transcallosal approach (4 cases) and combine approach (6 cases). All patients had complications with different degrees, such as diabetes insipidus, defits of the visual function, severe obesity and damage of the Willis circle. Conclusions: In the recent times, "minimally invasive" surgical management of craniopharyngiomas coupled with various types of adjuvant therapies appears to offer a valid surgical option in the management of this tumor. Individualized treatment in peroperative period is more and more important.

\section{0}

Clinical review and prognosis analysis of the central nervous system embryonal tumors in children

Yang Zhao, Jie Ma

Department of Pediatric Neurosurgery, Xinhua Hospital, Shanghai JiaoTong University School of Medicine, Shanghai, China

Introduction: Discuss the clinical characteristics and treatment methods of the central nervous system embryonal tumors in children. Compare the prognosis and its influence factors between atypical teratoid / rhabdoid tumor (AT/RT), medulloblastoma $(\mathrm{MB})$ and primitive neuroectodermal tumors (PNET).

Material and method: A retrospective study was conducted on history, pathology results and follow-ups of patients with CNS embryonal tumors at our center from 2006.1 to 2012.1 .

Results: In this study, 49 cases were collected, with 11 cases of AT/RT, 24 cases of MB and 14 cases of PNET. Overall survival (OS) is $20.4 \% \pm$ $10.0 \%$ with mean survival time (MST) of 22.1 months \pm 4.2 months. OS of patients receiving postoperative chemo/radiotherapy is $39.8 \% \pm 18.5 \%$ with MST of 37.0 months \pm 6.7 months. OS of those receiving both postoperative chemo/radiotherapy and tumor gross total/subtotal resection reaches $76.2 \% \pm 10.5 \%$ with MST of 49.5 months \pm 5.9 months. Outcome of AT/RT is the worst, while MB the best in this study with MST of 3.49 months \pm 0.66 months and 33.84 months \pm 6.51 months respectively. OS of patients younger than 3 years old is $23.7 \% \pm 9.4 \%$ with MST of 12.86 months \pm 3.02 months, while OS of patients older than 3 years old is $40.3 \% \pm 13.2 \%$ with MST of 31.89 months \pm 6.51 months.

Conclusions: CNS embryonal tumors are highly malignant with poor outcomes. Postoperative chemo/radiotherapy can significantly prolong survival in these patients, and tumor GTR/SR will further improve patients' prognosis. AT/RT is the most malignant CNS embryonal tumor with worse outcome than MB and PNET. Therefore, total/subtotal resection combined with postoperative chemotherapy and/or radiotherapy can effectively improve the prognosis of the patients with CNS embryonal tumors in children.

\section{Others}

\section{1}

Electrostatic discharges affect the validity of registered values in intracranial pressure monitors

Morten Andresen ${ }^{1}$, Marianne Juhler ${ }^{1}$, Ole C. Thomsen ${ }^{2}$

${ }^{1}$ Clinic of Neurosurgery, Copenhagen University Hospital, Copenhagen, Denmark; ${ }^{2}$ Department of Electrical Engineering, Technical University of Denmark, Lyngby, Denmark

Background: Intracranial pressure (ICP) monitoring is used extensively in clinical practice, and as such, the accuracy of registered ICP values is paramount. Clinical observations of unphysiological changes in ICP, have called the accuracy of registered ICP values into question. Subsequently, we have tried to determine, if the ICP monitors from major manufacturers were affected by electrostatic discharges (ESD), if the changes were permanent or transient in nature, and if the changes were modified by the addition of different electrical appliances normally used in the ICU environment.

Methods: We established a test setup in the neurointensive care unit using a large container filled with isotonic saline, creating a phantom patient. ICP monitors were sequentially lowered into the container, and subjected to a predefined test battery of ESDs.

Results: We evaluated five pressure monitors from four manufacturers. Three monitors containing electrical circuitry at the tip of the transducer were all affected by electrostatic discharges. We saw clinically significant permanent changes in the reported ICP values for one pressure monitor, and temporary deflections for two other monitors. The monitors had different levels of sensitivity to discharges at low voltages.

Conclusions: Our results explain some of the sudden shifts in ICP seen in the clinical setting. We did not, however, find a clear deflection pattern related to the addition of electrical appliances. We recommend instituting policies for reducing the risk of subjecting patients to electrostatic discharges in the neurointensive care setting.

\section{2}

Accidental misplacement of a central venous catheter located in intracranial subarachnoid space: a case report Cristina B. Bleil ${ }^{3}$, Jorge W. J. Bizzi ${ }^{1}$, André Bedin ${ }^{2}$, Fabiano P. Soares ${ }^{3}$, Maira C. Velho ${ }^{3}$, Rodrigo Roncaglio ${ }^{3}$, Marcelo C. Vione ${ }^{3}$

${ }^{1}$ Chief of Pediatric Neurosurgery Department, Hospital da Crianca Santo Antonio, Porto Alegre, Brazil; ${ }^{2}$ Neurosurgeon at Pediatric Neurosurgery Department, Hospital da Crianca Santo Antonio, Porto Alegre, Brazil; ${ }^{3}$ Resident at Pediatric Neurosurgery Department, Hospital da Crianca Santo Antonio, Porto Alegre, Brazil

Introduction: Right internal jugular vein catheterism is used due to its accessibility and low frequency of complications. To our knowledge, misplacement of a central venous catheter in intracranial subarachnoid space has not been reported before. We present a rare case of an accidental misplacement of a central venous catheter intracranial subarachnoid space. Case report: One-month-old girl presented tachypnea in the first hours after birth. The child was diagnosed with neonatal pneumonia and transferred to ICU for mechanical ventilation. A central venous catheter was placed in the right jugular vein for parenteral nutrition and drug infusion. Five days later, the patient presented status epilepticus, persisting despite the treatment. The child was then transferred to an ICU at our hospital for evaluation. The cerebrospinal fluid exam demonstrated a high glucose level $(>1000 \mathrm{mg} / \mathrm{dL})$. The patient underwent a CT scan, which showed pneumocephalus, bilateral frontoparietal subdural collections and the proximal portion of the central venous catheter located in the intracranial subarachnoid space. The catheter was withdrawn without complications. The seizures resolved, controlled with phenobarbital. The child presents neurological sequelae, with persistent clonus in lower limbs, generalized spasticity, nistagmus and absence of visual contact with the examiner.

Conclusion: Central venous catheter misplacement in the subarachnoid space is a very rare complication. The infusion of medication and parenteral nutrition in the subarachnoid space may provoke severe neurological deficits and post-traumatic seizures. As far as we know this is the first case of accidental catheterization of subarachnoid space described in literature.

\section{3}

Accidental misplacement of a central venous catheter located in lateral medullary-cerebellar cistern: a case report Cristina B. Bleil ${ }^{3}$, Jorge W.'J. Bizzi ${ }^{1}$, André Bedin ${ }^{2}$, Maira Cristina Velho $^{3}$, Fabiano P. Soares ${ }^{3}$, Rodrigo Roncaglio ${ }^{3}$, Marcelo C. Vione ${ }^{3}$ ${ }^{1}$ Chief of Pediatric Neurosurgery Department, Santo Antonio Childrens Hospital, ${ }^{2}$ Neurosurgeon at Pediatric Neurosurgery Department, Santo Antonio Childrens Hospital, ${ }^{3}$ Resident at Pediatric Neurosurgery Department of Santo Antonio Childrens Hospital, Porto Alegre, Brazil 
Introduction: Right internal jugular veincatheterism is used due to its accessibility and low frequency of complications. To our knowledge, misplacement of a central venous catheter in intracranial subarachnoid space has not been reported before. We present a rare case of an accidental misplacement of a central venous catheter in lateral medullary-cerebellar cistern.

Case report: One-month-old girl presented tachypnea in the first hours after birth. The child was diagnosed with neonatal pneumonia and transferred to ICU for mechanical ventilation. A central venous catheter was placed in the right jugular vein for parenteral nutrition and drug infusion. Five days later, the patient presented status epilepticus, persisting despite the treatment. The child was then transferred to an ICU at our hospital for evaluation. The cerebrospinal fluid exam demonstrated a high glucose level $(>1000 \mathrm{mg} / \mathrm{dL})$. The patient underwent a CT scan, which showed pneumocephalus, bilateral frontoparietal subdural collections and the proximal portion of the central venous catheter located in the right lateral medullarycerebellar cistern. The catheter was withdrawn without complications. The seizures resolved, controlled with phenobarbital. The child presents neurological sequelae, with persistent clonus in lower limbs, generalized spasticity, nistagmus and absence of visual contact with the examiner.

Conclusion: Central venous catheter misplacement in the subarachnoid space is a very rare complication. The infusion of medication and parenteral nutrition in the subarachnoid space may provoke severe neurological deficits and post-traumatic seizures. This is the first case of accidental catheterization of subarachnoid space described in literature.

\section{4}

\section{Use of an ultrasonic bone curette (SONOPET) in pediatric} neurosurgery

Akira Gomi ${ }^{1}$, Hirofumi Oguma ${ }^{2}$, Fumihiro Arai $^{2}$, Rintaro Kuroda ${ }^{2}$, Yasushi Sugawara ${ }^{3}$

${ }^{1}$ Pediatric Neurosurgery, ${ }^{2}$ Neurosurgery, ${ }^{3}$ Plastic Surgery, Jichi Medical University

Introduction: Recently, ultrasonic bone curettes (UBCs) have been introduced for spinal surgery and skull base surgery. We describe the clinical application, procedure, and efficacy of UBC in pediatric neurosurgery.

Methods: From July 2007 to March 2013, we performed 72 pediatric neurosurgical procedures using UBC with a SONOPET UST-2001 as follows: laminectomy or laminoplasty for spinal tumors and lipomas (42 cases); foramen magnum decompression (FMD) (seven cases); and multidirectional cranial distraction osteogenesis (MCDO) for craniosynostosis (23 cases). We could select the tip from the following three types: scalpel-type tip; longitudinal vibration tip; and longitudinal-torsional vibration tip.

Results: The gap for bone curettage is very thin with the scalpel-type tip (0.7-mm wide), and thus useful for laminoplastic laminotomy in spinal surgery for infants because of good osteogenesis after laminoplasty. The longitudinal vibration tip is effective for fine bone curettage near the dura in FMD. The longitudinal-torsional vibration tip is more powerful, and therefore suitable for laminectomy or MCDO. Although we treated only two cases $(2.8 \%)$ of dural damage in the first and third cases of MCDO in our series, no subsequent iatrogenically induced injuries were encountered. Considering potential complications of mechanical injuries using ultrasonic technique, intermittent usage and cotton protection during use are recommended. Although previous reports have described UBC as time-consuming for removing bone in adults, UBC can easily cut the soft, thin bones of children in a short time. Therefore, combination with standard drilling is not needed.

Conclusions: This system appears to be versatile, safe, and efficient for pediatric neurosurgery.
135

Histological structure of the walls of cavernous sinus in normal human fetuses

Yusuf $\mathrm{Izci}^{1}$, Cahit Kural ${ }^{2}$, Gulcin Guler Simsek ${ }^{3}$, Erhan Arslan ${ }^{4}$

${ }^{1}$ Department of Neurosurgery, Gulhane Military Medical Academy, Ankara, Turkey; ${ }^{2}$ Department of Neurosurgery, Hakkari Military Hospital, Hakkari, Turkey; ${ }^{3}$ Department of Pathology, Kecioren Research and Education Hospital, Ankara, Turkey; ${ }^{4}$ Department of Neurosurgery, Kecioren Research and Education Hospital, Ankara, Turkey

Cavernous sinus (CS) is a complex anatomical structure which involved in many pathological processes. The walls of CS has been investigated by many authors in adults but medial (MW) and lateral (LW) walls are not examined in fetal cadavers. The aim of this study is to elucidate the development of these fine structures in human fetuses. The histological examination of MW and LW was performed in 15 normal human fetuses. Eleven fetuses were female and 4 were male. The gestational age was ranged between 14 and 35 weeks. The weight was ranged between $180 \mathrm{~g}$ and $1750 \mathrm{~g}$. The walls samples (2 MW and $2 \mathrm{LW}$ from each fetus) were obtained by microsurgical technique and underwent histological examination. Each wall was examined for collagen, elastic fiber, ganglion, peripheral nerve and vessel. A total of 60 wall samples ( $30 \mathrm{MW}$ and $30 \mathrm{LW}$ ) were examined in 15 fetuses. Collagen tissue was observed in all walls with different densities. Elastic fibers were observed only in 3 wall samples (2MW and $1 \mathrm{LW}$ ). Ganglion was detected in 11 samples ( 9 in LW and 2 in MW). Peripheral nerve was found in 28 walls (18 LW and $10 \mathrm{MW}$ ). Vessel was observed in 51 samples (26 LW and $25 \mathrm{MW}$ ). The structure of LW and MW of the CS in fetuses is mainly composed of collagen tissue while some elastic fibers are supported this tissue. Vessels are present during the intrauterine life, but peripheral nerves and ganglions are still under development.

\section{6}

\section{Image-guided neuroendoscopy in supratentorial intraventricular} cysts

Roberto Jaimovich, Federico Auad, Sebastián Jaimovich, Graciela Zúccaro Hospital Nacional de Pediatría J.P.Garrahan, Buenos Aires, Argentina

Surgical approaches to supratentorial intraventricular cysts (SIC) have changed from microsurgery to neuroendoscopy usually combined with frameless navigation. The objective of this study was to retrospectively evaluate the results of a neuroendoscopic approach in 25 patients with symptomatic cysts located within the lateral ventricles, anterior and posterior $3^{\text {rd }}$ ventricle, and septum pellucidum. Ependymal, choroid plexus, arachnoid, and pineal cysts were included. Tumoral, parasitic, post-infection, and post-haemorrhagic cysts were excluded. It was always possible to perform the surgery through a single entry point. The initial target was the lateral ventricle even when the size was small. A thinner endoscope was used, but with a size large enough to allow the use of more than one instrument at the same time. If navigation is used, head and equipment position may allow a permanent detection of the endoscope. The objective is to communicate the cyst with the ventricle. Resection of the membrane is not necessary. In septum cysts it is enough to open one wall only. Anterior $3^{\text {rd }}$ ventricle cysts were resolved with a wide opening of the rostral membrane. Small ventricles are a specific indication for image-guided neuroendoscopy. Neither a microsurgical approach nor shunt placement were necessary in these patients.

137

\section{Waterjet dissection in pediatric patients}

Doerthe Keiner ${ }^{1}$, Michael R. Gaab ${ }^{2}$, Juergen Piek ${ }^{3}$, Joachim Oertel ${ }^{1}$

${ }^{1}$ Department of Neurosurgery, University Medical center Saarland, Homburg/Saar, Germany; ${ }^{2}$ Department of Neurosurgery, Klinikum Region Hannover GmbH, Hannover, Germany; ${ }^{3}$ Department of Neurosurgery, University of Rostock 
Objecitve: Waterjet dissection enables precise brain tumour dissection and tumour separation from the surrounding intact brain parenchyma with preservation of even very small blood vessels. Of the authors group, more than 250 various neurosurgical pathologies were operated on with the waterjet dissector so far. In this collective, nine patients aged 3 to 18 with different intracranial pathologies have been treated.

Methods: Waterjet dissection was applied on 4 girls and 5 boys with gliomas, vascular or embryonal tumours, or temporal lobe epilepsy. Application (dissection and debulking) and its usefulness in each procedure was analyzed. Additionally, potential complications due this technique were evaluated.

Results: The technique was optimal for dissection and tumor debulking in vascular tumours and gliomas due to their soft or highly vascularized morphology. Dissection and debulking was possible with pressures of $6-12$ bar. In epilepsy surgery, pressures of $4-$ 10 bar enabled subpial dissection with preservation of the arachnoid membrane and small vessels. Application of the waterjet for debulking the embryonal tumor was not found to be advantageous due to its' firm morphology. No side effect like increased postoperative seizure or infection rate was observed.

Conclusion: The technique was well suited for application in pediatric patients. An advantage in tumour dissection and debulking was observed in gliomas and in epilepsy surgery. In the solid PNET, higher jet pressures were required for debulking, and the waterjet was applied only scarcely. The device was especially helpful in pathologies that require low pressures for dissection as well as debulking of tumours.

138

Clinical application of SPM in pediatric patients with Moyamoya disease using MRI ASL

Tomoko Matsuzaki ${ }^{1}$, Reizo Shirane ${ }^{1,3}$, Yoshihisa Shimanuki ${ }^{2}$, Yasuyuki $\mathrm{Taki}^{4}$, Hiroshi Hashizume ${ }^{4}$, Ryuta Kawashima ${ }^{4}$

${ }^{1}$ Department of Pediatric Neurosurgery, Tohoku University Graduate School of Medicine; ${ }^{2}$ Department of Radiology, Miyagi Children's Hospital; ${ }^{3}$ Department of Neurosurgery, Miyagi Children's Hospital; ${ }^{4}$ Division of Developmental Cognitive Neuroscience, Institute of Development, Aging and Cancer, Tohoku University

Arterial Spin Labeling (ASL) with 3T-MRI is becoming increase in clinical application. In the present study, we aimed to determine the usefulness and problems of ASL for CBF measurement in neurologically normal pediatric patients with Moyamoya disease.

Patients and Methods: rCBF values in Moyamoya patients $(n=20)$ after surgical treatments were measured quantitatively using ASL perfusion MRI, and analyzed their correlation. These data were also compared with rCBF database obtained from healthy children $(n=133)$ studied at Tohoku University. We resized the ASL perfusion image voxel size to match that of the MPRAGE images. Perfusion images were co-registered to corresponding gray-matter segments in native space and nonlinearly normalized by applying the corresponding normalization parameter. ROIs (Hemisphere, Temporal, Parietal, Occipital, Frontal, Thalamus) were set using WFU PickAtlas and the $\mathrm{rCBF}$ values were analyzed by SPM8 in MATLAB.

Results: The rCBF values in neurologically stable pediatric patients with moyamoya disease were significantly decreased in all ROIs compared with the values in normal children.

Conclusion: These results suggested that analyzing rCBF by SPM provides useful information for treatment strategy. Considering the correlation in cerebral blood flow and functional recovery from ischemic symptoms of developing brain, we may clarify the mechanism of neuronal plasticity commonly observed in pediatric Moyamoya patients.

\section{9}

Developing paediatric neurosurgery applications for mobile devices Ganesalingam Narenthiran ${ }^{1}$, Dorothy Lang ${ }^{2}$, Ash Singhal ${ }^{2}$

${ }^{1}$ Department of Neurosurgery, Wessex Neurological Centre, University Hospital Southampton, Southampton; ${ }^{2}$ Department of Neurosurgery, British Columbia Children's Hospital, Vancouver, Canada

Objective: Mobile devices including smart phone and tablet computers are increasingly used in all walks of life including medicine to improve efficiency. The objective of the project was to custom develop applications related to paediatric neurosurgery for Android and iOS devices without help from IT professionals. Our aim was to develop a calculator for computing the 'ETV Success Score'.

Method: We used Adobe Flash CS6 software to write the 'ETVSS calculator; using Action script 3.0. The software was developed using MacBook Pro (13"; early 2011) running OS 10.8.3. The Adobe Flash CS6 was then used to compile the source code to publish applications for Android devices, iOS devices, web-application, Windows computers and Apple computers.

Results: The application developed runs on Android devices, web, Windows computers and Apple computers. The application can also run on iOS devices (iphone, iPod touch) however, for distribution further license and approval is required from Apple company.

Conclusion: Development of custom paediatric neurosurgery application for mobile devices, as well as web and desktop computers is possible, without assistance from IT professionals; Adobe Flash CS6 provides a means for writing one program and compiling applications for multiple platforms including mobile devices.

140

Stereotactic biopsy in children - a multicenter retrospectic study Julian Rathert ${ }^{1}$, Rüdiger Gerlach ${ }^{1}$, Klaus Hamm ${ }^{1}$, Gerhard Marquardt ${ }^{2}$ ${ }^{1}$ Klinik für Neurochirurgie, HELIOS Klinikum Erfurt, Germany; ${ }^{2}$ Klinik und Poliklinik für Neurochirurgie, JWG-Universität Frankfurt, Germany

Objective: Stereotactic biopsy is a safe and effective procedure. Spare data are published so far concerning stereotactic biopsy in children. We report on children undergoing stereotactic biopsy in two neurosurgical centres over the last 30 years.

Method: A retrospective study was conducted on stereotactic procedures performed in children between 1982 and 2010 in two major neurosurgical centres. Medical records were analyzed concerning stereotactic systems used, results of biopsy and complications.

Results: 31 stereotactic procedures were performed in both centres in 28 children aged 1 to 16 years. In 26 children biopsy of a mass lesion were the primary purpose, in the others biopsy of different lesions or diagnostic puncture of cysts. In two children biopsy of gliomas were performed twice, to rule out, respectively confirm a secondary malignant transformation. In one child puncture of a hypothalamic cyst for decompression was performed twice after initial diagnostic puncture. Stereotactic biopsy revealed sufficient diagnosis in all cases, except for one child with unspecific diffuse signal changes (MRI). Decompression of cysts was successful in all procedures. Postoperative imaging was performed in 20 procedures, showing in four cases haemorrhages, without clinical relevance. In one patient younger than two years, postoperative MRI revealed a small impression fracture, due to the stereotactic frame. Postoperative complication occurred in one patient as wound infection.

Conclusion: Stereotactic biopsy is a safe and effective diagnostic procedure in children. In infants, stereotactic procedures are possible, but care has to been taken for a sufficient stability of the cranium to avoid calvarian fractures. 
141

The use of intraoperative neurophysiological monitoring in foramen magnum decompression: a preliminary experience

Mano Shanmuganathan ${ }^{1}$, Greg James ${ }^{1}$, Arjel Lejarde ${ }^{2}$, Nil Haliasos ${ }^{1}$, Dominic N. P. Thompson ${ }^{1}$

${ }^{1}$ Department of Neurosurgery, Great Ormond Street Hospital, London;

${ }^{2}$ Department of Neurophysiology, Great Ormond Street Hospital, London

Aims: To evaluate the use of intraoperative neurophysiological monitoring (IONM) during surgery for Chiari 1 malformation specifically: 1) Do IONM changes correlate with clinical and radiological outcomes. 2) Could IONM be used as an indication for need for dural opening.

Methods: Retrospective case study of all Chiari 1 malformations that underwent foramen magnum decompression (FMD) over 24 months. Electrophysiological parameters: Somatosensory evoked potentials (SSEP) and brainstem auditory evoked potentials (BAEP) were monitored. Three surgical time points (baseline, bony and dural opening) were correlated to changes in SSEP and BAEP. Outcomes measures: Changes in latencies in BAEP $(>0.3 \mathrm{~ms}$ considered significant $)$ and latencies of N20 SSEP ( $>1.0 \mathrm{~ms}$ considered significant). Radiological and clinical outcome at 6 month follow-up.

Results: Twenty seven children underwent FMD. Mean age was ten years (range 7 months to 16 years). Eighteen patients had pre-operative syrinx. Nineteen had both bony and dural opening and eight underwent bony decompression alone. Twenty patients showed improvement in evoked potential following surgery. Thirteen showed improvement following bony decompression. Baseline to closure increases in latencies of wave $\mathrm{V}$ were $0.24 \mathrm{~ms}$ and interpeak latency I-V in BAEP were $0.21 \mathrm{~ms}$. Latencies of N20 SSEP were significant at $1.26 \mathrm{~ms}$. Post-operative clinical improvement was noted in twenty five patients. Sixteen patients had improvement in their syrinx. Headache resolution was noted in twenty three patients. No post-operative complications were noted.

Conclusion: IONM changes correlate with clinical and radiological outcomes. IONM may be used as an indication for need for dural opening however further research is required.

\section{2}

Rare occurrence of a spontaneous subarachnoid hemorrhage in an infant: A case report of basilar tip aneurysmal rupture and review of the literature

Venita Simpson ${ }^{1}$, Amar Swarnkar ${ }^{2}$, Zulma Tovar-Spinoza ${ }^{1}$

Departments of ${ }^{1}$ Neurosurgery and ${ }^{2}$ Neuroradiology, SUNY Upstate Medical University, Syracuse, NY, USA

Introduction: Intracranial aneurysms are rare in childhood accounting for $0.5 \%-4.6 \%$ in patients $0-18$ years of age, and even more infrequent in infants accounting for $0.1 \%$. Intracranial aneurysms in children differ from those in adults in relation to sex predilection, location, morphology, size, multiplicity, etiology, rate of spontaneous aneurysm thrombosis, susceptibility to post hemorrhagic vasospasm with our without delayed ischemic neurologic deficits, and management. We present a rare case of an infant with subarachnoid hemorrhage secondary to basilar tip aneurysm rupture and a review and the most recent literature.

Case report: A previous healthy full term 7-month-old female presented to the emergency department with sudden neurological deterioration and seizures. There was no family history of vascular disease, trauma, or infection. CT head revealed diffuse subarachnoid hemorrhage along the sylvian fissures and basal cisterns with intraventricular extension and hydrocephalus. MRI/MRA revealed extensive bilateral cortical infarct and swelling within the parieto-occipital region and a $2 \mathrm{~mm}$ basilar tip aneurysm. The patient had a right frontal EVD placed emergently and underwent diagnostic cerebral angiogram and a two-step coiling of her basilar tip aneurysm. Management consisted of normotensive, normovolemic status and intracranial pressure monitoring.
Conclusion: Aneurysms in infants are rare; therefore specific treatment protocols for this population do not exist. Most aneurysms in patients $<1$ year old are located in the MCA (42\%) and in the posterior circulation $(24 \%)$. The cause of these aneurysms is unclear and further studies and reports are needed.

\section{3}

Pediatric empyema: A persistent problem in the setting of an improved outcome

Alan Siu, Maria Pena, Tiffani DeFreitas, John Myseros, Suresh Magge, Chima Oluigbo, Robert Keating

Departments of Neurosurgery and Otolaryngology Children's National Medical Center George Washington University School of Medicine Washington, DC, USA

Introduction: Morbidity and mortality of empyemas in children have been historically high (10-20\%) despite aggressive treatment. Recently these rates have declined with the adoption of more aggressive surgical/medical management. Despite advances in early detection, we continue to observe difficulties in identifying children at risk, but have noted a continued improvement in outcome. We subsequently sought to characterize current-day risk factors associated with morbidity and mortality as well as outcomes for intracranial empyemas in children.

Methods: A retrospective review (1990-2013) of children presenting with intracranial empyemas was undertaken. Demographic/perioperative data for neurosurgery and ENT were collected and analyzed for statistical significance/relevance.

Results: 41 children (mean age 10yrs), predominantly male, presented with subdural (76\%) and epidural (24\%) empyemas. Headache was the most common complaint, with seizures, Potts puffy tumor, obtundation and fevers also noted. Associated sinusitis and periorbital cellulitis were often implicated. Triple antibiotics were administered immediately upon presentation and neurosurgical evacuation was performed within 48 hours on $93 \%$ of patients, with $17 \%$ receiving more than one surgery. Concomitant endoscopic sinusotomies were performed on $35 \%$. The most common causative organism was streptococcus intermedius(39\%) with multiple organisms frequently present. Sinus thrombosis was seen in $12 \%$. At two year follow-up, there was one mortality(unrelated) and minimal morbidity.

Discussion: A multi-disciplinary approach utilizing early and aggressive neurosurgical/ENT intervention has dramatically improved overall mortality and morbidity for pediatric empyema. Repeat surgery and addition of sinusotomies may also contribute to these improvements.

\section{4}

Adolescents with mild health problems and their mothers

Vaitsa Giannouli ${ }^{1}$, Nikolaos Syrmos ${ }^{2}$

${ }^{1}$ School of Psychology, Aristotle University of Thessaloniki, Thessaloniki, Greece; ${ }^{2}$ School of Medicine, Aristotle University of Thessaloniki, Thessaloniki, Greece

Background: In the present paper we examine the possible relationship between the scores for anxiety and depression of mothers and the perception of illness for their sick teenage children. Also we examine the possible relationship of scores for anxiety and depression of mothers and children and the quality of relationship that they have with their doctor.

Materials and Methods: We administered the State-Trait Anxiety Inventory (STAI), the Center for Epidemiological Studies-Depression Scale (CES-D), the Parental Stress Scale, the Brief Illness Perception Questionnaire and the Questionnaire for the Relationship between Doctor and Patient to 50 mothers (without an official diagnosis of mental health problem, aged 35-60). The same questionnaires (with the exception of the Parental Stress Scale) were administered to their teenage sick children (age 13-18, 20 boys and 30 girls). 
Results: We found statistically significant negative correlations between the scores of STAI/CES-D/Parental Stress Scale and the Brief Illness Perception Questionnaire/ Questionnaire for the relationship between doctor and patient, for both mothers and children.

Conclusions: Our data suggest that the parents' (and specifically mothers') perception of their child's disease can be largely linked to mood disorders that they personally face or that even a brief illness of a child (such as a seasonal influenza) can seriously influence the mood of the caregivers. Also the quality of relationship between doctor and patient (both for parents and adolescents) is negatively associated with the scores on the previous scales.

\section{5}

Our four year experiences with electromagnetic neuronavigation during pediatric neurosurgery procedures

Petr Vacek, D. Štepánek, V. Pribán

Neurosurgery Department, Charles University Hospital, Plzen, Czech Republic

Introduction: The most frequent pediatric neurosurgical procedures are shunt and endoscope surgeries in children suffered from hydrocephalus and arachnoidal cysts. These procedures still have a relatively high percent of technical complications. Planning of approach for neuroendoscopy and intracranial catheter inserting require a high accuracy. Therefore we generally use neuronavigation in neurosurgery but during it rigid fixation of head is necessary. For that reason electromagnetic (EM) neuronavigation was developed.

Methods: From January 2009 we used EM neuronavigation during 40 procedures in infants with age range from 3 days to 21 months. We performed 19 V-P shunt surgeries, 7 etVs, 4 endoscopic cyst fenestrations, 5 combined procedures - endoscopic fenestration + shunt surgery in one session, 3 intracranial abscess evacuations and 2 intracranial tumor resections.

Results: All procedures were technically successful. We have no problem with accuracy during the navigation. We solved 6 shunt related complications - 5 obstructions and one infection. Outcomes of the other procedures were uneventful.

Conclusions: EM neuronavigation enables us to perform neurosurgical procedures in infants with the same accuracy of intraoperative navigation as in adults.

\section{6}

Diagnostic value of sequential ventriculo-cisternography for the treatment of arachnoid cysts

Sonja Vulcu ${ }^{1}$, Sebastian Senger ${ }^{1}$, Heiko Körner ${ }^{2}$, Joachim Oertel ${ }^{1}$

${ }^{1}$ Neurochirurgische Klinik, Universitätsklinikum des Saarlandes, Homburg; ${ }^{2}$ Institut für Neuroradiologie, Universitätsklinikum des Saarlandes, Homburg

Introduction: Arachnoid cysts treatment is based on clinical and radiological findings. Nevertheless, outcome of surgery differs significantly. In the present work, the value of ventriculo-cisternography in surgical decision making is evaluated.

Methods: All ventriculo-cisternographies performed in patients with arachnoid cysts in Homburg between September 2011 and April 2013 were retrospectively analysed. Ventriculography was either performed with repetitive MR- or CT-imaging.

Results: Twenty-six ventriculo-cisternographies were performed in ten patients. Seven patients received CT- and/or MR-ventriculographies preoperatively. In all patients there was no or only minimal cyst filling in the scans after 30, respectively 90, 180 and 360 minutes. All patients underwent surgical treatment. Clinical symptoms resolved and the postoperative ventriculography revealed sufficient contrast agent enhancement. One patient underwent cyst fenestration without preoperative ventriculography. Symptoms postoperative improved and MR- ventriculography showed cyst communication with filling of the cyst after 180 minutes. In one patient with IFV the MR-ventriculography showed insufficient filling of the ventricle. The patient underwent aqueductoplasty and the symptoms resolved. In the last patient, the ventriculography revealed sufficient cyst communication, therefore surgical treatment was abandoned. Two patients presented with renewed complaints. MR- and CT-ventriculography showed reclosure of the cyst connection and recurrent cyst fenestration was performed.

Conclusion: Ventriculo-cisternography presents a valuable tool in the treatment of arachnoid cysts. Consideration of the radiological findings in the different time slots may influence the surgeon's action and the postoperative clinical outcome. Even though CT-ventriculography generates more detailed and observer-independ images, the higher radiation exposure must be concerned - particularly in pediatric patients.

147

Differential expression of adhesion, migration and inflammation genes in mouse and human neural progenitor cells- functional impact on their in vitro phenotype

Nancy Wang ${ }^{2}$, Catherine Brégère ${ }^{1 *}$, Raphael Guzman ${ }^{1,2}$

${ }^{1}$ Neurochirurgische Klinik \& Brain Ischemia and Regeneration Group Universitätsspital Basel; ${ }^{2}$ Department of Neurosurgery-Stanford University School of Medicine, CA, U.S.A

*Presenter

Introduction: Transplantation of neural progenitor cells (NPCs) is a feasible treatment for neurological disorders. The sources of NPCs currently used in preclinical research are heterogeneous. Our goal was to compare them in terms of migration, adhesion and inflammatory properties, aspects that relate to their therapeutic effectiveness.

Methods: Gene and protein expression analysis of migratory and immunerelated factors was performed in human embryonic stem cells derived neural progenitor cells (hESC NPCs), mouse immortalized NPCs (ms C17.2), and mouse fetal derived NPCs (ms F NPCs). The functional significance of these results was tested via in vitro adhesion and migration assays.

Results: Transcriptome analysis revealed that (i) extracellular matrix and adhesion genes were largely and highly expressed in all mouse NPCs, and $25 \%$ were line specific; $40 \%$ of these genes were expressed in both mouse and human NPCs, and few were species-specific. (ii) In contrast, mouse and human NPCs expressed a limited subset of genes encoding chemokines and their receptors. Among those expressed, 32\% were shared in mouse NPCs, and 29\% were common between species; a fewer percentage was either line or species-specific. The secretome of inflammatory molecules was highly variable in NPCs. Functional differences between NPCs were revealed in their adhesion to E-selectin and migration response to SDF-1 or MCP-1.

Conclusions: Overall, this study is the first to document extensively the repertoire of migratory, adhesive and inflammatory mediators in NPCs lines from different origins. Expression of these factors correlates fairly with their adhesion and migration behavior.

\section{8}

Enlarged parietal foramina

Yasuo Yamanouchi ${ }^{1}$, Hideyuki Ohshige ${ }^{2}$, Kyoichi Iwata ${ }^{2}$, Kunikazu Yoshimura $^{2}$, Akoi Asai ${ }^{2}$

${ }^{1}$ Tsurumiryokuchi Hospital; ${ }^{2}$ Kansai Medical University

Enlarged parietal foramina (EPF) ( $>5 \mathrm{~mm})$ are variable ossification defect in the both parietal bones. We experienced two cases with no clinical symptoms and present radiological findings.

Case1: 5 -year-old boy was born with neonatal asphyxia and bony defects in the both parietal area were noticed at birth. Delayed closure of anterior fontanel was noticed. There was no sign and symptoms without low height at the first medical examination. CT scan showed delineate bony defect in the both parietal bone. Sagittal MRI demonstrated falcine vein. 
Case2: 10-year-old girl visited outpatient clinic with headache and symptoms of common cold. CT scan showed bony defect in the both parietal bone. Sagittal MRI demonstrated falcine vein. Headache was evaluated no correlation with enlarged parietal foramina. Both cases have been followed in the outpatient clinic.

Discussion: Enlarged parietal foramina are symmetric bony defect and believed to be a developmental anomaly of parietal bone ossification. Prevalence of 1:15000 to 1:50000 has been reported. EPF are hereditary condition and genes associated with this condition have been identified (ALX4,MSX2). Most cases are asymptomatic and headache is chief complaint in some cases. EPS has been reported to show cortical infolding, falcine vein and hypoplasia or atresia of the straight sinus. Cranioplasty is recommended for this condition at risk for injury in selected patient.

\section{Spine}

\section{9}

Flip osteoplastic laminotomy flap for excision of long segment spinal tumours in children

Azmi Alias, Mohammed Saffari Mohammed Haspani

Department of Neurosurgery, Hospital Kuala Lumpur, Malaysia

Introduction: Long segment laminectomy for excision of spinal tumours in children is known to be associated with significant rate of spinal deformity and instability. We propose our surgical technique of using a flip osteoplastic laminotomy flap to provide adequate access and achieved complete excision of long segment spinal tumours in children.

Methods: Patient is positioned prone on bolster. Intraoperative image intensifier is used to localize the upper and lower level of spinal tumour. Posterior midline incision is made preserving the posterior interspinous and supraspinous ligaments, exposing both sides of lamina. Highspeed drills is used to make a small opening on superior border of the highest lamina and inferior border of the lowest lamina under microscopic visualization, ensuring intact dura. Laminotomy is performed on both sides using a low profile high-speed drills system, incorporating the ligamentum flavum. The interspinous ligament on cephalic end is cut with scissor and osteoplastic flap with intact interspinous ligament at the caudal end is raised, flip caudally and hold in position with towel clip. Following removal of spinal tumours, the osteoplastic laminotomy flap is fixed back to it's corresponding lamina and the spinous process at the cephalic end with absorbable suture.

Results: All patients had complete or near complete removal of spinal tumour with good postoperative recovery. There were no complications related to this approach.

Conclusion: Flip osteoplastic laminotomy flap provide optimum access to long segment spinal tumours in children emphasizing on preservation of normal architecture of posterior elements in pediatric spine.

\section{0}

\section{Cervicomedullary decompression (CMD) of foramen magnum} without duraplasty in achondroplastic children - a low risk procedure that may improve patients' functions but with a fairly high recurrence rate

Bengt Gustavsson, Chen Wang, Ulrika Sandvik, Erik Edström

The Institution for Clinical Neuroscience, The Karolinska University Hospital, Stockholm, Sweden

Achondroplasia is a common inherited dwarfism characterized by short stature, macrocephaly, and various skeletal abnormalities. Stenosis at the level of the cervicomedullary junction commonly occurs. Symptoms associated with the stenosis, such as hypotonicity, pain, incontinence, apnoea and sudden death, are well known. For surgical treatment there is no consensus concerning the patient selection and surgical approach. We present our experiences using CMD without duraplasty. 27 children with achondroplasia underwent $32 \mathrm{CMD}$, that is about $60 \%$ of all children with achondroplasia in our reception area. Mean age at surgery was 3 years (1 week - 12 years). The indications for surgery were "prophylactic" in 23 children and progressive ataxia, progressive quadriparesis, central apnea and sudden respiratory arrest respectively in four children. MRI was done preoperatively in all children. The sagittal diameter varied between $2 \mathrm{~mm}$ and $9 \mathrm{~mm}$. Surgery included a suboccipital craniectomy, atlas laminectomy and cutting of eventual fibrous bands on the dura. One child had a shunt placement at the same surgery as the CMD-procedure. Another five children received a shunt in a separate operation. Even though symptoms improved, in all symptomatic and a few of the asymptomatic children after surgery, the foraminal stenosis recurred in 5 children. (follow-up 3 months - 15 years). The intraoperative findings at reoperation was similar to those found at the primary surgery and no duraplasty was done except in one child. Even if there is a significant risk of recurrence, we will still recommend CMD if there is a foraminal stenosis on MRI.

\section{1}

Spinal lipoma and VACTERL association

Masahiro Kameda ${ }^{1,2}$, Nobuhito Morota ${ }^{1}$, Hideki Ogiwara ${ }^{1}$, Masashi Kitagawa $^{1}$, Natsu Sasaki ${ }^{1}$

${ }^{1}$ Division of Neurosurgery, National Center for Child Health and Development, ${ }^{2}$ Department of Neurological Surgery, Okayama University Graduate School of Medicine

Introduction: VACTERL is an acronym for "vertebral defects, anal atresia, cardiac anomaly, tracheoesophageal fisturas, radial and renal dysplasia, and limb defects." Since spinal lipoma is sometimes found in the course of further investigations for VACTERL association, we reviewed our operative notes for spinal lipomas.

Methods: From 2002 to 2013, 481 patients with spinal lipoma had 505 operations in our department. Among 481 patients with spinal lipoma, anal atresia was present in 31 patients, and 12 of these patients were VACTERL association. We analyzed the type of spinal lipoma and neurological findings and outcome in these patients.

Results: The age of these patients were ranged from four month to 13 years old. Regarding the type of spinal lipoma, three patients had caudal type of lipoma, eight patients had filar type, and one patient had thickend filum. All patients presented tethering, three patients presented syringomyelia, eight patients presented neurogenic bladder, and three patients presented lower limb motor disorder. Untethering was performed in a preventive manner. Bulbocavernous reflex (BCR) was performed as an intraoperative monitoring and the response of BCR was preserved throughout the operation. Postoperative courses were uneventful. Conclusion: Untethering operation for spinal lipoma is worth considering for the patients with VACTERL association.

\section{2}

Clinical outcomes and considerations of the lumbar interbody fusion technique for lumbar disk disease in adolescents

Dae-Woong Kwon, Sung-Uk Kuh

Department of Spinal Neurosurgery, Gangnam Severance Hospital, Yonsei University of Medicine, Seoul, Republic of Korea

Introduction: The posterior lumbar interbody fusion (PLIF) and transforaminal lumbar interbody fusion (TLIF) techniques are commonly used surgical methods for wide indications such as degeneration or trauma. Although they are rarely required for lumbar disk disease in younger patients, there are a few children and adolescents who are indicated for PLIF or TLIF for other reasons, such as congenital severe stenosis with or without lumbar instability that requires wide decompression or severe bony spur that need to be removed. In such cases, different 
pathophysiology and outcomes are expected compared with adult patients.

Methods: We retrospectively reviewed data of 23 patients who underwent PLIF or TLIF surgery when less than 20-years-old. Clinical and radiographic outcomes were assessed during a mean of 36.4-month follow-up period. The indications of lumbar interbody fusion, success of fusion, complications, and visual analog scale (VAS) were analyzed.

Results: Radiographs of all patients taken 6 months after the surgery showed fusion. Clinical outcome was also satisfactory, with improvement of VAS score from 7.7 preoperatively to 2.3 at 6 months after surgery. Only one patient had reoperation due to adjacent segment disease.

Conclusions: For adolescent patients with severe bony spur, massive central disk rupture, or severe spondylolisthesis, lumbar interbody fusion surgery has good surgical outcome with few complications.

\section{3}

\section{Intramedullary tumors in patients with neurofibromatosis type 2}

Anna C. Lawson McLean, Steffen K. Rosahl

Department of Neurosurgery, HELIOS Klinikum Erfurt, Germany

Introduction: Data on natural growth of tumors in patients with neurofibromatosis type II (NF2) are rare, but indispensible for a management that focuses on the preservation of neurological function. The objective of this study was to evaluate radiographic data on intramedullary tumors in this patient population.

Methods: From a group of 49 NF2 patients those with intramedullary tumors $(\mathrm{n}=26 ; 53.06 \%)$ were selected. Tumor volumes were determined by volumetric extrapolation after segmentation in data sets (iPlanNet software, Brainlab, Munich). The minimum follow-up time was set at 6 month and digital T2 weighted MRI scans had to be available from at least two different investigations. Relevant pharmacological interventions (Bevacizumab, Octreotid, Goserelin) were taken into account.

Results: 23 patients, 8 males and 15 females, were included in this analysis (mean follow-up $39.3 \pm 27.4$ months, range 11 - 98 months). The mean age at initial evaluation was $29.6 \pm 8.4$ years (range $19-51$ years). Patients harbored 94 intramedullary neoplasms, 49 of which were assessable for growth rate analysis. Overall, 29 tumors (59.2\%) exhibited growth, 11 tumors $(22.4 \%)$ did not show any growth, 9 tumors $(18.4 \%)$ decreased in size. 3 patients required microsurgical resection of their intramedullary tumors.

Conclusions: NF2-associated intramedullary tumors usually grow slowly. However, as there is little room for compensation and little functional regeneration in the spinal medulla, timing is crucial in the initiation of treatment in order to preserve important functional capacities and there is no alternative to a follow-up from childhood to death for these patients.

\section{4}

Augmentation laminoplasty in children: How effective is it? Naomi V. Slator ${ }^{1}$, Pasquale Gallo ${ }^{1}$, Ravi Vemaraju ${ }^{1}$, Guirish A. Solanki ${ }^{1,2}$ ${ }^{1}$ Department of Paediatric Neurosurgery, Birmingham Children's Hospital, Birmingham, UK; ${ }^{2}$ University of Birmingham, Birmingham, UK

Objectives: There is paucity of information on indications and outcomes of laminoplasty in children. We evaluate our experience with laminoplasty in paediatric practice. Design. Retrospective review of case notes and radiology.

Subjects: 71 (36 boys and 35 girls) consecutively operated children by a single surgeon, aged between $4 \mathrm{~m}-15$ years.

Methods: Laminoplasty was performed using a foot-plate protected cutter appropriate for bone thickness. In younger children laminoplasty was secured bilaterally using 2-0 PDS and augmented fixation using 1-0 PDS from last spinous process accross to the caudal spinous process or to the distal sacral anchor. In older children 1.7- $2 \mathrm{~mm}$ titanium plates and 4 -
$5 \mathrm{~mm}$ screws were used holding the facet and the lamina together in an augmented canal state.

Results: Average follow-up was 61 (1-182) months. 80\% (57/71) underwent laminoplasty for treatment of occult neural tube defects and split cord malformations. $11 \%(8 / 71)$ for spinal tumours. $4 \%(3 / 71)$ for neuroenteric/ arachnoid cysts. $3 \%(2 / 71)$ Achondroplasia and 1.4\% (1/71) case of central disc prolapse. $4 \%(3 / 71)$ were in the cervical spine, $6 \%(4 / 71)$ were thoracic and $90 \%(64 / 71)$ in the lumbar spine. $85 \%(60 / 71)$ patients were secured using PDS alone. 15\% (11/71) cases were fixed using plates. No deformity or instability was identified in either the PDS or metal plate fixation groups. There were no known instances of construct failure.

Conclusions: Laminoplasty is an effective procedure without the significant risks for spinal deformity associated with laminectomy. In this series no child developed spinal deformity, fusion failure or required subsequent metal fixation.

\section{Trauma}

\section{5}

Craniocerebral gunshot wounds in pediatric population

Javier González Ramos, S. Vitar, R. González, Graciela Zúccaro

Neurosurgery Department, Hospital Nacional de Pediatría "Juan P. Garrahan", Buenos Aires, Argentina

Objective: There has been an increase in civilian gun violence since the last decade, with a disproportionately high increase occurring the pediatric population. The objective of this study is to describe and analyze a series of pediatric patients who underwent surgery for craniocerebral gunshot wounds.

Material and method: The study is a retrospective review of the clinical charts of patients with cranial gunshot injuries at Hospital de Pediatria "Prof Dr Juan P Garrahan". Neurological and radiological findings were studied as well as the outcome of the patients.

Results: Fifty one patients were admitted and 48 were operated on always with general anesthesia. Mean age of the children was 7 years. Thirty five patients were male and sixteen female. Thirteen children sustained transhemipheric injuries, thirteen extradural injuries, 11 tangential injuries, 6 bihemipheric injuries and 4 transventricular injuries, 2 posterior fossa injuries. Child's age or gender, the mode of injury and the presence of extracerebral injuries were not found to be significantly associated with deaths. Mortality rate was $21 \%$ with a mean follow-up of 48 months. Conclusion: The pediatric population tends to demonstrate more favorable outcomes following intracranial gunshot injury when compared with the adult population. We conclude that almost all cranial gunshot patients should initially receive aggressive surgical therapy with a previous CT scan.

\section{6}

Frontobasal lesions in preterms: An underestimated complication of nasotracheal intubation?

Amin Hashemi ${ }^{1}$, Johannes Pöschl ${ }^{2}$, Andreas Unterberg ${ }^{1}$, Heidi Bächli ${ }^{1}$ ${ }^{1}$ Clinic of Neurosurgery, University Hospital, Heidelberg; ${ }^{2}$ Department of Neonatology, University Hospital, Heidelberg

Objective: Nasotracheal intubation (NTI) has been a well established method for maintaining an artificial airway in preterms. Concomitant complications are rarely reported in the literature. The presented complications with frontobasal injuries in preterms are not yet described and maybe misdiagnosed and therefore underestimated.

Methods: We describe two cases of female preterms (26 and 24 week of pregnancy) with a tube perforation through the cribriform plate and frontobasal skull following NTI. Repeated intubations after birth were needed due to breathing problems. In one case three months after discharge an initially not diagnosed rhino liquorrhea and excessive snorring 
led to further investigations and the diagnosis of a big frontonasal encephalocele. In the other case intracerebral hemorrhage with CSF circulation disorder occurred.

Results: All cases were operated immediately after diagnosis (218 and 87 days after birth). Surgical treatment includes covering of the anterior skull base with an anteriorly pedicled periosteal flap. In one case an additional shunting was performed due to a resulted trapped ventricle after two months.

Conclusions: Despite the often very urgent nature of airway problems in preterms the placement of a nasotracheal tube should be approached in careful manner. The incompleted ossification of the frontobasal bone might be an essential risk factor. A failure of a tube-positioning with frontobasal injury should be always considered where a spontaneously liquid leaking from the nose occurs - suspicious for rhino liquorrhea - or multiple intubations were done. Early recognition and appropriate intervention is the only way to avert a fatal outcome.

\section{7}

Bi-coronal separated skull fracture: a unique fatal type of traumatic head injury in infancy

Sung Kyoo Hwang, Seong Hyun Park

Department of Neurosurgery, Kyungpook National University Hospital, Daegu, Korea

An infantile skull is maliable and sutures are tightly adherent to the underlying dura and venous sinus. These characteristics in association with the small amount of total blood volume result in a specific fatal type of the skull fracture which is unique in infancy. The authors report a fatal type of infant head injury in which we should pay attention to the possibility of massive bleeding during operation.

Case report: A 23-month-old female baby presented with semicomatose mentality after suffering fall-down injury from the second floor. The plain skull films showed bi-frontal skull fracture crossing the midline. The computed tomography scan revealed an acute epidural hematoma in the right fronto-temporo-parietal area with severe brain edema causing significant midline brain shift. In the emergency operation, scalp incision exposed massive bleeding from the fracture site. The bleeding was identified arising from the lacerated and widely separated sagittal sinus below the fracture line. The patient underwent hypovolemic shock immediately after the scalp incision. The patient died two days after the trauma and surgery. Senior author experienced one more such a fatal case in which the patient deteriorated immediately after scalp incision (not reported due to loss of films).

Conclusion: We report a case of unique fatal type of skull fracture in infancy and discussed the pathogenesis and significance of the fracture. Special concern should be paid during operation of this type of fracture.

\section{8}

Cerebral concussion and brain injuries in the paediatric population Ch. Iliadis, Nikolaos Syrmos, D. Arvanitakis

Neurosurgery Department, Venizeleio General Hospital, Heraklion, Crete, Greece

Objective: To conduct a topic review of studies related to cerebral concussion and brain injuries, as an aid to improving decision-making and outcomes. We describe existing knowledge about the epidemiology and etiology of concussions in this population.

Methods: We review the literature to provide a perspective on the incidence and definition of and the management guidelines for mild to severe brain injury in paediatric population. Metabolic changes resulting from cerebral concussion and the second-impact syndrome are reviewed. The most commonly used definition of concussion are those proposed by Cantu, American Academy of Neurology, Virginia Neurologic Institute. Results: Earlier definitions required a loss of consciousness (LOC) associated with a head injury for diagnosis of a concussion. Multiple studies have questioned the significance of LOC as a prerequisite, citing LOC rates as low as $10 \%$. In the Emergency Department (ED), the use of neuroimaging studies to evaluate concussion is a source of much discussion. Because concussion is more a metabolic and functional disturbance than a structural injury to the brain both CT, MRI, and fMRI are useful tools for to determine intracranial pathology in the children.

Conclusion: Neuropsychological testing seems to be an effective way to obtain useful data on the short-term and long-term effects of brain injury. The clinicians who cares for patients less than 16 years old with concussion must consider that return not only to everyday activities but also to academic rigors of school, with each environment having its own specific concerns that must be taken into account.

\section{9}

"Sk8 is great?" An unusual case of chronic subdural hematoma and review of the literature on sport-related minor head injuries in youths

Jacek Szczygielski, Sina-Maria Gund, Ralf Ketter, Karsten Schwerdtfeger, Joachim Oertel

Department of Neurosurgery, Saarland University, Medical Center, Homburg/Saar, Germany

Introduction: The increasing number of youths takes regular part in recreational activities requiring exposition on high acceleration / deceleration or jump / landing forces. The risk of sustaining a head injury during these sports is unknown and probably still underestimated.

Case report: We describe the case of a 17-year-old male patient who sustained a large chronic and subacute subdural hematoma without a loss of consciousness. No single episode of head injury was reported, however the patient admitted to be a passionate skateboarder. An extensive radiological and laboratory evaluation ruled out vascular malformation or coagulopathy as the underlying cause. Because of the size of the hematoma and contralateral symptoms, surgical evacuation was required.

Discussion: The repetitive head injury has been typically attributed to contact sports including boxing, ice-hockey or American football. Critically evaluating our case and reviewing the literature, minor repeated head traumas due to acceleration/decelaration probably yielding to leakage of bridging veins must be considered as the cause of the described hematoma. Conclusions: Although only few may consider skateboarding / inline skating a contact sport, previous data suggest that it is a common cause of sports-related injuries. In addition, as our case report demonstrates, there is a risk of intracranial hematoma formation even without a fall or direct blow to the head. This issue is of special importance for young patients with known congenital intracranial pathology (such as vascular malformation or arachnoidal cyst) seeking neurosurgical advice on participating in sport activities.

\section{0}

Analysis of skull fracture in mild pediatric head trauma

Takumi Yamanaka ${ }^{1}$, Atsuko Harada ${ }^{1}$, Hidetsuna Utsunomiya ${ }^{2}$, Mami Yamasaki $^{1}$

${ }^{1}$ Department of Pediatric Neurosurgery, Takatsuki General Hospital, Osaka, Japan; ${ }^{2}$ International University of Health and Welfare, Fukuoka, Japan

Introduction: Skull fracture is the most common form of head trauma in children, and a risk factor of intracranial injuries. The purpose of this study is to elucidate the criteria for applying imaging diagnosis in mild pediatric head trauma.

Methods: 446 patients were enrolled between April 2012 and March 2013. The mean age was 3 years and 9 months. Clinical features of all the patients and fracture patients were evaluated. 3DCT was performed in 259 patients $(58 \%)$, and $\mathrm{CT}$ findings were analyzed using AquriusNet software (TeraRecon, Inc.). 
Results: Skull fractures were observed in 20 cases of all the patients (4.5\%). Mechanisms of fracture were falling (16 cases), hitting ( 1 cases), pushing ( 1 cases), and abuse ( 2 cases). Short distance falls less than 3 feet developed fractures in 4 cases. More intracranial injuries were found in the patients with a fracture $(30 \%)$ than in those without $(2.9 \%)$. 3DCT revealed linear skull fracture in 17 cases $(85 \%)$ and depressed fracture in 3 cases $(15 \%)$. Of those performed 3DCT, accessory cranial sutures mimicking fractures were observed in occipital bone $(26.3 \%)$ and parietal bone $(5.8 \%)$.

Conclusions: Mild head trauma such as short distance fall can cause skull fracture. 3DCT clearly demonstrates fracture lines and quite useful for diagnosis, while we should be aware of the existence of accessory sutures mimicking skull fractures.

\section{Vascular}

\section{1}

Cavernoma of the third ventricle: study of a pediatric case and literature review

Jeremy Brodard $^{1}$, Benoit Jenny ${ }^{1}$, A. Etemad-Sajadi ${ }^{2}$, Olivier Vernet $^{3}$, Karl Schaller ${ }^{1}$, Bénédict Rilliet ${ }^{1}$

${ }^{1}$ Department of Neurosurgery, University of Geneva Medical Center, Switzerland; ${ }^{2}$ Department of Neurosurgery, Clinique La Source, Lausanne, Switzerland; ${ }^{3}$ Department of Neurosurgery, Clinique Cecil, Lausanne, Switzerland

Introduction: Cavernomas are vascular lesions which rarely occur in the ventricular system and especially in the pediatric population. We present the pediatric case of a cavernous malformation of the left lateral wall of the third ventricle and review the literature for pediatric population.

Methods: A 17-months-old child presented with symptoms of raised intracranial hypertension. Magnetic resonance imaging showed characteristic images of a cavernoma in the third ventricle associated with hydrocephalus. The patient underwent an uneventful surgical excision by a transcallosal and transventricular approach. Because of a recurrence from a little remnant after one year, a second surgery was needed with an excellent final radiological and clinical outcome. We then performed a literature review of 128 children with intracerebral cavernomas.

Results: We found in the literature 22 children with intraventricular cavernoma and only 7 in the third ventricle. The mean age was 8 years with two peaks at $0-2$ years $(26.8 \%)$ and $13-16$ years $(35.7 \%)$. There were no preponderance of gender with only $4.8 \%$ of cases with multiple lesions and 3 cases of familial form. The most prominent presenting symptom was intracranial hypertension $(57 \%)$, followed by seizures $(14,3 \%)$, visual disturbance $(14,3 \%)$ and endocrine disturbances $(14,3 \%)$.

Conclusions: The diagnosis of cavernoma in the pediatric population should be considered with lesions of the third ventricle. These lesions of benign nature have a high tendency for regrow, with recurrent hemorrhages, if incompletely removed. A total excision is mandatory, despite the difficult location.

\section{2}

\section{Spontaneous intracerebral hematoma in a 4-year-old boy}

Ralf Buhl, Metin Kalkan

Department of Neurosurgery, Städtisches Klinikum Solingen, Germany

Intracerebral hematoma in the pediatric patient is quite rare and often associated with tumor or vascular malformation. We present an interesting and rare case of a 4 year old boy with headache and a seizure. MRI of his head showed a $4 \mathrm{~cm}$ lesion in the right parieto-occipital region with edema. Preoperative differential diagnosis included tumor or hematoma associated with a vascular malformation. Intraoperatively the brownish encapsulated lesion could be resected completely. Histology showed an intracerebral hematoma. No tumor, no AVM, no cavernoma. The postoperative course was uneventful and the patient could be discharged home six days after surgery.

\section{3}

Subarachnoid hemorrhage in association with aortic coarctation a case report

Nazife Dinc ${ }^{1}$, Stephan Felber ${ }^{2}$, Jan S. Brunkwall ${ }^{3}$, Hans-Hermann Görge $^{1}$, Beate Schoch ${ }^{1}$, Dirk Smolders ${ }^{1}$

${ }^{1}$ Clinical Department of Neurosurgery, SKM Koblenz, Germany; ${ }^{2}$ Clinical Department of Radiology and Neuroradiology, SKM Koblenz, Germany; ${ }^{3}$ Clinical Department of Vascular Surgery, Medical University Cologne, Germany

Objective: Primary aortic coarctation is in 0,9 to $1,9 \%$ associated with cerebral aneurysma. This combination demands a multidisciplinary approach concerning management of SAH and order of treatments of the vessel diseases.

Methods: This case report presents a 27-year-old man with aortic coarctation and multiple aneurysms who suffered from SAH and reviews the literature available for this combination of vascular diseases.

Results: This previously asymptomatic 27 -year-old patient was presented with acute subarachnoidal hemorrhage (Hunt and Hess II, WFNS II, Fisher 3). Angiography showed multiple cerebral Aneurysms. Angiography also revealed a previously unknown coarctation of the aorta. We first treated the presumely ruptured aneurysma of the anterior communicating artery and two aneurysms of the left middle cerebral artery by a microsurgical approach and clipping. During the procedure blood pressure was kept in mild hypotension to maintain sufficient end-organ perfusion. Another aneurysm of the right middle cerebral artery was occluded via transfemoral endovascular approach using detachable coils. The patient developed vasospasms after day six and required scrutinous blood pressure regulation during intensive care management. After neurological rehabilitation he underwent treatment of the aortic coarctation.

Conclusion: This case report shall draw attention to the association of aortic coarctation with multiple cerebral aneurysms in young patients and will illustrate the importance of a multidisciplinary approach, especially concerning blood pressure management during aneurysm treatment and during the course of cerebral vasospasm.

\section{4}

A rare association of chronic subdural haematoma and autosomal polycystic kidney disease in children

Yassine El Hassani, Renato Almeida Gondar, Karl Schaller, Benoit Jenny Department of Neurosurgery, Geneva University Hospitals and Faculty of Medicine, Switzerland

Introduction: The association of autosomal dominant polycystic kidney disease (ADPKD) with cerebrovascular aneuryms and also with intracranial arachnoid cysts is well known. However, there are only a few cases reported of chronic subdural hematomas $(\mathrm{cSDH})$ with unknown origin described within the table of ADPKD. None of these cases is reported in children. To our knowledge this is the first case reported in a young adolescent with discrete clinical signs.

Clinical description: A sixteen-year-old patient, diagnosed with ADPKD and with a familiar history for aneurysmal rupture, was diagnosed with a left sided unilateral $7 \mathrm{~mm} \mathrm{cSDH}$ without any arachnoid cyst during a screening MRI. The adolescent presented no symptoms and the coagulation parameters were proved to be normal. There was no history of traumatic event in the past two months. The absence of symptoms as well as the size of the hematoma has allowed a conservative follow-up. The one-month MRI showed a significant enlargement of the cSDH. A cerebral angiography disclosed any vascular lesions. Urinary laboratory showed a micorhematuria. Two burr holes were performed with a successful recovery and disapperance of headaches after surgery. 
Conclusion: The association of a cSDH can be suspected in patients with ADPKD and unspecific neurological signs. The physiopathology remains though unclear; one explanation could be a distal capillary disease or a vasculitis.

165

Lateral situated pseudosinus pericranii (Pseudo-Stromeyer) in a child with surgical successful removal and embolization preoperative: case report and review of the literature Abraham Ibarra-de la Torre ${ }^{1}$, Gonzalo Solís-y-Maldonado ${ }^{2}$, Renato Vega-López ${ }^{3}$, Jorge Luis Balderrama-Bañares ${ }^{3}$

${ }^{1}$ Department of Neurosurgery of Hospital Central Sur de Alta Especialidad, PEMEX; ${ }^{2}$ Department of Neurosurgery of Hospital Angeles del Pedregal, ${ }^{3}$ Endovascular Neurologic Teraphy of Instituto Nacional de Neurologia y Neurocirugia Manuel Velasco Suarez, Mexico

Introduction: The sinus pericranii (SP) is a rare vascular anomaly of the vault, descriptions by Hecker in 1845 and Stromeyer in 1850 . Volkmann in 1950 classified the SP: true and pseudo, latter to be an angioma or cavernoma

Case: Child male, 1 year old age, without history of trauma; he born with vascular lesion left retro auricular that increased in volume for the time and for cries. In the neurological examination without relevance; with a mass under scalp left retro auricular area and measures $3.0 \times 3.5 \mathrm{~cm}$ that does not disappear totally with external pressure. Neuroimaging. Gray scale sonography, hypoechoic mass superficial to the skull; CT, lesion isodense in the scalp over left petrous bone, enhanced with contrast medium administration to the same degree as the sigmoid sinus and erosion of the external table; MRI, hiperintensity and posterior to intravenous contrast was given, the lesion enhanced. Cerebral angiography and embolization preoperative, vascular lesion with supply from posterior auricular artery. Surgery. Under general anesthesia, position supine, head contralateral inclination, incision retro auricular in form "C", with vascular lesion, solid, color purple, and supply for posterior auricular artery in the superior pole that was ligated with two silver hemoclip and total resection. Postoperative. The patient with good state (follow-up 51 months) and improvement without retro auricular mass. Pathology, sinuses lined by a layer of endothelial cells.

Conclusion: The treatment, when is required, is surgical; and the endovascular therapy few applied. We presented a case with a lateral pseudosinus pericranii y successful surgical resection and embolization preoperative.

166

Sunset phenomenon in a case of a combined hydrocephalus that masked intracranial varices of veins in an infant

Elke Januschek ${ }^{1}$, Judith Jochim ${ }^{2}$, Eleftherios Archavlis ${ }^{1}$, Pavel Timofeev $^{1}$, Peter T. Ulrich ${ }^{1}$

${ }^{1}$ Department of Neurosurgery, ${ }^{2}$ Department of Pediatric and Adolescent Medicine, Klinikum Offenbach GmbH, Offenbach, Germany

Introduction: Normally the mass effect of varices of intracranial veins can lead to hydrocephalus. We present a case where the venous anomaly was concealed by a complex hydrocephalus.

Case presentation: At the age of nine month $\mathrm{N}$. developed an increase in head circumference of $6 \mathrm{~cm}$, a tense fontanelle and a sunset phenomenon within 4 weeks. Ultrasound demonstrated enlarged external cerebrospinal fluid (CSF) spaces and a relevant midline shift. After completing the diagnostic evaluation by magnetic resonance imaging (MRI) we implanted a subduroperitoneal shunt with gravitational unit. Shortly after the surgical intervention the sunset phenomenon disappeared. In the control MRI we were surprised to see massively enlarged draining veins.
Conclusion: Not only varices can lead to hydrocephalus, but also hydrocephalus can conceal a preexisting venous anomaly. In this rare case a follow-up and exclusion of other vascular malformations is essential.

\section{7}

A mechanism of reversible tonsilar herniation associated with vein of Galen aneurysmal malformation after endovascular treatment

Kurihara Jun, Nishimoto Hiroshi, Sasano Mari

Department of Neurosurgery Saitama Children's Medical Center, Saitama, Japan

Introduction: A vein of Galen aneurysmal malformation with tonsilar herniation is rare. The mechanism of tonsilar herniation associated with congenital vascular malformations is unknown. We discussed about the mechanism of reversible tonsilar herniation associated with vein of Galen aneurysmal malformation.

Case presentation: A 10-month-old boy referred to our hospital for clinical examinations about his enlargeing head circumference. He had no significant developmental disorders and neurological examinations. Magnetic resonance imaging showed a occupying flow void lesion in pineal region and tonsilar herniation. Magnetic resonance angiography showed a dilated vein of Galen and straight sinus and digital subtraction angiography revealed some feeding arteries from bilateral lateral posterior choroidal arteries and a single draining vein though straight sinus. His cardiac functions and renal functions were normal range. He was diagnosed a vein of Galen aneurysmal malformation which classified mural type and underwent endovascular embolization of feeding arteries for vein of Galen aneurysmal malformation. After embolization, a vein of Galen aneurysmal malformation was immediately disappeared and tonsilar herniation improved in magnetic resonance imaging after 1 year embolized vascular malformation.

Discussion: This is a first case of reversible tonsilar herniation associated with vein of Galen malformation after endovascular embolization following changes over time. The mechanism of tonsilar herniation associated with vascular malformations are considered a mass of vascular malformations in posterior fossa and intracranial hypertension caused by arteriovenous shunt of vascular malformations.

168

Management and outcome of Moyamoya disease: A single institutional series of 81 patients

Yasuko Kusaka, Hideki Arakawa, Toshihiro Ishibashi, Yuichi Murayama Department of Neurosurgery, The Jikei University School of Medicine, Tokyo, Japan

Introduction: The efficacy of revascularization for moyamoya disease is believed to have therapeutic benefit for cerebral ischemic attacks. However, management strategy for asymptomatic and hemorrhagic patients are not well established. We report our therapeutic strategy for mayamoya and myamoya-like patients.

Methods: Between 2004 and 2013, 81 patients were referred. Initial onsets were ischemic (62 patients), hemorrhagic (6), and asymptomatic (13). There were 59 moyamoya cases including 12 children and 47 adults. Others were 9 adults with unilateral moyamoya and 13 moyamoya-like patients. Each patient was diagnosed with MRA, 3D-CT angiography and SPECT w/wo Diamox. We named cases as "moyamoya-like disease", which didn't meet diagnostic criteria of moyamoya disease. Single surgeon with same strategy performed surgical and conservative treatment. Results: All children received bypass surgery and revealed good outcome with WAIS-R test. We underwent bypass surgery for adults; 27 with ischemic onset, 2 hemorrhagic onset and 2 others. Morbidity rate was $9.7 \%$ with hemorrhage. 4 cases of 9 unilateral moyamoya and 6 cases of 13 moyamoya-like patients with bypass surgery had good outcome. All asymptomatic patients were treated conservatively and there was no cerebrovascular event during observation. 
Conclusions: Bypass surgery for ischemic patients was effective as ever known. For hemorrhagic onset and/or asymptomatic cases, we should perform tailor-made management with clinical and radiological investigations.

\section{9}

\section{Ballistic trauma in Pediatrics Population}

Dr Hassan Kadri

Neurosurgical Clinic, Medical and Scientific Research Center, damascus, Syria

Independent sources reported that more than 4000 kids were killed while 13000 were injured between 2011 and early 2013 in Syria. 42 patients aged between 2 months and 16 years were seen in critical conditions suffering from brain injuries by gunshot. 11 cases the bullet was present in the skull space (no exit site) while in 31 the bullet left the head. All those patients were managed in primary battlefield hospital set up in secrets by some doctors. The aim was: stopping the bleeding by local homeostasis, decompression the brain and in few cases removing the projectile. Glasgow scale score was evaluated for all patients it varied between 4 and 13 . 18 patients who arrived alive to medical unit, had an initial GS at 4 they died shortly after admission while 6 kids had GSS $<11$ needed artificial ventilation died later on because of lake or maladapted artificial ventilation. 18 kids underwent a surgical repair for their lesions. In those 18 patients who were evaluated as (in good condition), 10 of them had an outlet hole no XR was performed for them. While 8 out of the have no outlet hole, they had either an XR or CT scan in peripheral hospital. Transportation was itself an issue. The surgical repair varies between skin repair to prevent CSF leak, decompression by removal of bone fragments which were compressing the brain and local homeostasis. 8 patients died later from other complications a 10 stayed alive 3 of them have severe handicaps.

Conclusion: Unfortunately the penetrating head injury by a gunshot have a bad prognosisi when it is managed in poorly equipped medical unit.

\section{Nurse Symposium}

1

Nursing practical issues and concepts: Vagus nerve stimulation for epilepsy in children

Thresia Alex, Esther Kho-Uy

Montefiore Medical Center, Bronx, New York USA

Vagal Nerve Stimulation (VNS) is an alternative treatment for children whose seizures are not well controlled with medications and who are not candidates for an open brain operation to eliminate seizures. It can also be helpful for patients who have trouble following a medication routine or have severe side effects from multiple medications. With this VNS therapy few patients find that their seizures completely go away with VNS, but many are able to reduce their medications, giving them greater flexibility and confidence. This presentation will outline the surgical techniques, safety and efficacy, and theories on the mechanism of action of VNS in children with epilepsy.

Presentation of Dysembryoplastic Neuroepithelial tTmours (DNET), outcomes following epilepsy surgery and the supporting role the nurse practitioner may have in improving outcomes for the family. Nicola J. Barnes

Nurse Practitioner, Complex Epilepsy, Great Ormond Street Hospital, London, United Kingdom
DNETS. A benign low-grade lesion usually present with drug resistant epilepsy with focal seizure types originating from the temporal lobe. Temporal resection may look to offer positive outcomes against the continued use of medical management. This study will aim to complete systematic review of cases completed at Great Ormond Street Hospital over the last 3 years by pathology confirmation. It will look to define presentation, seizure types, age of seizure onset, duration of epilepsy prior to surgery, level of cognitive function, verbal and visual memory. Following surgery it will aim to identify epilepsy surgery outcomes, seizure freedom, possible reduction in anti-epileptic medication, decline or advancement in cognitive function and effect on QOL. It will acknowledge the families experience of the epilepsy surgery journey and how the role of the nurse practitioner support aims to enhance their understanding and acceptance of postoperative outcomes, by continuing accessibility of service to provide timely interventions if required such as AED reduction or even simple support increasing family's confidence in caring for their young person, for example promotion of more independence in a teenager that may now be seizure free. Initial review of data held noted 11 cases of confirmed DNET from 150 completed cases of epilepsy surgery, aged 4 months to 17 years, mean age of children undergoing surgery was 7.9 years and included 8 girls and 3 boys.

3

Endoscopic strip craniectomy: Minimally invasive correction for craniosynostosis

Cathy Cartwright, Usiakimi Igbaseimokumo

Section of Neurosurgery, Division of Surgery Children's Mercy Hospital Kansas City, Missouri USA

Introduction: Craniosynostosis occurs in 1:2100 children and can cause severe deformities to the face and skull. Extensive calvarial vault remodeling for correction can have lengthy operating times, extended postoperative hospital stays and prolonged recovery. When done before 5 months of age, the endoscopic strip craniectomy with postoperative molding helmet provides parents with a mininally invasive option for correction of craniosynostosis.

Methods: 46 patients from 2010-2013 underwent endoscopic strip craniectomy and postoperative helmet therapy. Thirty-six had sagittal synostosis, 3 metopic, 4 unilateral coronal, 1 bicoronal, 1 lambdoid and 1 multisuture (coronal/sagittal). There were 12 females and 34 males whose ages ranged from 3 weeks to 5.5 months. Length of stay ranged from 1 day ( 22 patients or 48\%), 2 days (19 patients or $41 \%$ ), 3 days (4 patients or $9 \%$ ) and 4 days ( 1 patient at $2 \%$ ).

Results: Each child showed improvement in appearance as evidenced in preoperative and postoperative comparison photographs. No child has had reoperation. No parent expressed regret for choice of surgical correction.

Conclusion: The endoscopic strip craniectomy with postoperative helmet therapy is a safe and effective option for infants less than 5 months of age for correction of craniosynostosis.

4

Ward assistence for craniosynostosis (CS) around surgical treatment Luigi di Somma, Ignazio Campanella, Rosanna Amari, Giovanna di Cosmo, Aleandro Vazquez, Carmine Manzione, Laura Grazia Valentini, Simona di Carlo

Department of Neurosurgery, Fondazione IRCCS Istituto Neurologico "C. Besta", Milan, Italy

Introduction: Surgery for craniosynostosis is highly demanding both for families and for medical staff, due to the small age of patients, complexity of surgery and anesthetic support needed and emotional charge.

Case Series: Cranioplasty, that is reshaping and repositioning of cranial bone segments, was the surgical treatment performed in our Department; this type of correction was performed in 220 children, affected by different Cranisynostosis (130 Scaphocephaly, 40 Trigonocephaly, 30 Anterior 
Plagiocephaly and 20 Polisynostosys) from January 2001 to December 2012. Age ranged from 5 to 26 months (average 9 months). Since 2005, due to an anesthetic protocol, children are not admitted in the Intensive Care Unit after surgery, but returned directly to the Ward after a few hours staying in Recovery Room for immediate correction.

Needs Analysis: Preoperatively the preparation needed hematologic study for anesthetic evaluation, the request for blood transfusions (preparation of micro ultrafiltered pockets of packed red blood cells and plasma. After surgery the close control of water balance related to intravenous intake and to early resumption of breastfeeding, pain control and wound medication. All along the peri-operative period there was a need of family support. Conclusions: To reply to the needs documented, we prepared protocols for water balance, wound medications, blood tests and transfusions, Antibiotico Prophylaxis. They will be discussed in detail. Moreover psychological support was organized by the parental association Aicra to solve for the emotional needs of the families.

Pathways and treatment guidelines for pediatric neurosurgical patients in an interdisciplinary surgical ward

Kristina Düngen

Asklepios Children's Hospital, Sankt Augustin, Germany

Introduction: Pediatric neurosurgical patients are treated in an interdisciplinary surgical ward. The core disciplines are pediatric surgery, orthopedic surgery and neurosurgery. All nurses take over significant responsibility for all kind of patients during the pre- and postsurgical period. About half of the neurosurgical patients are at potential risk of increased intracranial pressure, whereas other patients may develop problems related to the CSF-system.

Contents: In order to maintain a high safety level and smooth management procedures in these specific patients, nurses and doctors developed treatment and management guidelines for typical neurosurgical patients. They will be presented and discussed. Essential items are: pre- and postoperative management (in general, related to specific diagnosis), EVD-management, pain management.

"Too Little, Too Late?" Is involving palliative care at end of life too late?

Angela Forbes, Amanda J Breedt

Division of Neurosurgery, Seattle Children's Hospital, Seattle, Washington

The idea that palliative care is simply for the terminal stages of illness is out of date. Neurosurgical patients often involve complex and high risk care that require multi-faceted decision making by multiple team members. The patient and their families are often faced with over-whelming decisions that can often be life changing. This advance decision making support demonstrates the need for routine utilization of the palliative care team for this complex population. We will review current utilization of the palliative care team by the neurosurgery care providers and consider future use of their services. A detail discussion will be had on current practices of palliative care at a large urban medical center. This discussion will be lead using an illustration of three case studies demonstrating variable use of the palliative care team and assessing the outcomes of the patient.

\section{7}

The long-term effects for craniopharyngioma patients and the provision of family-centred education and support

Chloe Gillen, Maria Hunt

Great Ormond Street Hospital for Children, NHS Foundation Trust, London Southbank University

Childhood craniopharyngiomas are histologically benign tumours originating from the remnants of rathke's pouch in the hypothalamic pituitary region. Symptoms exhibited can have a devastating effect on both the patient's quality of life and their life expectancy, with co morbidities including diabetes insipidus, visual defects, hypothalamic obesity, hypothyroidism and behavioural changes. The management of craniopharyngiomas remains challenging and controversial. Although the histology of the tumour is benign, it is often clinically aggressive, with the patient likely to experience recurrences. The morbidity associated with craniopharyngionma at diagnosis and thereafter, can occur as a consequence of both neurosurgery and radiotherapy/ chemotherapy. It is advised that the treatment plan for craniopharyngioma patients must always involve the MDT, including the neurosurgeon, radiation oncologist, paediatric oncologist, endocrinologist and the nursing team, alongside the family. The literature suggests that nurses play a key part in the management, education and emotional support of children and families with craniopharyngioma. Nurses have a clear duty in educating these patients and their families about the full extent of the diagnosis and potential sequelae. Patients are more likely to comply with care requirements if they are educated well regarding the importance of rigorous long-term follow-up. A case study of a 16-year-old girl who was diagnosed with a craniopharyngioma at 8 -years-old will be used to illustrate the nurses role in the long term management, education and empowerment of these patients.

A review of symptoms described with adults with chiari malformation Lynda U. Gunn

Nurse Practitioner, Department of Neurosurgery, Sheffield Teaching Hospitals NHS Foundation Trust, United Kingdom

The clinical presentation of chiari malformation differs in adults, as compared to children. The most common presentation of chiari in an adult is the valsalva headache, though there is a great range of other symptoms many people also present with, including general headaches, visual disturbances, dysphagia, and a range of 'soft' neurological symptoms. Chiari malformation may lead on to developing syringomyelia, a cavitation within the spinal cord, which can lead to severe disability, muscle weakness, paralysis, and severe neuropathic pain and dysaesthesia. I will be discussing the most common presenting symptoms experienced by adults with chiari, and with adults presenting with syringomyelia. A case history will be included for demonstration purposes.

9

Is lumbar puncture a reliable measure of intracranial pressure? Usiakimi Igbaseimokumo, Cathy C. Cartwright, Christian B. Kaufman Section of Neurosurgery, Department of Surgery, Children's Mercy Hospital Kansas City, Missouri, USA

Introduction: Lumbar puncture to measure intracranial pressure (ICP) is limited by evaluating a single point in time. A microsensor placed in the brain is considered the gold standard because it is not affected by posture and it allows the child to mimic home activity over a longer period of time. This study investigates the reliability of lumbar puncture when compared to intracranial pressure monitoring with microsensor device for elective evaluation of suspected intracranial hypertension.

Methods: All patients over a three year period who had both lumbar puncture and ICP monitoring were reviewed. Demographic data, indications for monitoring ICP, symptoms, primary diagnosis, lumbar puncture and the intracranial pressure monitor pressures were collected and analyzed. Statistical analysis was with SPSS v 20 accepting a p-value of less than 0.05 as significant.

Results: There were 12 children ( 7 females: 5 males) aged 2 - 19 yrs (mean 11.1 years). The mean ICP measured by lumbar puncture was $22 \mathrm{mmHg}$ (range 18-26) and the mean ICP as measured with the microsensor (Camino bolt) was $7 \mathrm{mmHg}$ (range $3-12, \mathrm{p}<0.0001$ ). The primary 
diagnosis was craniosynostosis in 7 patients, hydrocephalus in 4 patients and pseudotumor in 1 patient. All patients had headache, one had papilledema. The anticipated surgical intervention was avoided in all the patients.

Conclusions: This study found a significant difference in the ICP measured by lumbar puncture and ICP monitor. We would recommend the use of ICP monitor in the evaluation of possible chronic raised intracranial pressure rather than a lumbar puncture.

10

Skin care during distraction osteogenesis for craniosynostosis Miku Kominami ${ }^{1}$, Miki Hatano ${ }^{1}$, Ayami Nakada ${ }^{1}$, Ayu Kasuga ${ }^{1}$, Satomi Hayashi $^{1}$, Chiemi Fukumoto ${ }^{1}$ Mihoko Kato ${ }^{2}$, Michihiro Kurimoto ${ }^{2}$, Goro Kondo $^{2}$, Hirokatsu Osawa ${ }^{2}$, Masato Nagasaka ${ }^{2}$

${ }^{1}$ Faculty of Nursing, ${ }^{2}$ Department of Neurosurgery, Aichi Children's Health and Medical Center, Aichi, Japan

Introduction: The distraction osteogenesis for Craniosynostosis(CS) causes many complications such as skin erosion, crust formation, ulceration and so on. This method requires careful skin care during hospitalization for distraction as well as consolidation period at home. Previously, we reported the effectiveness of coherent guidance using leaflets for parents. In this study, we tried to evaluate our refined skin care procedures during hospitalization.

Material and Methods: Thirty patients received cranioplasty from April, 2010 to March, 2013. We compared the outcomes of the patients with formerly reported 68 patients. We interviewed senior nursing stuffs about skin care and improved procedures were clarified.

Result: Refined care such as shampooing items or removing crust procedures were employed by senior nursing stuffs. The occurrence of skin complications showed less often in recent group than the former group. Discussion: We previously reported the importance of the coherent guidance for parents. Making parents participated in skin care after surgery succeeded in reducing skin complications. However, there were nearly $30 \%$ of complicated patients. We tried to reduce the complication rate by refining skin care during hospitalization. Although, the refine was minor, such as using shampooing items or removing crust more often, we could improve the results.

\section{1}

Late outcomes of the surgical management of anterior plagiocephaly: the predicting value of a classification based on the skull base anomalies Paola Leonardi

Pediatric Neurosurgery, Policlinic A. Gemelli, Rome, Italy

Introduction: Anterior plagiocephaly, though involving a single suture, is a challenging craniosynostosis, since the early closure of the hemicoronal suture also affects skull base and facial development. Despite effective surgical correction in infancy, the late aesthetic and functional results are still difficult to be predicted. Also it is difficult to identify those subjects who will require additional surgery late in life.

Methods: Patients operated on for anterior plagiocephaly in the pediatric neurosurgery unit of Policlinico Gemelli, Rome, between January 1980 and December 1989, who had undergone preoperative CT scan, were considered for the study. They were subgrouped in three types of increasing severity according to the classification proposed by the senior author in 1988. A computerized CT analysis was performed with the aim to evaluate the late outcomes and need of further maxillo-facial correction. The aesthetic self-perception of the patient was also taken into account. Results: Type I and IIA did not require additional surgical procedure and patients had generally no complaints about their cosmetic appearance. Type IIb and III required additional surgery (facial skeleton and orbital) in almost two thirds of cases as the facial asymmetry was associated to functional and emotional disturbance. Cognition was not affected independently from the degree of the malformation.
Conclusions: Skull base classification based upon CT scan evaluation is a reliable tool to predict the outcome of patients operated for anterior plagiocephaly. Based on it, nurses may provide to the parents the information about the long-term clinical and surgical pathway of the child.

12

Paediatric Neuroscience Benchmarking

Lindy May

Hospital for Children, Great Ormond St, London

Paediatric neuroscience benchmarking provides a framework by which to facilitate continuous improvement and promote evidence based practice through comparison and sharing across units. This is achieved by collaborative agreement, professional consensus, research and evidence based practice, comparison and sharing, avoid "reinventing the wheel", and monitoring practice development regularly. Networking is also promoted. The aim of the session is to discuss how benchmarking can be implemented within local units and will be followed by a benchmarking workshop.

13

The management of Chiari 1 in children

Lindy May

Great Ormond Street Hospital for Children, London, United Kingdom

Chiari 1 (CIM) and FMD there are four variants of Chiari malformation, with additional types 0 and 1.5 proposed on the basis of case reports. Chiari 1 (CIM) is defined as an isolated caudal displacement of the cerebellar tonsils more than $5 \mathrm{~mm}$ below the foramen magnum; medullary distortion may co-exist due to 'crowding' within the foramen magnum. The symptoms of CIM arise due to brainstem and spinal cord compression, or interruption of CSF flow, with a significant number of patients having syringomyelia. Lower cranial nerve signs including sleep apnoea, dysphagia and dysarthria, and drop attacks; ocular and vestibular symptoms may also occur and sensory changes in upper limbs and trunk. The clinical presentation in children may differ from adults, with headache, scoliosis and sleep apnoea being the most common presenting symptom in children. The International Headache Society (IHS) describes headaches as medically refractory headaches that are occipital, exacerbated by coughing, straining or the Valsalva manoeuvre. Chiari 1 is also considered as a possible cause of scoliosis in children, with an FMD providing adequate treatment for those with minor scoliosis, and FMD and spinal fusion required in severe cases. Co morbidities in children include Pfeiffer syndrome, Crouzon syndrome, Morquio syndrome and osteopetrosis. A case history of a 14 month old boy is provided to illustrate specific symptomology in children.

\section{4}

Nursing perspective of extra ventricular drain use on the pediatric neurosciences and surgery ward

Stephanie Obara

3R Neurosciences and Surgery, BC Children's Hospital, Vancouver, Canada

Extra Ventricular Drains (EVDs) are primarily used to allow the temporary drainage of cerebrospinal fluid (CSF) from the ventricles of the brain, preventing or relieving raised intracranial pressure. An EVD is also used to divert infected CSF, divert bloodstained CSF following neurosurgery/ hemorrhage, or divert the flow of CSF. The use of EVDs can be especially challenging in children due to varying factors such as age and level of neurological compromise. At our hospital, Registered Nurses are responsible for leveling, zeroing, setting, and maintaining an ordered point of drainage for an EVD system. The control of intracranial pressure is only done by Registered Nurses in the Pediatric Critical Care Unit. We will 
give a basic review of the EVD system used on our Neurosciences and Surgery ward, including related nursing education, policies and care plans. We will explore safety events encountered over the past year and will share some of the challenges from a nursing perspective. Our objective is to stimulate discussion and gather ideas regarding best practice for care of patients with EVDs.

15

Nursing care in the newborn whit Spina Bifida and hydrocephalus Marisol Rico Reyes ${ }^{1}$, Oscar Garcia Gonzalez ${ }^{2}$

${ }^{1}$ Facultad de Estudios Superiores Zaragoza, Universidad Nacional Autonoma de Mexico, León, Mexico; ${ }^{2}$ Pediatric Neurosurgery, Hospital Regional de Alta Especialidad del Bajio, León, Mexico

Introduction: The care of an infant with Myelomenigocele and hydrocephalus is managed using a multidisciplinary approach co-ordinated by the neonatology and spinal rehabilitation teams and including neurosurgery, urology, orthopaedics, physiotherapy and social work, the role of pediatric nurse is reviewed.

Material and methods: We review the current literature available related to the nursing care plan for the management of the children born whit open spinal defects and those who developed hydrocephalus. Presenting our own nurse care and the Institutional experience in the period between June 2009 - March 2013.

Results: The review or search about the Nursing Care Plans in Spina Bifida and hydrocephalus the access to the information is limited, there are a few publications without at least the abstracts available. We present our experience whit 20 newborns whit myelomeningocele at the Hospital Regionalde Alta Especialidad del Bajio in León, Mexico.

Conclusions: The Pediatric Nurse have the dual responsability in caring for a patient whit special medical needs, and the second is to care for the emotional needs of the family who have a newborn whit myelomeningocele. Based in the universal information that is available we have modified the nursing plans to our own institutional, economical and social conditions.

\section{6}

Treatment of intraventricular arachnoid cysts: Role of nurse in assistance of endoscopy and shunts in infants

Anna Rossi, Monica Viotto, Renata Maglieri, Vincenzo Andriulo, Laura Valentini, Marika Furlanetto

Fondazione Istituto Nazionale Neurologico "Carlo Besta" (FINCB), Milano, Italy

Introduction: The prognosis of intraventricular cyst has been reported to be favourable both in neonatal and in pediatric age, except in case of a posthemorrhagic or infective ones. Arachnoid cysts have a better prognosis, despite their progression is still unpredictable. One of the principal problems related to fetal and neonatal CSF flow disturbances is due to the risk of inducing postshuntig synostosis.

Patients and Clinical Picture: The present study concerns 15 children treated by endoscopy or shunting at the National Neurological Institute (FINCB) for intraventricular cysts due to dilatation of arachnoid cysts occurred in the late pregnancy or first 3 months after birth. Indication for surgery was occurrence of symptoms and/or increase of the ventricles or cysts. Twelve patients were fenestrated as the first choice, six were treated by first choice with programmable shunting, three after fenestration failure. Afterwards all the children have been followed for at least 1 year $(\min 18 \max 60$ months) postoperatively and they were submitted to clinical evaluation and RM at Follow-Up.

Results: There was no mortality. No post-shunting craniosynostosis was observed. There were 2 reoperation for shunt malfunction. The major problem documented in endoscopy was CSF leak/collection. The outcome was favourable in all the cases except two, without correlation with the causes of CSF circulation problems, but with its cause (hemorrhage and infection). All the children shunted needed at least 2 or more pressure adjustment during the first 18 months, while in the endoscopic treated the cysts progressively reduced along the follow-up.

Conclusions: The analysis of the present series suggests that precocious treatment of CSF circulation disturbances could be correlated with an early parenchyma re-expansion and favourable neurological outcome. Three failures were observed in younger age; in the neonates if shunting procedure is performed; the fenestration may be postponed, at time of shunt malfunction. Minivasive technique by endoscopic fenestration is suitable for intraventricular cyst, but it needs dedicated small instrumentation to prevent CSF leak; an accurate assistance by nurses is required for the set-up, use and maintenance of this delicate and fragile tool to obtain and preserve good vision.

\section{7}

Interactive neurosciences benchmarking session

Jenny Sacree

Frenchay Hospital, Bristol, United Kingdom

Clinical practice benchmarking is a quality improvement tool. It facilitates structures and formalises how best practice is compared, shared and developed. It inspires nurses to make changes in practice and reassures everyone they are doing the best they can to develop and improve quality of care (Ellis J 2007). Within the British Isles there is an active neurosciences benchmarking group, sharing and developing best practice within our own units. I would like to share a typical benchmarking exercise (interactive session) around some simple subjects that we all experience within our daily workload. Allowing us to share some best practice and new ideas, and so leading us all to a better understanding and enhancing our international working relationships.

\section{8}

Tools available to advanced nurse practitioners to diagnose shunt malfunctions in a child with unchanging neuro-radiology - a critical analysis of the published tools

Jenny Sacree

Frenchay Hospital, Bristol, United Kingdom

Hydrocephalus and its treatment represent a large proportion of a paediatric neurosurgeons workload, ventricular-peritoneal shunting remaining the mainstay of treatment, bringing with it, its own raft of issues with malfunctions. The most common tools used in the diagnosis of a shunt malfunction, are a good clinical history and a head CT scan. For the majority of patients, presenting with a possible shunt malfunction, this neuro-imaging will show an increased ventricular size, compared to a previously imaging, indicating a rise in intraventricular/intracranial pressure. Further intervention is not required and the patient can proceed to a shunt revision. There are however a portion of patients who will present with much more subtle signs and symptoms, with unchanging neuroradiology and imaging. These patients present a significant challenge of diagnosis to the neurosurgeon leading their care, leaving them faced with the difficult decision between operative investigation, with its associated risks of infection or conservative treatment and possible subsequent very rapid deterioration. In the $21^{\text {st }}$ century the role of the advanced practice nurse is expanding and developing to meet the challenges that face every hospital and trust, with a much more active and frontline role taken on by advanced practitioners. It is, therefore, ever more important to have robust evidence based guidelines and pathways for these practitioners to follow. This paper presents a comprehensive literature review and critical analysis of published tools available for the advanced practitioner to use to 
diagnose a shunt malfunction in the patient with unchanging radiological findings.

\section{9}

\section{The 3 C's of neuroscience nursing}

Satiapoorany Subramaniam

National University of Malaysia, Medical Centre, Kuala Lumpur, Malaysia

Nursing is a demanding yet rewarding profession. Neurosurgical Unit is a unit where nurses embrace a holistic, coordinated, multidisciplinary approach to meeting patients and family's needs and expectations, thereby ensuring patient safety and patient and staff satisfaction. Therefore nurses play a vital role in facilitating a shift towards a more patient-care focused environment. The $3 \mathrm{C}$ 's of neuroscience nursing of CARING, COMPASSION AND COMMUNICATION is very vital in neuroscience nursing. The mission of every neuroscience nurse is to be a leader in providing compassionate, individualized, culturally appropriate quality nursing care, focusing on the unique needs of our neurosurgical patient population and their families. Communication has always been the cornerstone of nursing care, so much so that it is almost impossible to describe what nursing is or what nurses do without reference to terms such as listening, communicating, reporting and observing. However, compassion is also linked along with confidence, competence, conscience, commitment and comportment. If nurses claim to genuinely care for their patients, then without compassion their caring may be incomplete and lacking. This could help to explain why some nurses are technically competent, but do not seem outwardly compassionate. Ultimately, "compassion impels and empowers nurses to not only acknowledge, but also act". The shift towards patient-centered care is the priority in health care today. Providing quality care with the $3 \mathrm{C}^{\prime}$ that is highly efficient and patient-friendly while being cost-effective is a difficult, but not impossible task.

\section{0}

The use of play therapy in MRI preparation for children

Yoshimi Tanase $^{1}$, Teruko Banno ${ }^{1}$, Yuko Hirano ${ }^{1}$, Syoko Omori ${ }^{1}$, Yuki Miyatani ${ }^{1}$, Mihoko Kato ${ }^{2}$, Hirokatsu Osawa ${ }^{2}$, Midori Yanase ${ }^{3}$, Yasuhiro Iwasaki ${ }^{3}$, Masato Nagasaka ${ }^{2}$

${ }^{1}$ Child care service, ${ }^{2}$ Department of Neurosurgery, ${ }^{3}$ Department of Radiology, Aichi Children's Health and Medical Center, Nagoya, Japan

Magnetic resonace imaging (MRI) plays an important role on the evaluation of pediatric physical conditions such as brain. However, small children tend to be intimidated by the environment and need sedation because of long scanning time, narrow tunnel, and noise. Recently, play therapy in preparation of MRI for young kids has been considered to be crucial to alleviate fear and thus reduce the necessity of sedation. In our facility, five hospital play specialists as staff have been joining MRI play preparation (PP) using a puppet, "Ray-chan". The purpose of the study is to evaluate the efficacy using our PP procedure for MRI. We conducted non-sedation PP for 94 patients in our facility between July 2010 and March 2013. The age of the patients was from 4 to 15 years and 76 children (80\%) achieved MRI successfully after PP. Prior to non-sedation PP, we have already started PP with sedation since 2009 and 23 of 28 (79\%) kids successfully finished MRI. As the success rate of MRI without sedation was almost similar to those with sedation followed by $\mathrm{PP}$, it suggested that our protocol of PP had potential to decrease the usage of sedative drugs. In the presentation, we will exhibit our methods of PP using sounds, songs, fairy tale, picture books, and puppet. Moreover, we plan to mention our effort such as improving our technique and proper choice of words \& tools to make more comfortable situation for kids, especially patients with developmental delay and prevent psychological trauma from MRI.

\section{1}

A retrospective review of External Ventricular Drain surveillance and management by the MDT

Tracey Waterston, Kathryn Sethi

Birmingham Children's Hospital

External ventricular drain (EVD) catheters are frequently used in the paediatric neurosurgery setting; however they are not without complications which can at times be life threatening. This study examinations the causes, reporting processes, and treatment of EVD under drainage and blockage on PICU, NSW and the neurosurgical ward.

This study was undertaken to review the established practices, and identify any areas of variance between specialist care areas. To highlight and implement improvements in practice to deliver consistent, quality patient care.

Methods: a retrospective case review of all patients requiring EVD insertion between January - June 2013, on PICU, Neonatal Surgery Ward (NSW) and the neurosurgical ward. Primary outcomes will identify:

1. Factors that cause under drainage/blockage.

2. Time under drainage/blockage first reported to medical staff

3. Treatment response time (number of hours of reduced/no drainage)

4. Treatments and outcomes of under drainage/blockage.

Secondary Outcomes will identify:

1. The factors that may delay the treatment response times by nursing/ medical team

2. Current variance in practices between care areas

3. Factors that cause variance in treatments between care areas.

The findings will identify areas of variance in treatment and inform the revision of current EVD protocols and guidelines ensuring a consistent approach to care of the child with an EVD is undertaken across the specialties.

\section{2}

Nursing the craniopharyngioma child $-\mathbf{A}$ case presentation

Yee Yit Cheng

Nursing Manager, Department of Neurosurgery, Kuala Lumpur General Hospital, Malaysia

Craniopharyngiomas are brain tumours that originate in the anterior superior margin of the pituitary gland. Pituitary gland involvement causes loss of pituitary function. Symptoms of intracranial mass effect including visual field loss, obstructive hydrocephalus and extracular palsy of the cranial nerves may occur. Lifelong follow-up is crucial because of recurrence and the need for ongoing hormone replacement therapy. Therefore nurses play an important role in caring for patients with craniopharyngioma by preventing and identifying complications, educating family and make family understand the importance of long-term follow-up, treatment required and side effects of medications in order to prevent morbility and promote recovery. 